\title{
Surface-Water Quantity and Quality Data, Rocky Flats Environmental Technology Site Near Denver, Colorado, Water Years 1994-95
}

By Mark E. Smith, John W. Unruh, and Clayton H. Thompson

\section{U.S. GEOLOGICAL SURVEY}

Open-File Report 96-314

Interim Report

Prepared in cooperation with the U.S. Department of Energy

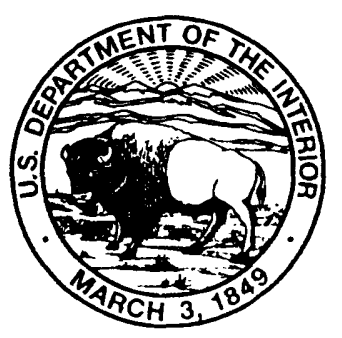




\title{
U.S. DEPARTMENT OF THE INTERIOR BRUCE BABBITT, Secretary
}

\author{
U.S. GEOLOGICAL SURVEY
}

Gordon P. Eaton, Director

The use of firm, trade, and brand names in this report is for identification purposes only and does not constitute endorsement by the U.S. Geological Survey

For addtional information write to:

District Chief

U.S. Geological Survey

Box 25046, Mail Stop 415

Denver Federal Center

Denver, CO 80225-0046
Copies of this report can be purchased from:

U.S. Geological Survey

Information Services

Box 25286, Mail Stop 306

Denver Federal Center

Denver, CO 80225-0046 


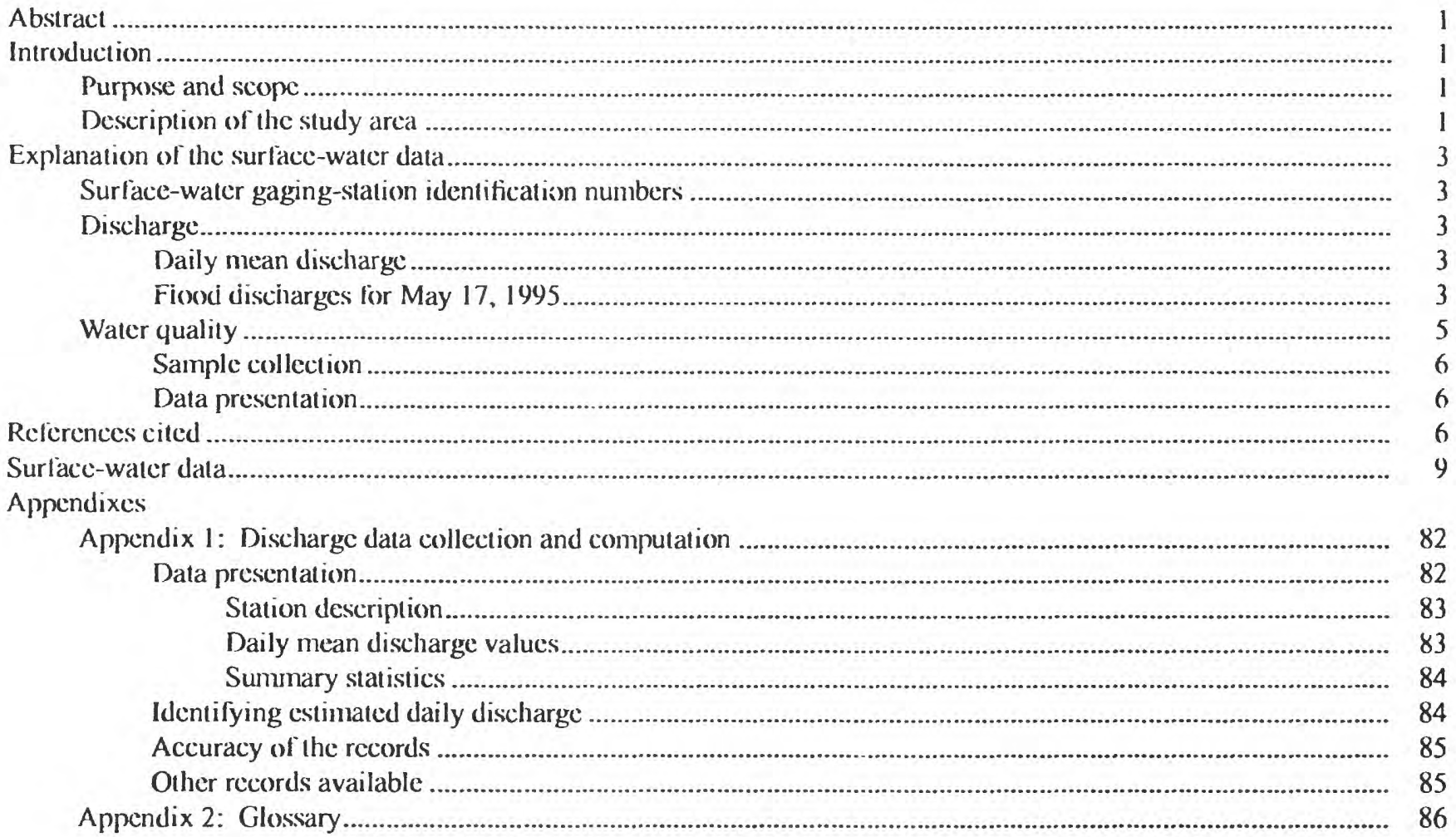

\section{FIGURE}

1. Map showing location of surface-water gaging stations and selected surface-water features at the

Rocky Flats Environmental Technology Site, water years 1994-95

\section{TABLES}

1. Surface-water gaging stations at the Rocky Flats Environmental Technology Site

2. Peak and daily mean discharges for Woman Creek, flood of May 17, 1995

3. Analytes for samples collected during water years 1994-95.

4 40. Daily mean discharge:

4. GS01 (Woman Creek at Indiana Street), water year 1994 ......................................................................... 11

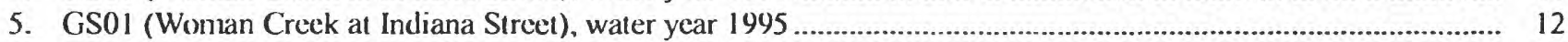

6. GS02 (Mower Ditch at Indiana Street), water year 1994 ........................................................................ 13

7. GS02 (Mower Ditch at Indiana Street), water year 1995 ..................................................................... 14

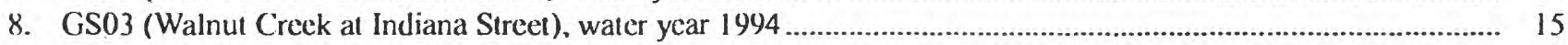

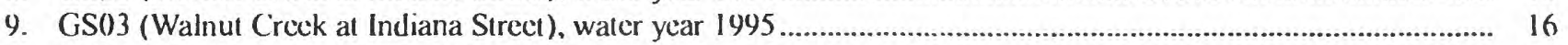

10. GS05 (North Woman Creek at West Buffer Zone Fence Line), water year 1994 .............................................. 17

11. GS05 (North Woman Creek at West Buffer Zone Fence Line), water year 1995 ............................................... 18

12. GS06 (South Woman Creek at West Buffer Zone Fence Line), water year 1994 ............................................... 19

13. GS06 (South Woman Creek at West Buffer Zone Fence Linc), water year 1995 ................................................. 20.

14. GS09 (South Walnut Creek Below Pond B-4), water year 1994 ................................................................... 21

15. GS09 (South Walnut Creek Below Pond B-4), water year 1995 ...................................................................... 22

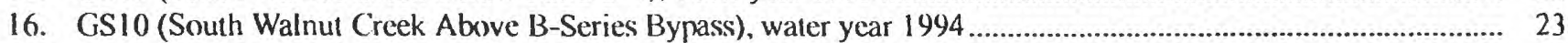

17. GS10 (South Walnut Creek Above B-Series Bypass), water year 1995 ........................................................ 24 


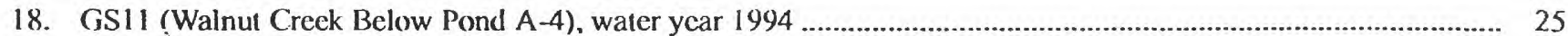

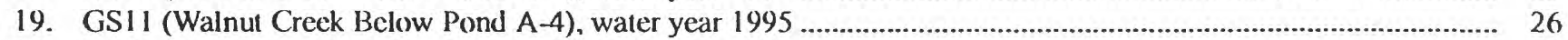

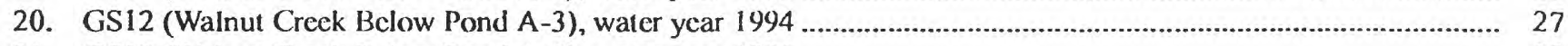

21. GS12 (Walnut Creek Below Pond A-3), water ycar 1995 .......................................................................... 28

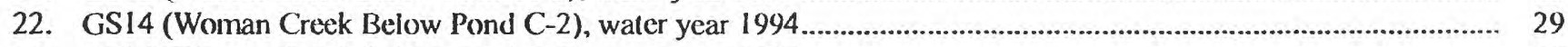

23. GS14 (Woman Creek Below Pond C-2), water year 1995 ............................................................................ 30

24. GS15 (Smart Ditch Above Pond D-1), water year 1994 ........................................................................... 31

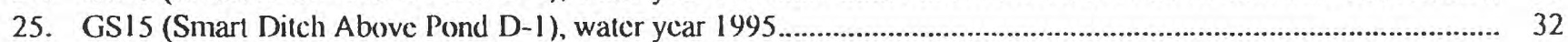

26. GS16 (Antelope Springs Above Woman Creck), water year 1994 ................................................................... 33

27. GS16 (Antelope Springs Above Woman Creck), water year 1995 ............................................................ 34

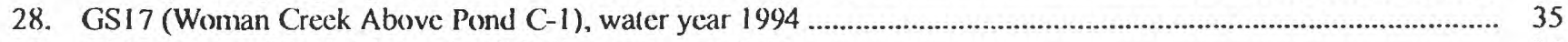

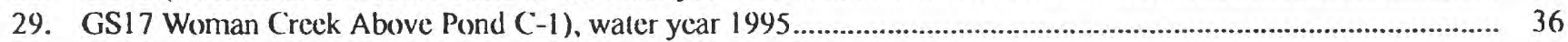

30. GS18 (Woman Creek Above Old Landfill), water year 1994 ................................................................ 37

31. GSI 8 (Woman Creck Above Old Landfill), water year 1995 .................................................................... 38

32. SW027 (South Interceptor Ditch Above Pond C-2), water year 1995 ............................................................ 39.

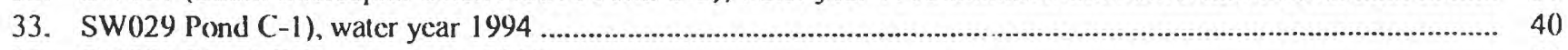

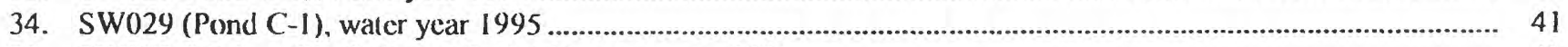

35. SW093 (Walnut Creck Below Portal 3), water year 1994 ............................................................................. 42

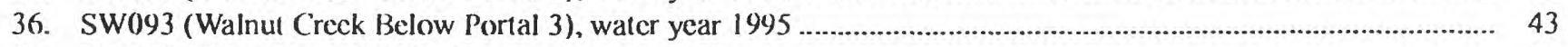

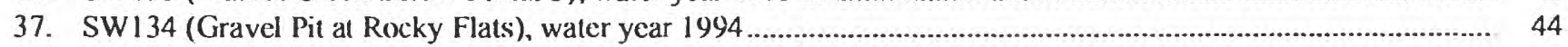

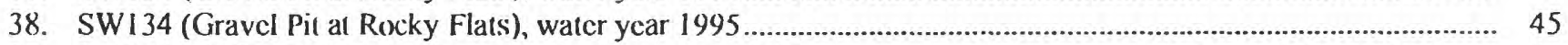

39. SW998 (T-130 Dilch at McKay Bypass), water year 1994 ....................................................................... 46

40. SW998 (T-130 Ditch at McKay Bypass), water year 1995 ......................................................................... 47

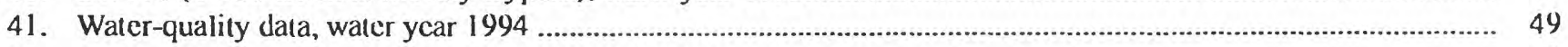

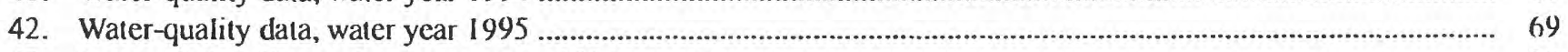

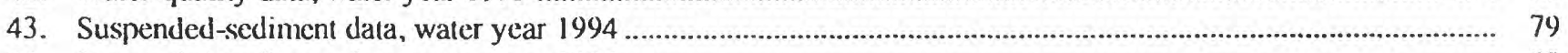

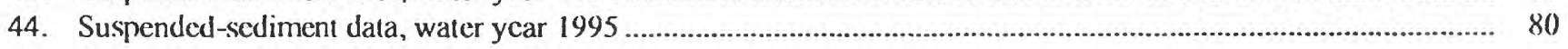

\section{CONVERSION FACTORS AND VERTICAL DATUM}

\begin{tabular}{|c|c|c|}
\hline Multiply & By & To obtain \\
\hline acre & 0.404687 & hectare \\
\hline acre-foot (acre-ft) & 1233.5 & cubic meter \\
\hline cubic foot $\left(\mathrm{tt}^{3}\right)$ & 0.028317 & cubic meter \\
\hline cubic foot per second $\left(\mathrm{ft}^{3} / \mathrm{s}\right)$ & 0.02832 & cubic meter per second \\
\hline foot (it) & 0.3048 & meter \\
\hline gallon (gal) & 0.0037854 & cubic meter \\
\hline gallon per minute (gal/min) & 3.7848 & liter per minute \\
\hline gallon per second (gal/s) & 3.7848 & liter per second \\
\hline inch & 25.4 & millimeter $(\mathrm{mm})$ \\
\hline mile (mi) & 1.609 & kilometer \\
\hline square mile $\left(\mathrm{mi}^{2}\right)$ & 2.590 & square kilometer \\
\hline
\end{tabular}

Degrec Celsius $\left({ }^{\circ} \mathrm{C}\right)$ may be converted to degrec Fahrenheit $\left({ }^{\circ} \mathrm{F}\right)$ by using the following equation:

$$
{ }^{\circ} \mathrm{F}=9 / 5\left({ }^{\circ} \mathrm{C}\right)+32
$$

Degrec Fahrenheit $\left({ }^{\circ} \mathrm{F}\right)$ may be converted to degrec Celsius $\left({ }^{\circ} \mathrm{C}\right)$ by using the following equation:

$$
{ }^{\circ} \mathrm{C}=5 / 9\left({ }^{\circ} \mathrm{F}-32\right)
$$

Sea level: In this report "sea level" refers to the National Geodetic Vertical Datum of 1929 (NGVD of 1929)- a geodetic datum derived from a general adjustment of the first-order level nets of both the United States and Canada, formerly called Sea Level Datum of 1929. 


\title{
Surface-Water Quantity and Quality Data, Rocky Flats Environmental Technology Site Near Denver, Colorado, Water Years 1994-95
}

\author{
By Mark E. Smith, John W. Unruh, and Clayton H. Thompson
}

\section{ABSTRACT}

Collection of surface-water quantity and quality data at the Rocky Flats Environmental Technology Site is needed as part of the ongoing cnvironmental clcanup of the facility, which is owned by the U.S. Department of Energy and operated by a private contractor. In May 1993, the U.S. Department of Energy entered into an interagency agrecment with the U.S. Geological Survey to collect surface-water quantity and quality (chemical and suspended-sediment) data at the Rocky Flats Environmental Technology Site. This report presents data collected by the U.S. Geological Survcy at 19 surfacc-water gaging stations during water years 1994-95. Quantity data, consisting of daily mean discharges. were collected and computed by the U.S. Gcological Survey. Chemical-quality data were collected by the U.S. Geological Survey, then were analyzed independently by the operating contractor; analytical results presented in this report were provided to the U.S. Geological Survey by the operating contractor. Sediment data (suspended-sediment concentrations and sand breaks) were collected and analyzed by the U.S. Geological Survey.

\section{IINTRODUCTION}

Collection of surface-water quantity and quality data at the Rocky Flats Environmental Technology Sitc (hereinafter, the Site) is needed as part of the ongoing environmental clcanup of the facility, which is owned by the U.S. Department of Encrgy (DOE) and operated by a privatc contractor (EG\&G Rocky Flats, Incorporated through June 1995; Rocky Mountain Remediation Services, L.L.C. beginning July 1995). In May 1993, the DOE cntered into an interagency agrecment with the U.S. Geological Survey (USGS) to collect surface-water quantity and quality (chemical and suspended-sediment) data and to operate and maintain surface-water gaging stations at the Site. The USGS collected data at 19 surface-water gaging stations at the Site (fig. 1) during water ycars 1994-95.

Surfacc-water quantity data can bc used by the DOE and the operating contractor for making watermanagement decisions at the Sitc throughout the year. Surface-water quality data can be used by the DOE and the operating contractor to characterize and cvaluate the quality of water flowing across the Sitc.

\section{Purpose and Scope}

This report presents surface-water hydrologic data collected by the USGS at the Site during water years 1994-95. These data include daily mean discharge and the analytical results of water-quality and suspended-sediment samples collected at 19 surfacc-water gaging stations (fig. 1). This report provides summary tables of the data collected at the Site, but does not provide any interpretation of the data beyond a qualitative rating of record quality (good, fair, or poor).

\section{Description of the Study Area}

The Site is situated on about 6,550 acres in northern Jefferson County, abou: 16 mi northwest of Denver, Colorado. Industrial facilities occupy about 384 acres near the center of the Site, and the remaining arca serves as a buffer zone between these facilities and the Site boundary (EG\&G Rocky Flats, Incorporated, 1993a). The USGS hydrologic network is in this buffer-zone area of the Site (fig. 1). 


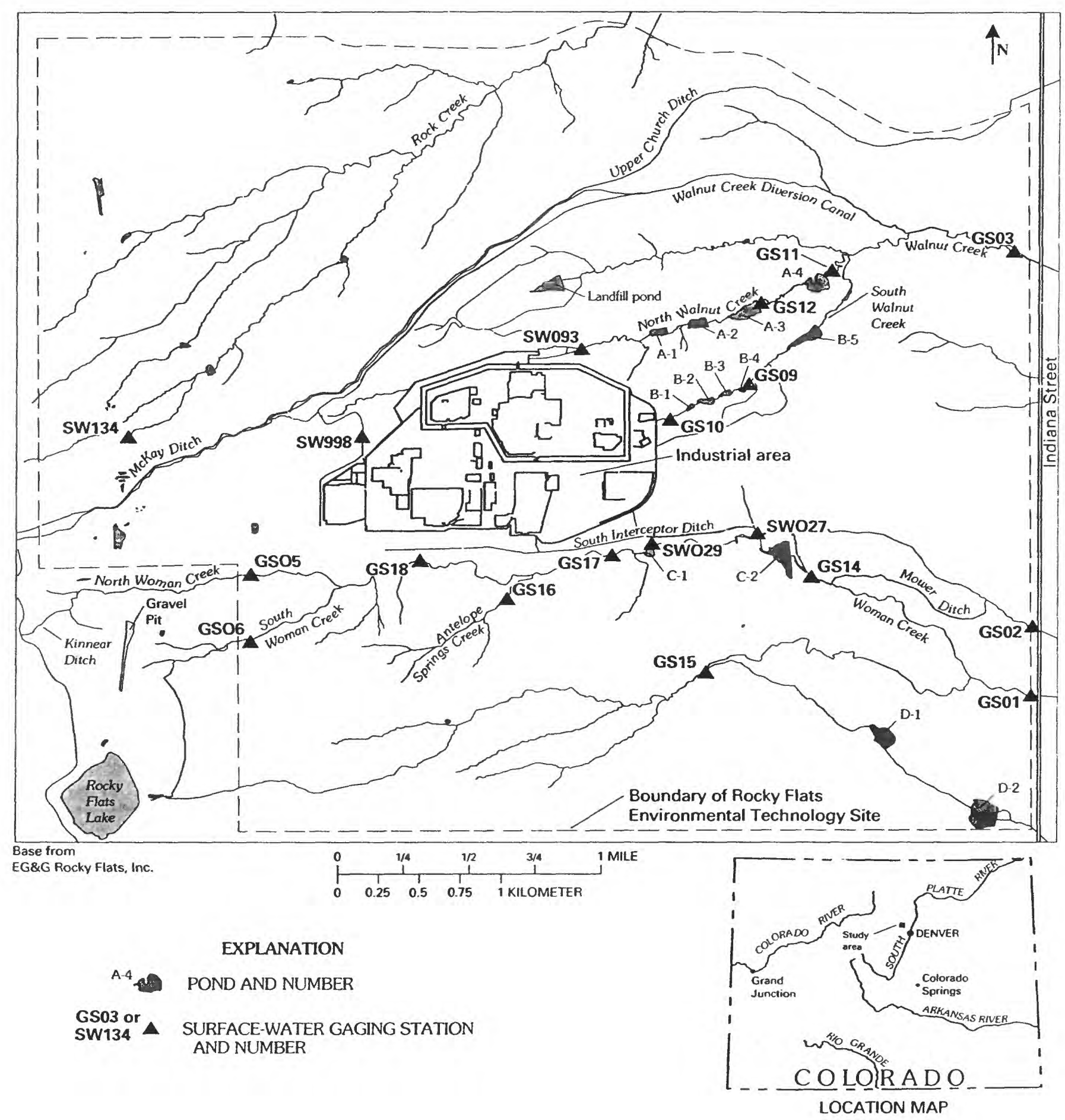

Figure 1. Location of surface-water gaging stations and selected surface-water features at the Rocky Flats Environmental Technology Site, water years $1994-95$. 
EXPLANATION OF THE SURFACE-WATER DATA

The surface-water quantity data published in this report consist of daily mean discharges fer water years 1994-95. Water year 1994 began October 1, 1993, and ended September 30, 1994. Daily mean discharge data reported by the USGS for water ycar 1994 bcgan in March, April, or May of 1994, depending on the activation date for each gaging station; carlier data for water ycar 1994 (Octuber and November 1993) are reported by Kocky Mountair Remediation Services, L.L.C. (1995) and are not published in this report. Water year 1995 began October 1, 1994, and ended September 30, 1995. All daily mean discharge data at the Site for water year 1995 were measured by the USGS and are published in this report. A detailed explanation of how the discharge data were collected, analyzed, computed, and arranged for presentation in this report is provided in Appendix 1 ("Discharge-Data Collection and Computation") at the back of this report. Definitions of hydrologic terms used in this report are provided in Appendix 2 ("Glossary") at the back of this report.

The surface-water quality data published in this report consist of analytical results for all samples collected at the Site by the USGS in water years 1994-95. Field water-quality measurements (water temperaturc, specific conductance, and $\mathrm{pH}$ ) and chemical-quality samples were collected by the USGS. Chemicalquality samples were analyzed independently by EG\&G Rocky Flats, Incorporated. Sediment samples (suspended-sediment concentrations and sand breaks) were collected and analyzed by the USGS.

\section{Surface-Water Gaging Station Identification Numbers}

The USGS assigned a 15-digit station identification number to cach gaging station identificd in this report. The station identification usually is the latitude and longitude of the gaging station with a sequence number $(00)$ at the end. Each gaging station in this report also was assigned a unique site number (for example, GS(1) by EG\&G Rocky Flats, Incorporated, as part of previous studics at the Site.

\section{Discharge}

The USGS gaging stations are designed to provide continuous records of discharge at each gage. Gaging stations opcrated at the Site by the USGS in water years 1994-95 arc listed in table 1 and their locations are shown in figure 1 . Continuous records of discharge are obtained using a continuous stagerecording device and a rating curve to convert observed stage values to discharge. Records of stage, recorded at 15-minute intervals, can be used to computc instantaneous discharge (at a given 15-minute intcrval) or daily mean discharge (mean for 24 hours). Daily mean discharges are presented in this report.

\section{Daily Mean Discharge}

Daily mean discharge data for cach gaging station are listed in tables $4-40$ in the "Surface-Water Data" scction; data are ordered by site number (GS01, SW027, and so forth) as listed in table 1. Much of the gaging-station descriptive information at the beginning of each table was provided by EG\&G Rocky Flats, Incorporated (1993b) and was reviewed by the USGS prior to publication in this report.

\section{Flood Discharges for May 17, 1995}

Most gaging stations at the Site were overtopped during the flooding on May 17, 1995. After flooding subsided, four gaging stations in the Woman Creek drainage were surveyed to compute peak (instantaneous) discharge by indirect methods (Benson and Dalrymple, 1967). Pcak discharges were computed for gaging stations GS05, GS06, GS 16, and SW029. Peak discharges at gaging stations GS05, GS06, and GS 16 were computed using the slope-area method (Dalrymple and Benson, 1967); peak discharge at gaging station SW029 was estimated using the culvert-rating method (Bodhaine, 1968). Daily mean discharges at gaging stations GS05, GS06, GS16, and SW029 were estimated using rating-curve extension to the peak discharges, available gageheight data, hydrographic comparison, and other observed flood information. One current-meter measurement of discharge was made at gaging station SW029 during the flood recession. 
Table 1. Surface-water gaging stations at the Rocky Flats Environmental Technology Site

[Locations of gaging stations are shown in figure 1; USGS. U.S. Geological Survey]

\begin{tabular}{|c|c|c|}
\hline $\begin{array}{l}\text { USGS station } \\
\text { identification }\end{array}$ & Site number & Gaging-station name \\
\hline 395240105095500 & GSO1 & Woman Creek at Indiana Street \\
\hline 395253105095506 & $\mathrm{GSO} 2$ & Mower Ditch at Indiana Street \\
\hline 395407105095900 & GS03 & Walnut Creek at Indiana Street \\
\hline 395306105131700 & GS05 & North Woman Creek at West Buffer Zone Fence Line \\
\hline 395253105131700 & GS06 & South Woman Creek at West Buffer Zone Fence Line \\
\hline 395342105110800 & GSOY & South Walnut Cireek below Pond B-4 \\
\hline 395335105112700 & GSio & South Walnut Creek above B-Series Bypass \\
\hline 395403105104700 & GSII & Walnul Creek below Pond $\Lambda-4$ \\
\hline 395358105110500 & GSI2 & Walnut Creek below Pond $\mathrm{A}-3$ \\
\hline 395304105105100 & GSI4 & Woman Creck below Pond C-2 \\
\hline 395246105111800 & GS15 & Smart Ditch above Pond D-1 \\
\hline 395301105120800 & GSI6 & Antelope Springs Creek above Woman Creek \\
\hline 395309105114100 & GSI7 & Woman Creek above Pond $\mathrm{C}-\mathrm{I}$ \\
\hline 395308105123100 & GS18 & Woman Creek above Old Landfill \\
\hline 395313105110500 & SW027 & South Interceplor Ditch above Pond C-2 \\
\hline 395310105113300 & SW029 & Pond C-1 \\
\hline 395349105114900 & SW093 & Walnut Creek below Portal 3 \\
\hline 395331105134400 & SWI34 & Gravel Pit at Rocky Flats \\
\hline 395332105124600 & SW998 & T-130 Ditch at McKay Bypass \\
\hline
\end{tabular}

Pcak and daily mean discharges for gaging station GS02 were computed from reliable gage-height data. Field conditions were not suitable for indirect measurement of peak discharge at gaging station GS01, but a daily mean discharge was estimated using visual observations during the flood and hydrographic comparison with other gaging stations. The flood-discharge data and associated information are listed in table 2.

Table 2. Peak and daily mean discharges for Woman Creek, flood of May 17, 1995

[f ${ }^{2} / \mathrm{s}$. cubic feeı per second: \pm . plus or minus: --.. dala unavailable]

\begin{tabular}{|c|c|c|c|}
\hline $\begin{array}{l}\text { Site } \\
\text { identification }\end{array}$ & $\begin{array}{l}\text { Peak } \\
\text { discharge } \\
\left(\mathrm{ft}^{3} / \mathrm{s}\right)\end{array}$ & $\begin{array}{l}\text { Daily mean } \\
\text { discharge } \\
\left(\mathrm{ft}^{3} / \mathrm{s}\right)\end{array}$ & Remarks \\
\hline GS05 & 238 & 21 & $\begin{array}{l}\text { Peak discharge by slope-area method (reliability: } \pm 25 \text { percent); daily mean } \\
\text { discharge estimated using various methods }\end{array}$ \\
\hline GS06 & 78 & 10 & $\begin{array}{l}\text { Peak discharge by slope-area method (reliability: } \pm 15 \text { percent); daily mean } \\
\text { discharge estimated using various methods } 1\end{array}$ \\
\hline SW029 & 380 & 93 & $\begin{array}{l}\text { Current-meter measurement of } 203 \mathrm{ft}^{3} / \mathrm{s} \text { on recession; peak and daily mean } \\
\text { discharge estimated by culvert-rating method (reliability: peak. } \pm 25 \text { percent; } \\
\text { daily mean, } \pm 15 \text { percent) }\end{array}$ \\
\hline GSO2 & 14 & 8.8 & Discharges computed from valid gage-height data \\
\hline GSOI & -- & 76 & $\begin{array}{l}\text { Daily mean discharge estimated using flood observations and hydrographic } \\
\text { comparison }\end{array}$ \\
\hline
\end{tabular}

'Estimation methods included ratıng-curve extension on the peak discharges, available gage-height data. hydrographic comparison. and other observed floced information.

4 Surface-Water Quantity and Quality Data, Rocky Flats Environmental Technology Site Near Denver, Colorado, Water Years 1994-95 
Indirect methods are described in gencral by Benson and Dalrymple (1967); the slope-area method by Dalrymple and Benson (1967); and the culvertrating method by Bodhaine (1968).

\section{Water Quality}

The USGS collected surface-water quality (chemicai and suspended-sediment) data in water years 1994-95. A list of the analytes sampled by the
USGS is in table 3. Water temperature, specific conductance, and $\mathrm{pH}$ were measured by the USGS during sample collection; mean discharge for each sample was computed by the USGS from instantaneous-discharge data. All other chemical-quality samples collected by the USGS were sent to EG\&G Rocky Flats, Incorporated for analysis. Analytical results for these samples were retrieved from the Site computer data base, maintained by EG\&G Rocky Flats, Incorporated, for publication in this report. Analytical results are presented as retricved from that data base, without modification by the USGS.

Table 3. Analytes for samples collected during water years $1994-95$

[ICPES. Inductively coupled plasma emission spectroscopy: GFAA. Graphile fumace atomic absomption: CVAA. Cold vapor atomic absorpluon: ICPMS. Inductively coupled plasma mass spectroscopy|

\begin{tabular}{|c|c|c|c|c|}
\hline \multicolumn{2}{|c|}{ Metals, total and dissolved } & \multirow{2}{*}{$\begin{array}{l}\text { Radionuclides, total } \\
\text { Plutonium } 239 / 240\end{array}$} & \multirow{2}{*}{$\begin{array}{l}\text { Water-quality } \\
\text { properties and } \\
\text { constituents }\end{array}$} & \multirow{2}{*}{$\begin{array}{l}\text { Suspended sediment } \\
\text { Concentration }\end{array}$} \\
\hline By ICPES & By GFAA & & & \\
\hline Aluminum & Arsenic & Americium 241 & Specific conductance & Sand-break fraction \\
\hline Antimony & Lead & Uranium 233, 234 & $\mathrm{pH}$ & \\
\hline Arsenic & Selenium & Uranium 238 & Alkalinity & \\
\hline Barium & Silver & Uranium 235 & Chloride & \\
\hline Beryllium & Cadmium & Tritium & Fluoride & \\
\hline Cadmium & & Gross alpha & Sulfate & \\
\hline Calcium & By CVAA & Gross beta & Nitrate/nitrite & \\
\hline Chromium & Mercury & & Total phosphorus & \\
\hline Cobalt & & & Total suspended solids & \\
\hline Copper & By ICPMS & & & \\
\hline Iron & Arsenic & & & \\
\hline Lead & Lead & & & \\
\hline Lithium & Selenium & & & \\
\hline Magnesium & Thallium & & & \\
\hline Manganese & Tin & & & \\
\hline Molybdenum & Cesiun & & & \\
\hline Nickel & Uranium (total) & & & \\
\hline \multicolumn{5}{|l|}{ Potassium } \\
\hline \multicolumn{5}{|l|}{ Sclenium } \\
\hline \multicolumn{5}{|l|}{ Silver } \\
\hline \multicolumn{5}{|l|}{ Sodiun } \\
\hline \multicolumn{5}{|l|}{ Strontium } \\
\hline \multicolumn{5}{|l|}{ Thallium } \\
\hline \multicolumn{5}{|l|}{$\operatorname{Tin}$} \\
\hline \multicolumn{5}{|l|}{ Vanadium } \\
\hline Zinc & & & & \\
\hline
\end{tabular}


Suspended-sediment samples collected in water years 1994-95 were analyzed by the USGS for concentration. Some suspended-sediment samples also were analyzed for sand break (percent sand and percent silt/clay). Definitions of water-quality terms used in this report are provided in Appendix 2

("Glossary") at the back of this report.

\section{Sample Collection}

Some gaging stations wcre equipped with automatic samplers that collected composite water samples (that is, composite volumes of water pumped at discrete intervals) during a given period of flow. Following sample collection, USGS personncl retrieved the composite samples, reset the automatic sampler for subsequent sampling, and processed the chemical-quality and suspended-sediment samples. Chemical-quality samples were split from the composite samples. Suspended-sediment samples cither were split from the composite samples (identified as automatic) or were collected manually from flow in the channel (identified as manual). Additional information concerning sampling methods used by the USGS at the Site is provided by Stevens and others (1975), Guy and Norman (1982), Wershaw and others (1987), Britton and Greeson (1989), Fishman and Fricdman (1989), and EG\&G Rocky Flats, Incorporated (1992).

\section{Data Presentation}

Analytical results retrieved from the Sitc data base for chemical-quality samples collected by the USGS in water ycar 1994 are listed in table 41, and results for chemical-quality samples collected by the USGS in water year 1995 are listed in table 42 in the "Surface-Water Data" section. Analytical results for suspended-sediment samples collected by the USGS in water ycar 1994 are listed in table 43, and results for suspended-sediment samples collected by the USGS in water ycar 1995 are listed in table 44 in the "Surface-Water Data" section.

\section{REFERENCES CITED}

Benson, M.A., and Dalrymple, Tate, 1967, Gencral field and office procedures for indirect discharge measurements: U.S. Geological Survey Techniques of WaterResources Investigations, book 3, chap. A I, $30 \mathrm{p}$.
Bodhaine, G.L., 1968, Measurement of peak discharge at culverts by indirect methods: U.S. Geological Survey Techniques of Watcr-Resources Investigations, book 3, chap. A3, $60 \mathrm{p}$.

Britton, L.J., and Grecson, P.E., cds., 1989, Mcthods for collection and analysis of aquatic biological and microbiological samples: U.S. Geological Survey Techniques of Watcr-Resources Investigations, book 5 , chap. A4, 363 p. [Revised]

Carter, R.W., and Davidian, Jacob, 1968, General procedure fer gaging streams: U.S. Geological Survey Techniques of Watcr-Resources Investigations, book 3 , chap. A6, 13 p.

Dalrymple, Tate, and Benson, M.A., 1967, Measurement of peak discharge by the slope-area method: U.S. Geological Survey Techniques of Water-Resources Investigations, book 3, chap. A2, 12 p.

EG\&G Rocky Flats, Incorporated, 1992, Procedure FO.13 containerizing, preserving, handling, and shipping of soil and water samples: Golden, Colorado, Environmental Management Division Operating Procedures, v. 1 (Ficld Operations), p. 13-1-13-18.

EG\&G Rocky Flats, Incorporated, 1993a, Site environmental report for 1993: Golden, Colorado, RFP-ENV-93, $322 \mathrm{p}$.

EG\&G Rocky Flats, Incorporated, 1993b, Event-related surface-watcr monitoring report, Rocky Flats Plant, water years 1991 and 1992: Golden, Colorado, $146 \mathrm{p}$.

Fishman, M.J., and Friedman, L.C., eds., 1989, Methods for determination of inorganic substances in water and fluvial sediments ( $3 d$ ed.): U.S. Geological Survey Techniques of Water-Resources Investigations, book 5 , chap. A1, 545 p.

Guy, H.P., and Norman, V.W., 1982, Ficld methods for measurement of fluvial sediment: U.S. Geological Survey Techniques of Water-Resources Investigations, book 3 , chap. $\mathrm{C} 2,59 \mathrm{p}$. [4th printing].

Hem, J.D., 1985, Study and interpretation of the chemical characteristics of natural water ( $3 \mathrm{~d}$ ed.): U.S. Gcological Water-Supply Paper 2254, 263 p.

Rantz, S.E., and others, 1982, Measurement and computation of streamflow-v. 1, Measurement of stage and discharge; v. 2, Computation of discharge: U.S. Geological Survey Water-Supply Paper 2175, v. 1, p. 1-284; v. 2, p. 285-631.

Rocky Mountain Remediation Services, L.L.C., 1995, Event-rclated surface-water monitoring report, wateryear 1994: Golden, Colorado, $120 \mathrm{p}$. 
Stevens, H.H., Jr., Ficke, J.F., and Smoot, G.F., 1975, Water temperature - influential factors, field measurement, and data presentation: U.S. Geological Survey

Techniques of Water-Resources Investigations, book 1, chap. D1, $65 \mathrm{p}$.

Ugland, R.C., Maura, W.S., Steger, R.D., and O'Neill, G.B., 1995, Water resources data, Colorado, water year 1994 -v. 1, Missouri River basin, Arkansas River basin, and Rio Grande basin: U.S. Geological Survey Water-Data Report CO-94-1, 418 p.
U.S. Geological Survey, 1976, Hydrologic unit map, 1974, State of Colorado: U.S. Department of the Interior, Geological Survey, scale 1:500,000.

Wershaw, R.L., Fishman, M.J., Grabbe, R.R., and Lowe, L.E., eds., 1987, Methods for the determination of organic substances in water and fluvial sediments: U.S. Geological Survey Techniques of WaterResources Investigations, book 5, chap. A3, 80 p. 
8 


\section{Surface-Water Data}




\section{DAILY MEAN DISCHARGE DATA}

The following abbreviations are used in tables $4-40$. An explanation of the information and data presented in these tables is in Appendix 1 ("Discharge-Data Collection and Computation").

Lat is latitude;

long is longitude;

sec. is section;

$T$. is township;

$R$. is range;

$\mathrm{mi}^{2}$ is square miles;

$\mathrm{ft}$ is feet;

$\mathrm{ft}^{3} / \mathrm{s}$ is cubic feet per second;

MAX is the maximum daily mean discharge for a given month;

MIN is the minimum daily mean discharge for a given month;

AC-FT is acre-foot;

--- is a symbol used in place of daily mean discharge for periods of missing record or periods prior to gaging-station activation. 
Table 4.--Daily mean discharge, GS01 (Woman Creek at Indiana Street), water year 1994

WOMAN CREEK AT INDIANA ST.

SITE NUMBER. - - GS01

STATION IDENTIEICATION. - 395240105095500

LOCATION. - Lat $39^{\circ} 52^{\prime} 40^{\prime \prime}$, long $105^{\circ} 09^{\prime} 55^{\prime \prime}$, in $\mathrm{NE}^{1} / 4 \mathrm{NE}^{1} / 4 \mathrm{sec}$. 13, T. 2 S., R. 70 W., Jefferson County, Hydrologic Unit 10190003, Rocky Flats Environmental Technology Site, 100 feet upstream from Indiana street.

DRAINAGE AREA. - $2.16 \mathrm{mi}^{2}$.

PERIOD OF RECORD. - March 1994 to current year.

GAGE. - Water-stage recorder and Parshall flume. Elevation of gage is 5,622 ft above sea level.

REMARKS. - No estimated daily discharges. Records poor. Natural flow atfected by Mower Ditch diversion, approximately $1 / 4$ mile upstream.

DISCHARGE, CUBIC FEET PER SECOND, WATER YEAR 1994 ((X'T)'(JER 1993 TU SEP'JEMBER 1994)

\begin{tabular}{|c|c|c|c|c|c|c|c|c|c|c|c|c|}
\hline DAY & $\operatorname{coc}$ & NOV & Dec & JAN & PEB & MAL & APR & MAY & JUN & JUI, & AUG & SE.P \\
\hline 1 & $\cdots$ & $\cdots$ & $\cdots$ & $\cdots$ & $\ldots$ & $\cdots$ & .10 & .27 & .00 & .00 & .00 & .00 \\
\hline ? & $\cdots$ & $\ldots$ & $\ldots$ & $\ldots$ & $\ldots$ & $\ldots$ & .09 & .23 & .00 & .00 & .00 & .00 \\
\hline 3 & $\cdots$ & $\cdots$ & $\cdots$ & $\cdots$ & $\cdots$ & $\cdots$ & .08 & .21 & .00 & .00 & .00 & .00 \\
\hline 1 & $\cdots$ & $\ldots$ & $\cdots$ & $\ldots$ & $\ldots$ & $\cdots$ & .07 & .14 & .00 & .00 & .00 & .00 \\
\hline 5 & $\cdots$ & $\cdots$ & $\cdots$ & $\cdots$ & $\cdots$ & $\cdots$ & .11 & .10 & .00 & .00 & .00 & .00 \\
\hline 6 & $\cdots$ & $\cdots$ & $\cdots$ & $\cdots$ & $\cdots$ & $\cdots$ & .11 & .07 & .00 & .00 & .00 & .00 \\
\hline 7 & $\cdots$ & $\cdots$ & $\cdots$ & $\cdots$ & $\cdots$ & $\cdots$ & .08 & .06 & .00 & .00 & .00 & .00 \\
\hline 8 & $\cdots$ & $\cdots$ & $\cdots$ & $\cdots$ & $\ldots$ & $\cdots$ & .06 & .05 & .00 & .00 & .00 & .00 \\
\hline 9 & $\cdots$ & $\cdots$ & $\cdots$ & $\ldots$ & $\ldots$ & $\cdots$ & .10 & .05 & .00 & .00 & .00 & .00 \\
\hline 10 & $\cdots$ & $\cdots$ & $\cdots$ & $\cdots$ & $\cdots$ & $\cdots$ & .16 & .06 & .00 & .00 & .00 & .00 \\
\hline 11 & $\cdots$ & $\cdots$ & $\cdots$ & $\ldots$ & $\cdots$ & $\cdots$ & .17 & .03 & .00 & .00 & .00 & .00 \\
\hline 12 & $\cdots$ & $\cdots$ & $\cdots$ & $\cdots$ & $\ldots$ & $\cdots$ & .50 & .03 & .00 & .00 & .00 & .00 \\
\hline 13 & $\cdots$ & $\cdots$ & $\cdots$ & $\cdots$ & $\cdots$ & $\cdots$ & .73 & .06 & .00 & .00 & .00 & .00 \\
\hline 14 & $\cdots$ & $\ldots$ & $\cdots$ & $\ldots$ & $\ldots$ & $\ldots$ & .20 & .09 & .00 & .00 & .00 & .00 \\
\hline 15 & $\cdots$ & $\cdots$ & $\cdots$ & $\cdots$ & $\cdots$ & $\cdots$ & .13 & .03 & .00 & .00 & .00 & .00 \\
\hline 16 & $\cdots$ & $\cdots$ & $\ldots$ & $\ldots$ & $\cdots$ & $\ldots$ & .10 & .01 & .00 & .00 & .00 & .00 \\
\hline 17 & $\cdots$ & $\ldots$ & $\cdots$ & $\ldots$ & $\cdots$ & $\cdots$ & .09 & .01 & .00 & .00 & .00 & .00 \\
\hline 18 & $\cdots$ & $\cdots$ & $\cdots$ & $\cdots$ & $\cdots$ & $\cdots$ & .07 & .00 & .00 & .00 & .00 & .00 \\
\hline 19 & $\cdots$ & $\cdots$ & $\cdots$ & $\cdots$ & $\cdots$ & $\cdots$ & .06 & .00 & .00 & .00 & .00 & .00 \\
\hline 20 & $\cdots$ & $\cdots$ & $\cdots$ & $\cdots$ & $\cdots$ & $\cdots$ & .05 & .00 & .00 & .00 & .00 & .00 \\
\hline 21 & $\cdots$ & $\cdots$ & $\cdots$ & $\cdots$ & $\cdots$ & $\ldots$ & .04 & .00 & .00 & .00 & .00 & .00 \\
\hline 22 & $\cdots$ & $\cdots$ & $\cdots$ & $\cdots$ & $\cdots$ & .04 & .04 & .00 & .00 & .00 & .00 & .00 \\
\hline 23 & $\ldots$ & $\cdots$ & $\ldots$ & $\ldots$ & $\ldots$ & .03 & .03 & .00 & .00 & .00 & .00 & .00 \\
\hline 24 & $\cdots$ & $\cdots$ & $\cdots$ & $\ldots$ & $\cdots$ & .03 & .03 & .00 & .00 & .00 & .00 & .00 \\
\hline 25 & $\cdots$ & $\cdots$ & $\cdots$ & $\cdots$ & $\cdots$ & .04 & .22 & .00 & .00 & .00 & .00 & .00 \\
\hline 26 & $\cdots$ & $\cdots$ & $\cdots$ & $\cdots$ & $\cdots$ & .04 & .10 & .00 & .00 & .00 & .00 & .00 \\
\hline 27 & $\cdots$ & $\cdots$ & $\cdots$ & $\cdots$ & $\cdots$ & .05 & .09 & .00 & .00 & .00 & .00 & .00 \\
\hline 28 & $\cdots$ & $\cdots$ & $\cdots$ & $\cdots$ & $\cdots$ & .09 & .12 & .00 & .00 & .00 & .00 & .00 \\
\hline 29 & $\cdots$ & $\cdots$ & $\ldots$ & $\cdots$ & $\cdots$ & .11 & .27 & .00 & .00 & .00 & .00 & .00 \\
\hline 30 & $\cdots$ & $\cdots$ & $\cdots$ & $\cdots$ & $\cdots$ & .19 & .35 & .00 & .00 & .00 & .00 & .00 \\
\hline $3 i$ & $\cdots$ & $\cdots$ & $\cdots$ & $\cdots$ & $\cdots$ & .13 & $\cdots$ & .00 & $\cdots$ & .00 & .00 & $\cdots$ \\
\hline TOTAL & $\cdots$ & $\cdots$ & $\ldots$ & $\cdots$ & $\ldots$ & $\cdots$ & 4.35 & 1.50 & 0.00 & 0.00 & 0.00 & 0.00 \\
\hline MEAN & $\cdots$ & $\cdots$ & $\cdots$ & $\cdots$ & $\cdots$ & $\cdots$ & .14 & .048 & .000 & .000 & .000 & .000 \\
\hline $\operatorname{Max}$ & $\cdots$ & $\cdots$ & $\cdots$ & $\cdots$ & $\cdots$ & $\cdots$ & .73 & .27 & .00 & .00 & .00 & .00 \\
\hline MIN & $\cdots$ & $\cdots$ & $\cdots$ & $\cdots$ & $\cdots$ & $\cdots$ & .03 & .00 & .00 & .00 & .00 & .00 \\
\hline$A C-F T$ & $\cdots$ & $\cdots$ & $\cdots$ & $\cdots$ & $\cdots$ & $\cdots$ & 8.6 & 3.0 & .00 & .00 & .00 & .00 \\
\hline
\end{tabular}


SITE NUMBER. - - GS01

STATION IDENTIFICATION. - 395240105095500

LOCATION. - Lat $39^{\circ} 52^{\prime} 40^{\prime \prime}$, long $10 \mathrm{~b}^{\circ} 09^{\prime} 55^{\prime \prime}$, in $\mathrm{NE}^{\mathrm{i}} / 4 \mathrm{NE}^{1} / 4$ sec. $13, \mathrm{~T} .2 \mathrm{~S} ., \mathrm{R} .70 \mathrm{~W} ., \mathrm{Jefferson}$ Colinty, Hydrologic Unit 10190003, Rocky Flats Environmental Technology site, 100 feet upstream from Indiana street.

DRAINAGE AREA. - - $2.16 \mathrm{mi}^{2}$.

PERIOD JF RECORD. - March 1994 to current year.

GAGE. - Water-stage recorder and Parshall flume. Elevation of gage is $5,622 \mathrm{ft}$ above sea level.

REMARKS. - Records fair except for estimated daily discharges, which are poor. Natural flow affected by Mower Ditch diversion, approxinately $1 / 4 \mathrm{mile}$ upstream.

DISCHARGF, CUBIC FFET PER SECINIJ, WATER YFAR OCTOBER 1994 TOO SEPTEMBER 1995

\begin{tabular}{|c|c|c|c|}
\hline DAY & $\boldsymbol{O C T}$ & NOV & DEC \\
\hline 1 & .00 & .00 & .00 \\
\hline 2 & .00 & .00 & .00 \\
\hline 3 & .00 & .00 & .00 \\
\hline 4 & .00 & .00 & .00 \\
\hline 5 & .00 & .00 & .00 \\
\hline 6 & .00 & .00 & .00 \\
\hline 7 & .00 & .00 & .00 \\
\hline 8 & .00 & .00 & .00 \\
\hline 9 & .00 & .00 & .00 \\
\hline 10 & .00 & .00 & .00 \\
\hline 11 & .00 & .00 & .00 \\
\hline 12 & .00 & .00 & .00 \\
\hline 13 & .00 & .00 & .00 \\
\hline 14 & .00 & .00 & .00 \\
\hline 15 & .00 & .00 & .00 \\
\hline 16 & .00 & .00 & .00 \\
\hline 17 & .00 & .00 & .00 \\
\hline 18 & .00 & .00 & .00 \\
\hline 19 & .00 & .00 & .00 \\
\hline 20 & .00 & .00 & .00 \\
\hline 21 & .00 & .00 & .00 \\
\hline 22 & .00 & .00 & .00 \\
\hline 23 & .00 & .00 & .00 \\
\hline 24 & .00 & .00 & .00 \\
\hline 25 & .00 & .00 & .00 \\
\hline 26 & .00 & .00 & .00 \\
\hline 27 & .00 & .00 & .00 \\
\hline $2 \mathrm{~B}$ & .00 & .00 & .00 \\
\hline 29 & .00 & .00 & .00 \\
\hline 30 & .00 & .00 & .00 \\
\hline 31 & .00 & $\ldots$ & .00 \\
\hline TOTAL, & 0.00 & 0.00 & 0.00 \\
\hline MEAN & .000 & .000 & .000 \\
\hline MAX & .00 & .00 & .00 \\
\hline MIN & .00 & .00 & .00 \\
\hline$A C-F T$ & .00 & .00 & .00 \\
\hline
\end{tabular}
DAILY MEAAN VALUES

STATISTICS OF MONTHLY MEAN DATA FOR WATER YEARS 1994 - 1995, BY WATER YEAR (WY)

\begin{tabular}{|c|c|c|c|c|c|c|c|c|c|c|c|c|}
\hline MEAN & .000 & .000 & .000 & .000 & .010 & .027 & .40 & 2.26 & 1.19 & .002 & .004 & .000 \\
\hline $\operatorname{MAX}$ & .000 & .000 & .000 & .000 & .010 & .027 & .65 & 4.46 & 2.39 & .005 & .007 & .000 \\
\hline (WY) & 1995 & 1995 & 1995 & 1995 & 1995 & 1995 & 1995 & 1995 & 1995 & 1995 & 1995 & 1994 \\
\hline MIN & .000 & .000 & .000 & .000 & .010 & .027 & .14 & .048 & .000 & .000 & .000 & .000 \\
\hline (WY) & 1995 & 1995 & 1995 & 1995 & 1995 & 1995 & 1994 & 1994 & 1994 & 1994 & 1994 & 1994 \\
\hline
\end{tabular}

SUMMARY S'TATISTICS

ANNUAL TOTAL

ANNUAL MEAN

HIGHEST ANNUAL, MEAN

I,OWEST ANNUAL, MEAN

HIGHEST DAILY MEAN

LOWEST DAILY MEAN

ANNUAL SEVEN-DAY MINIMUM

ANNUAL RUNOFF (AC-FT)

10 PERCENT EXCEEDS

50 PERCFNT EXXEEDS

90 PERCENT EXCEEDS

$\begin{array}{ccc}\text { PEB } & \text { MAR } & \text { APR } \\ .00 & .01 & .0 \\ .00 & .02 & .04 \\ .00 & .02 & .04 \\ .00 & .02 & .04 \\ .00 & .02 & .04 \\ .00 & .03 & .03 \\ .00 & .03 & .03 \\ .00 & .07 & .03 \\ .00 & .06 & .03 \\ .00 & .04 & .05\end{array}$

.00
.00

.00

.03

.01
.04

.04

.02
.02

.02

.01

.01

.01

.01

.01

..

$\cdots$

0.27

.010
.04
.00

0.85
.027

.027

.07
.01

.01

.02

.03
.03

.05

.07

e1. 9

e 6.0
e1.

e3.8

19.6
.65
6.0
$\begin{array}{ll}A P R & M A Y \\ .05 & .69 \\ .04 & .4 \\ .04 & .4 \\ .04 & .4 \\ .04 & .32 \\ .03 & .2 \\ .03 & .2 \\ .03 & .2 \\ .03 & .1 \\ .05 & .12\end{array}$

MAY
.69
.47
.48
.41
.32
.26
.21
.21
.15
.12

JUN
.68
.53
5.6

JUL
.05
.01
.00
.00

$\begin{array}{ll}\text { AUG } & \text { SEP } \\ & \\ .20 & .00 \\ .01 & .00 \\ .01 & .00 \\ .00 & .00 \\ .00 & .00 \\ .00 & .00 \\ .00 & .00 \\ .00 & .00 \\ .00 & .00 \\ .00 & .00\end{array}$

37

.80
.30
3.9

3.7

2. 1

.69
.27

.27
.15

.15
.09

.05
.08

.11

.03

e2.

e1.0

e. 50

e. 30
e 1.1

e8.
2.0

.00

.00
.00

.00
.00
.00

e2. 5

1.1

e18

e4. 9

e9. 6

e3. 4

$\begin{array}{rrr}.63 & 138.36 & 71.56 \\ .65 & 4.46 & 2.39\end{array}$

6.0
.03

4.46
76
.06

.06

37
37
00

142

.00

.00
.04

.24

.09

PUR WATEF YEAR 1995

WATER YEARS 1994 - 1905
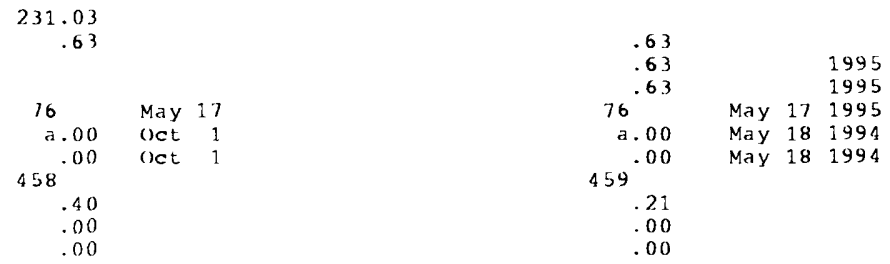

a No flow many days

e Estimated 
MOWER DITCH AT INDIANA ST.

SITE NUMBER. - - GS02

STATION IDENTIFICATION. - 395253105095500

LOCATION. - Lat $39^{\circ} 52^{\prime} 53^{\prime \prime}$, long $105^{\circ} 09^{\prime} 55^{\prime \prime}$, in $\mathrm{NE}^{\mathrm{i}} / 4 \mathrm{NE}^{1} / 4 \mathrm{sec}$. 13, T. 2 S., R. 70 w., Jefferson County, Hydrologic Unit 10190003, Rocky Flats Environmental Technology Site, 150 feet upstream from Indiana street.

DRAINAGE AREA. - - $1.66 \mathrm{mi}^{2}$.

PERIOD OF RECORD. - -March 1994 to current yoar.

GAGE. - Water-stage recorder and Parshall flume. Elevation of gage is 5,678 ft above sea level.

REMARKS. - Records fair. Flow in Mower Ditch is diverted from woman Creek, approximate 1 y $1 / 4 \mathrm{mile}$ upstream from station GS01 (395240105095500).

DISCHARGE, CUBIC FEET PER SECOND, WATER YEAR (KCTOBER 1993 TUO SEPTHMBER 7994

\begin{tabular}{|c|c|c|c|c|c|c|c|c|c|c|c|c|}
\hline DAY & $x+7$ & NOV & DEC & JAN & $F F B$ & MAR & $\mathrm{APR}$ & MAY & JUN & JUL, & AUG & SEP \\
\hline 1 & $\cdots$ & $\cdots$ & $\cdots$ & $\cdots$ & $\cdots$ & $\cdots$ & .41 & .89 & .00 & .00 & .00 & .00 \\
\hline 2 & $\cdots$ & $\cdots$ & $\cdots$ & $\cdots$ & $\cdots$ & $\cdots$ & .26 & .67 & .00 & .00 & .00 & .00 \\
\hline 3 & $\cdots$ & $\cdots$ & $\cdots$ & $\ldots$ & $\cdots$ & $\cdots$ & .24 & .70 & .00 & .00 & .00 & .00 \\
\hline 4 & $\ldots$ & $\ldots$ & $\cdots$ & $\ldots$ & $\cdots$ & $\cdots$ & .16 & .46 & .00 & .00 & .00 & .00 \\
\hline 5 & $\cdots$ & $\cdots$ & $\cdots$ & $\cdots$ & $\ldots$ & $\cdots$ & .18 & .43 & .00 & .00 & .00 & .00 \\
\hline 6 & $\ldots$ & $\ldots$ & $\ldots$ & $\ldots$ & $\ldots$ & $\cdots$ & .31 & .49 & .00 & .00 & .00 & .00 \\
\hline 7 & $\ldots$ & $\ldots$ & $\ldots$ & $\ldots$ & $\ldots$ & $\ldots$ & .42 & .40 & .00 & .00 & .00 & .00 \\
\hline 8 & $\ldots$ & $\ldots$ & $\ldots$ & $\cdots$ & $\ldots$ & $\cdots$ & .20 & .29 & .00 & .00 & .00 & .00 \\
\hline 9 & $\cdots$ & $\ldots$ & $\cdots$ & $\cdots$ & $\cdots$ & $\cdots$ & .34 & .28 & .00 & .00 & .00 & .00 \\
\hline 10 & $\cdots$ & $\cdots$ & $\cdots$ & $\ldots$ & $\cdots$ & $\cdots$ & 1.2 & .30 & .00 & .00 & .00 & .00 \\
\hline 11 & $\cdots$ & $\cdots$ & $\cdots$ & $\cdots$ & $\cdots$ & $\cdots$ & 1.1 & .17 & .00 & .00 & .00 & .00 \\
\hline 12 & $\cdots$ & $\cdots$ & $\cdots$ & $\cdots$ & $\cdots$ & $\cdots$ & 1.5 & .14 & .00 & .00 & .00 & .00 \\
\hline 13 & $\ldots$ & $\cdots$ & $\ldots$ & $\ldots$ & $\cdots$ & $\cdots$ & 1.2 & .15 & .00 & .00 & .00 & .00 \\
\hline 14 & $\ldots$ & $\ldots$ & $\ldots$ & $\ldots$ & $\ldots$ & $\ldots$ & .64 & .27 & .00 & .00 & .00 & .00 \\
\hline 15 & $\cdots$ & $\cdots$ & $\cdots$ & $\cdots$ & $\cdots$ & $\cdots$ & .47 & .12 & .00 & .00 & .00 & .00 \\
\hline 16 & $\ldots$ & $\ldots$ & $\cdots$ & $\cdots$ & $\ldots$ & $\cdots$ & .35 & .09 & .00 & .00 & .00 & .00 \\
\hline 17 & $\cdots$ & $\cdots$ & $\cdots$ & $\ldots$ & $\ldots$ & .10 & .27 & .04 & .00 & .00 & .00 & .00 \\
\hline 18 & $\ldots$ & $\cdots$ & $\ldots$ & $\ldots$ & $\cdots$ & .07 & .24 & .01 & .00 & .00 & .00 & .00 \\
\hline 19 & $\cdots$ & $\ldots$ & $\cdots$ & $\ldots$ & $\ldots$ & .05 & .19 & .00 & .00 & .00 & .00 & .00 \\
\hline 20 & $\cdots$ & $\ldots$ & $\ldots$ & $\ldots$ & $\ldots$ & .04 & .18 & .00 & .00 & .00 & .00 & .00 \\
\hline 21 & $\ldots$ & $\ldots$ & $\ldots$ & $\ldots$ & $\ldots$ & .05 & .17 & .00 & .00 & .00 & .00 & .00 \\
\hline 22 & $\ldots$ & $\ldots$ & $\cdots$ & $\ldots$ & $\cdots$ & .04 & .14 & .00 & .00 & .00 & .00 & .00 \\
\hline 23 & $\ldots$ & $\cdots$ & $\cdots$ & $\ldots$ & $\cdots$ & .02 & .14 & .00 & .00 & .00 & .00 & .00 \\
\hline 24 & $\ldots$ & $\ldots$ & $\ldots$ & $\ldots$ & $\ldots$ & .00 & .11 & .00 & .00 & .00 & .00 & .00 \\
\hline 25 & $\cdots$ & $\cdots$ & ... & $\cdots$ & $\cdots$ & .00 & .37 & .00 & .00 & .00 & .00 & .00 \\
\hline 26 & $\cdots$ & $\cdots$ & $\cdots$ & $\ldots$ & $\ldots$ & .01 & .52 & .01 & .00 & .00 & .00 & .00 \\
\hline 27 & $\cdots$ & $\ldots$ & $\ldots$ & $\ldots$ & $\ldots$ & .06 & .47 & .01 & .00 & .00 & .00 & .00 \\
\hline 28 & $\cdots$ & $\ldots$ & $\ldots$ & $\cdots$ & $\ldots$ & .09 & .55 & .00 & .00 & .00 & .00 & .00 \\
\hline 29 & $\cdots$ & $\cdots$ & $\cdots$ & $\cdots$ & $\ldots$ & .42 & .82 & .01 & .00 & .00 & .00 & .00 \\
\hline 30 & $\cdots$ & $\ldots$ & $\ldots$ & $\ldots$ & $\ldots$ & .42 & 1.4 & .00 & .00 & .00 & .00 & .00 \\
\hline 31 & $\cdots$ & $\cdots$ & $\cdots$ & $\ldots$ & $\cdots$ & .64 & $\cdots$ & .00 & $\cdots$ & .00 & .00 & $\therefore$ \\
\hline TOTAL & $\cdots$ & $\ldots$ & $\cdots$ & $\ldots$ & $\cdots$ & $\ldots$ & 14.55 & 5.93 & 0.00 & 0.00 & 0.00 & 0.00 \\
\hline MEAN & $\ldots$ & $\ldots$ & $\cdots$ & $\ldots$ & $\cdots$ & $\ldots$ & .48 & .19 & .000 & .000 & .000 & .000 \\
\hline $\operatorname{MAX}$ & $\ldots$ & $\ldots$ & $\cdots$ & $\cdots$ & $\ldots$ & $\cdots$ & 1.5 & .89 & .00 & .00 & .00 & .00 \\
\hline MIN & $\cdots$ & $\ldots$ & $\cdots$ & $\ldots$ & $\ldots$ & $\ldots$ & .11 & .00 & .00 & .00 & .00 & .00 \\
\hline$A C \cdot F T$ & $\cdots$ & $\cdots$ & $\cdots$ & $\ldots$ & $\ldots$ & $\cdots$ & 29 & 12 & .00 & .00 & .00 & .00 \\
\hline
\end{tabular}


MOWER DITCH AT INDIANA ST.

SITE NUMBER. - GSO2

STATION IDENTIFICATION. - 395253105095500

LOCATION. - Lat $39^{\circ} 52^{\prime} 53^{\prime \prime}$, long $105^{\circ} 09^{\prime} 55^{\prime \prime}$, in $\mathrm{NE}^{1} / 4 \mathrm{NE}^{1} / 4 \mathrm{sec}$. $13, \mathrm{~T}$. 2 S. R. 70 W. Jefferson County, Hydrologic Unit 10190003, Rocky Flats Environmental Technology site, 150 feet upstream from Indiana st.

DRAINAGE AREA. $-1.66 \mathrm{mi}^{2}$.

PERTOD OF RECORD. - March 1994 to current year

GAGE. - Water-stage recorder and Parshall flume. Elevation of gage is $5,678 \mathrm{ft}$ above sea level.

REMARKS. - Records fair except for estimated daily discharges, which are poor. Flow in Mower Ditch diverted from woman creek, approximately $1 / 4$ mile upstream from station Gs01 (395240105095500). DISCHARGE, CUBIC FEET PER SECOND, WATER YEAR OCTOBER :994 TO SEPTEMBER 1995

\begin{tabular}{|c|c|c|c|c|c|c|c|c|c|c|c|c|}
\hline DAY & OCT & NOV & DEC & JAN & FEB & MAR & A PR & MAY & JUN & JUL, & AUG & SEP \\
\hline 1 & .00 & .00 & .00 & .00 & .22 & .00 & .16 & 2.6 & 3.3 & .19 & e4. 5 & .00 \\
\hline 2 & .00 & .00 & .03 & .00 & .12 & .01 & .07 & 1.6 & 2.6 & .09 & e 1.0 & .00 \\
\hline 3 & .00 & .00 & .02 & .00 & .04 & .03 & .04 & 2.9 & 5.4 & .04 & e. 50 & .00 \\
\hline 4 & .00 & .00 & .01 & .00 & .03 & .07 & .02 & 3.4 & 3.6 & .02 & .00 & .00 \\
\hline 5 & .00 & .00 & .00 & .00 & .02 & .03 & .01 & 1.3 & 3.4 & .01 & .00 & .00 \\
\hline 6 & .00 & .00 & .00 & .00 & .01 & .05 & .00 & .60 & 1.2 & .00 & .00 & .00 \\
\hline 7 & .00 & .00 & .00 & .00 & .01 & .05 & .00 & .30 & .46 & .00 & .00 & .00 \\
\hline 8 & .00 & .00 & .00 & .00 & .01 & .06 & .00 & .32 & 3.3 & .00 & .00 & .00 \\
\hline 9 & .00 & .00 & .00 & .02 & .01 & .20 & .00 & .30 & 8.2 & .00 & .00 & .00 \\
\hline 10 & .00 & .00 & .00 & .08 & .00 & .22 & .03 & .17 & 3.4 & .00 & .00 & .00 \\
\hline 11 & .00 & .00 & .00 & .07 & .00 & .07 & .10 & .12 & 2.5 & .00 & .00 & .00 \\
\hline 12 & .00 & .00 & .00 & .02 & .00 & .03 & .58 & .13 & .98 & .00 & .00 & .00 \\
\hline 13 & .00 & .00 & .00 & .01 & .00 & .00 & .70 & .06 & .39 & .00 & .00 & .00 \\
\hline 14 & .00 & .00 & .00 & .01 & .07 & .00 & .21 & .05 & .29 & .00 & .00 & .00 \\
\hline 15 & .00 & .01 & .00 & .01 & .19 & .00 & .09 & .05 & .18 & .00 & .00 & .00 \\
\hline 16 & .00 & .02 & .00 & .01 & .15 & .00 & .06 & .04 & .10 & .00 & .00 & .00 \\
\hline 17 & .00 & .03 & .00 & .00 & .13 & .00 & .53 & 8.8 & .12 & .00 & .00 & .00 \\
\hline 18 & .00 & .00 & .00 & .00 & .32 & .02 & .78 & 6.8 & .26 & .00 & .00 & .00 \\
\hline 19 & .00 & .00 & .00 & .00 & .29 & .00 & 2.6 & 4.7 & .09 & .00 & .00 & .00 \\
\hline 20 & .00 & .02 & .00 & .00 & .16 & .00 & 3.1 & 3.1 & .05 & .00 & .00 & .00 \\
\hline 21 & .00 & .02 & .00 & .00 & .09 & .00 & 2.9 & 2.0 & .02 & .00 & .00 & .00 \\
\hline 22 & .00 & .01 & .00 & .00 & .08 & .00 & 4.6 & .87 & .02 & .00 & .00 & .00 \\
\hline 23 & .00 & .02 & .00 & .00 & .07 & .00 & 4.3 & 3.9 & .01 & .00 & .00 & .00 \\
\hline 24 & .00 & .03 & .01 & .00 & .03 & .00 & 5.4 & 7.8 & .03 & .00 & .00 & .00 \\
\hline 25 & .00 & .04 & .02 & .00 & .02 & .00 & 3.8 & 5.1 & .02 & .00 & .00 & .00 \\
\hline 26 & .00 & .04 & .02 & .00 & .01 & .00 & 6.5 & 4.3 & .00 & .00 & .00 & .00 \\
\hline 27 & .00 & .01 & .02 & .01 & .00 & .01 & e6.7 & 8.7 & .00 & .00 & .00 & .00 \\
\hline 28 & .00 & .00 & .01 & .01 & .01 & .05 & 5.1 & 6.2 & .03 & .00 & .00 & .00 \\
\hline 29 & .00 & .00 & .01 & .02 & $\cdots$ & .09 & $e 6.9$ & 8.0 & 1.3 & 1.5 & .00 & .00 \\
\hline 30 & .00 & .00 & .01 & .03 & $\cdots$ & .09 & 6.5 & 6.3 & .37 & 8.1 & .00 & .00 \\
\hline 31 & .00 & $\cdots$ & .00 & .06 & $\cdots$ & .19 & $\ldots$ & 4.1 & $\cdots$ & 6.5 & .00 & $\cdots$ \\
\hline TOTAL & 0.00 & 0.25 & 0.16 & 0.36 & 2.09 & 1.27 & 61.78 & 94.61 & 41.62 & 26.45 & 6.00 & 0.00 \\
\hline MEAN & .000 & .008 & .005 & .012 & .075 & .041 & 2.06 & 3.05 & 1.39 & .53 & .19 & .000 \\
\hline $\operatorname{MAX}$ & .00 & .04 & .03 & .08 & .32 & .22 & 6.9 & 8.8 & 8. 2 & $8 . i$ & 4.5 & .00 \\
\hline MIN & .00 & .00 & .00 & .00 & .00 & .00 & .00 & .04 & .00 & .00 & .00 & .00 \\
\hline$A C-F_{T}^{T}$ & .00 & .5 & .3 & .7 & 4.1 & 2.5 & 123 & 188 & 83 & 33 & 12 & .00 \\
\hline
\end{tabular}

STATISTICS OF MONTHLY MEAN DATA FOR WATER YEARS 1994 - 1995, BY WATER YEAR (WY)

$\begin{array}{llllllllllrrr}\text { MEAN } & .000 & .008 & .005 & .012 & .075 & .041 & 1.27 & 1.62 & .69 & .27 & .097 & .000 \\ \text { MAX } & .000 & .008 & .005 & .012 & .075 & .041 & 2.06 & 3.05 & 1.39 & .53 & .19 & .000 \\ \text { (WY) } & 1995 & 1995 & 1995 & 1995 & 1995 & 1995 & 1995 & 1995 & 1995 & 1995 & 1995 & 1994 \\ \text { MIN } & .000 & .008 & .005 & .012 & .075 & .041 & .48 & .19 & .000 & .000 & .000 & .000 \\ \text { (WY) } & 1995 & 1995 & 1995 & 1995 & 1995 & 1995 & 1994 & 1994 & 1994 & 1994 & 1994 & 1994\end{array}$

SUMMARY STATISTICS

FOR 1995 WATER YEAR

WATER YEARS 1994 - 1995

ANNUAL TOTAL

ANNUAL MEAN

HIGHEST ANNUAL, MEAN

LOWEST ANNUAL MEAN

HIGHEST DAILY MEAN

I,OWEST DAILY MEAN

ANNUAL SEVEN-DAY MINIMUM

ANNUAL RUNOFF (AC-FT)

10 PERCENT EXCEEDS

50 PERCENT EXCEEDS

90 PERCENT EXCEEDS

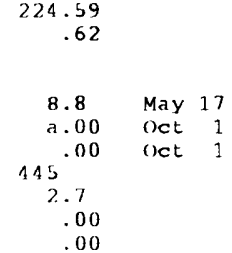

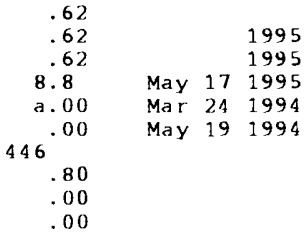

a No flow many days

e Estimated 
Table 8.--Daily mean discharge, GSO3 (Walnut Creek at Indiana Street), water year 1994

WALNUT CREEK AT INDIANA ST.

SITE NUMBER. - - GS03

STATION IDENTIFICATION. - 395407105095900

LOCATION. - Lat $39^{\circ} 54^{\prime} 07^{\prime \prime}$, long $105^{\circ} 09^{\prime} 59^{\prime \prime}$, in SE $\mathrm{SE}^{1} / 4 \mathrm{SE}^{1} / 4$ sec.1, T.2 S., R. 70 W., Jefferson County, Hydrologic Unit 10190003 , Rocky Flats Environmental Technology site, 300 feet upstream from Indiana street.

DRAINAGE AREA. - $2.70 \mathrm{mi}^{2}$, of which $0.91 \mathrm{mi}^{2}$ is noncontributing.

PERIOD OF RECORD. - March 1994 to current year.

GAGE. - Water-stage recorder and parallel Parshall flumes. Elevation of gage is $5,635 \mathrm{ft}$ above sea level.

REMARKS. - Records poor. No flow data collected by USGS April 19 to May 13 ; records for this period published by Rocky Mountain Remediation services, L.L.C. (1995). Most flow in lower Walnut Creek drainage regulated by the A-series ponds.

DISCHARGE, CUBIC FEET PER SECUND, WATER YEAR OCTUBER 1993 T'O SEPTEMBER 1994

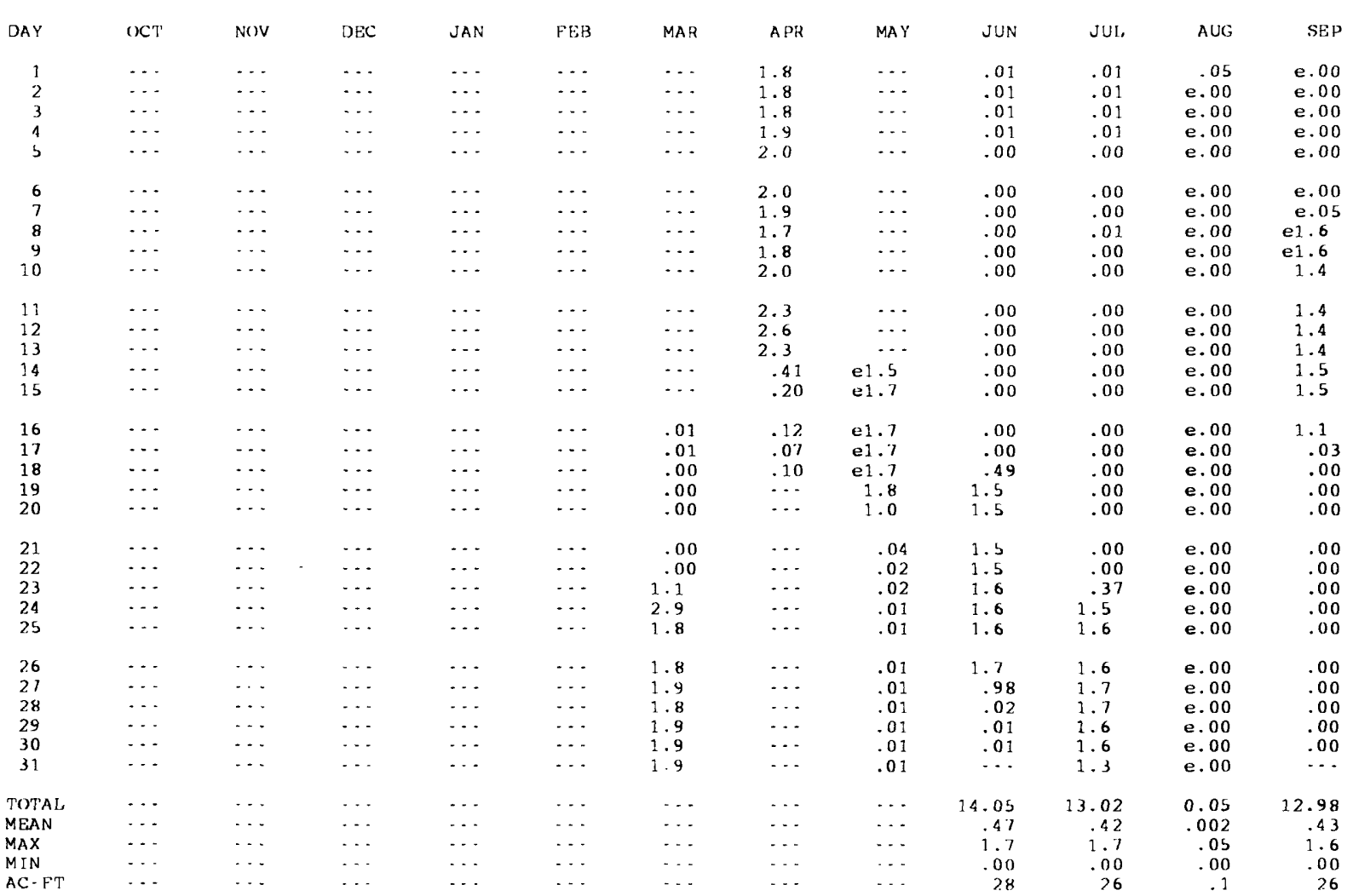

e Estimated 
WALNUT CREEK AT INDIANA ST.

SITE NUMBER. - - GS03

STATION IDENTIFICATION. - 395407105095900

LOCATION. - Lat $39^{\circ} 54^{\prime} 07^{\prime \prime}$, long $105^{\circ} 09^{\prime} 59^{\prime \prime}$, in SE $/ 4 \mathrm{SE}^{1} / 4$ sec.1, T.2 S., R. 70 W., Jefferson County, Hydrologic Unit 10190003, Rocky Flats Environmental Technology site, 300 feet upstream from Indiana street.

DRAINAGE AREA. - - 2.70 $\mathrm{mi}^{2}$, of which $0.91 \mathrm{mi}^{2}$ is noncontributing.

PERIOD OF RECOPD. - March 1994 to current year.

GAGE. - Water-stage recorder and parallel Parshall flumes. Elevation of gage is 5,635 ft above sea level.

REMARKS. - Records poor. Most flow in lower walnut Creek drainage regulated by the A-Series ponds. DISCHARGE, CUBIC FEET PER SECUND, WATER YFAR (KTOBER 2994 TU SEPTEMBER 1995

\begin{tabular}{|c|c|c|c|c|c|c|c|c|c|c|c|c|}
\hline DAY & $n C^{T}$ & $\mathrm{NOV}$ & DEC & JAN & FEB & MAR & A PR & MAY & JUN & JUL & AUG & SEP \\
\hline 1 & .00 & .07 & .00 & .55 & e. 00 & .01 & .01 & 9.6 & 9.8 & .02 & .00 & .00 \\
\hline 2 & .00 & .05 & .00 & .02 & .00 & .01 & .01 & 14 & 8.7 & .02 & 2.0 & .00 \\
\hline 3 & .00 & .06 & .00 & .11 & .00 & .02 & .01 & 18 & 7.7 & .02 & 2.5 & .00 \\
\hline 4 & .00 & .05 & .00 & .02 & .00 & .01 & .01 & i 8 & 6.7 & .02 & 2.5 & .00 \\
\hline 5 & .00 & .05 & .00 & e. 01 & .00 & .01 & .01 & 16 & 6.1 & .02 & 2.1 & .00 \\
\hline 6 & .02 & .04 & .00 & e.01 & .00 & .01 & .01 & $: 6$ & 6.2 & .02 & 2.2 & .00 \\
\hline 7 & .00 & .03 & .00 & e.01 & .00 & .01 & .01 & 16 & 6.2 & .02 & 2.1 & .00 \\
\hline 8 & .00 & .00 & .00 & e. 01 & .83 & .01 & .01 & 16 & 6.2 & .02 & 1.7 & .00 \\
\hline 9 & .00 & .00 & e. 00 & e. 01 & 2.0 & .00 & .01 & 17 & 6.2 & .02 & 1.7 & .00 \\
\hline 10 & .00 & .00 & e. 00 & e.01 & 2.0 & .77 & .01 & 17 & 6.2 & .02 & 1.6 & .00 \\
\hline 11 & e. 00 & .00 & e. เo & e.01 & 1.9 & 1.6 & .01 & 17 & 6.2 & .01 & 1.4 & .00 \\
\hline 12 & e. 00 & .00 & $\mathrm{e} 2.1$ & e. 01 & 1.7 & 1.4 & .01 & 17 & es. 0 & .00 & 1.1 & .00 \\
\hline 13 & e. 00 & .01 & e2.1 & e.01 & 1.5 & 1.3 & .01 & 17 & e 4.0 & .00 & .76 & .00 \\
\hline 14 & e. 00 & .04 & e2.0 & e. 01 & .08 & 1.3 & .82 & 17 & e4.0 & .01 & .02 & .00 \\
\hline 15 & e. 00 & .04 & e1.9 & e.01 & .04 & 1.1 & 1.8 & 17 & e4. 0 & .00 & .01 & 1.8 \\
\hline 16 & e. 00 & .04 & 2.1 & e. 01 & .03 & .98 & 1.7 & 19 & e2.0 & .00 & .00 & 3.0 \\
\hline 17 & e. 00 & .02 & 2.2 & e.01 & .03 & .66 & 1.5 & e 60 & .05 & .00 & .00 & 2.7 \\
\hline 18 & e. 00 & .00 & 2.2 & e.01 & .03 & .03 & 1.3 & 12 & .05 & .00 & .00 & 2.5 \\
\hline 19 & e. 00 & .00 & 1.6 & e. 01 & .03 & .01 & 2.9 & 12 & .05 & .00 & .01 & 2.4 \\
\hline 20 & e. 00 & .01 & 1.6 & e. 01 & .02 & .01 & 2.9 & 12 & .05 & .01 & .01 & 2.2 \\
\hline 21 & e. 00 & .00 & 1.5 & e. 01 & .02 & .01 & 2.3 & 12 & .04 & .00 & .01 & 2.3 \\
\hline 22 & .31 & .00 & .84 & e.01 & .02 & .01 & 3.0 & 12 & .04 & .00 & .01 & 2.0 \\
\hline 23 & 1.5 & .00 & .06 & e.01 & .02 & .01 & 2.3 & 12 & .03 & .00 & .01 & 1.6 \\
\hline 24 & 1.7 & .00 & .04 & e. 01 & .02 & .01 & 3.1 & 12 & .03 & .00 & .01 & 1.6 \\
\hline 25 & 2.0 & .00 & .03 & e. 00 & .02 & .01 & 1.4 & 12 & .02 & .00 & .01 & 1.3 \\
\hline 2.6 & 2.4 & .00 & .03 & e.00 & .02 & .01 & 7.2 & 12 & .02 & .00 & .01 & 1.2 \\
\hline 27 & 2.3 & .04 & .03 & e.00 & .01 & .01 & 11 & 12 & .02 & .00 & .00 & .77 \\
\hline 28 & 2.1 & .00 & .03 & e. 00 & .01 & .01 & 11 & 12 & .02 & .00 & .00 & .01 \\
\hline 29 & 2.1 & .00 & .03 & e. 00 & $\cdots$ & .01 & 17 & 12 & .02 & .00 & .00 & .00 \\
\hline 30 & 2.1 & .00 & .03 & e. 00 & $\cdots$ & .01 & 14 & 12 & .02 & .00 & .00 & .00 \\
\hline 31 & 2.4 & $\cdots$ & .62 & e. 00 & $\cdots$ & .01 & $\cdots$ & 11 & $\cdots$ & .00 & .00 & $\cdots$ \\
\hline TOTAI, & 17.93 & 0.55 & 21.54 & 0.90 & 10.33 & 9.36 & 85.35 & 488.6 & 95.66 & 0.23 & 21.77 & 25.38 \\
\hline MEAN & .58 & .018 & .69 & .029 & .37 & .30 & 2.84 & 15.8 & 3.19 & .007 & .70 & .85 \\
\hline $\operatorname{MAX}$ & 2.4 & .07 & 2.2 & .55 & 2.0 & 1.6 & 17 & 60 & 9.8 & .02 & 2.5 & 3.0 \\
\hline MIN & .00 & .00 & .00 & .00 & .00 & .00 & .01 & 9.6 & .02 & .00 & .00 & .00 \\
\hline$A C \cdot F T$ & 36 & 1.1 & 43 & 1.8 & 20 & 19 & 169 & 969 & 190 & .5 & 43 & 50 \\
\hline
\end{tabular}

STATISTICS OF MONTHLY MEAN DATA FOR WATER yEARS 1994 - 1995, BY WATER YEAR (WY)

$\begin{array}{lrrrr}\text { MEAN } & .58 & .018 & .69 & .029 \\ \text { MAX } & .58 & .018 & .69 & .029 \\ \text { (WY) } & 1995 & 1995 & 1995 & 1995 \\ \text { MIN } & .58 & .018 & .69 & .029 \\ \text { (WY) } & 1995 & 1995 & 1995 & 1995\end{array}$

$\begin{array}{rrrr}.37 & .30 & 1.87 & 8.06 \\ .31 & .30 & 2.84 & 15.8 \\ 1995 & 1995 & 1995 & 1995 \\ .31 & .30 & .89 & .36 \\ 1995 & 1995 & 1994 & 1994\end{array}$

1.83
3.19
1995
.47
1994
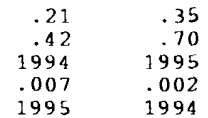

.64
.85
1995
.43
1994

SUMMARY STATISTICS

FOR 1995 WATER YEAR

WATER YEARS 1994 - 1995

ANNUAL, TUTAL,

$$
777.60
$$

HIGHEST ANNUAL MEAN

LOWEST ANNUAL MEAN

HIGHEST DA]LY MEAN

LOWEST DAILY MEAN

ANNUAL SEVEN-DAY MINIMUM

ANNUAL RUNOFF (AC-FT)

10 PERCENT EXCEEDS

50 PERCENT EXCEEDS

90 PERCENT EXCEFDS
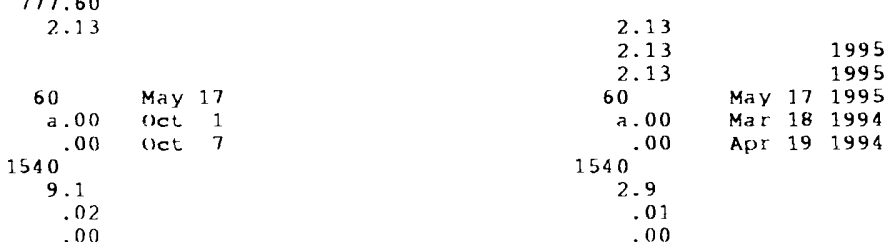

a No flow many days

e Estimater 
Table 10.--Daily mean discharge, GS05 (North Worman Creek at West Buffer Zone Fence Line), water year 1994

NORTH WOMAN CREEK AT WEST BUFFER ZONE FENCE LINE

SITE NUMBER. - GSO5

STATION IDENTIFICATION. - 395306105131700

LOCATION. - Lat $39^{\circ} 53^{\prime} 06^{\prime \prime}$, long $105^{\circ} 13^{\prime} 17^{\prime \prime}$, in $\mathrm{NW}^{1} / 4 \mathrm{NW}^{1} / 4$ sec. $15, \mathrm{~T} .2 \mathrm{~S} ., \mathrm{R} .70$ w., Jefferson County, Hydrologic Unit 10190003, Rocky Flats Environmental Technology site, about 200 feet downstream from west site fence line.

DRAINAGE AREA. - $0.20 \mathrm{mi}^{2}$.

PE'.IOD UF RECORD. - March 1994 to current year.

GAGE. - Water-stage recorder and Parshall flume. Elevation of gage is $6,039 \mathrm{ft}$ above sea level.

REMARKS. - - Records poor. DISCHARGE, CUBIC PEET PER SECOND, WATER YEAR OCTOBER 1993 TO SEPTEMBER 1994
DAILY MEAN VALUES

\begin{tabular}{|c|c|c|c|c|c|c|c|c|c|c|c|c|}
\hline DAY & (xT & $\mathrm{N}(\mathrm{IV}$ & DEC & JAN & $\mathrm{FEB}$ & MAR & APR & MAY & JUN & JUL & AUG & SEP \\
\hline 1 & $\cdots$ & $\cdots$ & $\cdots$ & $\cdots$ & $\cdots$ & $\cdots$ & .12 & .54 & .07 & .00 & .05 & .00 \\
\hline 2 & $\cdots$ & $\cdots$ & $\cdots$ & $\cdots$ & $\cdots$ & $\cdots$ & .10 & .73 & .07 & .00 & .00 & .00 \\
\hline 3 & $\ldots$ & $\ldots$ & $\ldots$ & $\ldots$ & $\cdots$ & $\ldots$ & .07 & .52 & .10 & .00 & .00 & .00 \\
\hline 4 & $\cdots$ & $\cdots$ & $\cdots$ & $\cdots$ & $\cdots$ & $\cdots$ & .06 & .46 & .08 & .00 & .01 & .00 \\
\hline 5 & $\cdots$ & $\cdots$ & $\cdots$ & $\cdots$ & $\cdots$ & $\cdots$ & .11 & .37 & .06 & .00 & .01 & .00 \\
\hline 6 & $\ldots$ & $\cdots$ & $\cdots$ & $\cdots$ & $\cdots$ & $\cdots$ & .17 & .31 & .07 & .00 & .01 & .00 \\
\hline 7 & $\cdots$ & $\cdots$ & $\cdots$ & $\cdots$ & $\cdots$ & $\cdots$ & .10 & .26 & .08 & .00 & .00 & .00 \\
\hline 8 & $\cdots$ & $\cdots$ & $\cdots$ & $\cdots$ & $\cdots$ & $\cdots$ & .05 & .22 & .10 & .00 & .01 & .00 \\
\hline 9 & $\cdots$ & $\cdots$ & $\cdots$ & $\cdots$ & $\cdots$ & $\cdots$ & .09 & .22 & .19 & .00 & .01 & .00 \\
\hline 10 & $\cdots$ & $\cdots$ & $\cdots$ & $\cdots$ & $\cdots$ & $\cdots$ & .27 & .23 & .26 & .00 & .03 & .00 \\
\hline 11 & $\cdots$ & $\cdots$ & $\ldots$ & $\cdots$ & $\cdots$ & $\ldots$ & .31 & .18 & .29 & .00 & .03 & .00 \\
\hline 12 & $\cdots$ & $\cdots$ & $\ldots$ & $\cdots$ & $\cdots$ & $\cdots$ & 1.3 & .15 & .24 & .00 & .02 & .00 \\
\hline 13 & $\cdots$ & $\cdots$ & $\cdots$ & $\cdots$ & $\cdots$ & $\cdots$ & .57 & .17 & .22 & .00 & .02 & .00 \\
\hline 14 & $\cdots$ & $\cdots$ & $\cdots$ & $\cdots$ & $\cdots$ & $\cdots$ & .34 & .14 & .21 & .00 & .02 & .00 \\
\hline 15 & $\cdots$ & $\cdots$ & $\cdots$ & $\cdots$ & $\cdots$ & $\cdots$ & .26 & .07 & .22 & .00 & .00 & .00 \\
\hline 16 & $\cdots$ & $\cdots$ & $\cdots$ & $\cdots$ & $\cdots$ & $\cdots$ & .20 & .05 & .26 & .00 & .00 & .00 \\
\hline 11 & $\cdots$ & $\cdots$ & $\cdots$ & $\cdots$ & $\cdots$ & $\ldots$ & .19 & .03 & .28 & .00 & .00 & .00 \\
\hline 18 & $\cdots$ & $\cdots$ & $\cdots$ & $\cdots$ & $\cdots$ & $\ldots$ & .18 & .03 & .27 & .00 & .00 & .00 \\
\hline 19 & $\cdots$ & $\cdots$ & $\cdots$ & $\cdots$ & $\cdots$ & $\cdots$ & .19 & .03 & .15 & .00 & .00 & .00 \\
\hline 20 & $\cdots$ & $\cdots$ & $\cdots$ & $\cdots$ & $\cdots$ & $\cdots$ & .17 & .04 & .07 & .00 & .00 & .00 \\
\hline 21 & $\cdots$ & $\cdots$ & $\cdots$ & $\ldots$ & $\cdots$ & $\ldots$ & .14 & .13 & .01 & .00 & .00 & .00 \\
\hline 22 & $\cdots$ & $\cdots$ & $\cdots$ & $\cdots$ & $\cdots$ & .29 & .14 & .13 & .00 & .00 & .00 & .00 \\
\hline 23 & $\cdots$ & $\cdots$ & $\cdots$ & - . & $\cdots$ & .22 & .13 & .11 & .00 & .00 & .00 & .00 \\
\hline 24 & $\cdots$ & $\cdots$ & $\cdots$ & $\cdots$ & $\cdots$ & .21 & .09 & .14 & .01 & .00 & .00 & .00 \\
\hline 25 & $\cdots$ & $\cdots$ & $\cdots$ & $\cdots$ & $\cdots$ & .18 & .32 & .14 & .00 & .02 & .00 & .00 \\
\hline 26 & $\cdots$ & $\cdots$ & $\ldots$ & $\cdots$ & $\cdots$ & .16 & .22 & .10 & .00 & .04 & .00 & .00 \\
\hline 27 & $\cdots$ & $\cdots$ & $\cdots$ & $\cdots$ & $\cdots$ & .16 & .31 & .09 & .00 & .06 & .00 & .00 \\
\hline 28 & $\cdots$ & $\cdots$ & $\cdots$ & $\cdots$ & $\cdots$ & .22 & .32 & .10 & .00 & .06 & .00 & .00 \\
\hline 29 & $\cdots$ & $\cdots$ & $\cdots$ & $\cdots$ & $\cdots$ & .25 & .69 & .03 & .00 & .07 & .00 & .00 \\
\hline 30 & $\cdots$ & $\cdots$ & $\cdots$ & $\cdots$ & $\cdots$ & .33 & 1.4 & .03 & .00 & .09 & .00 & .00 \\
\hline 31 & $\cdots$ & $\cdots$ & $\cdots$ & $\cdots$ & $\cdots$ & .21 & $\cdots$ & .06 & $\cdots$ & .08 & .00 & $\cdots$ \\
\hline TOTAL, & $\cdots$ & $\cdots$ & $\cdots$ & $\cdots$ & $\cdots$ & $\cdots$ & 8.61 & 5.81 & 3.31 & 0.42 & 0.22 & 0.00 \\
\hline MEAN & $\cdots$ & $\cdots$ & $\cdots$ & $\cdots$ & $\cdots$ & $\cdots$ & .29 & .19 & .11 & .014 & .007 & .000 \\
\hline $\operatorname{MAX}$ & $\cdots$ & $\cdots$ & $\cdots$ & $\cdots$ & $\cdots$ & $\cdots$ & 1.4 & .73 & .29 & .09 & .05 & .00 \\
\hline MIN & $\cdots$ & $\cdots$ & $\cdots$ & $\cdots$ & $\cdots$ & $\cdots$ & .05 & .03 & .00 & .00 & .00 & .00 \\
\hline $\mathrm{AC} \cdot \mathrm{FT}$ & $\cdots$ & $\cdots$ & $\cdots$ & $\cdots$ & $\cdots$ & $\cdots$ & 17 & 12 & 6.6 & .8 & .4 & .00 \\
\hline
\end{tabular}


NORTH WOMAN CREEK AT WEST BUFFER ZONE FENCE LINE

SITE NUMBER. - - GS05

STATION IDENTIFICATION. - 395306105131700

LOCATION. - Lat $39^{\circ} 53^{\prime} 06^{\prime \prime}$, long $105^{\circ} 13^{\prime} 17^{\prime \prime}$, in $\mathrm{NW}^{1} / 4 \mathrm{NW}^{1} / 4$ sec. 15 , T. 2 S., R. 70 W., Jefferson County, Hydrologic Unit 10190003, Rocky Flats Environmental Technology Site, about 200 feet downstream from west site fence line.

DRAINAGE AREA. - $0.20 \mathrm{mi}^{2}$.

PERIOD OF RECORD. - Marc 1994 to current year.

GAGE. - Water-stage recorder and Parshall flume. Elevation of gage is $6,039 \mathrm{ft}$ above sea level.

REMARKS.--Records fair except for Apr 27, 29 and estimated daily discharges, which are poor. DisCHARGE, CUBIC FEET PER SECOND, WATER YEAR OCTOBER 1994 TO SEPTEMBER 1995 DATI,Y MEAN VALUES

\begin{tabular}{|c|c|c|c|c|c|c|c|c|c|c|c|c|}
\hline DAY & $O C T$ & NoIV & $\mathrm{DEC}$ & JAN & EEB & MAR & APR & MAY & JUN & JUL & AUG & SEP \\
\hline 1 & .03 & .00 & e.05 & e. 01 & .07 & .00 & .01 & 1.2 & .84 & .10 & 1.4 & .07 \\
\hline 2 & .01 & .01 & e. 04 & e. 00 & .02 & .00 & .01 & 1.1 & .91 & .06 & .06 & .08 \\
\hline 3 & .01 & .02 & e. 03 & e. 00 & .02 & .01 & .01 & 1.7 & 2.1 & .04 & .05 & .07 \\
\hline 4 & .02 & .03 & e. 02 & e. 00 & .01 & $.0]$ & .01 & 1.3 & 1.6 & .04 & .05 & .06 \\
\hline 5 & .02 & .04 & e.02 & e. 00 & .01 & .01 & .00 & .59 & 1.0 & .04 & .04 & .03 \\
\hline 6 & .01 & .02 & e. 02 & e. 0 : & .01 & .02 & .00 & .42 & .36 & .04 & .03 & .03 \\
\hline 7 & .01 & .01 & e. 02 & e.0i & .02 & .01 & .00 & .32 & .30 & .09 & .02 & .03 \\
\hline 8 & .02 & .03 & e. 02 & e. 03 & .01 & .03 & .00 & .35 & 2.8 & .11 & .02 & .03 \\
\hline 9 & .00 & .06 & e. 02 & e.05 & .01 & .05 & .00 & .26 & 4.8 & .10 & .02 & .02 \\
\hline 10 & .01 & .04 & e. 02 & e. 04 & e. 01 & .02 & .03 & .21 & 1.2 & .07 & .02 & .02 \\
\hline 11 & .01 & .04 & e. 02 & e. 04 & e. 01 & .01 & .07 & .21 & .78 & .04 & .01 & .02 \\
\hline 12 & .01 & .04 & e.0? & e. 03 & e. $0 ?$ & .00 & .19 & .19 & .42 & .04 & .02 & .01 \\
\hline 13 & .01 & .04 & e. 01 & e. 04 & e. 04 & .00 & .06 & .12 & .30 & .11 & .01 & .01 \\
\hline 14 & .01 & .08 & e. 01 & e. 04 & e. 09 & .00 & .02 & .18 & .21 & .11 & .02 & .01 \\
\hline 15 & .02 & .18 & e. 00 & e.03 & e. 05 & .00 & .02 & .13 & .16 & .04 & .02 & .01 \\
\hline 16 & .02 & .06 & e. 00 & e. 03 & e.08 & .00 & .03 & .27 & .12 & .04 & .00 & .01 \\
\hline 11 & .07 & .05 & e.01 & e. 02 & e. 13 & .01 & .29 & e21 & .17 & .02 & .00 & .01 \\
\hline 18 & .02 & .02 & e.01 & e.01 & e.09 & e.01 & .44 & 2.0 & .20 & .02 & .00 & .02 \\
\hline 19 & .01 & .00 & e. 02 & e.01 & e.06 & e. 02 & 1.4 & .62 & .09 & .05 & .02 & .03 \\
\hline 20 & .01 & .02 & e. 02 & e. 02 & e. 03 & e. 02 & 1.2 & .68 & .06 & .03 & .01 & .04 \\
\hline 21 & .01 & .04 & e. 03 & e.01 & e. 01 & e. 01 & 1.8 & .47 & .07 & .02 & .01 & .05 \\
\hline 22 & .01 & e. 02 & e. 04 & e. 00 & e. 01 & .00 & 2.9 & .40 & .06 & .01 & .00 & .05 \\
\hline 23 & .02 & e. 02 & e. 03 & e. 01 & .01 & .00 & 2.5 & 1.7 & .07 & .01 & .00 & .02 \\
\hline 24 & .02 & e. 02 & e. 04 & e. 01 & .01 & .00 & 3.1 & 1.4 & .09 & .02 & .00 & .01 \\
\hline 25 & .01 & e. 02 & e. 05 & e. 02 & .01 & .00 & 2.2 & 1.1 & .06 & .02 & .00 & .01 \\
\hline 26 & .01 & e. 02 & e. 04 & e. 03 & .00 & .00 & 3.0 & 1.0 & .05 & .05 & .00 & .01 \\
\hline 27 & .00 & e. 02 & e. 04 & e. 04 & .00 & .00 & 4.1 & 2.2 & .05 & .08 & .00 & .01 \\
\hline 28 & .00 & e. 02 & e. 03 & e. 03 & .00 & .01 & 2.6 & 1.7 & .11 & .11 & .00 & .01 \\
\hline 29 & .00 & e. 50 & e. 02 & e. 04 & $\ldots$ & .01 & 3.6 & 1.4 & .42 & 2.2 & .00 & .01 \\
\hline 30 & .01 & e. 10 & e. 02 & e. 02 & $\cdots$ & .04 & 3.3 & 1.5 & .16 & 3.5 & e. 04 & .03 \\
\hline 31 & .00 & $\ldots$ & e. 01 & e. 08 & $\cdots$ & .03 & $\ldots$ & 1.5 & $\cdots$ & 3.5 & .07 & $\cdots$ \\
\hline TOTAL & 0.42 & 1.57 & 0.73 & 0.71 & 0.84 & 0.33 & 32.89 & 47.22 & 19.56 & 10.71 & 1.94 & 0.82 \\
\hline MEAN & .014 & .052 & .024 & .023 & .030 & .011 & 1.10 & 1.52 & .65 & .35 & .063 & .027 \\
\hline MAX & .07 & .50 & .05 & .08 & .13 & .05 & 4.1 & 21 & 4.8 & 3.5 & 1.4 & .08 \\
\hline MIN & .00 & .00 & .00 & .00 & .00 & .00 & .00 & .12 & .05 & .01 & .00 & .01 \\
\hline$A C-F T$ & .8 & 3.1 & 1.4 & 1.4 & 1.7 & .7 & 65 & 94 & 39 & 21 & 3.8 & 1.6 \\
\hline
\end{tabular}

STATISTICS OF MONTHLY MEAN DATA FOR WATER YEARS 1994 - 1995, BY WATER YEAR (WY)

\begin{tabular}{|c|c|c|c|c|c|c|c|c|c|c|c|c|}
\hline MBAN & .014 & .052 & .024 & .023 & .030 & .011 & .69 & .86 & .38 & .18 & .035 & .014 \\
\hline MAX & .014 & .052 & .024 & .023 & .030 & .011 & 1.10 & 1.52 & .65 & .35 & .063 & .027 \\
\hline (WY) & 399 & 1995 & 1995 & $: 995$ & 1995 & 1995 & 1995 & 1995 & 1995 & 1995 & 1995 & 1995 \\
\hline MIN & .014 & .052 & .024 & .023 & .030 & .011 & .29 & .19 & .11 & .014 & .007 & .000 \\
\hline$(W Y)$ & 1995 & 1995 & 1995 & 1995 & 1995 & 1995 & 1994 & 1994 & 1994 & 1994 & 1994 & 1994 \\
\hline
\end{tabular}

SUMMARY STATISTICS

ANNUAL TOTAL

ANNUAL MEAN

HIGHEST ANNUAL MEAN

LOWEST ANNUAL MEAN

HIGHEST DAILY MEAN

LOWEST DAILY MEAN

ANNUAL SEVEN-DAY MINIMUM

ANNUAL RUNOFF (AC-FT)

10 PERCENT EXCEEDS

50 PERCENT EXCEEDS

90 PERCENT EXCEEDS
FOR 1995 WATER YEAR

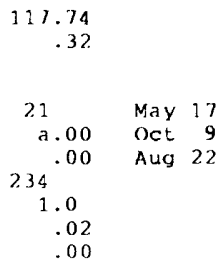

WATER YEARS 1994 - 1995

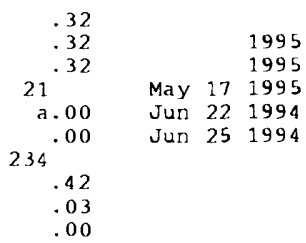

e Fstimated

a No flow many days 
SOUTH WOMAN CREEK AT WEST BUFFER ZONE FENCE LINE

SITE NUMBER. - - GSO6

STATION IDENTIFICATION. - 395253105131700

LOCATION. - Lat $39^{\circ} 52^{\prime} 53^{\prime \prime}$, long $105^{\circ} 13^{\prime} 17^{\prime \prime}$, in $\mathrm{SW}^{1} / 4 \mathrm{NW}^{1} / 4 \mathrm{sec} .15, \mathrm{~T} .2 \mathrm{~s} ., \mathrm{R} .70$ w. , Jefferson County, Hydrologic Unit 10190003 , Rocky Flats Environmental Technology Site, about 400 feet downstream from the west site fence line.

DRAINAGE AREA. $-0.28 \mathrm{mi}^{2}$.

PERIOD OF RECORD. - March 1994 to current year.

GAGE. - Water-stage recorder and Parshall flume. Elevation of gage is $6,063 \mathrm{ft}$ above sea level. REMARKS. - -Records fair.

DISCHARGE, CUBIC FEET PER SECOND, WATER YEAR OC SOBER 1993 TO SEPTEMBER 1994

\begin{tabular}{|c|c|c|c|c|c|c|c|c|c|c|c|c|}
\hline DAY & $\mathscr{N T}$ & NOV & DEC & JAN & FEB & MAR & $\mathrm{APR}$ & MAY & JUN & JUL & AUG & SEP \\
\hline 1 & $\cdots$ & $\cdots$ & $\cdots$ & $\cdots$ & $\cdots$ & $\cdots$ & .01 & .07 & .00 & .00 & .00 & .00 \\
\hline 2 & $\cdots$ & $\cdots$ & $\ldots$ & $\cdots$ & $\cdots$ & $\cdots$ & $0 ?$ & .08 & .00 & .01 & .00 & .00 \\
\hline 3 & $\cdots$ & $\cdots$ & $\cdots$ & $\cdots$ & $\cdots$ & $\cdots$ & .01 & .06 & .00 & .01 & .00 & .00 \\
\hline 4 & $\cdots$ & $\cdots$ & $\cdots$ & $\cdots$ & $\ldots$ & $\ldots$ & .01 & .05 & .00 & .01 & .00 & .00 \\
\hline 5 & $\cdots$ & $\cdots$ & $\cdots$ & $\cdots$ & $\cdots$ & $\cdots$ & .02 & .04 & .00 & .01 & .00 & .00 \\
\hline 6 & $\cdots$ & $\cdots$ & $\cdots$ & $\cdots$ & $\cdots$ & $\cdots$ & .02 & .03 & .00 & .01 & .00 & .00 \\
\hline 7 & $\cdots$ & $\cdots$ & $\cdots$ & $\cdots$ & $\cdots$ & $\cdots$ & .01 & .03 & .00 & .01 & .00 & .00 \\
\hline 8 & $\cdots$ & $\cdots$ & $\cdots$ & $\cdots$ & $\cdots$ & $\cdots$ & .01 & .02 & .00 & .01 & .00 & .00 \\
\hline 9 & $\cdots$ & $\ldots$ & $\ldots$ & $\ldots$ & $\cdots$ & $\cdots$ & .01 & .03 & .00 & .01 & .00 & .00 \\
\hline 10 & $\cdots$ & $\cdots$ & $\cdots$ & $\cdots$ & $\cdots$ & $\cdots$ & .02 & .03 & .00 & .00 & .01 & .00 \\
\hline 11 & $\cdots$ & $\cdots$ & $\cdots$ & $\cdots$ & $\cdots$ & $\cdots$ & .02 & .02 & .00 & .00 & .00 & .00 \\
\hline 12 & $\cdots$ & $\cdots$ & $\cdots$ & $\cdots$ & $\cdots$ & $\cdots$ & .10 & .02 & .02 & .00 & .00 & .00 \\
\hline 13 & $\cdots$ & $\cdots$ & $\cdots$ & $\cdots$ & $\cdots$ & $\cdots$ & .07 & .04 & .01 & .00 & .00 & .00 \\
\hline 14 & $\cdots$ & $\cdots$ & $\cdots$ & $\cdots$ & $\cdots$ & $\cdots$ & .04 & .03 & .01 & .00 & .00 & .00 \\
\hline 15 & $\cdots$ & $\cdots$ & $\cdots$ & $\cdots$ & $\cdots$ & $\cdots$ & .02 & .02 & .01 & .01 & .00 & .00 \\
\hline 16 & $\cdots$ & $\cdots$ & $\cdots$ & $\cdots$ & $\cdots$ & $\cdots$ & .01 & .02 & .01 & .01 & .00 & .00 \\
\hline 17 & $\cdots$ & $\cdots$ & $\cdots$ & $\cdots$ & $\cdots$ & $\cdots$ & .01 & .02 & .01 & .00 & .00 & .00 \\
\hline 18 & $\cdots$ & $\cdots$ & $\cdots$ & $\ldots$ & $\cdots$ & $\cdots$ & .01 & .02 & .01 & .00 & .00 & .00 \\
\hline 19 & $\cdots$ & $\cdots$ & $\ldots$ & $\ldots$ & $\cdots$ & $\cdots$ & .01 & .02 & .01 & .00 & .00 & .00 \\
\hline 20 & $\cdots$ & $\cdots$ & $\cdots$ & $\cdots$ & $\cdots$ & $\cdots$ & .00 & .01 & .01 & .00 & .00 & .00 \\
\hline 21 & $\cdots$ & $\cdots$ & $\cdots$ & $\cdots$ & $\cdots$ & $\cdots$ & .00 & .01 & .02 & .00 & .00 & .00 \\
\hline 22 & $\cdots$ & $\cdots$ & $\cdots$ & $\ldots$ & $\ldots$ & $\cdots$ & .00 & .01 & .02 & .00 & .00 & .00 \\
\hline 23 & $\cdots$ & $\cdots$ & $\cdots$ & $\cdots$ & $\cdots$ & $\cdots$ & .00 & .01 & .03 & .00 & .00 & .00 \\
\hline 24 & $\cdots$ & $\cdots$ & $\cdots$ & $\cdots$ & $\cdots$ & $\cdots$ & .00 & .01 & .01 & .00 & .00 & .00 \\
\hline 25 & $\cdots$ & $\cdots$ & $\cdots$ & $\cdots$ & $\cdots$ & $\cdots$ & .02 & .01 & .01 & .00 & .00 & .00 \\
\hline 26 & $\ldots$ & $\ldots$ & $\ldots$ & $\ldots$ & $\ldots$ & $\ldots$ & .02 & .01 & .01 & .00 & .00 & .00 \\
\hline 27 & $\cdots$ & $\cdots$ & $\cdots$ & $\cdots$ & $\cdots$ & $\cdots$ & .03 & .01 & .01 & .00 & .00 & .00 \\
\hline 28 & $\cdots$ & $\cdots$ & $\cdots$ & $\cdots$ & $\cdots$ & $\cdots$ & .04 & .01 & .01 & .00 & .00 & .00 \\
\hline 29 & $\cdots$ & $\cdots$ & $\ldots$ & $\cdots$ & $\cdots$ & $\ldots$ & .09 & .01 & .01 & .00 & .00 & .00 \\
\hline 30 & $\cdots$ & $\cdots$ & $\cdots$ & $\cdots$ & $\cdots$ & $\cdots$ & .11 & .00 & .01 & .00 & .00 & .00 \\
\hline 31 & $\cdots$ & $\cdots$ & $\cdots$ & $\cdots$ & $\cdots$ & .01 & $\cdots$ & .00 & $\cdots$ & .00 & .00 & $\cdots$ \\
\hline TOTAL & $\cdots$ & $\cdots$ & $\cdots$ & $\cdots$ & $\cdots$ & $\cdots$ & 0.73 & 0.75 & 0.24 & 0.10 & 0.01 & 0.00 \\
\hline MEAN & $\cdots$ & $\cdots$ & $\cdots$ & $\cdots$ & $\cdots$ & $\cdots$ & .024 & .024 & .008 & .003 & .000 & .000 \\
\hline MAX & $\cdots$ & $\cdots$ & $\cdots$ & $\cdots$ & $\cdots$ & $\cdots$ & .11 & .08 & .03 & .01 & .01 & .00 \\
\hline MIN & $\cdots$ & $\cdots$ & $\cdots$ & $\cdots$ & $\cdots$ & $\cdots$ & .00 & .00 & .00 & .00 & .00 & .00 \\
\hline$A C \cdot F T$ & $\cdots$ & $\cdots$ & $\cdots$ & $\cdots$ & $\cdots$ & $\cdots$ & 1.4 & 1.5 & .5 & .2 & .02 & .00 \\
\hline
\end{tabular}


SITE NUMBER. - GSO6

STATION IDENTIFICATION. - 395253105131700

LOCATION. - Lat $39^{\circ} 52^{\prime} 53^{\prime \prime}$, Iong $105^{\circ} 13^{\prime} 17^{\prime \prime}$, in SW $/ 4 \mathrm{NW}^{1} / 4$ sec. 15, T. 2 S., R. 70 w. , Jefferson County, Hydrologic Unit 10190003 , Rocky Flats Environmental Technology Site, about 400 feet downstream from the west site fence line.

DRAINAGE AREA. - $0.28 \mathrm{mi}^{2}$.

PERIOD OF RECORD. - -March 1994 to December 1994. March 1990 to current year.

GAGE. - Water-stage recorder and Parshall flume. Elevation of gage is 6,063 ft above sea level.

REMARKS.--Records fair except for estimated daily discharges, which are poor. Station discontinued December 22, 1994, to March 12, 1995.

DisCHARGe, CUBIC FEET PER SECOND, WATER YEAR (OCT(UBER 1994 TO SEPTEMBER 1995

\begin{tabular}{|c|c|c|c|c|c|c|c|c|c|c|c|c|}
\hline DAY & $\mathfrak{x}_{\mathrm{T}}$ & NOV & $\mathrm{OEC}$ & JAN & FEB & MAR & A PR & MAY & JUN & JUL & AUG & SEP \\
\hline 1 & .00 & .00 & e.00 & $\ldots$ & $\ldots$ & $\cdots$ & .00 & .24 & $\therefore 6$ & .05 & .03 & .00 \\
\hline 2. & .00 & .00 & e. 00 & $\cdots$ & $\ldots$ & $\cdots$ & .00 & .14 & .20 & .04 & .03 & .00 \\
\hline 3 & .00 & e.01 & e. 00 & $\cdots$ & $\cdots$ & $\cdots$ & .00 & .17 & .35 & .04 & .03 & .00 \\
\hline 4 & .00 & e.01 & e.00 & $\ldots$ & $\cdots$ & $\ldots$ & .00 & .15 & .35 & .04 & .03 & .00 \\
\hline 5 & .00 & $e .01$ & e.00 & $\cdots$ & $\cdots$ & $\cdots$ & .00 & .07 & .19 & .03 & .03 & .00 \\
\hline 6 & .00 & e.01 & e.00 & $\ldots$ & $\cdots$ & $\ldots$ & .00 & .05 & .12 & .03 & .03 & .00 \\
\hline 7 & .00 & e. 01 & e.00 & $\cdots$ & $\cdots$ & $\cdots$ & .00 & .03 & .20 & .03 & .02 & .00 \\
\hline 8 & .00 & $e .01$ & $e .00$ & $\cdots$ & $\ldots$ & $\cdots$ & .00 & .03 & .43 & .03 & .00 & .00 \\
\hline 9 & .00 & e.01 & e. 00 & $\cdots$ & $\cdots$ & $\cdots$ & .00 & .02 & .99 & .03 & .00 & .00 \\
\hline 10 & .00 & $e .01$ & e.00 & $\cdots$ & $\cdots$ & $\cdots$ & .02 & .02 & .23 & .03 & .00 & .00 \\
\hline 11 & .00 & $e .01$ & e.00 & $\ldots$ & $\ldots$ & $\ldots$ & .00 & .02 & .14 & .03 & .00 & .00 \\
\hline 12 & .00 & $e .01$ & e.00 & $\cdots$ & $\ldots$ & $\ldots$ & .01 & .02 & .10 & .03 & .00 & .00 \\
\hline 13 & .00 & $e .01$ & $e .00$ & $\ldots$ & $\ldots$ & .00 & .00 & .01 & .08 & .04 & .00 & .00 \\
\hline 14 & .00 & e.01 & e. 00 & $\cdots$ & $\cdots$ & .00 & .00 & .01 & .07 & .05 & .00 & .00 \\
\hline 15 & .01 & .00 & e. 00 & $\cdots$ & $\cdots$ & .00 & .00 & .01 & .06 & .05 & .00 & .00 \\
\hline 16 & e. 01 & .00 & e.00 & $\ldots$ & $\ldots$ & .00 & .00 & .02 & .05 & .05 & .00 & .00 \\
\hline 17 & .00 & e.01 & e.00 & $\ldots$ & $\ldots$ & .00 & .01 & $\mathrm{e} 10^{.02}$ & .06 & .05 & .00 & .00 \\
\hline 18 & .00 & e.01 & $e .00$ & $\ldots$ & $\ldots$ & .00 & .02 & .29 & .05 & .06 & .01 & .00 \\
\hline 19 & .00 & e. 00 & e.00 & $\cdots$ & $\cdots$ & .00 & .13 & .11 & .04 & .07 & .01 & .00 \\
\hline 20 & .00 & $e .00$ & e. 00 & $\cdots$ & $\cdots$ & .00 & .06 & .07 & .03 & .06 & .01 & .00 \\
\hline 21 & .00 & e. 00 & e. 00 & $\ldots$ & $\ldots$ & .00 & .14 & .05 & .03 & .05 & .00 & .00 \\
\hline 22 & .00 & e.00 & $\ldots$ & $\cdots$ & $\cdots$ & .00 & .28 & .05 & .03 & .04 & .00 & .00 \\
\hline 23 & .00 & e. 00 & $\ldots$ & $\ldots$ & $\ldots$ & .00 & .27 & .27 & .03 & .04 & .00 & .00 \\
\hline 24 & .00 & e.00 & $\ldots$ & $\ldots$ & $\ldots$ & .00 & .46 & .56 & .04 & .04 & .00 & .00 \\
\hline 25 & .00 & e.00 & $\cdots$ & $\cdots$ & $\cdots$ & .00 & .24 & .29 & .03 & .03 & .00 & .00 \\
\hline 26 & .00 & e. 00 & $\ldots$ & $\ldots$ & $\cdots$ & .00 & .71 & .28 & .03 & .03 & .00 & .00 \\
\hline 27 & .00 & e. 00 & $\cdots$ & $\cdots$ & $\cdots$ & .00 & .82 & e. 99 & .02 & .03 & .00 & .00 \\
\hline 28 & .00 & e. 00 & $\cdots$ & $\ldots$ & $\cdots$ & .00 & .37 & e. 64 & .05 & .03 & .00 & .00 \\
\hline 29 & .00 & e. 00 & $\cdots$ & $\ldots$ & $\cdots$ & .00 & .75 & .74 & .10 & .03 & .00 & .00 \\
\hline 30 & .00 & e.00 & $\ldots$ & $\ldots$ & $\ldots$ & .02 & .88 & .47 & .05 & .03 & .00 & .00 \\
\hline 31 & .00 & 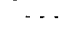 & $\cdots$ & $\ldots$ & $\ldots$ & .13 & 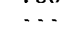 & .26 & $\cdots$ & .04 & .00 & .00 \\
\hline TOTAL & 0.02 & 0.14 & $\ldots$ & $\ldots$ & . & $\ldots$ & 5.17 & 16.08 & 4.21 & 1.23 & 0.23 & 0.00 \\
\hline MEAN & .001 & .005 & $\ldots$ & $\ldots$ & $\ldots$ & $\ldots$ & .17 & .52 & .14 & .040 & .007 & .000 \\
\hline $\operatorname{MAX}$ & .01 & .01 & $\cdots$ & $\cdots$ & $\cdots$ & $\cdots$ & .88 & 10 & .99 & .07 & .03 & .00 \\
\hline MIN & .00 & .00 & $\ldots$ & $\cdots$ & $\cdots$ & $\cdots$ & .00 & .01 & .02 & .03 & .00 & .00 \\
\hline $\mathrm{AC}-\mathrm{FT}$ & .04 & .3 & $\ldots$ & $\ldots$ & $\ldots$ & $\ldots$ & 10 & 32 & 8.4 & 2.4 & .5 & .00 \\
\hline
\end{tabular}

STATISTICS OF MONTHLY MEAN DATA FOR WATER YEARS 1994 - 1995, BY WATER YEAR (WY)

\begin{tabular}{|c|c|c|c|c|c|c|c|c|c|c|c|c|}
\hline MEAN & .001 & .005 & $\cdots$ & $\cdots$ & $\cdots$ & $\cdots$ & .098 & .27 & .074 & .021 & .004 & .000 \\
\hline $\operatorname{MAX}$ & .001 & .003 & $\cdots$ & $\cdots$ & $\cdots$ & $\cdots$ & .17 & .52 &.$: 4$ & .040 & .007 & .000 \\
\hline (WY) & 1995 & 1995 & $\cdots$ & $\cdots$ & $\cdots$ & $\cdots$ & 1995 & 1995 & i995 & 1995 & 1995 & 1994 \\
\hline MIN & .001 & .005 & $\cdots$ & $\cdots$ & $\cdots$ & $\cdots$ & .024 & .024 & .008 & .003 & .000 & .000 \\
\hline (WY) & 1995 & 1995 & $\cdots$ & $\ldots$ & $\cdots$ & $\cdots$ & 1994 & 1994 & 1994 & 1994 & 1994 & 1994 \\
\hline
\end{tabular}

SUMMARY STATISTICS

HIGHFST DALLY MEAN LOWEST DAILY MEAN

ANNUAL SEVFN-DAY MINIMUM

10 PERCENT EXCEEDS

,O PERCENT EXCEEDS

90 PERCENT EXCEEDS

a No flow many days

e Estimated
WATER YEARS $1994-1995$

$\begin{array}{rll}10 & \text { May } 17 & 1995 \\ \text { a.00 } & \text { Apr } 20 & 1994 \\ .00 & \text { May } 30 & 1994 \\ .07 & & \end{array}$

0
00
00
00
00
00
00
00
00
00
00
00
00
00
00
00
00
00
00
00
00
00
00
00
00
00
00
00
00
00
00
00
00
00
00
00
0

\section{0}


SOUTH WALNUT CREEK BELOW POND B-4

SITE NUMBER. - - GSO9

STATION IDENTIFICATION. - - 395342105110800

LOCATION, - Lat $39^{\circ} 53^{\prime} 42^{\prime \prime}$, long $105^{\circ} 11^{\prime} 08^{\prime \prime}$, in $\mathrm{SE}^{1} / 4 \mathrm{NE}^{1} / 4 \mathrm{sec} .11, \mathrm{~T} .2 \mathrm{~S}$., R. 70 w., Jefferson County, Hydrologic Unit 10190003, Rocky Flats Environmental Technology site, just downstream from Pond B- 4 dam.

DRAINAGE AREA. - - $0.33 \mathrm{mi}^{?}$.

PERIOD OF RECORD. - March 1994 to current year.

GAGE. - Water-stage recorder and rectangular weir. Elevation of gage is $5,820 \mathrm{ft}$ above sea level.

REMARKS. - Records fair except for estimated daily discharges, which are poor. Flow is regulated by $B$-Series ponds.

DISCHARGE, CUBIC FEET PER SECUND, WATER YEAR (KCTOBER 1993 TO SEPTEMBER 1994

\begin{tabular}{|c|c|c|c|c|c|c|c|c|c|c|c|c|}
\hline DAY & $(x+T$ & NOV & DEC & JAN & FEB & MAR & APR & MAY & JUN & JUI, & AUG & SEP \\
\hline 1 & $\cdots$ & $\cdots$ & $\cdots$ & $\ldots$ & $\cdots$ & $\cdots$ & .36 & .58 & .35 & .17 & .16 & .15 \\
\hline 2 & $\cdots$ & $\ldots$ & $\ldots$ & $\cdots$ & $\ldots$ & $\ldots$ & .55 & .61 & .40 & .01 & .02 & .29 \\
\hline 3 & $\cdots$ & $\cdots$ & $\ldots$ & $\cdots$ & $\cdots$ & $\cdots$ & .29 & .41 & .69 & .21 & .26 & .11 \\
\hline 4 & $\cdots$ & $\ldots$ & $\cdots$ & $\cdots$ & $\cdots$ & $\ldots$ & .30 & .45 & .06 & .12 & .37 & .14 \\
\hline 3 & $\cdots$ & $\ldots$ & $\cdots$ & $\cdots$ & $\cdots$ & $\cdots$ & .52 & .59 & .21 & .30 & .04 & .15 \\
\hline 6 & $\cdots$ & $\cdots$ & $\cdots$ & $\ldots$ & $\cdots$ & $\cdots$ & .38 & .55 & .29 & .23 & .06 & .31 \\
\hline 7 & $\cdots$ & $\ldots$ & $\ldots$ & $\ldots$ & $\cdots$ & $\cdots$ & .51 & .20 & .40 & .00 & .06 & .22 \\
\hline 8 & $\cdots$ & $\cdots$ & $\ldots$ & $\ldots$ & $\ldots$ & $\cdots$ & .34 & .14 & .25 & .28 & .17 & .50 \\
\hline 9 & $\cdots$ & $\cdots$ & $\cdots$ & $\cdots$ & $\cdots$ & $\cdots$ & .58 & .30 & .15 & .14 & .20 & .10 \\
\hline 10 & $\cdots$ & $\cdots$ & $\cdots$ & $\cdots$ & $\cdots$ & $\cdots$ & 1.2 & .50 & .23 & .12 & .94 & .09 \\
\hline 11 & $\cdots$ & $\cdots$ & $\cdots$ & $\cdots$ & $\cdots$ & $\cdots$ & .70 & .39 & .13 & .21 & 1.1 & .07 \\
\hline 12 & $\cdots$ & $\cdots$ & $\cdots$ & $\cdots$ & $\cdots$ & $\cdots$ & 1.0 & .32 & .16 & .15 & .45 & .39 \\
\hline 13 & $\cdots$ & $\cdots$ & $\cdots$ & $\cdots$ & $\cdots$ & $\cdots$ & .49 & e1.8 & .24 & .25 & .06 & .08 \\
\hline 14 & $\ldots$ & $\cdots$ & $\cdots$ & $\ldots$ & $\ldots$ & $\cdots$ & .47 & e. 40 & .22 & .41 & .15 & .19 \\
\hline 15 & $\cdots$ & $\cdots$ & $\cdots$ & $\cdots$ & $\cdots$ & $\cdots$ & .45 & e. 36 & .11 & .02 & .17 & .19 \\
\hline 16 & $\cdots$ & $\cdots$ & $\cdots$ & $\cdots$ & $\cdots$ & .29 & .41 & e. 36 & .41 & .09 & .18 & .48 \\
\hline 17 & $\cdots$ & $\cdots$ & $\cdots$ & $\cdots$ & $\cdots$ & .52 & .35 & .31 & .14 & .10 & .30 & .13 \\
\hline 18 & $\cdots$ & $\cdots$ & $\ldots$ & $\cdots$ & $\ldots$ & .23 & .37 & .45 & .07 & .20 & .29 & .11 \\
\hline 19 & $\cdots$ & $\cdots$ & $\cdots$ & $\cdots$ & $\cdots$ & .28 & .45 & .39 & .15 & .20 & .06 & .21 \\
\hline 20 & $\cdots$ & $\cdots$ & $\cdots$ & $\cdots$ & $\cdots$ & .24 & .13 & .60 & .24 & .21 & .24 & .22 \\
\hline 21 & $\cdots$ & $\cdots$ & $\cdots$ & $\cdots$ & $\cdots$ & .23 & .64 & .36 & .39 & .18 & .20 & .40 \\
\hline 22 & $\cdots$ & $\cdots$ & $\cdots$ & $\cdots$ & $\cdots$ & .08 & .19 & .34 & .36 & .19 & e. 20 & .45 \\
\hline 23 & $\cdots$ & $\cdots$ & $\cdots$ & $\cdots$ & $\cdots$ & .28 & .20 & .53 & .25 & .19 & e. 20 & .23 \\
\hline 24 & $\cdots$ & $\cdots$ & $\cdots$ & $\cdots$ & $\cdots$ & .48 & .27 & .57 & .23 & .18 & .15 & .20 \\
\hline 25 & $\cdots$ & $\ldots$ & $\cdots$ & $\cdots$ & $\ldots$ & .23 & 1.5 & .28 & .16 & .18 & .26 & .37 \\
\hline 26 & $\cdots$ & $\cdots$ & $\cdots$ & $\cdots$ & $\cdots$ & .25 & .45 & .45 & .16 & .20 & .17 & .34 \\
\hline 27 & $\cdots$ & $\cdots$ & $\cdots$ & $\cdots$ & $\cdots$ & .42 & .60 & .19 & .15 & .05 & .10 & .40 \\
\hline 28 & $\cdots$ & $\cdots$ & $\cdots$ & $\cdots$ & $\cdots$ & .38 & .46 & .21 & .08 & .22 & .21 & .30 \\
\hline 29 & $\cdots$ & $\cdots$ & $\cdots$ & $\cdots$ & $\cdots$ & .81 & 1.3 & .08 & .18 & .22 & .17 & .13 \\
\hline 30 & $\cdots$ & $\ldots$ & $\cdots$ & $\cdots$ & $\cdots$ & .53 & .66 & .22 & .21 & .23 & .12 & .18 \\
\hline 31 & $\cdots$ & $\cdots$ & $\cdots$ & $\cdots$ & $\cdots$ & .34 & $\cdots$ & .58 & $\cdots$ & .10 & .46 & \\
\hline TOTAL & $\cdots$ & $\cdots$ & $\cdots$ & $\cdots$ & $\cdots$ & $\cdots$ & 16.12 & 13.52 & 7.07 & 5.36 & 7.52 & 7.13 \\
\hline MEAN & $\cdots$ & $\cdots$ & $\cdots$ & $\cdots$ & $\ldots$ & $\cdots$ & .54 & .44 & .24 & .17 & .24 & .24 \\
\hline $\operatorname{MAX}$ & $\cdots$ & $\cdots$ & $\cdots$ & $\cdots$ & $\ldots$ & $\cdots$ & 1.5 & 1.8 & .69 & .41 & 1.1 & .50 \\
\hline MIN & $\cdots$ & $\cdots$ & $\cdots$ & $\cdots$ & $\cdots$ & $\cdots$ & .13 & .08 & .05 & .00 & .02 & .07 \\
\hline$A C \cdot F^{T}$ & $\cdots$ & $\cdots$ & $\cdots$ & $\cdots$ & $\cdots$ & $\cdots$ & 32 & 27 & 14 & 11 & 15 & 14 \\
\hline
\end{tabular}

e Estimatert 
SITE NUMBER. - - GS09

STATION IDENTIFICATION. - - 395342105110800

LOCATION. - Lat $39^{\circ} 53^{\prime} 42^{\prime \prime}$, long $105^{\circ} 11^{\prime} 08^{\prime \prime}$, in $\mathrm{SE}^{1} / 4 \mathrm{NE}^{1} / 4$ sec. 11, T. $2 \mathrm{~S}$. , R. 70 W. , Jefferson County, Hydrologic Unit 10190003, Rocky Flats Environmental Technology Site, just downstream from Pond B-4 dam.

DRAINAGE AREA. $-0.33 \mathrm{mi}^{2}$.

PERIOD OF RECORD. - March 1994 to current year.

GAGE.- Water-stage recorder and rectangular weir. Elevation of gage is $5,820 \mathrm{ft}$ above sea level.

REMARKS. - - Records fair except for estimated daily discharges, which are poor. Flow is regulated by B-Series ponds.

DISCHARGE, CUBIC FEET PER SECUND, WATER YEAR OCTUBER Z 994 TO SEPTEMBER 1995

\begin{tabular}{|c|c|c|c|c|c|c|c|c|c|c|c|c|}
\hline DAY & ()$_{T}$ & $\mathrm{NOIV}$ & DEC & JAN & FEB & MAR & $A P R$ & MAY & JUN & JUL, & AUG & SEP \\
\hline 1 & .23 & .49 & e. 20 & .07 & .18 & .20 & e. 09 & .87 & .66 & .50 & .33 & .34 \\
\hline 2 & .32 & .17 & .08 & .12 & .29 & .13 & .16 & .50 & .65 & .27 & .15 & .08 \\
\hline 3 & .41 & .21 & .09 & .19 & .10 & .27 & .40 & .94 & 1.2 & .90 & .31 & .19 \\
\hline 4 & .10 & .33 & .09 & .21 & .02 & .21 & .04 & .69 & .66 & .22 & .17 & .17 \\
\hline 5 & .28 & .21 & .16 & .13 & .16 & .23 & .20 & .26 & .75 & .47 & .11 & .67 \\
\hline 6 & .23 & e. 21 & .20 & .08 & .36 & .52 & .14 & e. 37 & $.4 \mathrm{~J}$ & .20 & .27 & .32 \\
\hline 7 & .37 & .41 & .30 & .01 & .07 & .08 & .13 & .09 & .44 & .49 & .32 & .46 \\
\hline 8 & .09 & .61 & .16 & .09 & .18 & .23 & .17 & .66 & 1.6 & .12 & .21 & .22 \\
\hline 9 & .20 & .30 & .21 & .11 & .21 & .28 & .26 & .47 & 2.6 & .10 & .40 & .11 \\
\hline 10 & .18 & .31 & .05 & .24 & .04 & .21 & .48 & e. 19 & .77 & .42 & .15 & .21 \\
\hline 11 & .19 & .26 & .00 & .17 & $\therefore 8$ & .27 & .50 & e. 10 & .61 & .38 & .05 & .17 \\
\hline 12 & .26 & e. 25 & .14 & .17 & $\therefore 8$ & .24 & .52 & .18 & .80 & .30 & .24 & .19 \\
\hline 13 & .39 & .24 & .20 & .17 & .14 & .39 & .26 & e. 20 & .66 & .59 & .10 & .34 \\
\hline 14 & .24 & .63 & .13 & .11 & .13 & .25 & .26 & .26 & .29 & .09 & .33 & .06 \\
\hline 15 & .26 & e. 18 & .15 & .21 & .36 & .19 & .28 & e. 44 & .81 & .11 & .13 & .28 \\
\hline 16 & .07 & .18 & .12 & .14 & .22 & .13 & .35 & 1.1 & .37 & .43 & .27 & .23 \\
\hline 17 & .63 & .24 & .21 & .20 & $\therefore 4$ & .22 & 1.2 & e14 & .44 & .20 & .17 & .11 \\
\hline 18 & .28 & .34 & e. 15 & .13 & .26 & .18 & 1.4 & .82 & .43 & .50 & .40 & .57 \\
\hline 19 & .36 & .10 & .09 & .21 & .08 & .36 & 1.9 & 1.0 & .81 & .57 & .20 & .23 \\
\hline 20 & .35 & .05 & .26 & .11 & .35 & .19 & .87 & .29 & .19 & .31 & .03 & .45 \\
\hline 21 & .25 & .21 & .10 & .09 & .23 & e. 17 & 2.1 & .24 & .60 & .32 & .37 & .65 \\
\hline 22 & .24 & .19 & .07 & .04 & .26 & .22 & .78 & .64 & .17 & .34 & .39 & .30 \\
\hline 23 & .19 & .19 & .12 & .14 & .35 & e. 17 & .55 & 1.7 & .74 & .24 & .31 & .41 \\
\hline 24 & .25 & .13 & .09 & .16 & .13 & e. 24 & .78 & 1.5 & .25 & .03 & .42 & .48 \\
\hline 25 & .24 & .13 & .05 & .35 & .11 & .01 & 1.4 & .57 & .17 & .78 & .21 & .19 \\
\hline 26 & e. 24 & .07 & .16 & .14 & .09 & .12 & 3.1 & 1.1 & .59 & .22 & .07 & .35 \\
\hline 27 & e. 24 & .09 & .27 & .29 & .33 & e. 18 & .83 & 2.1 & .09 & .03 & .49 & .40 \\
\hline 28 & .18 & .11 & .03 & .01 & .31 & .35 & 1.3 & 2.5 & 1.4 & .24 & .20 & .26 \\
\hline 29 & .17 & .22 & .03 & .10 & $\cdots$ & .27 & 1.9 & .89 & 2.7 & .16 & .46 & .17 \\
\hline 30 & .14 & e. 20 & .00 & .15 & $\cdots$ & e. 34 & 1.6 & .88 & .26 & .26 & .32 & .36 \\
\hline 31 & .21 & $\ldots$ & .00 & .21 & $\cdots$ & e. 25 & $\cdots$ & 1.2 & $\cdots$ & .19 & .18 & $\cdots$ \\
\hline TOTAL & 7.79 & 7.26 & 3.91 & 4.55 & 5.46 & 7.10 & 23.95 & 36.75 & 22.16 & 9.98 & 8.36 & 8.97 \\
\hline MEAN & .25 & .2 .4 & .33 & .15 & .19 & .23 & .80 & 1.19 & .74 & .32 & .27 & .30 \\
\hline $\operatorname{MAX}$ & .63 & .63 & .30 & .35 & .36 & .52 & 3.1 & 14 & 2.7 & .90 & .75 & .67 \\
\hline MIN & .07 & .05 & .00 & .01 & .02 & .01 & .04 & .09 & .09 & .03 & .03 & .06 \\
\hline$A C-F T$ & 15 & 14 & 7.8 & 9.0 & 11 & 14 & 48 & 73 & 44 & 20 & 17 & 18 \\
\hline
\end{tabular}

STATISTICS OF MONTHLY MEAN DATA FOR WATER YEAKS 1994 - 1995, BY WATER YEAR (WY)

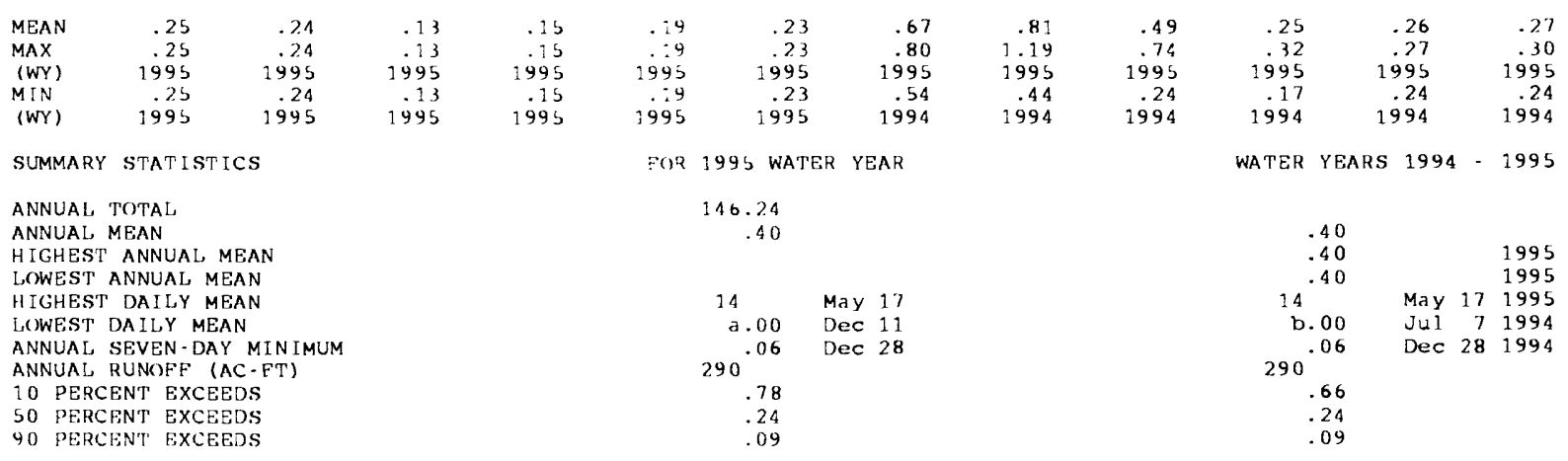

a also occurted Dec. 30-31

b No flow at times most years

e Estimated 
SOUTH WALNUT CREEK ABOVE B-SERIES BYFASS

SITE NUMBER. - - GS10

STATION IDENTIFICATION. - 395335105112700

LOCATION. - Lat $39^{\circ} 53^{\prime} 35^{\prime \prime}$, long $105^{\circ} 11^{\prime} 27^{\prime \prime}$, in $\mathrm{SW}^{1} / 4 \mathrm{NE}^{1} / 4$ sec.11, T. 2 S., R. 70 W., Jefferson County, Hydrologic Unit 10190003, Rocky Flats Environmental Technology site, just upstream from the B-1 Bypass above pond B-1.

DRAINAGE AREA. - $0.28 \mathrm{mi}^{2}$.

PERIOD OF RECORD. - March 1994 to current year.

GAGE. - Water-stage recorder and Parshall flume. Elevation of gage is $5,882 \mathrm{ft}$ above sea level. REMARKS.--Records poor.

DISCHARGE, CUBIC FEET PER SECOND, WATER YEAR OCTOBER 1993 TO SEPTEMBER 1994 DAILY MEAN VALUES

\begin{tabular}{|c|c|c|c|c|c|c|c|c|c|c|c|c|}
\hline DAY & ('CT & NoV & DEC & JAN & PEB & MAR & APR & MAY & JUN & JUL & AUG & SEP \\
\hline 1 & $\cdots$ & $\cdots$ & . . & $\ldots$ & $\cdots$ & $\cdots$ & .10 & .17 & .11 & .03 & .06 & .05 \\
\hline 2 & $\cdots$ & $\ldots$ & $\ldots$ & $\ldots$ & $\cdots$ & $\cdots$ & .12 & .27 & .10 & .03 & .06 & .03 \\
\hline 3 & $\cdots$ & $\cdots$ & $\cdots$ & $\cdots$ & $\cdots$ & $\cdots$ & .09 & .14 & .51 & .03 & .09 & .02 \\
\hline 4 & $\cdots$ & $\cdots$ & $\cdots$ & $\cdots$ & $\ldots$ & $\cdots$ & .10 & .13 & .11 & .03 & .07 & .02 \\
\hline 5 & $\cdots$ & $\cdots$ & $\cdots$ & $\cdots$ & $\cdots$ & $\cdots$ & .21 & .12 & .08 & .04 & .07 & .02 \\
\hline 6 & $\cdots$ & $\cdots$ & $\ldots$ & $\ldots$ & $\cdots$ & $\ldots$ & .13 & .13 & .08 & .03 & .08 & .04 \\
\hline 7 & $\cdots$ & $\cdots$ & $\ldots$ & $\cdots$ & $\ldots$ & $\cdots$ & .09 & .11 & .07 & .03 & .13 & .05 \\
\hline 8 & $\cdots$ & $\cdots$ & $\cdots$ & $\cdots$ & $\cdots$ & $\cdots$ & .07 & .10 & .07 & .03 & .15 & .05 \\
\hline 9 & $\cdots$ & $\cdots$ & $\cdots$ & $\cdots$ & $\cdots$ & $\cdots$ & .52 & .15 & .07 & .03 & .15 & .06 \\
\hline 10 & $\cdots$ & $\cdots$ & $\cdots$ & $\cdots$ & $\cdots$ & $\cdots$ & 1.2 & .12 & .07 & .03 & .54 & .07 \\
\hline 11 & $\cdots$ & $\cdots$ & $\cdots$ & $\cdots$ & $\cdots$ & St.05 & .25 & .12 & .06 & .04 & .13 & .04 \\
\hline 12 & $\cdots$ & $\cdots$ & $\cdots$ & $\cdots$ & $\cdots$ & .07 & .47 & .11 & .06 & .04 & .11 & .04 \\
\hline 13 & $\cdots$ & $\cdots$ & $\cdots$ & $\cdots$ & $\cdots$ & .08 & .19 & .65 & .06 & .04 & .09 & .05 \\
\hline 14 & $\cdots$ & $\cdots$ & $\cdots$ & $\cdots$ & $\cdots$ & .06 & .13 & .13 & .06 & .04 & .08 & .05 \\
\hline 15 & $\cdots$ & $\cdots$ & $\cdots$ & $\cdots$ & $\cdots$ & .07 & .11 & .11 & .06 & .05 & .10 & .05 \\
\hline 16 & $\cdots$ & $\cdots$ & $\cdots$ & - . & $\cdots$ & .06 & .10 & .11 & .06 & .04 & .11 & .05 \\
\hline 17 & $\cdots$ & $\cdots$ & $\cdots$ & $\cdots$ & $\cdots$ & .06 & .10 & .10 & .07 & .03 & .11 & .03 \\
\hline 18 & $\cdots$ & $\cdots$ & $\cdots$ & $\cdots$ & $\cdots$ & .06 & .09 & .12 & .06 & .06 & .11 & .04 \\
\hline 19 & $\cdots$ & $\cdots$ & $\cdots$ & $\cdots$ & $\cdots$ & .05 & .09 & .10 & .06 & .05 & .10 & .04 \\
\hline 20 & $\cdots$ & $\cdots$ & $\cdots$ & $\cdots$ & $\cdots$ & .06 & .08 & .10 & .07 & .05 & .08 & .06 \\
\hline 21 & $\cdots$ & $\cdots$ & $\cdots$ & $\cdots$ & $\cdots$ & .08 & .08 & .09 & .23 & .04 & .08 & .31 \\
\hline 22 & $\cdots$ & $\cdots$ & $\cdots$ & $\cdots$ & $\cdots$ & .04 & .08 & .09 & .18 & .03 & .05 & .14 \\
\hline 23 & $\cdots$ & $\cdots$ & $\cdots$ & - . & $\cdots$ & .05 & .08 & .09 & .07 & .05 & .03 & .06 \\
\hline 24 & $\cdots$ & $\cdots$ & $\cdots$ & $\ldots$ & $\cdots$ & .06 & .08 & .10 & .05 & .04 & .02 & .06 \\
\hline $2 b$ & $\cdots$ & $\cdots$ & $\cdots$ & $\cdots$ & $\cdots$ & .05 & 1.1 & .10 & .04 & .04 & .03 & .06 \\
\hline 26 & $\cdots$ & $\cdots$ & $\cdots$ & $\cdots$ & $\cdots$ & .07 & .23 & .08 & .04 & .04 & .02 & .07 \\
\hline 27 & $\cdots$ & $\cdots$ & $\cdots$ & $\cdots$ & $\cdots$ & .18 & .23 & .08 & .04 & .05 & .01 & .08 \\
\hline 28 & $\cdots$ & $\cdots$ & $\cdots$ & $\cdots$ & $\cdots$ & .18 & .20 & .22 & .04 & .05 & .05 & .10 \\
\hline 29 & $\cdots$ & $\cdots$ & $\cdots$ & $\cdots$ & $\cdots$ & .56 & 1.1 & .07 & .04 & .05 & .02 & .09 \\
\hline 30 & $\cdots$ & $\cdots$ & $\cdots$ & $\cdots$ & $\cdots$ & .28 & .43 & .06 & .03 & .05 & .00 & .10 \\
\hline $3 ?$ & $\cdots$ & $\cdots$ & $\cdots$ & $\cdots$ & $\cdots$ & .12 & $\cdots$ & .35 & $\cdots$ & .06 & .06 & $\cdots$ \\
\hline TOTAL & $\cdots$ & $\ldots$ & $\cdots$ & $\ldots$ & $\cdots$ & $\ldots$ & 7.85 & 4.42 & 2.65 & 1.25 & 2.79 & 1.93 \\
\hline MEAN & $\cdots$ & $\cdots$ & $\cdots$ & $\cdots$ & - . & - . & .26 & .14 & .088 & .040 & .090 & .064 \\
\hline MAX & $\cdots$ & $\cdots$ & $\cdots$ & $\cdots$ & $\cdots$ & $\cdots$ & 1.2 & .65 & .51 & .06 & .54 & .31 \\
\hline MIN & $\cdots$ & $\cdots$ & $\cdots$ & $\cdots$ & $\cdots$ & $\cdots$ & .07 & .06 & .03 & .03 & .00 & .02 \\
\hline$A C-F T$ & $\cdots$ & $\cdots$ & $\cdots$ & $\cdots$ & $\cdots$ & $\cdots$ & 16 & 8.8 & 5.3 & 2.5 & 5.5 & 3.8 \\
\hline
\end{tabular}


SITE NUMBER. - - GS10

STATION IDENTIFICATION. - - 395335105112700

LOCATION. - Lat $39^{\circ} 53^{\prime} 35^{\prime \prime}$, long $105^{\circ} 11^{\prime} 27^{\prime \prime}$, in $\mathrm{SW}^{1} / 4 \mathrm{NE}^{1} / 4 \mathrm{sec} .11, \mathrm{~T} .2 \mathrm{~s} .$, R. 70 w., Jefferson County, Hydrologic Unit 10190003, Rocky Flats Environmental Technology Site, just upstream from the B1 Bypass above Pond B-1.

DRAINAGE AREA. - - $0.28 \mathrm{mi}^{2}$.

PERIOD OF RECORD. - March 1994 to current year.

GAGE.- Water-stage recorder and Parshall flume. Elevation of gage is 5,882 ft above sea level. REMARKS. - Records poor.

DISCHARGE, CUBIC PEET PER SECUND, WATER YEAR (CTUBER 1994 TU SEPTEMBER 1995

\begin{tabular}{|c|c|c|c|c|c|c|c|c|c|c|c|c|}
\hline DAY & $(x]^{\prime}$ & Nov & DEC & JAN & $\mathrm{FFB}$ & MAR & $A P R$ & MAY & JUN & JUL & AUG & SEP \\
\hline 1 & .20 & .04 & .06 & .00 & .06 & .08 & .07 & .32 & e. 34 & e. $2 b$ & .06 & .02 \\
\hline 2 & .10 & .05 & .05 & .00 & .04 & .06 & .07 & .39 & e. 78 & e. 23 & .05 & .02 \\
\hline 3 & .12 & .10 & .05 & .00 & .03 & .07 & .07 & .66 & e. 77 & e. 22 & .04 & .02 \\
\hline 4 & .11 & .26 & .05 & .00 & .03 & $.0 \%$ & .06 & .38 & e. 72 & e. 21 & .05 & .02 \\
\hline 5 & .10 & .14 & .05 & .00 & .03 & .07 & .06 & .18 & .41 & .16 & .05 & .03 \\
\hline 6 & .09 & .07 & .06 & .00 & .03 & .38 & .06 & .16 & .32 & .16 & .04 & .03 \\
\hline 7 & .04 & .06 & .05 & .00 & .02 & .12 & .06 & .15 & .28 & .15 & .05 & .03 \\
\hline 8 & .04 & .43 & e. 06 & .00 & .03 & .14 & .06 & .18 & 1.4 & .14 & .06 & .03 \\
\hline 9 & .03 & .14 & e. 05 & .00 & .03 & .09 & .10 & .13 & e 1.8 & .14 & .06 & .10 \\
\hline 10 & .03 & .07 & e. 05 & .00 & .02 & .08 & .58 & .13 & e. 40 & .14 & .04 & .05 \\
\hline 11 & .05 & .05 & .03 & .00 & .00 & .07 & .50 & .13 & e. 35 & .14 & .03 & .03 \\
\hline 12 & .04 & .04 & e. 03 & .00 & .00 & .07 & .41 & .15 & e. 36 & .14 & .02 & .03 \\
\hline 13 & .05 & .05 & .03 & .02 & .00 & .07 & .12 & .15 & e. 32 & .21 & .02 & .04 \\
\hline 14 & .05 & .45 & .03 & .03 & .63 & .07 & .09 & .12 & e. 36 & .32 & .03 & .04 \\
\hline 15 & .08 & .09 & e. 02 & .03 & .19 & .07 & .08 & .15 & e. 35 & .24 & .02 & .04 \\
\hline 16 & .08 & .08 & .04 & .02 & .13 & .10 & e. 37 & e. 77 & e. 33 & .24 & .02 & .04 \\
\hline 17 & .61 & .06 & .04 & .03 & .18 & .10 & 1.1 & e11 & e. 57 & .26 & .04 & .04 \\
\hline 18 & .06 & .06 & .04 & .02 & .10 & .08 & e1.2 & e. 27 & e. 37 & .23 & .29 & .30 \\
\hline 19 & .05 & .05 & .05 & .02 & .09 & .08 & e1.6 & e. 22 & e. 32 & .30 & .12 & .21 \\
\hline 20 & .05 & .06 & .05 & .02 & .09 & .08 & .37 & e. 19 & e. 27 & .13 & .07 & .44 \\
\hline 21 & .05 & .05 & .03 & e. 02 & .07 & .08 & e1. 5 & e. 18 & e. 24 & .06 & .05 & .49 \\
\hline 22 & .05 & .05 & .01 & e. 02 & .07 & .08 & .61 & e. 17 & e. 23 & .05 & .04 & .26 \\
\hline 23 & .06 & .05 & .00 & e. 02 & .07 & .08 & .48 & e. 99 & e. 26 & .05 & .03 & .12 \\
\hline 24 & .06 & .06 & .00 & e. 02 & .06 & .08 & .44 & e. 55 & e. 25 & .05 & .03 & .13 \\
\hline 25 & .06 & .07 & .00 & .03 & .06 & .07 & e. 85 & e. 17 & e. 21 & .05 & .02 & .12 \\
\hline 26 & .06 & .08 & .00 & .03 & .06 & .17 & e2. 3 & e. 56 & e. 22 & .05 & .02 & .11 \\
\hline 27 & .06 & .08 & .00 & .03 & .06 & .12 & .78 & e1.0 & e. 21 & .05 & .02 & .11 \\
\hline 28 & .05 & .07 & .00 & .04 & .08 & .24 & e. 80 & e1.3 & e. 87 & .05 & .02 & .10 \\
\hline 29 & .03 & e. 06 & .00 & .11 & $\cdots$ & .17 & e1.1 & e. 56 & e2.0 & .05 & .02 & .24 \\
\hline 30 & .03 & .06 & .00 & e. 06 & $\cdots$ & .17 & e1.2 & e. 54 & e. 28 & .05 & .02 & .45 \\
\hline 31 & .04 & $\cdots$ & .00 & .07 & $\cdots$ & .09 & $\cdots$ & e. 59 & $\cdots$ & .07 & .02 & $\cdots$ \\
\hline TOTAL & 2.53 & 2.98 & 0.93 & 0.64 & 2.26 & 3.30 & 17.09 & 22.44 & 15.59 & 4.59 & 1.45 & 3.69 \\
\hline MEAN & .082 & .099 & .030 & .021 & .081 & .11 & .57 & .72 & .52 & .15 & .047 & .12 \\
\hline $\operatorname{MAX}$ & .61 & .45 & .06 & .11 & .63 & .38 & 2.3 & 11 & 2.0 & .32 & .29 & .49 \\
\hline MIN & .03 & .04 & .00 & .00 & .00 & .06 & .06 & .12 & .21 & .05 & .02 & .02 \\
\hline$A C-F T^{\prime}$ & 5.0 & 5.9 & 1.8 & .. 3 & 4.5 & 6.5 & 34 & 45 & 31 & 9.1 & 2.9 & 7.3 \\
\hline
\end{tabular}

STATISTICS OF MONTHI,Y MEAN DATA FOR WATER YFARS 1994 - 1995. BY WATER YEAR (WY)

$\begin{array}{lllll}\text { MEAN } & .082 & .099 & .030 & .021 \\ \text { MAX } & .082 & .099 & .030 & .021 \\ \text { (WY) } & 1995 & 1995 & 1995 & 1995 \\ \text { MIN } & .082 & .099 & .030 & .021 \\ \text { (WY) } & 1995 & 1995 & 1995 & 1995\end{array}$

SUMMARY STATISTICS

ANNUAL, TOTAL

ANNUAL MEAN

HIGHEST ANNUAL MEAN

LOWEST ANNUAL MEAN

HIGHEST DAILY MEAN

LOWEST DAILY MEAN

ANNUAL SEVEN-DAY MINIMUM

ANNUAL RUNOFF (AC-FT)

10 PERCENT EXCEEDS

50 PERCENT EXCEEDS

90 PERCENT EXCEEDS

$\begin{array}{rrrr}.081 & .11 & .42 & .43 \\ .081 & .11 & .57 & .72 \\ 1995 & 1995 & 1995 & 1995 \\ .081 & .11 & .26 & .14 \\ 1995 & 1995 & 1994 & 1994\end{array}$

FOR 1995 WATER YEAR

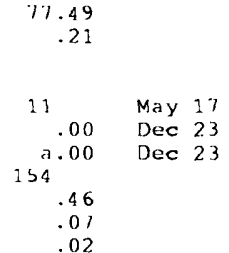

$\begin{array}{rrrr}.30 & .094 & .068 & .094 \\ .52 & .15 & .090 & .12 \\ 1995 & 1995 & 1994 & 1995 \\ .088 & .040 & .047 & .064 \\ 1994 & 1994 & 1995 & 1994\end{array}$

WATER YEARS $1994 \cdot 1995$

a No flow many days

e Estimated 
WALNUT CREEK BELOW POND A-4

SITE NUMBER. - GS11

STATION IDENTIFICATION. - 395403105104700

LOCATION. - Lat $39^{\circ} 54^{\prime} 03^{\prime \prime}$, long $105^{\circ} 10^{\prime} 47^{\prime \prime}$, in $\mathrm{SW}^{1} / 4 \mathrm{SW}^{2} / 4$ sec. $1, \mathrm{~T} .2 \mathrm{~S}$. , R. 70 W., Jefferson County,

Hydrologic Unit 10190003 , Rocky Flats Environmental Technology site, just below Pond A-4 dam.

DRAINAGE AREA. - $0 \mathrm{mi}^{\text {? }}$ (isolated by detention ponds).

PERIOD OF RECORD.--March 1994 to current year.

GAGE. - Water-stage recorder and Parshall flume. Elevation of gage is $5,715 \mathrm{ft}$ above sea level.

REMARKS. - Records fair. Flow regulated by Pond A-4.

DISCHARGE, CUBIC FEET PER SECIND, WATER YEAR (OCTOBER 1993 TO SEPTEMBER 1994

\begin{tabular}{|c|c|c|c|c|c|c|c|c|c|c|c|c|}
\hline DAY & $(x \mathrm{~T}$ & Nov & DEC & JAN & $9: 3$ & MAR & $A P R$ & MAY & JUN & JUL & AUG & SEP \\
\hline 1 & $\cdots$ & $\cdots$ & $\cdots$ & $\cdots$ & $\cdots$ & $\cdots$ & 3.9 & .00 & .00 & .00 & .02 & .00 \\
\hline 2 & $\cdots$ & $\cdots$ & $\cdots$ & $\cdots$ & $\cdots$ & $\cdots$ & 1.8 & .00 & .00 & .00 & .01 & .00 \\
\hline 3 & $\cdots$ & $\cdots$ & $\cdots$ & $\cdots$ & $\cdots$ & $\cdots$ & 1.8 & .00 & .00 & .00 & .01 & .00 \\
\hline 4 & $\cdots$ & $\cdots$ & $\cdots$ & $\cdots$ & $\cdots$ & $\cdots$ & 1.8 & .00 & .00 & .00 & .00 & .00 \\
\hline 5 & $\cdots$ & $\cdots$ & $\cdots$ & $\cdots$ & $\cdots$ & $\cdots$ & 1.8 & .00 & .00 & .00 & .00 & .00 \\
\hline 6 & $\cdots$ & $\cdots$ & $\cdots$ & $\cdots$ & $\cdots$ & $\cdots$ & 1.8 & .00 & .00 & .00 & .00 & .00 \\
\hline 7 & $\cdots$ & $\cdots$ & $\cdots$ & $\cdots$ & $\cdots$ & $\cdots$ & 1.7 & .00 & .00 & .00 & .00 & 1.2 \\
\hline 8 & $\cdots$ & $\cdots$ & $\cdots$ & $\ldots$ & $\cdots$ & $\cdots$ & 1.6 & .00 & .00 & .00 & .00 & 1.7 \\
\hline 9 & $\cdots$ & $\cdots$ & $\cdots$ & $\cdots$ & $\cdots$ & $\cdots$ & 1.7 & .00 & .00 & .00 & .00 & 1.6 \\
\hline 10 & $\cdots$ & $\cdots$ & $\cdots$ & $\cdots$ & $\cdots$ & $\cdots$ & 1.6 & .00 & .00 & .00 & .00 & 1.4 \\
\hline 11 & $\cdots$ & $\cdots$ & $\cdots$ & $\cdots$ & $\cdots$ & $\cdots$ & 1.6 & .00 & .00 & .00 & .03 & 1.4 \\
\hline 12 & $\cdots$ & $\cdots$ & $\cdots$ & $\cdots$ & $\cdots$ & $\cdots$ & 1.5 & .00 & .00 & .00 & .01 & 1.4 \\
\hline 13 & $\cdots$ & $\cdots$ & $\cdots$ & $\cdots$ & $\cdots$ & $\cdots$ & .47 & .00 & .00 & .00 & .00 & 1.4 \\
\hline 14 & $\cdots$ & $\cdots$ & $\cdots$ & $\cdots$ & $\cdots$ & $\cdots$ & .00 & 1.5 & .00 & .00 & .00 & 3.5 \\
\hline 15 & $\cdots$ & $\cdots$ & $\cdots$ & $\cdots$ & $\cdots$ & $\cdots$ & .00 & 2.1 & .00 & .00 & .00 & 1.5 \\
\hline 16 & $\cdots$ & $\cdots$ & $\cdots$ & $\cdots$ & $\cdots$ & .00 & .00 & 2.0 & .00 & .00 & .00 & .85 \\
\hline 17 & $\cdots$ & $\cdots$ & $\cdots$ & $\cdots$ & $\cdots$ & .00 & .00 & 2.0 & .00 & .00 & .00 & .01 \\
\hline 18 & $\cdots$ & $\cdots$ & $\cdots$ & $\cdots$ & $\cdots$ & .00 & .00 & 1.9 & 1.4 & .00 & .00 & .00 \\
\hline 19 & $\cdots$ & $\cdots$ & $\cdots$ & $\cdots$ & $\cdots$ & .00 & .00 & 1.9 & 2.0 & .00 & .00 & .00 \\
\hline 20 & $\cdots$ & $\cdots$ & $\cdots$ & $\cdots$ & $\cdots$ & .00 & .00 & .92 & 1.9 & .00 & .00 & .00 \\
\hline 21 & $\cdots$ & $\cdots$ & $\cdots$ & $\cdots$ & $\cdots$ & .00 & .00 & .00 & 1.9 & .00 & .00 & .00 \\
\hline 22 & $\cdots$ & $\cdots$ & $\cdots$ & $\ldots$ & $\cdots$ & .00 & .00 & .00 & 1.9 & .00 & .00 & .00 \\
\hline 2.3 & $\cdots$ & $\cdots$ & $\cdots$ & $\cdots$ & $\cdots$ & 1.2 & .00 & .00 & 1.9 & 1.3 & .00 & .00 \\
\hline 24 & $\cdots$ & $\cdots$ & $\cdots$ & $\cdots$ & $\cdots$ & 1.9 & .00 & .00 & 1.9 & 1.7 & .00 & .00 \\
\hline 25 & $\cdots$ & $\cdots$ & $\cdots$ & $\cdots$ & $\cdots$ & 1.9 & .01 & .00 & 1.9 & 1.7 & .00 & .00 \\
\hline 26 & $\cdots$ & $\cdots$ & $\cdots$ & $\cdots$ & $\cdots$ & 1.9 & .00 & .00 & 2.0 & 1.6 & .00 & .00 \\
\hline 27 & $\cdots$ & $\cdots$ & $\cdots$ & $\cdots$ & $\cdots$ & 1.9 & .00 & .00 & .98 & 1.7 & .00 & .00 \\
\hline 28 & $\cdots$ & $\cdots$ & $\cdots$ & $\cdots$ & $\cdots$ & 1.9 & .00 & .00 & .00 & 1.7 & .00 & .00 \\
\hline 29 & $\cdots$ & $\cdots$ & $\cdots$ & $\cdots$ & $\cdots$ & 1.9 & .00 & .00 & .00 & 1.6 & .00 & .00 \\
\hline 30 & $\cdots$ & $\cdots$ & $\cdots$ & $\cdots$ & $\cdots$ & 1.9 & .00 & .00 & .00 & 1.6 & .00 & .00 \\
\hline 31 & $\cdots$ & $\cdots$ & $\cdots$ & $\cdots$ & $\cdots$ & 1.9 & $\cdots$ & .00 & $\cdots$ & 1.0 & .00 & $\cdots$ \\
\hline TOTAL & $\cdots$ & $\cdots$ & $\cdots$ & $\cdots$ & $\cdots$ & $\cdots$ & 21.08 & 12.32 & 17.78 & 13.90 & 0.08 & 13.96 \\
\hline MEAN & $\cdots$ & $\cdots$ & $\cdots$ & $\cdots$ & $\cdots$ & $\cdots$ & .70 & .40 & .59 & .45 & .003 & .47 \\
\hline MAX & $\cdots$ & $\cdots$ & $\cdots$ & $\cdots$ & $\cdots$ & $\cdots$ & 1.9 & 2.1 & 2.0 & 1.7 & .03 & 1.7 \\
\hline MIN & $\cdots$ & $\cdots$ & $\cdots$ & $\cdots$ & $\cdots$ & $\cdots$ & .00 & . 00 & .00 & .00 & .00 & .00 \\
\hline $\mathrm{AC}-\mathrm{FT}$ & $\cdots$ & $\cdots$ & $\cdots$ & $\cdots$ & $\cdots$ & $\cdots$ & 42 & 24 & 35 & 28 & .2 & 28 \\
\hline
\end{tabular}


SITE NUMBER. - GS11

STATION IDENTIFICATION. - - 395403105104700

LOCATION. - Lat $39^{\circ} 54^{\prime} 03^{\prime \prime}$, Long $105^{\circ} 10^{\prime} 47^{\prime \prime}$, in $\mathrm{SW}^{1} / 4 \mathrm{SW}^{1} / 4 \mathrm{sec} .1, \mathrm{~T} .2 \mathrm{~S} ., \mathrm{R} .70 \mathrm{~W} ., \mathrm{Jefferson}$ County. Hydrologic Unit 10190003, Rocky Flats Environmental Technology Site, just below Pond A-4 dam.

DRAINAGE AREA. - $0 \mathrm{mi}^{2}$ (isolated by detention ponds) .

PERIOD OF RECORD. - March 1994 to current year.

GAGE. - Water-stage recorder and Parshall flume. Elevation of gage is $5,715 \mathrm{ft}$ above sea level.

REMARKS. - Records fair except for estimated daily discharges, which are poor. DISCHARGE, CUBIC FEET PER SECOND, WATER YEAR OCTUBER 1994 TO SEPTEMBER 1995

\begin{tabular}{|c|c|c|c|c|c|c|c|c|c|c|c|c|}
\hline DAY & WCT & NOV & DEC & JAN & FEB & MAR & $A P R$ & MAY & JUN & JUL & AUG & SEP \\
\hline 1 & e. 00 & e. 00 & .00 & .00 & .00 & e. 00 & .00 & 3.7 & 3.1 & .00 & e. 00 & .00 \\
\hline 2 & e. 00 & e. 00 & .00 & .00 & .00 & .00 & .00 & 3.6 & 3.3 & .00 & e1. 5 & .00 \\
\hline 3 & .00 & e. 00 & .00 & e. 00 & .00 & e. 00 & .00 & 3.5 & 2.5 & .00 & $\mathrm{e} 2.7$ & .00 \\
\hline 4 & .00 & e. 00 & .00 & c. 00 & .00 & e. 00 & .00 & 3.4 & 2.9 & .00 & e2. 4 & .00 \\
\hline 5 & .00 & e. 00 & .00 & e. 00 & .00 & e. 00 & .00 & 3.2 & 2.8 & .00 & e2. 3 & .00 \\
\hline 6 & .00 & e. 00 & .00 & e. 00 & .00 & e. 00 & .00 & 2.0 & 2.8 & .00 & e2.1 & .00 \\
\hline 7 & .00 & e. 00 & .00 & e. 00 & .00 & .00 & .00 & 1.9 & 2.8 & e. 00 & e2.1 & .00 \\
\hline 8 & .00 & e. 00 & .00 & .00 & 1.4 & .00 & .00 & 2.1 & 2.8 & e. 00 & 2.1 & .00 \\
\hline 9 & .00 & e. 00 & .00 & .00 & 2.0 & .00 & .00 & .86 & 2.8 & e. 00 & 2.1 & .00 \\
\hline 10 & .00 & e. 00 & .89 & .00 & 2.0 & 1.4 & .00 & e. 00 & 2.8 & e. 00 & 1.8 & .01 \\
\hline 11 & e. 00 & e. 00 & 1.9 & .00 & 1.9 & 1.9 & .00 & e. 00 & 2.7 & e. 00 & 1.4 & .01 \\
\hline 12 & e. 00 & .00 & 1.9 & .00 & 1.7 & 1.1 & .00 & e. 00 & 2.5 & e. 00 & .87 & .00 \\
\hline 13 & e. 00 & .00 & 1.8 & .00 & 1.3 & 1.6 & .00 & e. 00 & 2.6 & e. 00 & .58 & .00 \\
\hline 14 & e. 00 & .00 & 1.6 & .00 & .05 & 1.5 & 1.6 & e. 00 & 2.6 & .00 & e. 00 & .00 \\
\hline 15 & e. 00 & .00 & 1.5 & .00 & .05 & 1.3 & 2.3 & e. 00 & 2.6 & .00 & .00 & 2.9 \\
\hline 16 & e. 00 & .00 & 1.9 & .00 & .04 & 1.2 & 2.1 & e. 00 & e1. 3 & .00 & e. 00 & 3.8 \\
\hline 17 & e. 00 & .00 & 2.0 & .00 & .04 & .63 & 2.0 & 1.2 & e. 00 & .00 & e. 00 & 3.3 \\
\hline 18 & e. 00 & .00 & 2.0 & .00 & .03 & .04 & 2.0 & 3.9 & e. 00 & .00 & e. 00 & 2.7 \\
\hline 19 & e. 00 & .00 & 1.4 & .00 & .03 & .03 & 1.9 & 3.9 & e. 00 & .00 & .00 & 2.6 \\
\hline 20 & e. 00 & .00 & 1.4 & .00 & .03 & .02 & 1.4 & 3.9 & .00 & .00 & .00 & 2.3 \\
\hline 2.1 & .00 & .00 & 1.3 & .00 & e. 00 & e. 00 & .69 & 3.8 & .00 & .00 & e. 00 & 2.3 \\
\hline 22 & .90 & .00 & .54 & .00 & e. 00 & .00 & .18 & 3.9 & .00 & .00 & e. 00 & 2.0 \\
\hline 23 & 1.4 & .00 & .02 & .00 & e. 00 & .00 & .16 & 3.9 & .00 & .00 & e. 00 & 1.7 \\
\hline 24 & 1.4 & .00 & .01 & .00 & e. 00 & .00 & .16 & 3.9 & e. 00 & .00 & e. 00 & 1.7 \\
\hline 25 & 1.8 & .00 & .01 & .00 & e. 00 & .00 & .16 & 3.9 & e. 00 & .00 & e. 00 & 1.5 \\
\hline 26 & 2.0 & .00 & .01 & .00 & e. 00 & .00 & 1.0 & 3.7 & e. 00 & .00 & e. 00 & 1.3 \\
\hline 27 & 2.0 & .00 & .01 & .00 & .00 & .00 & 2.6 & 3.4 & e. 00 & .00 & e. 00 & .74 \\
\hline 28 & 1.8 & .00 & .01 & .00 & e. 00 & .00 & 3.3 & 3.2 & .00 & .00 & e. 00 & .00 \\
\hline 29 & 1.7 & .00 & .01 & .00 & . & .00 & 3.5 & 3.0 & .00 & .00 & e. 00 & .00 \\
\hline 30 & 1.7 & .00 & .01 & .00 & $\ldots$ & .00 & 3.6 & 2.9 & .00 & .00 & e. 00 & .00 \\
\hline 31 & .94 & $\cdots$ & .00 & .00 & $\cdots$ & .00 & $\cdots$ & 2.9 & $\cdots$ & .00 & .00 & $\cdots$ \\
\hline TOTAL & 15.64 & 0.00 & 20.22 & 0.00 & 10.57 & 11.32 & 28.65 & 75.66 & 42.90 & 0.00 & 21.95 & 28.86 \\
\hline MEAN & .50 & .000 & .65 & .000 & .38 & .37 & .95 & 2.44 & 1.43 & .000 & .71 & .96 \\
\hline $\operatorname{MAX}$ & 2.0 & .00 & 2.0 & .00 & 2.0 & 1.9 & 3.6 & 3.9 & 3.3 & .00 & 2.7 & 3.8 \\
\hline MIN & .00 & .00 & .00 & .00 & .00 & .00 & .00 & .00 & .00 & .00 & .00 & .00 \\
\hline$A C-F T$ & 31 & .00 & 40 & .00 & 21 & 22 & 57 & 150 & 85 & .00 & 44 & 57 \\
\hline
\end{tabular}

STATISTICS OF MONTHLY MEAN DATA FOR WATER YEARS 1994 - 1995, BY WATER YEAR (WY)

$\begin{array}{lrrrr}\text { MFAN } & .50 & .000 & .65 & .000 \\ \text { MAX } & .50 & .000 & .65 & .000 \\ \text { (WY) } & 1995 & 1995 & 1995 & 1995 \\ \text { MIN } & .50 & .000 & .65 & .000 \\ \text { (WY) } & 1995 & 1995 & 1995 & 1995\end{array}$

SUMMARY STATISTICS

ANNUAL TOT'AL

ANNUAL MEAN

HIGHEST ANNUAL MEAN

LOWEST ANNUAL MEAN

HIGHEST DAILY MEAN

LOWEST DAILY MEAN

ANNUAL SEVEN-DAY MINIMUM

ANNUAL RUNOFF (AC・FT)

10 PERCENT EXCEEDS

50 PERCENT EXCEEDS

90 PERCENT EXCEEDS

$\begin{array}{rr}.38 & .37 \\ .38 & .37 \\ 1995 & 1995 \\ .38 & .37 \\ 1995 & 1995\end{array}$

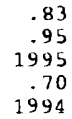

1.42
2.44
1995
.40
1994

FOR 1995 WATER YEAR

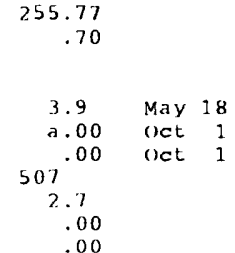

1.01
1.43
1995
.59
1994
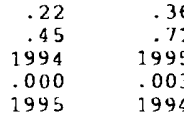

36
.71
995

.003
1994

1995

.47
1994

a No flow many days

e Estimateri 
WALNUT CREEK BELOW POND A-3

SITE NUMBER. - - GS12

STATION IDENTIFICATION. - - 395358105110500

LOCATION. - Lat $39^{\circ} 53^{\prime} 58^{\prime \prime}$, long $105^{\circ} 11^{\prime} 05^{\prime \prime}$, in $\mathrm{NE}^{1} / 4 \mathrm{NE}^{1} / 4$ sec.11, T. 2 S., R. 70 W., Jefferson County, Hydrologic unit 10190003, Rocky Flats Environmental Technology site, just downstream from Pond A- 3 dam.

DRAINAGE AREA. - - $0 \mathrm{mi}^{2}$ (isolated by detention ponds) .

PERIOD OF RECORD. - February 1994 to current year.

GAGE. - Water-stage recorder and Parshall flume. Elevation of gage is $5,761 \mathrm{ft}$ above sea level.

REMARKS. - Records poor.

DISCHARGE, CUBIC PEET PER SECOND, WA'TER YEAR OCTOBER 1993 TO SEPTEMBER 1994

\begin{tabular}{|c|c|c|c|c|c|c|c|c|c|c|c|c|}
\hline DAY & $O C T$ & $\mathrm{NON}$ & DEC & JAN & FEB & MAR & $\mathrm{A} P \mathrm{PR}$ & MAY & JUN & JUL, & $A \cup G$ & SEP \\
\hline 1 & $\cdots$ & $\cdots$ & $\cdots$ & $\cdots$ & $\cdots$ & e. 00 & e. 00 & .00 & .02 & .94 & .00 & e. 00 \\
\hline 2 & $\cdots$ & $\cdots$ & $\cdots$ & $\cdots$ & $\ldots$ & e. 00 & e. 00 & .00 & .01 & .00 & .00 & e. 00 \\
\hline 3 & $\ldots$ & $\cdots$ & $\cdots$ & $\ldots$ & $\cdots$ & .12 & e. 00 & .00 & .12 & .00 & .00 & e. 00 \\
\hline 4 & $\cdots$ & $\cdots$ & $\cdots$ & $\ldots$ & $\ldots$ & .07 & e. 00 & .00 & e. 00 & .00 & .00 & e. 00 \\
\hline 5 & $\cdots$ & $\cdots$ & $\cdots$ & $\cdots$ & $\cdots$ & e. 00 & .03 & .00 & .00 & .00 & .00 & e. 00 \\
\hline 6 & $\cdots$ & $\ldots$ & $\cdots$ & $\cdots$ & $\cdots$ & e. 00 & .04 & .00 & e. 00 & .00 & .00 & e. 00 \\
\hline 7 & $\cdots$ & $\cdots$ & $\cdots$ & $\cdots$ & $\cdots$ & e. 00 & e. 00 & .00 & .00 & e. 00 & .00 & e. 00 \\
\hline 8 & $\cdots$ & $\cdots$ & $\cdots$ & $\cdots$ & $\cdots$ & .07 & .04 & .00 & e. 00 & e. 00 & 1.2 & e. 00 \\
\hline 9 & $\cdots$ & $\cdots$ & $\cdots$ & $\cdots$ & $\cdots$ & 1.1 & e. 00 & .00 & e. 00 & e. 00 & 1.8 & .00 \\
\hline 10 & $\cdots$ & $\cdots$ & $\cdots$ & $\cdots$ & $\cdots$ & .44 & e. 00 & .00 & e. 00 & .00 & .88 & .00 \\
\hline 11 & $\cdots$ & $\cdots$ & $\cdots$ & $\cdots$ & $\cdots$ & e. 00 & e. 00 & .00 & e. 00 & e. 00 & .09 & .00 \\
\hline 12 & $\cdots$ & $\cdots$ & $\cdots$ & $\cdots$ & $\cdots$ & e. 00 & .04 & .00 & .00 & .00 & .04 & .00 \\
\hline 13 & $\cdots$ & $\cdots$ & $\cdots$ & $\cdots$ & $\cdots$ & .12 & 1.3 & .00 & .00 & e. 00 & e. 00 & .00 \\
\hline 14 & $\cdots$ & $\cdots$ & $\cdots$ & $\cdots$ & $\cdots$ & e. 00 & 2.1 & .00 & e. 00 & .00 & .00 & .00 \\
\hline 15 & $\cdots$ & $\cdots$ & $\cdots$ & $\cdots$ & $\cdots$ & .03 & 1.9 & .00 & e. 00 & .00 & .00 & .00 \\
\hline 16 & $\cdots$ & $\cdots$ & $\cdots$ & $\cdots$ & $\cdots$ & e. 00 & 1.7 & .00 & .00 & .00 & .00 & .00 \\
\hline 17 & $\cdots$ & $\cdots$ & $\ldots$ & $\cdots$ & $\cdots$ & e. 00 & 1.6 & .00 & .00 & .00 & .00 & .00 \\
\hline 18 & $\cdots$ & $\cdots$ & $\cdots$ & $\cdots$ & $\cdots$ & e. 00 & 1.3 & .03 & .00 & .00 & .00 & .00 \\
\hline 19 & $\cdots$ & $\cdots$ & $\cdots$ & $\cdots$ & $\cdots$ & .00 & 1.2 & .07 & .00 & e. 00 & .00 & .00 \\
\hline 20 & $\cdots$ & $\cdots$ & $\cdots$ & $\cdots$ & $\cdots$ & e. 00 & e1.0 & .04 & .00 & .00 & .00 & .00 \\
\hline 21 & $\cdots$ & $\cdots$ & $\cdots$ & $\cdots$ & $\cdots$ & e. 00 & e. 00 & .00 & .01 & .00 & .00 & .00 \\
\hline 22 & $\cdots$ & $\cdots$ & $\cdots$ & $\cdots$ & $\cdots$ & e. 00 & e. 00 & .00 & .00 & .00 & .00 & .00 \\
\hline 23 & $\cdots$ & $\cdots$ & $\cdots$ & $\cdots$ & $\cdots$ & e. 00 & e. 00 & 1.0 & .00 & .00 & .00 & .00 \\
\hline 24 & $\cdots$ & $\cdots$ & $\cdots$ & $\cdots$ & $\cdots$ & e. 00 & e. 00 & 1.5 & .00 & .00 & .00 & .00 \\
\hline 25 & $\cdots$ & $\cdots$ & $\cdots$ & $\cdots$ & $\cdots$ & e. 00 & e. 00 & 1.4 & .00 & .00 & .00 & .00 \\
\hline 26 & $\cdots$ & $\cdots$ & $\cdots$ & $\cdots$ & $\cdots$ & e. 00 & e. 00 & 1.4 & .00 & .00 & .00 & .00 \\
\hline 27 & $\cdots$ & $\cdots$ & $\cdots$ & $\cdots$ & $\cdots$ & e. 00 & e. 00 & .76 & .00 & .00 & .00 & .00 \\
\hline 28 & $\cdots$ & $\cdots$ & $\cdots$ & $\cdots$ & $\cdots$ & e. 00 & .00 & .08 & 1.2 & .00 & .00 & .00 \\
\hline 29 & $\cdots$ & $\cdots$ & $\cdots$ & $\cdots$ & $\cdots$ & e. 00 & e. 00 & .02 & 1.7 & .00 & .00 & .69 \\
\hline 30 & $\cdots$ & $\cdots$ & $\cdots$ & $\cdots$ & $\cdots$ & e. 00 & .00 & .00 & 1.6 & .00 & e. 00 & 1.4 \\
\hline 31 & $\cdots$ & $\cdots$ & $\cdots$ & $\cdots$ & $\cdots$ & .02 & $\cdots$ & .02 & $\cdots$ & .00 & e. 00 & $\cdots$ \\
\hline TYTAL & $\cdots$ & $\cdots$ & $\cdots$ & $\cdots$ & $\cdots$ & 1.97 & 12.25 & 6.32 & 4.66 & 0.94 & 4.01 & 2.09 \\
\hline MEAN & $\cdots$ & $\cdots$ & $\cdots$ & $\cdots$ & $\cdots$ & .064 & .41 & .20 & .16 & .030 & .13 & .070 \\
\hline MAX & $\cdots$ & $\cdots$ & $\cdots$ & $\cdots$ & $\cdots$ & 1.1 & 2.1 & 1.5 & 1.7 & .94 & 1.8 & 1.4 \\
\hline MIN & $\cdots$ & $\cdots$ & $\cdots$ & $\cdots$ & $\cdots$ & .00 & .00 & .00 & .00 & .00 & .00 & .00 \\
\hline$A C-F^{\prime} T$ & $\cdots$ & $\cdots$ & $\cdots$ & $\cdots$ & $\cdots$ & 3.9 & 24 & 13 & 9.2 & 1.9 & 8.0 & 4.1 \\
\hline
\end{tabular}

e Estimater] 
WALNUT CREEK BELOW POND A-3

SITE NUMBER. - - GS12

STATION IDENTIFICATION. - - 395358105110500

LOCATION. - Lat $39^{\circ} 53^{\prime} 58^{\prime \prime}$, long $105^{\circ} 11^{\prime} 05^{\prime \prime}$, in $\mathrm{NE}^{1} / 4 \mathrm{NE}^{1} / 4$ sec.11, T.2 S., R. 70 w. Jefferson County, Hydrologic Unit 10190003, Rocky Flats Environmental Technology Site, just downstream from Pond A -3 dam.

DRAINAGE AREA. - $0 \mathrm{mi}^{2}$ (isolated by detention ponds).

PERIOD OF RECORD. - February 1994 to current year.

GAGE. - Water-stage recorder and Parshall flume. Elevation of gage is 5,761 ft above sea level.

REMARKS. - Records poor.

DISCHARGE, CUBIC FEET PER SFCOND, WATER YEAR OCTOBER :994 TO SEPTEMBFR 1995

\begin{tabular}{|c|c|c|c|c|c|c|c|c|c|c|c|c|}
\hline DAY & $O C T$ & $\mathrm{NOV}$ & $\mathrm{DFC}$ & JAN & PEB & MAR & $A P R$ & MAY & IUN & JUI, & $A \cup G$ & SEP \\
\hline 1 & 1.4 & .00 & .00 & .00 & .00 & .00 & .00 & 2.3 & e2.6 & .17 & .00 & .05 \\
\hline 2 & 1.4 & .00 & .00 & .00 & .00 & .00 & .00 & 2.3 & e2. 6 & .17 & .00 & .06 \\
\hline 3 & .82 & .00 & .00 & .00 & .00 & .00 & .00 & 2.3 & e2. 6 & .19 & .00 & .07 \\
\hline 4 & .00 & .00 & .00 & .00 & .00 & .00 & .00 & 2.3 & e2. 6 & .18 & .00 & .05 \\
\hline b & .00 & .00 & .00 & .00 & .00 & .00 & .00 & e1.0 & e2. o & .15 & .00 & .05 \\
\hline 6 & .00 & .00 & .00 & .00 & .00 & .00 & .00 & .00 & 2.6 & .14 & .00 & .07 \\
\hline 7 & .00 & .00 & .00 & .00 & .00 & .00 & .00 & .00 & 2.5 & .14 & .00 & .11 \\
\hline 8 & .00 & .00 & .00 & .00 & .00 & .00 & .00 & .00 & 1.5 & .13 & .00 & .07 \\
\hline 9 & .00 & .00 & .00 & .00 & .00 & .00 & .00 & .00 & 2.9 & .14 & .00 & .08 \\
\hline 10 & .00 & .00 & .00 & .00 & .00 & .00 & .00 & .00 & 2.3 & .14 & .00 & .08 \\
\hline 11 & .00 & .00 & .00 & .00 & .00 & .00 & .00 & .00 & 1.2 & 1.5 & .00 & .08 \\
\hline 12 & .00 & .00 & .00 & .00 & .00 & .00 & .00 & .00 & 1.2 & 2.0 & .00 & .06 \\
\hline 13 & .00 & .00 & .00 & .47 & .00 & .00 & .00 & .00 & 1.0 & 1.8 & .00 & .07 \\
\hline 14 & .00 & .00 & $.0 n$ & .90 & .00 & .00 & .00 & .00 & .92 & .91 & 1.2 & .07 \\
\hline 15 & .00 & .00 & .00 & .91 & .00 & .00 & .00 & .00 & .75 & .16 & 1.6 & .06 \\
\hline 16 & .00 & .00 & .00 & .89 & .00 & .00 & .00 & .00 & .14 & .17 & 1.6 & .06 \\
\hline 17 & .00 & .00 & .00 & .54 & .00 & .00 & .00 & e8.0 & .18 & .16 & .00 & .06 \\
\hline 18 & .00 & .00 & .00 & .00 & .00 & .00 & .00 & 18 & .16 & .18 & .00 & .09 \\
\hline 19 & .00 & .00 & .00 & .00 & .00 & .00 & .00 & 18 & .13 & .20 & .00 & .12 \\
\hline 20 & .00 & .00 & .00 & .00 & .00 & .00 & .00 & 18 & .13 & .18 & .00 & .15 \\
\hline 21 & .00 & .00 & .00 & .00 & .00 & .00 & .00 & 18 & .14 & .10 & .00 & .20 \\
\hline 22 & .00 & .00 & .00 & .00 & .00 & .00 & .00 & e8.0 & .16 & .08 & .00 & .17 \\
\hline 23 & .00 & .00 & .00 & .00 & .00 & 1.1 & .00 & e2.6 & .16 & .16 & .00 & .13 \\
\hline 24 & .00 & .00 & .00 & .00 & .00 & 1.8 & .00 & e2 .6 & .19 & .17 & .00 & .13 \\
\hline 25 & .00 & .00 & .00 & .00 & .00 & 1.8 & .00 & e2. 6 & .16 & .14 & .00 & .12 \\
\hline 26 & .00 & .00 & .00 & .00 & .00 & 1.8 & e. 90 & $\mathrm{e} 2.6$ & .15 & .14 & .00 & .11 \\
\hline 27 & .00 & .00 & .00 & .00 & .00 & 1.8 & e 2.3 & $\mathrm{e} 2.6$ & .16 & .14 & .00 & .09 \\
\hline 28 & .00 & .00 & .00 & .00 & .00 & 1.8 & 2.3 & e 2.6 & .22 & .15 & .00 & .10 \\
\hline 29 & .00 & .00 & .00 & .00 & $\ldots$ & 1.8 & 2.3 & $\mathrm{e} 2.6$ & .25 & .13 & .00 & .11 \\
\hline 30 & .00 & .00 & .00 & .00 & $\ldots$ & 1.8 & 2.3 & e 2.6 & .23 & .15 & .00 & .12 \\
\hline 31 & .00 & . & .00 & .00 & $\cdots$ & .85 & 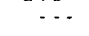 & e 2.6 & $\cdots$ & .24 & .00 & $\cdots$ \\
\hline TOTAL & 3.62 & 0.00 & 0.00 & 3.71 & 0.00 & 14.55 & 10.10 & 121.60 & 32.43 & 10.41 & 4.40 & 2.79 \\
\hline MEAN & .12 & .000 & .000 & .12 & .000 & .47 & .34 & 3.92 & 1.08 & .34 & .14 & .093 \\
\hline MAX & 1.4 & .00 & .00 & .91 & .00 & 1.8 & 2.3 & 18 & 2.9 & 2.0 & 1.6 & .20 \\
\hline MIN & .00 & .00 & .00 & .00 & .00 & .00 & .00 & .00 & .13 & .08 & .00 & .05 \\
\hline$A C \cdot F T$ & 7.2 & .00 & .00 & 7.4 & .00 & 29 & 20 & 241 & 64 & 21 & 8.7 & 5.5 \\
\hline
\end{tabular}

STATISTICS (OF MUNTHI,Y MEAN DATA FOR WATER YEARS 1994 - 1995, BY WATER YEAR (WY)

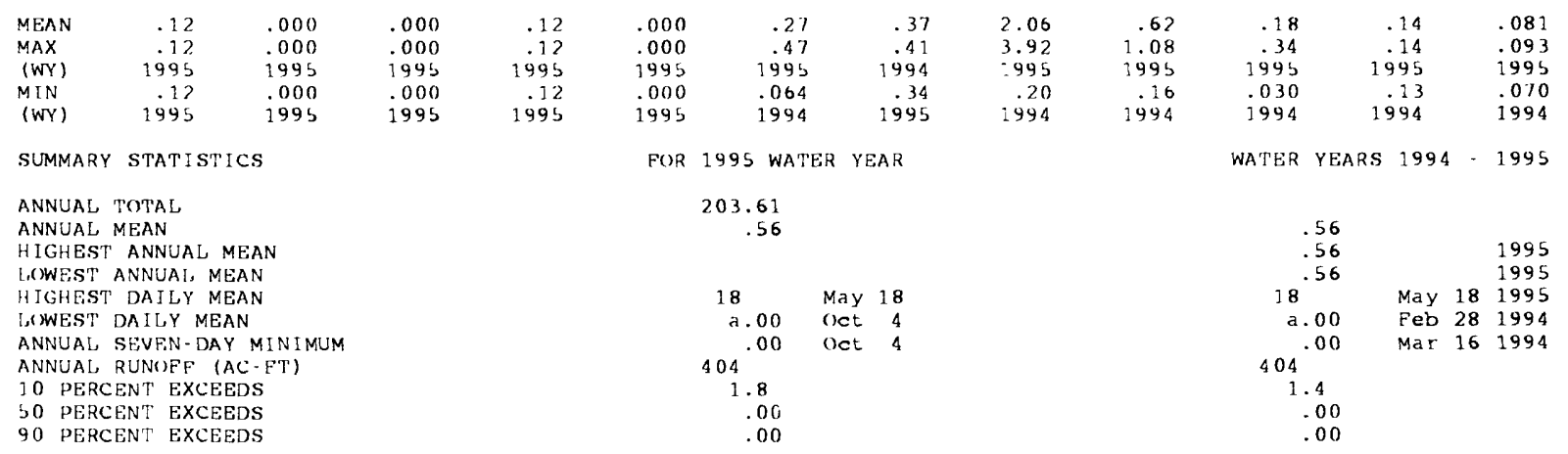

a No flow many days 
SITE NUMBER. - - GS14

STATION IDENTIFICATION. - 395304105105100

LOCATION. - Lat $39^{\circ} 53^{\prime} 04^{\prime \prime}$, long $105^{\circ} 10^{\prime} 51^{\prime \prime}$, in $\mathrm{NW}^{2} / 4 \mathrm{NW}^{1} / 4$ sec. $13, \mathrm{~T} .2$ S., R. 70 W., Jefferson County, Hydrologic Unit 10190003, Rocky Flats Environmental Technology site, between Woman Creek Diversion and Mower Diversion.

DRAINAGE AREA. - - $1.4 \mathrm{mi}^{2}$.

PERIOD OF RECORD.--March 1994 to current year.

GAGE. - Water-stage recorder and Parshall flume with weir plate. Elevation of gage is 5,705 ft above sea level.

REMARKS. - Records poor.

DISCHARGE, CUBIC FEET PER SEC(NND, WATER YEAR (OCTOBER I 993 TO SEPTEMBER 1994

\begin{tabular}{|c|c|c|c|c|c|c|c|c|c|c|c|c|}
\hline DAY & (XCT & NoV & DEC & JAN & FEB & MAR & APR & MAY & JUN & JUL & $A \cup G$ & SEP \\
\hline 1 & $\ldots$ & $\cdots$ & $\cdots$ & $\cdots$ & $\ldots$ & $\ldots$ & .53 & 1.6 & .04 & .00 & .00 & .00 \\
\hline 2 & $\ldots$ & $\ldots$ & $\ldots$ & $\ldots$ & $\ldots$ & $\ldots$ & .39 & 1.2 & .02 & .00 & .00 & .00 \\
\hline 3 & $\cdots$ & $\cdots$ & $\cdots$ & $\cdots$ & $\cdots$ & $\cdots$ & .36 & 1.3 & .03 & .00 & .00 & .00 \\
\hline 4 & $\cdots$ & $\cdots$ & $\ldots$ & $\ldots$ & $\ldots$ & $\ldots$ & .28 & .89 & .07 & .00 & .00 & .00 \\
\hline 5 & $\ldots$ & $\cdots$ & $\cdots$ & $\cdots$ & $\cdots$ & $\cdots$ & .30 & .75 & .03 & .00 & .00 & .00 \\
\hline 6 & $\cdots$ & $\ldots$ & $\ldots$ & $\ldots$ & $\ldots$ & $\cdots$ & .48 & .72 & .00 & .00 & .00 & .00 \\
\hline 7 & $\cdots$ & $\ldots$ & $\ldots$ & $\ldots$ & $\cdots$ & $\ldots$ & .52 & .57 & .00 & .00 & .00 & .00 \\
\hline 8 & $\ldots$ & $\cdots$ & $\cdots$ & $\cdots$ & $\cdots$ & $\cdots$ & .31 & .48 & .00 & .00 & .00 & .00 \\
\hline 9 & $\ldots$ & $\cdots$ & $\cdots$ & $\ldots$ & $\cdots$ & $\cdots$ & .30 & .47 & .00 & .00 & .00 & .00 \\
\hline 10 & $\cdots$ & $\cdots$ & $\cdots$ & $\cdots$ & $\cdots$ & $\cdots$ & .71 & .56 & .00 & .00 & .00 & .00 \\
\hline 11 & $\cdots$ & $\cdots$ & $\cdots$ & $\cdots$ & $\cdots$ & $\cdots$ & .82 & .41 & .00 & .00 & .00 & .00 \\
\hline 12 & $\cdots$ & $\ldots$ & $\ldots$ & $\ldots$ & $\ldots$ & $\ldots$ & 1.7 & .36 & .00 & .00 & .00 & .00 \\
\hline 13 & $\cdots$ & $\cdots$ & $\cdots$ & $\ldots$ & $\ldots$ & $\ldots$ & 2.1 & .35 & .00 & .00 & .00 & .00 \\
\hline 14 & $\cdots$ & $\ldots$ & $\cdots$ & $\ldots$ & $\ldots$ & $\ldots$ & .93 & .68 & .00 & .00 & .00 & .00 \\
\hline 15 & $\cdots$ & $\cdots$ & $\cdots$ & $\cdots$ & $\cdots$ & $\cdots$ & .66 & .34 & .00 & .00 & .00 & .00 \\
\hline 16 & $\cdots$ & $\cdots$ & $\cdots$ & $\cdots$ & $\ldots$ & .24 & .51 & .24 & .00 & .00 & .00 & .00 \\
\hline 17 & $\cdots$ & $\cdots$ & $\cdots$ & $\cdots$ & $\cdots$ & .22 & .42 & .16 & .00 & .00 & .00 & .00 \\
\hline 18 & $\cdots$ & $\cdots$ & $\ldots$ & $\cdots$ & $\ldots$ & .18 & .41 & .10 & .00 & .00 & .00 & .00 \\
\hline 19 & $\ldots$ & . . & $\cdots$ & $\cdots$ & $\ldots$ & .17 & .36 & .06 & .00 & .00 & .00 & .00 \\
\hline 20 & $\cdots$ & $\cdots$ & $\cdots$ & $\cdots$ & $\cdots$ & .15 & .38 & .04 & .00 & .00 & .00 & .00 \\
\hline 21 & $\ldots$ & $\cdots$ & $\cdots$ & $\cdots$ & $\cdots$ & .17 & .35 & .03 & .00 & .00 & .00 & .00 \\
\hline 22 & $\cdots$ & $\cdots$ & $\ldots$ & $\cdots$ & $\cdots$ & .15 & .30 & .03 & .00 & .00 & .00 & .00 \\
\hline 23 & $\cdots$ & $\cdots$ & $\ldots$ & $\cdots$ & $\ldots$ & .12 & .31 & .05 & .00 & .00 & .00 & .00 \\
\hline 24 & $\cdots$ & $\cdots$ & $\cdots$ & $\cdots$ & $\cdots$ & .10 & .26 & .05 & .00 & .00 & .00 & .00 \\
\hline 25 & $\cdots$ & $\cdots$ & $\cdots$ & $\cdots$ & $\cdots$ & .11 & .80 & .08 & .00 & .00 & .00 & .00 \\
\hline 26 & $\cdots$ & $\cdots$ & $\ldots$ & $\ldots$ & $\ldots$ & .14 & .84 & .17 & .00 & .00 & .00 & .00 \\
\hline 27 & $\cdots$ & $\cdots$ & $\cdots$ & $\cdots$ & $\ldots$ & .19 & .67 & .09 & .00 & .00 & .00 & .00 \\
\hline 28 & $\cdots$ & $\cdots$ & $\cdots$ & $\cdots$ & $\cdots$ & .25 & .85 & .07 & .00 & .00 & .00 & .00 \\
\hline 29 & $\cdots$ & $\cdots$ & $\ldots$ & $\cdots$ & $\ldots$ & .55 & 1.4 & .09 & .00 & .00 & .00 & .00 \\
\hline 30 & $\cdots$ & $\cdots$ & $\cdots$ & $\cdots$ & $\ldots$ & .67 & 2.5 & .02 & .00 & .00 & .00 & .00 \\
\hline 31 & $\cdots$ & $\cdots$ & $\cdots$ & $\cdots$ & $\cdots$ & .87 & $\cdots$ & .01 & $\cdots$ & .00 & .00 & $\cdots$ \\
\hline TOTAL & $\ldots$ & $\cdots$ & $\cdots$ & $\ldots$ & $\cdots$ & $\ldots$ & 20.75 & 11.92 & 0.19 & 0.00 & 0.00 & 0.00 \\
\hline MEAN & $\cdots$ & $\cdots$ & $\cdots$ & $\cdots$ & $\cdots$ & $\cdots$ & .69 & .38 & .006 & .000 & .000 & .000 \\
\hline MAX & $\cdots$ & $\cdots$ & $\cdots$ & $\ldots$ & $\cdots$ & $\cdots$ & 2.3 & 1.6 & .07 & .00 & .00 & .00 \\
\hline MIN & $\cdots$ & $\cdots$ & $\cdots$ & $\cdots$ & $\cdots$ & $\cdots$ & .26 & .01 & .00 & .00 & .00 & .00 \\
\hline$A C \cdot F T$ & $\cdots$ & $\cdots$ & - . & $\cdots$ & $\cdots$ & $\cdots$ & 41 & 24 & .4 & .00 & .00 & .00 \\
\hline
\end{tabular}


SITE NUMBER. - - GS14

STATION IDENTIFICATION. - 395304105105100

LOCATION. - Lat $39^{\circ} 53^{\prime} 04^{\prime \prime}$, long $105^{\circ} 10^{\prime} 51^{\prime \prime}$, in $\mathrm{NW}^{1} / 4 \mathrm{NW}^{1} / 4 \mathrm{sec} .13, \mathrm{~T} .2 \mathrm{~S} ., \mathrm{R} .70$ w., Jefferson County, Hydrologic Unit 10190003, Rocky Flats Environmental Technology Site, between Woman Creek Diversion and Mower Diversion.

DRAINAGE AREA. - - $1.4 \mathrm{mi}^{2}$.

PERIOD OF RECORD. - March 1994 to current year.

GAGE. - Water-stage recorder and Parshall flume with weir plate. Elevation of gage is $5,705 \mathrm{ft}$ above sea level.

REMARKS. - Records poor.

DISCHARGE, CUBIC FEET PER SECOND, WATER YEAR OCTOBER 1994 'TO SEPTEMBER 1995

\begin{tabular}{|c|c|c|c|c|c|c|c|c|c|c|c|c|}
\hline DAY & $O C T$ & NOV & DEC & JAN & ГEB & MAR & $A P R$ & MAY & JUN & JUL & $A \cup G$ & SEP \\
\hline 1 & .00 & .00 & .08 & e. 10 & .24 & e.12 & .29 & e 3.0 & 2.4 & .61 & 2.3 & .00 \\
\hline 2 & .00 & .00 & .12 & e.00 & .13 & e. 10 & .18 & e2.5 & 2.1 & .41 & .19 & .00 \\
\hline 3 & .00 & $.0 n$ & e. 12 & e. 00 & .13 & e. 13 & .14 & 2.8 & 3.6 & .28 & .03 & .00 \\
\hline 4 & .00 & .00 & e. 11 & e. 00 & .12 & e. 14 & .11 & 2.9 & 2.8 & .22 & .00 & .00 \\
\hline 5 & .00 & .00 & e. 10 & e. 00 & $\therefore 1$ & e. 13 & .09 & 1.5 & 2.7 & .19 & .00 & .00 \\
\hline 6 & .00 & .08 & e. 10 & e. 00 & .10 & e. 15 & e. 00 & 1.2 & 1.4 & .12 & .00 & .00 \\
\hline 7 & .00 & .05 & e. 10 & e. 00 & .11 & e. 14 & e. 00 & .85 & 1.0 & .09 & .00 & .00 \\
\hline 8 & .00 & .05 & e. 10 & e. 00 & $\therefore 1$ & e. 22 & e. 00 & .87 & 2.7 & .11 & .00 & .00 \\
\hline 9 & .00 & .12 & e. 00 & e. 13 & .12 & .36 & e. 00 & .82 & 4.9 & .09 & .00 & .00 \\
\hline 10 & .00 & .19 & e. 00 & e. 20 & e. 10 & .34 & e. 14 & .59 & 2.9 & .08 & .00 & .00 \\
\hline 11 & .00 & .13 & e. 00 & e. 17 & e. 10 & .11 & e. 25 & .53 & 2.3 & .05 & .00 & .00 \\
\hline 12 & .00 & .08 & e. 00 & e. 14 & e. 00 & .09 & e. 70 & .54 & 1.3 & .02 & .00 & .00 \\
\hline 13 & .00 & .05 & e. 00 & e. 12 & e. 00 & .06 & e. 23 & .40 & .95 & .00 & .00 & .00 \\
\hline 14 & .00 & .11 & e. 00 & e. 10 & e. 10 & .05 & e. 10 & .41 & .74 & .06 & .00 & .00 \\
\hline 15 & .00 & .13 & e. 00 & e. 12 & e. 22 & .04 & e. 00 & .41 & .58 & .14 & .00 & .00 \\
\hline 16 & .00 & .15 & e. 00 & e. 10 & e. 17 & .05 & e. 00 & .36 & .42 & .08 & .00 & .00 \\
\hline 17 & .00 & .11 & e. 00 & e. 10 & e. 25 & .08 & e. 25 & e 20 & .41 & .07 & .00 & .00 \\
\hline 18 & .00 & .05 & e. 10 & e. 00 & e. 32 & .08 & e. 50 & 3.6 & .73 & .05 & .00 & .00 \\
\hline 19 & .00 & .04 & e. 10 & e. 00 & e. 25 & .06 & el.3 & 2.7 & .43 & .08 & .00 & .00 \\
\hline 20 & .00 & .08 & e. 10 & e. 00 & e. 20 & .04 & e2.9 & 1.8 & .32 & .15 & .00 & .00 \\
\hline 21 & .00 & .07 & e. 10 & .02 & e. 15 & .04 & e 3.2 & 1.4 & .25 & .06 & .00 & .00 \\
\hline 22 & .00 & .08 & e. 10 & .00 & e. 14 & .03 & e3.9 & 1.1 & .24 & .02 & .00 & .00 \\
\hline 23 & .00 & .07 & e. 12 & .04 & e. 13 & .02 & e3.8 & 2.9 & .21 & .00 & .00 & .00 \\
\hline 24 & .00 & .11 & e. 13 & .01 & e. 12 & .03 & e4. 2 & 4.4 & .30 & .00 & .00 & .00 \\
\hline 25 & .00 & .11 & e. 15 & .02 & e. 11 & .02 & e 3.5 & 3.4 & .28 & .00 & .00 & .00 \\
\hline 26 & .00 & .11 & e. 17 & .05 & e. 10 & .04 & e4. 8 & 2.9 & .20 & .00 & .00 & .00 \\
\hline 27 & .00 & .04 & e. 15 & .10 & e. 10 & .09 & e 6.0 & 4.8 & .16 & .00 & .00 & .00 \\
\hline 28 & .00 & .01 & e. 13 & .10 & e. 11 & .14 & e4.0 & 3.8 & .32 & .00 & .00 & .00 \\
\hline 29 & .00 & .00 & e. 10 & .11 & $\ldots$ & .18 & e5.6 & 4.4 & 1.7 & 1.2 & .00 & .00 \\
\hline 30 & .00 & .02 & e. 10 & .03 & $\cdots$ & .18 & e5.0 & 3.7 & .88 & 3.7 & .00 & .00 \\
\hline 31 & .00 & $\cdots$ & e. 10 & .14 & $\cdots$ & .35 & .. & 2.8 & $\cdots$ & 3.5 & .00 & $\cdots$ \\
\hline TOTAL & 0.00 & 2.04 & 2.48 & 1.90 & 3.84 & 3.61 & 51.18 & 83.38 & 39.22 & 11.38 & 2.52 & 0.00 \\
\hline MEAN & .000 & .068 & .080 & .061 & .14 & .12 & 1.71 & 2.69 & 1.31 & .37 & .081 & .000 \\
\hline MAX & .00 & .19 & .17 & .20 & .32 & .36 & 6.0 & 20 & 4.9 & 3.7 & 2.3 & .00 \\
\hline MIN & .00 & .00 & .00 & .00 & .00 & .02 & .00 & .36 & .16 & .00 & .00 & .00 \\
\hline$A C-F T$ & .00 & 4.0 & 4.9 & 3.8 & 7.6 & 7.2 & 102 & 165 & 78 & 23 & 5.0 & .00 \\
\hline
\end{tabular}

STATISTICS OF MONTHLY MEAN DATA FOR WATER YEARS $1994 \cdot 1995$, BY WATER YEAR (WY)

$\begin{array}{llll}\text { MEAN } & .000 & .068 & .080 \\ \text { MAX } & .000 & .068 & .080 \\ \text { (WY) } & 1995 & 1995 & 1995 \\ \text { MIN } & .000 & .068 & .080 \\ \text { (WY) } & 1995 & 1995 & 1995\end{array}$

SUMMARY STATISTICS

ANNUAL, TOTAL

ANNUAL MEAN

HIGHEST ANNUAL, MEAN

LOWEST ANNUAL MEAN

HIGHEST DAILY MEAN

LOWEST DAILY MEAN

ANNUAL, SEVEN-DAY MINIMUM

ANNUAL RUNOFF (AC-FT)

10 PERCENT EXCEEDS

50 PERCENT EXCEEDS

90 PERCENT EXCEEDS

.061
.061
1995
.061
1995

.14
.24
1995
.14
1995
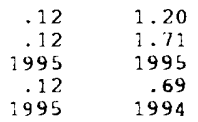

1.54
2.69
1995
.38
1994

FOR 1995 WATER YEAR

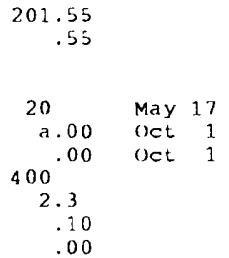

$\begin{array}{rrrr}.66 & .18 & .041 & .000 \\ 1.31 & .37 & .081 & .000 \\ 1995 & 1995 & 1995 & 1994 \\ .006 & .000 & .000 & .000 \\ 1994 & 1994 & 1994 & 1994\end{array}$

WATER YEARS 1994 - 1995

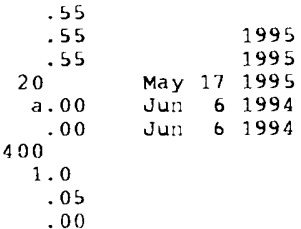

a No flow many days

e Estimated 
SMART DITCH ABOVE POND D-1

SITE NUMBER. - - GS15

STATION IDENTIFICATION. - 395246105111800

LOCATION. - Lat $39^{\circ} 52^{\prime} 46^{\prime \prime}$, long $105^{\circ} 11^{\prime} 13^{\prime \prime}$, in $\mathrm{SE}^{1} / 4 \mathrm{NE}^{1} / 4$ sec. 14, T. 2 s., R.70 W., Jefferson County, Hydrologic Unit 10190003, Rocky Flats Environmental Technology site, at concrete splitter box that routes flow to D-series ponds.

DRAINAGE AREA. - - $1.18 \mathrm{mi}^{2}$.

PERIOD OF RECORD. - - March 1994 to current year.

GAGE. - Water-stage recorder and Parshall flume with weir plate. Elevation of gage is $5,757 \mathrm{ft}$ above sea level.

REMARKS. - Records fair except for estımated daily discharges, which are poor. No data July 5-18 due to equipment failure.

DISCHARge, CUBIC FEET' PER SECOND, WATER YEAR OCTOBER 1993 TO SEPTEMBER 1994 DAIISY MEAN VALUES

\begin{tabular}{|c|c|c|c|c|c|c|c|c|c|c|c|c|}
\hline DAY & $\varkappa_{\mathrm{CT}}$ & Nov & $\mathrm{DE}, \mathrm{C}$ & JAN & $F E B$ & MAR & $A P R$ & MAY & JUN & JUI, & AUG & SEP \\
\hline 1 & $\ldots$ & $\ldots$ & $\ldots$ & $\ldots$ & $\ldots$ & $\ldots$ & .03 & .10 & .06 & .08 & .00 & .00 \\
\hline 2 & $\ldots$ & $\cdots$ & $\ldots$ & $\ldots$ & $\ldots$ & $\cdots$ & .01 & .09 & .06 & .09 & .00 & .00 \\
\hline 3 & $\cdots$ & $\cdots$ & $\cdots$ & $\cdots$ & $\cdots$ & $\ldots$ & .00 & .07 & .08 & .09 & .00 & .00 \\
\hline 4 & $\cdots$ & $\cdots$ & $\cdots$ & $\cdots$ & $\cdots$ & $\ldots$ & .00 & .05 & .08 & .09 & .00 & .00 \\
\hline is & $\cdots$ & $\cdots$ & $\ldots$ & $\cdots$ & $\ldots$ & $\ldots$ & .02 & .03 & .06 & $\therefore$ & .00 & .00 \\
\hline 6 & $\cdots$ & $\cdots$ & $\ldots$ & $\ldots$ & $\cdots$ & $\ldots$ & .04 & .02 & .16 & $\cdots$ & .00 & .00 \\
\hline 7 & $\cdots$ & $\ldots$ & $\cdots$ & $\cdots$ & $\cdots$ & $\ldots$ & .02 & .02 & .36 & $\cdots$ & .00 & .00 \\
\hline 8 & $\cdots$ & $\ldots$ & $\cdots$ & $\ldots$ & $\cdots$ & $\ldots$ & .00 & .01 & .51 & $\ldots$ & .00 & .00 \\
\hline 9 & $\cdots$ & $\ldots$ & $\cdots$ & $\cdots$ & $\cdots$ & $\ldots$ & .02 & .00 & .71 & $\cdots$ & .00 & .00 \\
\hline 10 & $\cdots$ & $\cdots$ & $\cdots$ & $\cdots$ & $\cdots$ & $\cdots$ & .06 & .01 & .71 & $\cdots$ & .00 & .00 \\
\hline 11 & $\cdots$ & $\cdots$ & $\ldots$ & $\ldots$ & $\ldots$ & $\ldots$ & .07 & .00 & .72 & $\ldots$ & .00 & .00 \\
\hline 12 & $\cdots$ & $\cdots$ & $\cdots$ & $\cdots$ & $\cdots$ & $\cdots$ & .26 & .00 & .64 & $\cdots$ & .00 & .00 \\
\hline 13 & $\cdots$ & $\ldots$ & $\ldots$ & $\ldots$ & $\cdots$ & $\ldots$ & .16 & .03 & .35 & $\ldots$ & .00 & .00 \\
\hline 14 & $\ldots$ & $\ldots$ & $\cdots$ & $\cdots$ & $\cdots$ & $\cdots$ & .09 & .05 & .27 & $\ldots$ & .00 & .00 \\
\hline 15 & $\ldots$ & $\cdots$ & $\cdots$ & $\ldots$ & $\cdots$ & $\ldots$ & .05 & .00 & .21 & $\ldots$ & .00 & .00 \\
\hline 16 & $\cdots$ & $\ldots$ & $\ldots$ & $\ldots$ & $\ldots$ & $\ldots$ & .02 & .00 & .22 & $\ldots$ & .00 & .00 \\
\hline 17 & $\ldots$ & $\ldots$ & $\ldots$ & $\ldots$ & $\ldots$ & $\ldots$ & .01 & .00 & .22 & $\cdots$ & .00 & .00 \\
\hline 18 & $\cdots$ & $\cdots$ & $\cdots$ & $\cdots$ & $\cdots$ & $\cdots$ & .00 & .00 & .21 & $\ldots$ & .00 & .00 \\
\hline 19 & $\cdots$ & $\cdots$ & $\cdots$ & $\cdots$ & $\cdots$ & $\cdots$ & .00 & .00 & .18 & .15 & .00 & .00 \\
\hline 20 & $\cdots$ & $\cdots$ & $\cdots$ & $\cdots$ & $\cdots$ & $\cdots$ & .00 & .00 & .17 & .09 & .00 & .00 \\
\hline 21 & $\ldots$ & $\cdots$ & $\ldots$ & $\ldots$ & $\ldots$ & $\cdots$ & .00 & .00 & .17 & .01 & .00 & .00 \\
\hline 22 & $\cdots$ & $\cdots$ & $\ldots$ & $\cdots$ & $\cdots$ & $\cdots$ & .00 & .01 & .15 & .00 & .00 & .00 \\
\hline 23 & $\cdots$ & $\ldots$ & $\ldots$ & $\ldots$ & $\ldots$ & $\ldots$ & .00 & .07 & .12 & .00 & .00 & .00 \\
\hline 24 & $\cdots$ & $\cdots$ & $\cdots$ & $\cdots$ & $\cdots$ & $\cdots$ & .00 & .06 & .11 & .00 & .00 & .00 \\
\hline 25 & $\ldots$ & $\cdots$ & $\cdots$ & $\cdots$ & $\cdots$ & $\cdots$ & .14 & .06 & .11 & .00 & .00 & .00 \\
\hline 26 & $\ldots$ & $\cdots$ & $\cdots$ & $\ldots$ & $\cdots$ & . - & .06 & .07 & .10 & e.00 & .00 & .00 \\
\hline 27 & $\ldots$ & $\cdots$ & $\cdots$ & $\cdots$ & $\cdots$ & $\ldots$ & .06 & .06 & .08 & $\begin{array}{r}.00 \\
\end{array}$ & .00 & .00 \\
\hline 28 & $\cdots$ & $\cdots$ & $\cdots$ & $\cdots$ & $\cdots$ & $\ldots$ & .09 & .08 & .08 & .00 & .00 & $\begin{array}{l}.00 \\
.00\end{array}$ \\
\hline 29 & $\ldots$ & $\ldots$ & $\cdots$ & $\ldots$ & $\ldots$ & $\ldots$ & .22 & .05 & .09 & .00 & .00 & .00 \\
\hline 30 & $\ldots$ & $\cdots$ & $\ldots$ & $\ldots$ & $\cdots$ & $\ldots$ & .29 & .03 & .08 & .00 & .00 & .00 \\
\hline 31 & $\ldots$ & $\ldots$ & $\ldots$ & $\ldots$ & $\cdots$ & .06 & $\therefore$ & .06 & & .00 & .00 & \\
\hline TOTAL & $\cdots$ & $\ldots$ & $\cdots$ & $\ldots$ & $\ldots$ & $\ldots$ & 1.72 & 1.03 & 7.07 & $\ldots$ & 0.00 & 0.00 \\
\hline MEAN & & $\ldots$ & $\ldots$ & $\ldots$ & $\ldots$ & $\ldots$ & .057 & .033 & .24 & $\ldots$ & .000 & .000 \\
\hline MAX & $\ldots$ & $\ldots$ & $\ldots$ & $\ldots$ & $\ldots$ & $\ldots$ & .29 & .10 & .72 & $\ldots$ & .00 & .00 \\
\hline MIN & $\ldots$ & $\ldots$ & $\ldots$ & $\ldots$ & $\ldots$ & $\ldots$ & .00 & .00 & .06 & $\ldots$ & .00 & .00 \\
\hline$A C-F T$ & & $\ldots$ & $\cdots$ & $\cdots$ & $\cdots$ & $\ldots$ & $\begin{array}{l}-00 \\
3.4\end{array}$ & 20 & $\begin{array}{r}.06 \\
14\end{array}$ & $\ldots$ & .00 & .00 \\
\hline
\end{tabular}

e Estimated 
SITE NUMBER. - - GS15

STATION IDENTIFICATION. - 395246105111800

LOCATION. - - Lat $39^{\circ} 52^{\prime} 46^{\prime \prime}$, long $105^{\circ} 11^{\prime} 18^{\prime \prime}$, in $\mathrm{SE}^{1} / 4 \mathrm{NE}^{1} / 4 \mathrm{sec} .14$, T.2 S., R.70 W., Jefferson County, Hydrologic Unit 10190003, Rocky Flats Environmental Technology Site, at concrete splitter box that routes flow to $D$-series ponds.

DRAINAGE AREA. - - $1.18 \mathrm{mi}^{2}$.

PERIOD OF RECORD. - March 1994 to MaY 1y95. Station discontinued June 1, 1995.

GAGE. - Water-stage recorder and Parshall flume with weir plate. Elevation of gage is $5,757 \mathrm{ft}$ above sea level.

REMARKS. - Records poor.

DISCHARGE, CUBIC PFE'T PER SECOND, WATER YEAR OCTOBER 1994 TOO SEPTEMBER 1996

\begin{tabular}{|c|c|c|c|c|c|c|c|c|c|c|c|c|}
\hline DAY & $x^{\prime} \mathrm{T}$ & NOV & DEC & JAN & FEB & MAR & $A P R$ & MAY & JUN & JUL & $A \cup G$ & SEP \\
\hline 1 & .00 & .00 & .00 & .00 & .09 & .00 & .42 & .29 & $\cdots$ & $\cdots$ & $\cdots$ & $\cdots$ \\
\hline 2 & .00 & .00 & .00 & .00 & .00 & .00 & .24 & .15 & $\cdots$ & $\cdots$ & $\cdots$ & $\cdots$ \\
\hline 3 & .00 & .00 & .00 & .00 & .06 & .07 & .05 & e. 15 & $\cdots$ & $\cdots$ & $\cdots$ & $\cdots$ \\
\hline 4 & .00 & .00 & .00 & .00 &.$\$ 3$ & .00 & .00 & e. 20 & $\cdots$ & $\cdots$ & $\cdots$ & $\cdots$ \\
\hline 5 & .00 & .00 & .00 & .00 & .09 & .00 & .00 & .18 & $\cdots$ & $\cdots$ & $\cdots$ & $\cdots$ \\
\hline 6 & .00 & .00 & .00 & .00 & .05 & .02 & .00 & .11 & $\cdots$ & $\cdots$ & $\cdots$ & $\cdots$ \\
\hline 7 & .00 & .00 & .00 & .00 & .00 & e. 05 & .00 & .07 & $\ldots$ & $\cdots$ & $\cdots$ & $\cdots$ \\
\hline 8 & .00 & .00 & .00 & .00 & .01 & e. 00 & .00 & .07 & $\cdots$ & $\cdots$ & $\cdots$ & $\cdots$ \\
\hline 9 & .00 & .00 & .00 & .00 & .02 & .69 & .00 & .04 & $\cdots$ & $\cdots$ & $\cdots$ & $\cdots$ \\
\hline 10 & .00 & .00 & .00 & .00 & .00 & .69 & .00 & .03 & $\cdots$ & $\cdots$ & $\cdots$ & $\cdots$ \\
\hline 11 & .00 & .00 & .00 & .00 & .00 & .63 & .16 & .03 & $\cdots$ & $\cdots$ & $\cdots$ & $\cdots$ \\
\hline 12 & .00 & .00 & .00 & .00 & .00 & .29 & .59 & .02 & $\cdots$ & $\cdots$ & $\cdots$ & \\
\hline 13 & .00 & .00 & .00 & .00 & .00 & .01 & .74 & .01 & $\cdots$ & $\cdots$ & $\cdots$ & $\cdots$ \\
\hline 14 & .00 & .00 & .00 & .00 & .26 & .00 & .59 & .01 & $\ldots$ & $\cdots$ & $\cdots$ & $\cdots$ \\
\hline 15 & .00 & .00 & .00 & .00 & .12 & .00 & .47 & .00 & $\cdots$ & $\cdots$ & $\cdots$ & $\cdots$ \\
\hline 16 & .00 & .00 & .00 & .00 & .04 & .00 & .22 & .02 & $\cdots$ & $\cdots$ & $\cdots$ & $\cdots$ \\
\hline 17 & .00 & .00 & .00 & .00 & .08 & .00 & .72 & 1.1 & $\cdots$ & $\cdots$ & $\cdots$ & \\
\hline 18 & .00 & .00 & .00 & .00 & .45 & .00 & .73 & .27 & $\cdots$ & $\cdots$ & $\cdots$ & $\cdots$ \\
\hline 19 & .00 & .00 & .00 & .00 & .68 & .00 & .95 & .01 & $\cdots$ & $\cdots$ & $\cdots$ & $\cdots$ \\
\hline 20 & .00 & .00 & .00 & .00 & .65 & .00 & .88 & .05 & $\cdots$ & $\cdots$ & $\cdots$ & $\cdots$ \\
\hline 21 & .00 & .00 & .00 & .00 & .64 & .00 & .89 & .12 & $\cdots$ & $\cdots$ & $\cdots$ & $\cdots$ \\
\hline 22 & .00 & .00 & .00 & .00 & .59 & .00 & 1.0 & .21 & $\cdots$ & $\cdots$ & $\cdots$ & $\cdots$ \\
\hline 23 & .00 & .00 & .00 & .00 & .52 & .00 & .92 & .60 & $\cdots$ & $\cdots$ & $\cdots$ & $\cdots$ \\
\hline 24 & .00 & .00 & .00 & .00 & .48 & .00 & .82 & 1.4 & $\cdots$ & $\cdots$ & $\cdots$ & $\cdots$ \\
\hline 25 & .00 & .00 & .00 & .00 & .30 & .00 & .85 & .76 & $\cdots$ & $\cdots$ & $\cdots$ & \\
\hline 26 & .00 & .00 & .00 & .00 & .02 & .00 & .77 & .56 & $\cdots$ & $\cdots$ & $\cdots$ & \\
\hline 27 & .00 & .00 & .00 & .00 & .00 & .00 & 1.0 & 1.5 & $\cdots$ & $\cdots$ & $\cdots$ & $\cdots$ \\
\hline 28 & .00 & .00 & .00 & .00 & .00 & .00 & .95 & 1.0 & $\cdots$ & $\cdots$ & $\cdots$ & $\cdots$ \\
\hline 29 & .00 & .00 & .00 & .00 & $\cdots$ & .00 & .97 & 2.5 & $\cdots$ & $\cdots$ & $\cdots$ & - \\
\hline 30 & .00 & .00 & .00 & .00 & $\cdots$ & .09 & .79 & 2.8 & $\cdots$ & $\cdots$ & $\cdots$ & $\cdots$ \\
\hline 31 & .00 & $\cdots$ & .00 & .03 & $\cdots$ & .32 & $\cdots$ & 1.6 & $\cdots$ & $\cdots$ & $\cdots$ & \\
\hline TOTAL, & 0.00 & 0.00 & 0.00 & 0.03 & 5.28 & 2.86 & 15.72 & 15.86 & $\cdots$ & $\cdots$ & $\cdots$ & \\
\hline MEAN & .000 & .000 & .000 & .001 & .19 & .092 & .52 & .51 & $\cdots$ & $\cdots$ & $\cdots$ & \\
\hline MAX & .00 & .00 & .00 & .03 & .68 & .69 & 1.0 & 2.8 & $\cdots$ & $\cdots$ & $\cdots$ & $\cdots$ \\
\hline MIN & .00 & .00 & .00 & .00 & .00 & .00 & .00 & .00 & $\cdots$ & $\cdots$ & $\cdots$ & \\
\hline$A C \cdot F T$ & .00 & .00 & .00 & .06 & 10 & 5.7 & 31 & 31 & $\cdots$ & $\cdots$ & $\cdots$ & \\
\hline
\end{tabular}

STATISTICS OF MONTHLY MEAN DATA FOR WATER YEARS 1994 - 1995, BY WATER YEAR (WY)

\begin{tabular}{|c|c|c|c|c|c|c|c|c|c|c|c|c|}
\hline MEAN & .000 & .000 & .000 & .001 & .19 & .092 & .29 & .27 & .24 & $\cdots$ & .000 & .000 \\
\hline $\operatorname{MAX}$ & .000 & .000 & .000 & $.00 \%$ & $\therefore 9$ & .092 & .52 & .51 & .24 & $\cdots$ & .000 & .000 \\
\hline (WY) & 1995 & 1995 & 1995 & 1995 & 1995 & 1995 & 1995 & 1995 & 1994 & $\cdots$ & 1994 & 1994 \\
\hline MIN & .000 & .000 & .000 & $.00 i$ & .19 & .092 & .057 & .033 & .24 & $\cdots$ & .000 & .000 \\
\hline (WY) & 1995 & 1995 & 1995 & 1995 & 1995 & 1995 & 1994 & 1994 & 1994 & . . & 1994 & 1994 \\
\hline
\end{tabular}

SUMMARY STATISTICS

HIGHEST DALLY MEAN LOWEST DAILY MEAN ANNUAL SEVEN-DAY MINIMUM 10 PERCENT EXCEEDS 50 PERCENT EXCEEDS 90 PERCENT EXCEEDS

a No flow many days

e Estimated
WATER YEARS 1994 - 1995

$$
\begin{array}{llrl}
2.8 & \text { May } & 30 & 1995 \\
9.00 & \text { Apr } & 3 & 1994 \\
.00 & \text { Apr } & 18 & 1994 \\
.48 & & & \\
.00 & & & \\
.00 & & &
\end{array}
$$


ANTELOPE SPRINGS CREEK ABOVE WOMAN CREEK

SITE NUMBER. - - GS16

STATION IDENTIFICATION. - 395301105120800

LOCATION. - Lat $39^{\circ} 53^{\prime} 01^{\prime \prime}$, Iong $105^{\circ} 12^{\prime} 08^{\prime \prime}$, in $\mathrm{NW}^{1} / 4 \mathrm{NW}^{1} / 4$ sec. $14, \mathrm{~T} .2 \mathrm{~S} ., \mathrm{R} .70$ W., Jefferson County, Hydrologic Unit 10190003, Rocky Flats Environmental Technology site, 750 feet upstream from the confluence with Woman creek.

DRAINAGE AREA. $-0.21 \mathrm{mi}^{2}$.

PERIOD OF RECORD. - March 1994 to current year.

GAGE. - Water-stage recorder and Parshall flume with weir plate. Elevation of gage is $5,900 \mathrm{ft}$ above sea level.

REMARKS. - - Records poor.

DISCHARGE, CUBIC FEET PER SFCOND, WATFR YFAR OCTOBER 1993 TU SEPTEMBFR 1994

\begin{tabular}{|c|c|c|c|c|c|c|c|c|c|c|c|c|}
\hline DAY & $O C T$ & $\mathrm{NOV}$ & DEC & JAN & FFB & MAR & $A P R$ & MAY & JUN & JUI, & $A \cup G$ & SEP \\
\hline 1 & $\cdots$ & $\cdots$ & $\cdots$ & $\cdots$ & $\cdots$ & $\cdots$ & .11 & .17 & .05 & .01 & .01 & e. 01 \\
\hline 2 & $\cdots$ & $\cdots$ & $\cdots$ & $\cdots$ & $\cdots$ & $\cdots$ & .11 & .21 & .04 & .01 & .01 & e. 01 \\
\hline 3 & $\cdots$ & $\cdots$ & $\cdots$ & $\ldots$ & $\cdots$ & . . & .09 & .15 & .05 & .01 & e. 01 & e. 01 \\
\hline 4 & $\ldots$ & $\cdots$ & $\ldots$ & $\cdots$ & $\cdots$ & .. & .07 & .12 & .05 & .01 & e. 01 & e. 01 \\
\hline 5 & $\cdots$ & $\cdots$ & $\cdots$ & $\cdots$ & $\cdots$ & $\cdots$ & e. 20 & .10 & .04 & .01 & e. 01 & e. 01 \\
\hline 6 & $\cdots$ & $\cdots$ & $\cdots$ & $\cdots$ & $\cdots$ & $\cdots$ & e. 16 & .09 & .03 & .01 & e. 01 & e. 01 \\
\hline 7 & $\cdots$ & $\cdots$ & $\cdots$ & $\cdots$ & $\cdots$ & $\cdots$ & e. 14 & .09 & .03 & .01 & e. 01 & e. 01 \\
\hline 8 & $\cdots$ & $\cdots$ & $\cdots$ & $\cdots$ & $\cdots$ & $\cdots$ & e. 12 & .07 & .03 & .01 & e. 01 & e. 01 \\
\hline 9 & $\cdots$ & $\cdots$ & $\cdots$ & $\cdots$ & $\cdots$ & $\cdots$ & e. 09 & .08 & .02 & .01 & e.01 & e. 01 \\
\hline 10 & $\cdots$ & $\cdots$ & $\cdots$ & $\cdots$ & $\cdots$ & $\cdots$ & e. 11 & .09 & .02 & .01 & e. 02 & e. 01 \\
\hline 11 & $\cdots$ & $\cdots$ & $\cdots$ & $\cdots$ & $\cdots$ & $\cdots$ & e. 80 & .07 & .02 & .01 & e.01 & e. 01 \\
\hline 12 & $\cdots$ & $\cdots$ & $\cdots$ & $\cdots$ & $\cdots$ & $\cdots$ & e. 40 & .07 & .02 & .01 & e.01 & e. 01 \\
\hline 13 & $\cdots$ & $\cdots$ & $\cdots$ & $\cdots$ & $\cdots$ & $\cdots$ & e. 09 & .17 & .02 & .01 & e. 01 & e. 01 \\
\hline 14 & $\cdots$ & $\cdots$ & $\cdots$ & $\cdots$ & $\cdots$ & $\cdots$ & e. 06 & .11 & .02 & .02 & e. 01 & e. 01 \\
\hline 15 & $\cdots$ & $\cdots$ & $\cdots$ & $\cdots$ & $\cdots$ & .07 & e. 06 & .06 & .02 & .02 & e. 01 & e. 01 \\
\hline 16 & $\cdots$ & $\cdots$ & $\cdots$ & $\cdots$ & $\cdots$ & .08 & e. 06 & .04 & .02 & .01 & e. 01 & e. 01 \\
\hline 17 & $\cdots$ & $\cdots$ & $\cdots$ & $\cdots$ & $\cdots$ & .06 & e. 06 & .03 & .02 & .01 & e. 01 & e. 01 \\
\hline 18 & $\cdots$ & $\cdots$ & $\cdots$ & $\cdots$ & $\cdots$ & .07 & e. 06 & .04 & .02 & .01 & e. 01 & e. 01 \\
\hline 19 & $\cdots$ & $\cdots$ & $\cdots$ & $\cdots$ & $\cdots$ & .05 & e. 06 & .04 & .02 & .01 & e. 01 & e. 01 \\
\hline 20 & $\cdots$ & $\cdots$ & $\cdots$ & $\cdots$ & $\cdots$ & .07 & e. 07 & .04 & .02 & .02 & e.01 & e.01 \\
\hline 21 & $\cdots$ & $\cdots$ & $\cdots$ & $\cdots$ & $\cdots$ & .07 & e. 08 & .04 & .02 & .01 & e. 01 & .02 \\
\hline 22 & $\cdots$ & $\ldots$ & $\cdots$ & $\cdots$ & $\cdots$ & .06 & e. 09 & .03 & .03 & .01 & e. 01 & .04 \\
\hline 23 & $\cdots$ & $\cdots$ & $\cdots$ & $\cdots$ & $\cdots$ & .05 & e. 10 & .03 & .02 & .01 & e. 01 & .02 \\
\hline 24 & $\cdots$ & $\cdots$ & $\cdots$ & $\cdots$ & $\cdots$ & .06 & e. 35 & .04 & .02 & .01 & e. 01 & .02 \\
\hline 25 & $\cdots$ & $\cdots$ & $\cdots$ & $\cdots$ & $\cdots$ & .07 & e. 74 & .04 & .01 & .01 & e. 01 & .02 \\
\hline 26 & $\cdots$ & $\cdots$ & $\cdots$ & $\cdots$ & $\cdots$ & .08 & e. 14 & .05 & .01 & .01 & e. 01 & .01 \\
\hline 27 & $\cdots$ & $\cdots$ & $\cdots$ & $\cdots$ & $\cdots$ & .10 & .14 & .04 & .01 & .01 & e.01 & .01 \\
\hline 28 & $\cdots$ & $\cdots$ & $\cdots$ & $\cdots$ & $\cdots$ & .15 & .20 & .06 & .01 & .01 & e. 01 & .01 \\
\hline 29 & $\cdots$ & $\cdots$ & $\cdots$ & $\cdots$ & $\cdots$ & .15 & .25 & .05 & .01 & .01 & e.01 & .01 \\
\hline 30 & $\cdots$ & $\cdots$ & $\cdots$ & $\cdots$ & $\cdots$ & .26 & .42 & .03 & .01 & .01 & e. 01 & .02 \\
\hline 31 & $\cdots$ & $\cdots$ & $\cdots$ & $\cdots$ & $\cdots$ & .17 & $\cdots$ & .05 & $\cdots$ & .01 & e. 01 & $\cdots$ \\
\hline TOTAL & $\cdots$ & $\cdots$ & $\cdots$ & $\cdots$ & $\cdots$ & $\cdots$ & 5.43 & 2.30 & 0.71 & 0.34 & 0.32 & 0.38 \\
\hline MEAN & $\cdots$ & $\cdots$ & $\cdots$ & $\cdots$ & $\cdots$ & $\cdots$ & .18 & .074 & .024 & .011 & .010 & .013 \\
\hline MAX & $\cdots$ & $\cdots$ & $\cdots$ & $\cdots$ & $\cdots$ & $\cdots$ & .80 & .21 & .05 & .02 & .02 & .04 \\
\hline MIN & $\cdots$ & $\cdots$ & $\cdots$ & $\cdots$ & $\cdots$ & $\cdots$ & .06 & .03 & .01 & .01 & .01 & .01 \\
\hline$A C-F T$ & $\cdots$ & $\cdots$ & $\cdots$ & $\cdots$ & $\cdots$ & $\cdots$ & 11 & 4.6 & 1.4 & .7 & .6 & .8 \\
\hline
\end{tabular}

e Estimated 
ANTELOPE SPRINGS CREEK ABOVE WOMAN CREEK

SITE NUMBER. - - GS16

STATION IDENTIFICATION. - - 395301105120800

LOCATION. - Lat $39^{\circ} 53^{\prime} 01^{\prime \prime}$, long $105^{\circ} 12^{\prime} 08^{\prime \prime}$, in $\mathrm{NN}^{1} / 4 \mathrm{NW}^{1} / 4$ sec. $14, \mathrm{~T} .2 \mathrm{~S} ., \mathrm{R} .70 \mathrm{~W}$. , Iefferson County, Hydrologic unit 10190003, Rocky Flats Environmental Technology site, 750 feet upstream from the confluence with Woman creek.

DRAINAGE AREA. - - $0.21 \mathrm{mi}^{2}$.

PERIOD OF RECORD.--March 1994 to current year.

GAGE. - Water-stage recorder and Parshall flume with weir plate. Elevation of gage is 5,900 ft above sea level.

REMARKS. - Records poor.

DISCHARGE, CUBIC FEET PER SECOND, WATER YEAR OCTOBER 1994 TO SEPTEMBER 1995

\begin{tabular}{|c|c|c|c|c|c|c|c|c|c|c|c|c|}
\hline DAY & $(\mathbb{C T}$ & NOV & DEC & JAN & FEB & MAR & APR & MAY & JUN & JUI, & $A \cup G$ & SEP \\
\hline 1 & .03 & .04 & e. 10 & e. 03 & e. 14 & e. 05 & .06 & .23 & e. 40 & .08 & .01 & .01 \\
\hline 2 & .02 & .05 & e. 09 & e. 03 & e. 08 & e. 05 & .04 & .24 & e. 29 & .05 & .01 & .01 \\
\hline 3 & .02 & .07 & e. 07 & e.03 & e. 07 & e. 05 & .04 & .33 & .93 & .04 & .01 & .01 \\
\hline 4 & .03 & .09 & e. 08 & e. 03 & e. 06 & e. 06 & .04 & .24 & .66 & .03 & .01 & .01 \\
\hline 5 & .03 & .16 & e. 06 & e. 04 & e. 06 & e. 06 & .04 & .18 & .36 & .03 & .01 & .01 \\
\hline 6 & .03 & .08 & e. 06 & e.07 & e. 05 & e. 10 & .04 & .13 & .19 & .02 & .01 & .01 \\
\hline 7 & .03 & .06 & e. 06 & e. 12 & e. 05 & e. 15 & .06 & .11 & .16 & .02 & .01 & .02 \\
\hline 8 & .03 & .08 & e. 06 & e. 20 & e.05 & e. 20 & .05 & .13 & .98 & .02 & .01 & .02 \\
\hline 9 & .03 & .13 & e. 06 & e. 15 & e.05 & .20 & .07 & .11 & 3.7 & .02 & .01 & .02 \\
\hline 10 & .03 & .11 & e. 06 & e. 11 & e. 05 & .08 & .07 & .09 & .31 & .01 & .01 & .02 \\
\hline 11 & .03 & .07 & e. 06 & e. 14 & e. 05 & .06 & .18 & .10 & .22 & .01 & .01 & .02 \\
\hline 12 & .03 & .06 & e.06 & e. 05 & e. 05 & .04 & .35 & .10 & .15 & .01 & .01 & .01 \\
\hline 13 & .04 & .06 & e. 04 & e. 15 & e. 05 & .04 & .14 & .07 & .12 & .01 & .01 & .01 \\
\hline 14 & .05 & .08 & e. 02 & e.11 & e. 24 & .04 & .13 & .10 & .11 & .02 & .01 & .01 \\
\hline 15 & .06 & .07 & e. 00 & e. 05 & e. 32 & .05 & .11 & .08 & .10 & .02 & .01 & .01 \\
\hline 16 & .07 & .08 & e. 01 & e.06 & e. 15 & .07 & .28 & .05 & .09 & .02 & .01 & .01 \\
\hline 37 & .16 & .07 & e.01 & e.06 & e.06 & .09 & .62 & e6.3 & .14 & .02 & .01 & .01 \\
\hline 18 & .07 & .06 & e. 01 & e. 05 & e. 05 & .07 & .51 & e. 60 & .13 & .02 & .01 & .02 \\
\hline 19 & .07 & .00 & e. 01 & e.05 & e. 05 & .09 & .40 & e. 47 & .09 & .04 & .02 & .04 \\
\hline 20 & .07 & .04 & e. 03 & e. 05 & e. 06 & .10 & .53 & e. 30 & .05 & .03 & .01 & .05 \\
\hline 2.1 & .06 & .07 & e.06 & e.05 & e. 06 & .06 & .47 & e. 24 & .03 & .02 & .01 & .05 \\
\hline 22 & .06 & .06 & e. 15 & e.06 & e. 06 & .04 & .62 & e. 20 & .03 & .01 & .01 & .12 \\
\hline 23 & .06 & .08 & e. 08 & e. 06 & e. 06 & .03 & .78 & .16 & .03 & .01 & .01 & .04 \\
\hline 24 & .06 & .08 & e. 16 & e.05 & e. 05 & .02 & .98 & .54 & .05 & .01 & .01 & .04 \\
\hline 25 & .06 & .08 & e. 12 & e.11 & e.05 & .02 & .44 & .13 & .04 & .01 & .01 & .03 \\
\hline 26 & .06 & .09 & e.09 & e. 10 & c. 05 & .07 & .52 & .06 & .03 & .01 & .01 & .04 \\
\hline 27 & .05 & .02 & e. 06 & e. 11 & e. 05 & .06 & 1.3 & .67 & .03 & .01 & .01 & .03 \\
\hline 28 & .05 & e. 07 & e. 05 & e. 08 & e.05 & .06 & .51 & .34 & .18 & .01 & .01 & .03 \\
\hline 29 & .05 & e. 09 & e. 03 & e. 06 & $\cdots$ & .16 & 1.1 & 1.4 & .37 & .01 & .01 & .04 \\
\hline 30 & .06 & e. 12 & e. 03 & e.06 & $\cdots$ & .14 & .97 & 1.7 & .10 & .01 & .01 & .08 \\
\hline 31 & .05 & $\cdots$ & e. 03 & e. 22 & $\cdots$ & .12 & $\cdots$ & e. 10 & $\cdots$ & .01 & .01 & $\cdots$ \\
\hline TOTAL & 1.55 & 2.22 & 1.81 & 2.54 & 2.17 & 2.43 & 11.45 & 15.50 & 10.07 & 0.64 & 0.32 & 0.83 \\
\hline MEAN & .050 & .074 & .058 & .082 & .077 & .078 & .38 & .50 & .34 & .021 & .010 & .028 \\
\hline MAX & .16 & .16 & .16 & .22 & .32 & .20 & 1.3 & 6.3 & 3.7 & .08 & .02 & .12 \\
\hline MIN & .02 & .00 & .00 & .03 & .05 & .02 & .04 & .05 & .03 & .01 & .01 & .01 \\
\hline $\mathrm{AC} \cdot \mathrm{FT}$ & 3.1 & 4.4 & 3.6 & 5.0 & $4 \cdot 3$ & 4.8 & 23 & 31 & 20 & 1.3 & .6 & 1.6 \\
\hline
\end{tabular}

STATISTICS (IF MONTHLY MIAN DATA FOR WATER YEARS 1994 - 1996, BY WATER YEAR (WY)

\begin{tabular}{|c|c|c|c|c|c|c|c|c|c|c|c|c|c|c|}
\hline MEAN & .050 & .074 & .058 & .082 & .077 & \multicolumn{2}{|r|}{.078} & .28 & .29 & .18 & .016 & & .010 & .020 \\
\hline $\operatorname{MAX}$ & .050 & .074 & .058 & .082 & .077 & & .078 & .38 & .50 & .34 & .021 & & .010 & .028 \\
\hline (WY) & 1995 & 1995 & 1995 & 1995 & 1995 & & 1995 & 1995 & 1995 & 1995 & 1995 & & 1994 & 1995 \\
\hline$M I N$ & .050 & .074 & .058 & .082 & .077 & & .078 & .18 & .074 & .024 & .011 & & .010 & .013 \\
\hline (WY) & 1995 & 1995 & 1995 & 1995 & 1995 & & 1995 & 1994 & 1994 & 1994 & 1994 & & 1994 & 1994 \\
\hline SUMMA & ARY STATIS & & & & FOR & 1995 & WATER & YEAR & & & WATER & YEARS & S 1994 & 1995 \\
\hline ANNUA & AL TOTAL & & & & & 51.5 & & & & & & & & \\
\hline ANNUA & AL MEAN & & & & & & 14 & & & & & 14 & & \\
\hline $\mathrm{HIGHE}$ & EST ANNUAI & AAN & & & & & & & & & & 14 & & 1995 \\
\hline LOWES & ST ANNUAI, & & & & & & & & & & & 14 & & 1995 \\
\hline $\mathrm{HIGHE}$ & EST DAILY & & & & & 6.3 & $\cdot M_{i}$ & y 17 & & & 6 & 3 & May 17 & 1995 \\
\hline LOWES & ST DAILY $M$ & & & & & a. 00 & Noy & 19 & & & b. & & Nov 191 & 1994 \\
\hline ANNUA & AL SEVEN-D & MI N I MU: & & & & .0 & $\mathrm{~J}$ & 1122 & & & & 01 & Jun 25 & 1994 \\
\hline ANNUA & AL RUNOFE & $(-F T)$ & & & & 102 & & & & & 102 & & & \\
\hline $10 \mathrm{PE}$ & ERCENT EXC & & & & & & 26 & & & & & 20 & & \\
\hline $50 \mathrm{PE}$ & ERCENT EXC & & & & & & 06 & & & & & 05 & & \\
\hline $90 \mathrm{PE}$ & ERCENT EXC & & & & & & 01 & & & & & 01 & & \\
\hline
\end{tabular}

\footnotetext{
a Also Occurred Dec, 15

b No flow at times some years

e Estimated
} 
SITE NUMBER. - - GS17

STATION IDENTIFICATION. - 395309105114100

LOCATION. - Lat $39^{\circ} 53^{\prime} 09^{\prime \prime}$, long $105^{\circ} 11^{\prime} 41^{\prime \prime}$, in $\mathrm{SE}^{1} / 4 \mathrm{SW}^{1} / 4 \mathrm{sec} .11$, T. 2 S., R. 70 W., Jefferson County, Hydrologic Unit 10190003, Rocky Flats Environmental Tecinnology Site, 500 feet upstream from Pond $\mathrm{C} \cdot 1$, and 1,500 feet downstream from the Antelope springs confluence.

DRAINAGE AREA. - - $1.17 \mathrm{mi}^{2}$.

PERIOD OF RECORD. - -March 1994 to current year.

GAGE. - Water-stage recorder and Parshall flume with weir plate. Elevation of gage is $5,860 \mathrm{ft}$ above sea level.

REMARKS.--Records fair except for estimated daily discharges, which are poor. Discharge, CUBIC FHET PER SECOND, WATER YeAR OCTOBER 2993 TO SEPTEMBER 1994

\begin{tabular}{|c|c|c|c|c|c|c|c|c|c|c|c|c|}
\hline DAY & $\mathrm{MCT}$ & NOV & DEC & JAN & FEB & MAR & APR & MAY & JUN & JUI, & AUG & SEF \\
\hline : & $\ldots$ & $\ldots$ & $\cdots$ & $\ldots$ & $\ldots$ & $\cdots$ & e. 26 & $\therefore 2$ & .12 & .00 & .00 & .01 \\
\hline 2 & $\cdots$ & $\cdots$ & $\cdots$ & $\cdots$ & $\cdots$ & $\cdots$ & e. 26 & $\therefore 4$ & .08 & .00 & .00 & .01 \\
\hline 3 & $\cdots$ & $\cdots$ & $\cdots$ & $\cdots$ & $\cdots$ & $\cdots$ & e. 25 & $i .1$ & .13 & .00 & .00 & .01 \\
\hline 4 & $\cdots$ & $\ldots$ & $\cdots$ & $\ldots$ & $\ldots$ & .58 & e. 25 & .88 & .14 & .00 & .00 & .00 \\
\hline 5 & $\cdots$ & $\cdots$ & $\cdots$ & $\cdots$ & $\cdots$ & .49 & e. 26 & .71 & .07 & .00 & .00 & .00 \\
\hline 6 & $\cdots$ & $\cdots$ & $\cdots$ & $\ldots$ & $\ldots$ & .55 & e. 27 & .64 & .05 & .00 & .00 & .00 \\
\hline 7 & $\cdots$ & $\ldots$ & $\cdots$ & $\cdots$ & $\cdots$ & .66 & e. 26 & .60 & .03 & .00 & .00 & .00 \\
\hline 8 & $\cdots$ & $\cdots$ & $\cdots$ & $\cdots$ & $\cdots$ & .49 & .27 & .50 & .02 & .00 & .00 & .00 \\
\hline 9 & $\ldots$ & $\ldots$ & $\cdots$ & $\ldots$ & $\cdots$ & .72 & .26 & .50 & .02 & .00 & .00 & .00 \\
\hline 10 & $\cdots$ & $\ldots$ & $\ldots$ & $\cdots$ & $\cdots$ & .57 & .27 & .53 & .02 & .00 & .00 & .00 \\
\hline 11 & $\cdots$ & $\cdots$ & $\cdots$ & $\ldots$ & $\cdots$ & .45 & .27 & .42 & $.0 b$ & .00 & .00 & .00 \\
\hline 17 & $\cdots$ & $\cdots$ & $\cdots$ & $\cdots$ & $\cdots$ & .43 & .28 & .39 & .05 & .00 & .00 & .00 \\
\hline 13 & $\cdots$ & $\cdots$ & $\cdots$ & $\cdots$ & $\cdots$ & .36 & .27 & .59 & .02 & .00 & .00 & .00 \\
\hline 14 & $\cdots$ & $\cdots$ & $\cdots$ & $\cdots$ & $\ldots$ & .33 & .27 & .54 & .01 & .00 & .00 & .00 \\
\hline 15 & $\cdots$ & $\cdots$ & $\cdots$ & $\cdots$ & $\cdots$ & .29 & .27 & .33 & .01 & .00 & .00 & .00 \\
\hline 16 & $\cdots$ & $\cdots$ & $\cdots$ & $\cdots$ & $\cdots$ & .28 & .27 & .24 & .01 & .00 & .00 & .01 \\
\hline 17 & $\cdots$ & $\cdots$ & $\cdots$ & $\cdots$ & $\cdots$ & .25 & .27 & .19 & .02 & .00 & .00 & .00 \\
\hline 18 & $\cdots$ & $\cdots$ & $\cdots$ & $\cdots$ & $\cdots$ & .23 & .27 & .15 & .02 & .00 & .00 & .00 \\
\hline 19 & $\cdots$ & $\cdots$ & $\cdots$ & $\cdots$ & $\ldots$ & .21 & .27 & .13 & .02 & .00 & .00 & .00 \\
\hline 20 & $\cdots$ & $\cdots$ & $\cdots$ & $\cdots$ & $\cdots$ & .23 & .34 & .12 & .01 & .00 & .00 & .00 \\
\hline 21 & $\ldots$ & $\ldots$ & $\ldots$ & $\ldots$ & $\ldots$ & .22 & .35 & .14 & .01 & .00 & .00 & .02 \\
\hline 22 & $\ldots$ & $\ldots$ & $\cdots$ & $\cdots$ & $\cdots$ & .20 & .34 & .17 & .02 & .00 & .00 & .03 \\
\hline 23 & $\ldots$ & $\ldots$ & $\cdots$ & $\ldots$ & $\ldots$ & .17 & .33 & .15 & .01 & .00 & .00 & .02 \\
\hline 24 & $\cdots$ & $\cdots$ & $\cdots$ & $\cdots$ & $\cdots$ & .18 & .27 & .15 & .01 & .00 & .00 & .02 \\
\hline 25 & $\cdots$ & $\cdots$ & $\cdots$ & $\cdots$ & $\cdots$ & .19 & 1.1 & .23 & .00 & .00 & .00 & .01 \\
\hline 26 & $\ldots$ & $\ldots$ & $\ldots$ & $\ldots$ & $\ldots$ & .21 & .63 & .23 & .00 & .00 & .00 & .01 \\
\hline 27 & $\cdots$ & $\cdots$ & $\cdots$ & $\ldots$ & $\ldots$ & .24 & .72 & .17 & .00 & .00 & .00 & .01 \\
\hline 28 & $\cdots$ & $\ldots$ & $\cdots$ & $\ldots$ & $\cdots$ & e. 26 & .89 & .22 & .00 & .00 & .00 & .01 \\
\hline 29 & $\cdots$ & $\cdots$ & $\cdots$ & $\ldots$ & $\cdots$ & e. 27 & 1.6 & .14 & .00 & .00 & .00 & .01 \\
\hline 30 & $\ldots$ & $\ldots$ & $\ldots$ & $\cdots$ & $\cdots$ & e. 28 & 2.5 & .08 & .00 & .00 & .00 & .02 \\
\hline 31 & $\cdots$ & $\cdots$ & $\cdots$ & $\cdots$ & $\cdots$ & e. 27 & & .11 & $\cdots$ & .00 & .01 & \\
\hline TOTAL & $\ldots$ & $\ldots$ & $\ldots$ & . . & $\ldots$ & $\cdots$ & 14.12 & 12.95 & 0.95 & 0.00 & 0.01 & 0.20 \\
\hline MEAN & $\cdots$ & $\cdots$ & $\cdots$ & $\cdots$ & $\cdots$ & $\ldots$ & .47 & .42 & .032 & .000 & .000 & .007 \\
\hline $\operatorname{MAX}$ & $\cdots$ & $\ldots$ & & . & $\cdots$ & . & 2.5 & 1.4 & .14 & .00 & .01 & .03 \\
\hline MIN & $\cdots$ & . & $\cdots$ & $\cdots$ & $\cdots$ & $\cdots$ & .25 & .08 & .00 & .00 & .00 & .00 \\
\hline$A C \cdot F T$ & $\cdots$ & $\cdots$ & & $\cdots$ & $\cdots$ & $\ldots$ & 28 & 26 & 1.9 & .00 & .02 & \\
\hline
\end{tabular}

e Estimated 
LOCATION. - Lat $39^{\circ} 53^{\prime} 09^{\prime \prime}$, long $105^{\circ} 11^{\prime} 41^{\prime \prime}$, in $\mathrm{SE}^{1} / 4 \mathrm{SW}^{1} / 4 \mathrm{sec} .11, \mathrm{~T} .2 \mathrm{~S} ., \mathrm{R} .70 \mathrm{~W}$., Jefferson County, Hydrologic Unit 10190003, Rocky Flats Environmental Technology Site, 500 feet upstream from Pond $\mathrm{C}-1$, and 1,500 feet downstream from the Antelope Springs confluence.

DRATNAGE AREA. - - $1.17 \mathrm{mi}^{2}$.

PERIOD OF RECORD.--March 1994 to May 1995. Station discontinued May 20, 1995.

GAGE. - Water-stage recorder and Parshall flume with weir plate. Elevation of gage is $5,860 \mathrm{ft}$ above sea level.

REMARKS. - Records fair except tor estimated daily discharges, which are poor. DISCHARGE, CUBiC FEET PER SECOND, WATER YEAR OCTOBER 1994 'TO SEPTEMBER $199 \mathrm{~b}$

\begin{tabular}{|c|c|c|c|c|c|c|c|c|c|c|c|c|}
\hline DAY & (xT & NOV & DEC & JAN & FEB & MAR & A PR & MAY & JUN & JUL, & AUG & SEP \\
\hline 1 & .03 & .07 & .19 & e. 10 & .42 & .14 & .27 & e2. 5 & $\ldots$ & $\ldots$ & $\ldots$ & $\cdots$ \\
\hline 2 & .03 & .08 & .15 & e.00 & .24 & .16 & .21 & e2.1 & $\cdots$ & $\cdots$ & $\cdots$ & $\cdots$ \\
\hline 3 & .02 & .12 & .13 & e.00 & .18 & .26 & .19 & e2.7 & $\cdots$ & $\cdots$ & $\cdots$ & $\cdots$ \\
\hline 4 & .03 & .15 & .13 & e.00 & .17 & .20 & .16 & e 2.4 & $\cdots$ & $\cdots$ & $\cdots$ & $\cdots$ \\
\hline 5 & .03 & .24 & .12 & e.00 & .16 & .17 & .15 & 1.4 & $\cdots$ & $\cdots$ & $\cdots$ & $\cdots$ \\
\hline 6 & .03 & .17 & .13 & e.00 & .16 & .19 & .14 & 1.1 & $\cdots$ & $\cdots$ & $\cdots$ & $\cdots$ \\
\hline 7 & .03 & .13 & .13 & e. 14 & .15 & .20 & .13 & .85 & $\cdots$ & $\cdots$ & $\ldots$ & \\
\hline 8 & .03 & .16 & .12 & e. 23 & .15 & .35 & .11 & .87 & $\cdots$ & $\cdots$ & $\cdots$ & - \\
\hline 9 & .03 & .27 & .04 & e. 29 & .16 & .44 & .14 & .70 & $\cdots$ & $\cdots$ & $\cdots$ & \\
\hline 10 & .03 & .25 & .00 & e.33 & e.15 & .25 & .19 & .59 & $\cdots$ & $\cdots$ & $\cdots$ & $\cdots$ \\
\hline 11 & .03 & .16 & e. 02 & e. 29 & e. 14 & .19 & .34 & .57 & $\cdots$ & $\cdots$ & $\cdots$ & $\cdots$ \\
\hline 12 & .02 & .16 & e. 00 & e. 26 & e. 13 & .15 & 1.1 & .53 & $\cdots$ & $\cdots$ & $\cdots$ & \\
\hline 13 & .03 & .13 & e. 02 & e. 24 & e. 12 & .14 & .54 & .40 & $\cdots$ & $\cdots$ & $\cdots$ & \\
\hline 14 & .03 & .20 & $\begin{array}{l}.10 \\
\end{array}$ & e.22 & e. 30 & .13 & .28 & .51 & $\ldots$ & $\cdots$ & $\cdots$ & $\cdots$ \\
\hline 15 & .04 & .11 & e. 07 & e.23 & .29 & .14 & .20 & .40 & $\cdots$ & $\cdots$ & $\cdots$ & $\cdots$ \\
\hline 16 & .05 & .25 & .14 & e. 21 & .28 & .14 & .23 & e. 60 & $\cdots$ & $\cdots$ & $\cdots$ & $\cdots$ \\
\hline 17 & .73 & .20 & .18 & e.18 & .32 & .19 & 1.1 & e60 & $\cdots$ & $\cdots$ & $\cdots$ & \\
\hline 18 & .74 & .15 & .19 & .15 & .51 & .15 & 1.3 & e 1.0 & $\cdots$ & $\cdots$ & $\cdots$ & \\
\hline 19 & .05 & .15 & e. 21 & .11 & .35 & .13 & e2.b & e1.9 & $\cdots$ & $\cdots$ & $\cdots$ & \\
\hline 20 & .05 & .21 & e. 23 & .12 & .25 & .13 & e2.7 & $\cdots$ & $\cdots$ & $\cdots$ & $\cdots$ & \\
\hline 21 & .04 & .17 & e. 24 & .08 & .21 & .11 & e3.0 & $\cdots$ & $\cdots$ & $\cdots$ & $\cdots$ & $\cdots$ \\
\hline 22 & .04 & .15 & e. 25 & e.00 & .19 & .10 & e3.2 & $\ldots$ & $\ldots$ & $\cdots$ & $\cdots$ & \\
\hline 23 & .05 & .17 & e. 24 & e.00 & .17 & .11 & e3.1 & $\cdots$ & $\cdots$ & $\cdots$ & $\cdots$ & - \\
\hline 24 & .05 & .19 & e. 25 & e.00 & .16 & .10 & e3. 3 & $\cdots$ & $\cdots$ & $\cdots$ & $\cdots$ & \\
\hline 25 & .05 & .19 & e. 26 & e.13 & .15 & .11 & e2.7 & $\cdots$ & $\cdots$ & $\cdots$ & $\cdots$ & \\
\hline $2 \epsilon$ & .05 & .17 & e. 27 & e.19 & .14 & .18 & e 4.4 & $\cdots$ & $\cdots$ & $\cdots$ & $\cdots$ & $\cdot$ \\
\hline 27 & .05 & .11 & e. 25 & e. 22 & .15 & .20 & e 5.2 & $\cdots$ & $\cdots$ & $\cdots$ & $\cdots$ & \\
\hline 28 & .05 & .09 & e. 23 & .17 & .15 & .25 & e2. .9 & $\cdots$ & $\cdots$ & $\cdots$ & $\cdots$ & \\
\hline 29 & .07 & .07 & e. 21 & .19 & $\cdots$ & .25 & e4.9 & $\cdots$ & $\cdots$ & $\cdots$ & $\cdots$ & - \\
\hline 30 & .07 & .13 & e. 16 & .20 & $\cdots$ & .38 & e4.4 & $\cdots$ & $\cdots$ & $\cdots$ & $\cdots$ & \\
\hline 31 & .08 & & e. 13 & .34 & $\cdots$ & .41 & & & & $\cdots$ & $\cdots$ & \\
\hline TOTAL & 2.66 & 4.80 & 4.79 & 4.62 & 5.95 & 6.05 & 49.08 & $\cdots$ & $\cdots$ & $\cdots$ & $\cdots$ & \\
\hline MEAN & .086 & .16 & .15 & .15 & .21 & .20 & 1.64 & $\cdots$ & $\cdots$ & $\cdots$ & $\cdots$ & \\
\hline MAX & .74 & .27 & .27 & .34 & .51 & .44 & 5.2 & $\cdots$ & $\cdots$ & . & $\cdots$ & \\
\hline MIN & .02 & .07 & .00 & .00 & .12 & .10 & .11 & $\cdots$ & & & $\cdots$ & \\
\hline$A C-F T$ & 5.3 & 9.5 & 9.5 & 9.2 & 12 & 12 & & $\cdots$ & $\cdots$ & $\ldots$ & $\cdots$ & \\
\hline
\end{tabular}

STATISTICS OF MONTHLY MEAN DATA FOR WATER YEARS 1994 - 1995, BY WATER YEAR (WY)

\begin{tabular}{|c|c|c|c|c|c|c|c|c|c|c|c|c|}
\hline MEAN & .086 & .16 & ל & .15 & .21 & .20 & 1.05 & .42 & .032 & .000 & .000 & .007 \\
\hline $\operatorname{MAX}$ & .086 & $\therefore 16$ & . 11 & .15 & .21 & .20 & 1.64 & .42 & .032 & .000 & 000 & 007 \\
\hline (WY) & 1995 & 1995 & 1995 & 1995 & 1995 & 1995 & 1995 & 1994 & 1994 & 1994 & 1994 & 1994 \\
\hline MIN & .086 & .16 & .15 & .15 & .21 & .20 & .47 & .42 & .032 & .000 & .000 & .007 \\
\hline$(W Y)$ & 1995 & 1995 & 1995 & 1995 & 1995 & 1995 & 1994 & 1994 & 1994 & 1994 & 1994 & 1994 \\
\hline
\end{tabular}

SUMMARY STATISTICS

HIGHEST DAILY MEAN LOWEST DAILY MEAN

ANNUAL SEVEN-DAY MINIMUM

10 PERCENT EXCEEDS

50 PERCENT EXCEEDS

90 PERCENT EXCEEDS

a No flow many days

e Estimated
WATER YEARS 1994 - 1995

$\begin{array}{rlr}60 & \text { May } 17 & 1995 \\ \text { a.00 } & \text { Jun } 25 & 1994 \\ .00 & \text { Jun } 25 & 1994 \\ .60 & & \\ .15 & \end{array}$


WOMAN CREEK ABOVE OLD LANDFILL

SITE NUMBER. - - GS18

STATION IDENTIFICATION. - 395308105123100

LOCATION. - Lat $39^{\circ} 52^{\prime} 08^{\prime \prime}$, long $105^{\circ} 12^{\prime} 31^{\prime \prime}$, in $\mathrm{NW}^{1} / 4 \mathrm{NE}^{1 / 4} \mathrm{sec} .15, \mathrm{~T} .2$ S., R. 70 W., Jefferson

County, Hydrologic Unit 10190003, Rocky Flats Environmental Technology Site, 500 feet upstream from the old landfill, and 1,750 feet downstream from the confluence of North and South woman Creeks.

DRAINAGE AREA. $-0.78 \mathrm{mi}^{2}$.

PERIOD OF RECORD. - March 1994 to current year.

GAGE. - Water-stage recorder and Parshall flume with weir plate. Elevation of gage is $5,953 \mathrm{ft}$ above sea level.

REMARKS.- Records fair.

DISCHARGE, CUBIC FEET PER SECONJ, WATER YEAR OCTUBER 1993 T'U SEPTEMBER 1994

\begin{tabular}{|c|c|c|c|c|c|c|c|c|c|c|c|c|}
\hline DAY & ()CT & $\mathrm{NOSV}$ & DEC & JAN & FE3 & MAR & $A P R$ & MAY & JUN & JUI, & $A \cup G$ & SEP \\
\hline 1 & $\cdots$ & $\cdots$ & $\cdots$ & $\cdots$ & $\cdots$ & $\cdots$ & .22 & .86 & .02 & .00 & .00 & .00 \\
\hline 2 & $\cdots$ & $\ldots$ & $\ldots$ & $\ldots$ & $\ldots$ & $\ldots$ & .19 & .99 & .02 & .00 & .00 & .00 \\
\hline 3 & $\cdots$ & $\cdots$ & $\cdots$ & $\cdots$ & $\cdots$ & $\cdots$ & .15 & .77 & .03 & .00 & .00 & .00 \\
\hline 4 & $\cdots$ & $\ldots$ & $\ldots$ & $\cdots$ & $\ldots$ & $\ldots$ & .11 & .64 & .04 & .00 & .00 & .00 \\
\hline 5 & $\cdots$ & $\cdots$ & $\cdots$ & $\cdots$ & $\cdots$ & $\cdots$ & .17 & .51 & .02 & .00 & .00 & .00 \\
\hline 6 & $\cdots$ & $\cdots$ & $\ldots$ & $\cdots$ & $\ldots$ & $\ldots$ & .28 & .43 & .01 & .00 & .00 & .00 \\
\hline 7 & $\cdots$ & $\ldots$ & $\ldots$ & $\cdots$ & $\ldots$ & $\ldots$ & .20 & .37 & .01 & .00 & .00 & .00 \\
\hline 8 & $\cdots$ & $\cdots$ & $\ldots$ & $\cdots$ & $\cdots$ & $\cdots$ & .12 & .30 & .00 & .00 & .00 & .00 \\
\hline 9 & $\cdots$ & $\cdots$ & $\ldots$ & $\cdots$ & $\ldots$ & $\cdots$ & .17 & .29 & .00 & .00 & .00 & .00 \\
\hline 10 & $\cdots$ & $\cdots$ & $\cdots$ & $\cdots$ & $\cdots$ & $\cdots$ & .40 & .31 & .01 & .00 & .00 & .00 \\
\hline 11 & $\cdots$ & $\cdots$ & $\ldots$ & $\cdots$ & $\cdots$ & $\cdots$ & .46 & .23 & .07 & .00 & .00 & .00 \\
\hline 12 & $\cdots$ & $\ldots$ & $\ldots$ & $\cdots$ & $\ldots$ & $\ldots$ & 1.3 & .21 & .04 & .00 & .00 & .00 \\
\hline 13 & $\ldots$ & $\cdots$ & $\cdots$ & $\cdots$ & $\cdots$ & $\cdots$ & 1.2 & .25 & .02 & .00 & .00 & .00 \\
\hline 14 & $\cdots$ & $\cdots$ & $\cdots$ & $\cdots$ & $\cdots$ & $\cdots$ & .60 & .25 & .01 & .00 & .00 & .00 \\
\hline 15 & $\cdots$ & $\cdots$ & $\cdots$ & $\cdots$ & $\cdots$ & $\cdots$ & .43 & .13 & .00 & .00 & .00 & .00 \\
\hline 16 & $\cdots$ & $\ldots$ & $\ldots$ & $\ldots$ & $\ldots$ & . & .34 & .09 & .01 & .00 & .00 & .00 \\
\hline 17 & $\cdots$ & $\cdots$ & $\cdots$ & $\cdots$ & $\cdots$ & .05 & .31 & .06 & .04 & .00 & .00 & .00 \\
\hline 18 & $\cdots$ & $\cdots$ & $\ldots$ & $\cdots$ & $\ldots$ & .02 & .29 & .05 & .02 & .00 & .00 & .00 \\
\hline 19 & $\cdots$ & $\cdots$ & $\cdots$ & $\cdots$ & $\ldots$ & .01 & .28 & .04 & .01 & .00 & .00 & .00 \\
\hline 20 & $\cdots$ & $\cdots$ & $\ldots$ & $\cdots$ & $\cdots$ & .02 & .25 & .03 & .00 & .00 & .00 & .00 \\
\hline 21 & $\cdots$ & $\cdots$ & $\ldots$ & . . & $\ldots$ & .01 & .20 & .07 & .00 & .00 & .00 & .00 \\
\hline 22 & $\cdots$ & $\cdots$ & $\cdots$ & $\cdots$ & $\cdots$ & .00 & .19 & .09 & .00 & .00 & .00 & .00 \\
\hline 23 & $\ldots$ & $\cdots$ & $\cdots$ & $\cdots$ & $\cdots$ & .02 & .19 & .07 & .00 & .00 & .00 & .00 \\
\hline 24 & $\ldots$ & $\ldots$ & $\ldots$ & $\cdots$ & $\ldots$ & .01 & .14 & .09 & .00 & .00 & .00 & .00 \\
\hline 25 & $\cdots$ & $\cdots$ & $\cdots$ & $\ldots$ & $\cdots$ & .01 & .49 & .14 & .00 & .00 & .00 & .00 \\
\hline 26 & $\cdots$ & $\cdots$ & $\cdots$ & $\cdots$ & $\cdots$ & .04 & .35 & .11 & .00 & .00 & .00 & .00 \\
\hline 27 & $\ldots$ & $\ldots$ & $\ldots$ & $\cdots$ & $\cdots$ & .10 & .45 & .08 & .00 & .00 & .00 & .00 \\
\hline 28 & $\cdots$ & $\cdots$ & $\cdots$ & $\cdots$ & $\ldots$ & .20 & .52 & .10 & .00 & .00 & .00 & .00 \\
\hline 29 & $\cdots$ & $\cdots$ & $\cdots$ & $\cdots$ & $\ldots$ & .27 & 1.0 & .04 & .00 & .00 & .00 & .00 \\
\hline 30 & $\cdots$ & $\cdots$ & $\cdots$ & $\ldots$ & $\cdots$ & .47 & 1.9 & .02 & .00 & .00 & .00 & .00 \\
\hline 31 & $\cdots$ & $\cdots$ & $\cdots$ & $\cdots$ & $\cdots$ & .35 & $\cdots$ & .02 & $\ldots$ & .00 & .00 & $\cdots$ \\
\hline TOTAL & $\cdots$ & $\cdots$ & $\ldots$ & $\cdots$ & $\cdots$ & $\cdots$ & 17.90 & 7.64 & 0.38 & 0.00 & 0.00 & 0.00 \\
\hline MEAN & $\cdots$ & $\cdots$ & $\cdots$ & $\cdots$ & $\cdots$ & $\cdots$ & .43 & .25 & .013 & .000 & .000 & .000 \\
\hline $\operatorname{MAX}$ & $\cdots$ & $\cdots$ & $\cdots$ & $\cdots$ & $\cdots$ & $\cdots$ & 1.9 & .99 & .07 & .00 & .00 & .00 \\
\hline MIN & $\cdots$ & $\cdots$ & $\cdots$ & $\cdots$ & $\cdots$ & $\cdots$ & .11 & .02 & .00 & .00 & .00 & .00 \\
\hline$A C \cdot \mathrm{F}^{\prime} \mathrm{T}$ & $\cdots$ & $\cdots$ & $\cdots$ & $\cdots$ & $\ldots$ & $\cdots$ & 26 & 15 & .8 & .00 & .00 & .00 \\
\hline
\end{tabular}


SITE NUMBER. - - GS 18

STATION IDENTIFICATION. - 395308105123100

LOCATTON. - Lat $39^{\circ} 53^{\prime} 08^{\prime \prime}$, long $105^{\circ} 12^{\prime} 31^{\prime \prime}$, in $\mathrm{NW}^{1} / 4 \mathrm{NE}^{1} / 4$ sec. $15, \mathrm{~T} .2 \mathrm{~s} ., \mathrm{R} .70 \mathrm{~W} ., \mathrm{Jefferson}$ County, Hydrologic Unit 10190003, Rocky Flats Environmental Technology Site, 500 feet upstream from the old landfill, and 1,750 feet downstream from the confluence of North and South woman Creeks.

DRAINAGE AREA. - $0.78 \mathrm{mi}^{2}$.

PERIOD OF RECORD.--March 1994 to MaY 1995. Station discontinued May 3, 1995.

GAGE. - Water-stage recorder and Parshall flume with weir plate. Elevation of gage is 5,953 Et above sea level.

REMARKS. - Records poor.

DISCHARGE, CUBIC FEET PER SECOND, WATER YEAR OCTOBER : 994 TO SEPTEMBER 1995

\begin{tabular}{|c|c|c|c|c|c|c|c|c|c|c|c|c|c|}
\hline DAY & $\mathbb{M T}$ & NOS & DEC & & JAN & FEB & MAR & APR & MAY & JUN & JUL & AUG & SEP \\
\hline 1 & e. 04 & e. 00 & e. 0 s & & .00 & .00 & .03 & e. 03 & $\mathrm{e} 1.4$ & $\cdots$ & $\cdots$ & $\cdots$ & $\cdots$ \\
\hline 2. & e. 02 & e. 01 & e. 04 & & .00 & .03 & .03 & e. 03 & e1.4 & $\cdots$ & $\cdots$ & $\cdots$ & $\cdots$ \\
\hline 3 & e. 00 & e. 02 & e. 03 & & .00 & .05 & e. 02 & e. 03 & $\cdots$ & $\cdots$ & $\cdots$ & $\cdots$ & $\cdots$ \\
\hline 4 & e. 00 & e. 03 & e. 02 & & .00 & e.05 & .03 & e. 03 & $\ldots$ & $\cdots$ & $\ldots$ & $\cdots$ & $\cdots$ \\
\hline 5 & e. 00 & e. 04 & e. 02 & & .00 & e. 05 & .03 & .03 & $\cdots$ & $\cdots$ & $\cdots$ & $\cdots$ & $\cdots$ \\
\hline 6 & e. 00 & e. 02 & e. 02 & & .00 & .04 & .03 & .01 & $\cdots$ & $\cdots$ & $\cdots$ & $\cdots$ & $\cdots$ \\
\hline 7 & e. 00 & e. 01 & e. 0 ? & & .00 & .03 & .03 & .02 & $\cdots$ & $\cdots$ & $\cdots$ & $\cdots$ & $\cdots$ \\
\hline 8 & e. 00 & e. 03 & e. 02 & & .00 & .03 & e. 03 & .01 & $\ldots$ & $\cdots$ & $\ldots$ & $\cdots$ & $\ldots$ \\
\hline 9 & e. 00 & e. 06 & e. 02 & & .00 & .03 & e. 03 & .00 & $\cdots$ & $\cdots$ & $\cdots$ & $\cdots$ & $\cdots$ \\
\hline 10 & e. 00 & e. 04 & e. 02 & & .00 & .03 & e. 03 & .00 & $\cdots$ & $\cdots$ & $\cdots$ & $\cdots$ & $\cdots$ \\
\hline 11 & e. 00 & e. 04 & e. 01 & & .00 & .03 & e.03 & .00 & $\cdots$ & $\ldots$ & $\cdots$ & $\cdots$ & $\cdots$ \\
\hline 12 & e. 00 & e. 04 & .00 & & .00 & .03 & e. 03 & e. 22 & $\cdots$ & $\cdots$ & $\cdots$ & $\cdots$ & $\cdots$ \\
\hline 13 & e. 00 & e. 04 & .00 & & e.03 & .03 & e.03 & e.08 & $\ldots$ & $\cdots$ & $\ldots$ & $\cdots$ & $\cdots$ \\
\hline 14 & e. 00 & e. 08 & .00 & & .00 & .03 & e. 00 & e. 03 & $\ldots$ & $\cdots$ & $\cdots$ & $\cdots$ & $\cdots$ \\
\hline 15 & e. 00 & e. 19 & .00 & & .00 & .03 & e. 03 & e. 03 & $\cdots$ & $\cdots$ & $\cdots$ & $\cdots$ & $\cdots$ \\
\hline 16 & e. 02 & e. 06 & .00 & & .00 & .03 & e. 02 & e. 03 & $\cdots$ & $\cdots$ & $\cdots$ & $\cdots$ & $\cdots$ \\
\hline 17 & e. 08 & e. 05 & e. 02 & & e. 02 & e. 03 & e. 02 & e. 31 & $\ldots$ & $\cdots$ & $\cdots$ & $\cdots$ & $\cdots$ \\
\hline 18 & e. 02 & e. 02 & .00 & & .00 & e. 03 & e. 03 & e. 60 & $\cdots$ & $\ldots$ & $\cdots$ & $\cdots$ & $\cdots$ \\
\hline 19 & e. 00 & e. 00 & .00 & & .00 & e. 03 & e. 03 & e1.6 & $\ldots$ & $\cdots$ & $\cdots$ & $\cdots$ & $\ldots$ \\
\hline 20 & e. 00 & e. 02 & .00 & & .00 & e. 03 & e.03 & el. 3 & $\cdots$ & $\cdots$ & $\cdots$ & $\cdots$ & $\cdots$ \\
\hline 21 & e. 00 & e. 04 & .00 & & .00 & e. 03 & e. 03 & e1.9 & $\ldots$ & $\ldots$ & $\cdots$ & $\ldots$ & .. \\
\hline 22 & e. 00 & e. 02 & .00 & & .00 & e.03 & e. 03 & e 3.1 & $\ldots$ & $\ldots$ & $\cdots$ & $\cdots$ & $\cdots$ \\
\hline 23 & e. 00 & e. 02 & .00 & & .00 & e. 02 & e. 03 & e2. 8 & $\cdots$ & $\cdots$ & $\cdots$ & $\cdots$ & $\cdots$ \\
\hline 24 & e. 00 & e. 02 & e. 02 & & e. 02 & e. 02 & e. 03 & e 3.5 & $\cdots$ & $\cdots$ & $\cdots$ & $\cdots$ & $\cdots$ \\
\hline 25 & e. 00 & e. 02 & .00 & & .00 & e. 02 & .03 & $\mathrm{e} 2.4$ & $\cdots$ & $\cdots$ & $\cdots$ & $\cdots$ & $\cdots$ \\
\hline 26 & e. 00 & e. 02 & .00 & & .00 & e. 03 & .03 & e3.3 & $\cdots$ & $\cdots$ & $\cdots$ & $\cdots$ & $\cdots$ \\
\hline 27 & e. 00 & e. 02 & .00 & & .00 & e. 03 & .03 & e4.5 & $\cdots$ & $\cdots$ & $\cdots$ & $\cdots$ & $\cdots$ \\
\hline 28 & e. 00 & e. 02 & .00 & & .00 & .03 & .03 & $\mathrm{e} 3.2$ & $\ldots$ & $\cdots$ & $\cdots$ & $\cdots$ & $\cdots$ \\
\hline 29 & e. 00 & e.05 & .00 & & .00 & $\cdots$ & .03 & e4.0 & $\ldots$ & $\cdots$ & $\ldots$ & $\cdots$ & $\ldots$ \\
\hline 30 & e. 00 & e. 10 & .00 & & .00 & $\cdots$ & .03 & e4.1 & $\cdots$ & $\cdots$ & $\cdots$ & $\cdots$ & $\cdots$ \\
\hline 31 & e. 00 & $\ldots$ & .00 & & .00 & $\cdots$ & .03 & $\cdots$ & $\cdots$ & $\cdots$ & $\cdots$ & $\cdots$ & $\cdots$ \\
\hline T'OTAL & 0.18 & 1.13 & 0.21 & & 0.07 & 0.85 & 0.87 & 37.19 & $\cdots$ & $\cdots$ & $\cdots$ & $\cdots$ & $\cdots$ \\
\hline MEAN & .006 & .038 & .010 & & .002 & .030 & .028 & 1.24 & $\cdots$ & $\cdots$ & $\cdots$ & $\cdots$ & $\cdots$ \\
\hline MAX & .08 & .19 & .05 & & .03 & .05 & .03 & 4.5 & $\cdots$ & $\cdots$ & $\cdots$ & $\cdots$ & $\cdots$ \\
\hline MIN & .00 & .00 & .00 & & .00 & .00 & .00 & .00 & $\cdots$ & $\cdots$ & $\cdots$ & $\cdots$ & $\cdots$ \\
\hline$A C-F T$ & .4 & 2.2 & .6 & & .1 & 1.7 & 1.7 & 74 & $\cdots$ & $\cdots$ & $\cdots$ & $\cdots$ & $\cdots$ \\
\hline \multicolumn{2}{|c|}{ STATISTICS OF } & MONTHLY MEAN & DATA & For & WATER & YEARS 1994 & - 1995. & BY WA'TER & $Y \Xi A R \quad(W Y)$ & & & & \\
\hline MEAN & .006 & .038 & .010 & & .002 & .030 & .028 & .83 & .25 & .013 & .000 & .000 & .000 \\
\hline MAX & .006 & .038 & .010 & & .002 & .030 & .028 & 1.24 & .25 & .013 & .000 & .000 & .000 \\
\hline$(W Y)$ & 1995 & 1995 & 1995 & & 1995 & 1995 & 1995 & 1995 & i 994 & 1994 & 1994 & 1994 & 1994 \\
\hline MIN & .006 & .038 & .010 & & .002 & .030 & .028 & .43 & .25 & .013 & .000 & .000 & .000 \\
\hline$(W Y)$ & 1995 & 1995 & 1995 & & 1995 & 1995 & 1995 & 1994 & 1994 & 1994 & 1994 & 1994 & 1994 \\
\hline
\end{tabular}

SUMMARY STATISTICS

HIGHEST DAILY MEAN LOWEST DAILY MEAN ANNUAL SEVEN-DAY MINIMUM

10 PERCENT EXCEEDS

50 PERCENT EXCEEDS

90 PERCENT EXCEEISS

WATER YEARS $1994-1995$

a No flow many days

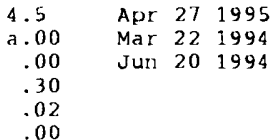

e Notimater 
SITE NUMBER. - - SW027

STATION IDENTIFICATION. - - 395313105110500

LOCATION. - Lat $39^{\circ} 53^{\prime} 12^{\prime \prime}$, long $105^{\circ} 11^{\prime} 04^{\prime \prime}$, in $\mathrm{SE}^{1} / 4 \mathrm{SE}^{1} / 4$ sec.11, T.2 S., R. 70 W., Jefferson County, Hydrologic unit 10190003, Rocky Flats Environmental Technology Site, at entrance to dual 66-inch corrugated metal culverts (CMPs) that convey water in the South Interceptor Ditch under woman Creek and into pond $\mathrm{C}-2$.

DRAINAGE AREA. - - $0.29 \mathrm{mi}^{2}$.

PERIOD OF RECORD. - October 1995 to current year. No daily-discharge data for 1994 water year; 1994 daily-stage data (for stage-activated water-quality sampling) reported by Rocky Mountain Remediation Services, L.L.C. (1995).

GAGE. - Water-stage recorder and 66-inch corrugated metal culverts with (starting April) V-notch weirs. Elevation of gage is $5,765 \mathrm{ft}$ above sea level.

REMARKS.--Records poor.

DISCHARGE, CUBIC FEET PER SECOND, WATER YEAR OCTYBER 1994 TO SEPTEMBER 1995

\begin{tabular}{|c|c|c|c|c|c|c|c|c|c|c|c|c|}
\hline DAY & $\mathscr{N T}$ & NoV & DEC & JAN & FEB & MAR & A PR & MAY & JUN & JUL, & $A \cup G$ & SEP \\
\hline 1 & .00 & .00 & e. 00 & .00 & e. 00 & .00 & .14 & .10 & .34 & .05 & .00 & .00 \\
\hline 2 & .00 & .00 & e. 00 & .00 & e. 00 & .00 & .11 & .03 & .37 & .02 & .00 & .00 \\
\hline 3 & .00 & .00 & e. 00 & .00 & e. 00 & .00 & .12 & .16 & .88 & .01 & .00 & .00 \\
\hline 4 & .00 & .00 & e. 00 & .00 & e. 00 & .00 & .08 & .22 & .51 & .01 & .00 & .00 \\
\hline 5 & .00 & .00 & e. 00 & .00 & e. 00 & .00 & .01 & .02 & .26 & .00 & .00 & .00 \\
\hline 6 & .00 & .03 & e. 00 & .00 & e. 00 & .00 & .00 & .01 & .09 & .00 & .00 & .00 \\
\hline 7 & .00 & .00 & e. 00 & .00 & .00 & .00 & .00 & .01 & .05 & .00 & .00 & .00 \\
\hline 8 & .00 & .00 & e. 00 & .00 & .00 & .01 & .00 & .01 & .72 & .00 & .00 & .00 \\
\hline 9 & .00 & .10 & e. 00 & .00 & .00 & .36 & .00 & .00 & 2.7 & .00 & .00 & .00 \\
\hline 10 & .00 & .07 & .00 & .00 & .00 & .14 & .00 & .00 & .26 & .00 & .00 & .00 \\
\hline 11 & .00 & .01 & .00 & .00 & .00 & .11 & .03 & .00 & .18 & .00 & .00 & .00 \\
\hline 12 & .00 & .00 & .00 & .00 & .00 & .08 & .21 & .00 & .10 & .00 & .00 & .00 \\
\hline 13 & .00 & .00 & .00 & e. 00 & .00 & .05 & .07 & .00 & .06 & .00 & .00 & .00 \\
\hline 14 & .00 & .00 & .00 & e. 00 & .00 & .00 & .01 & .00 & .04 & .00 & .00 & .00 \\
\hline 15 & .00 & e. 00 & .00 & e. 00 & .33 & .00 & .00 & .00 & .02 & .00 & .00 & .00 \\
\hline 16 & .00 & .01 & .00 & e. 00 & .28 & .00 & .00 & .00 & .02 & .00 & .00 & .00 \\
\hline 17 & .04 & .00 & .00 & e. 00 & .33 & .00 & .27 & e2.9 & .03 & .00 & .00 & .00 \\
\hline 18 & .06 & .00 & .00 & e. 00 & .42 & .00 & .23 & e. 70 & .23 & .00 & .00 & .00 \\
\hline 19 & .00 & e. 00 & .00 & e. 00 & .17 & .00 & .74 & .37 & .02 & .00 & .00 & .00 \\
\hline 20 & .00 & .00 & .00 & e. 00 & .11 & .00 & .29 & .09 & .01 & .00 & .00 & .00 \\
\hline 21 & .00 & e. 00 & .00 & e. 00 & .07 & .00 & .40 & .06 & .01 & .00 & .00 & .17 \\
\hline 22 & .00 & .00 & .00 & e. 00 & .06 & .00 & .49 & .03 & .01 & .00 & .00 & .16 \\
\hline 23 & .00 & .58 & .00 & e. 00 & .05 & .00 & .15 & .86 & .01 & .00 & .00 & .07 \\
\hline 24 & .00 & 2.7 & .00 & e. 00 & .03 & .00 & .37 & 1.3 & .03 & .00 & .00 & .01 \\
\hline 25 & .00 & .00 & .00 & e. 00 & .00 & .00 & .06 & .46 & .01 & .00 & .00 & .01 \\
\hline 26 & .00 & .00 & .00 & e. 00 & .00 & .00 & 1.1 & .28 & .01 & .00 & .00 & .00 \\
\hline 27 & .00 & .00 & .00 & e. 00 & .00 & .00 & .55 & 2.3 & .01 & .00 & .00 & .00 \\
\hline 28 & .00 & e. 00 & .00 & e. 00 & .00 & .00 & .19 & 1.5 & .15 & .00 & .00 & .00 \\
\hline 29 & .00 & e. 00 & .00 & e. 00 & $\cdots$ & .00 & .80 & 1.2 & .91 & .00 & .00 & .05 \\
\hline 30 & .00 & e. 00 & .00 & e. 00 & $\ldots$ & .00 & .63 & .66 & .12 & .00 & .00 & .16 \\
\hline 31 & .00 & $\cdots$ & .00 & e. 00 & $\cdots$ & .20 & $\cdots$ & .39 & $\cdots$ & .00 & .00 & $\cdots$ \\
\hline TOTAL & 0.10 & 3.50 & 0.00 & 0.00 & 1.85 & 0.95 & 7.05 & 13.66 & 8.16 & 0.09 & 0.00 & 0.63 \\
\hline MEAN & .003 & .12 & .000 & .000 & .066 & .031 & .23 & .44 & .27 & .003 & .000 & .021 \\
\hline $\operatorname{MAX}$ & .06 & 2.7 & .00 & .00 & .42 & .36 & 1.1 & 2.9 & 2.7 & $.0 b$ & .00 & .17 \\
\hline MIN & .00 & .00 & .00 & .00 & .00 & .00 & .00 & .00 & .01 & .00 & .00 & .00 \\
\hline$A C-F T$ & .2 & 6.9 & .00 & .00 & 3.7 & 1.9 & 14 & 27 & 16 & .2 & .00 & 1.2 \\
\hline \multicolumn{2}{|c|}{ STATISTICS OF } & MONTHLY MEAN & DATA & POR WATER & YEAR 1995 & & & & & & & \\
\hline MEAN & .003 & .12 & .000 & .000 & .066 & .031 & .23 & .44 & .27 & .003 & .000 & .021 \\
\hline MAX & .003 & .12 & .000 & .000 & .066 & .031 & .23 & .44 & .27 & .003 & .000 & .021 \\
\hline (WY) & 1995 & 1995 & 1995 & 1995 & 1995 & 1995 & 1995 & 1995 & 1995 & 1995 & 1995 & 1995 \\
\hline MIN & .003 & .12 & .000 & .000 & .066 & .031 & .23 & .44 & .27 & .003 & .000 & .021 \\
\hline (WY) & 1995 & 1995 & 1995 & $199 \mathrm{~b}$ & 1995 & 1995 & 1995 & 1995 & 1995 & 1995 & 1995 & 1995 \\
\hline
\end{tabular}

SUMMARY STATISTICS

FOR 199 b WATER YEAR

ANNUAL TOTAL

ANNUAL MEAN

HIGHEST DAILY MEAN

LOWEST DATLY MEAN

ANNUAL SEVEN-DAY MINIMUM

ANNUAL RUNOFF (AC-FT)

10 PERCENT EXCEEDS

50 PERCENT EXCEEDS

90 PERCENT EXCEEDS

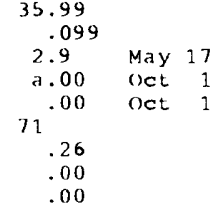

a No flow many days

e Estimated 
SITE NUMBER. - - SW029

STATION IDENTIFICATION. - 395310105113300

LOCATION. - Lat $39^{\circ} 53^{\prime} 10^{\prime \prime}$, long $105^{\circ} 11^{\prime} 33^{\prime \prime}$, in $\mathrm{SW}^{1} / 4 \mathrm{SE}^{1} / 4$ sec.11, T.2 S., R. $70 \mathrm{~W} .$, Jefferson County, Hydrologic Unit 10190003, Rocky Flats Environmental Technology site, outfall of Pond C-1 to Woman Creek.

DRAINAGE AREA. - - $1.26 \mathrm{mi}^{2}$.

PERIOD OF RECORD.--MaY 1994 to current year.

GAGE. - Water-stage recorder and v-notch weir, with broad-crested weir and culvert for high flows. Elevation of gage is $5,830 \mathrm{ft}$ above sea level.

REMARKS. - - Records good.

DISCHARGE, CUBIC FEET PER SECOND, WATER YEAR OCTOBER 2993 TO SEPTEMBER 1994

\begin{tabular}{|c|c|c|c|c|c|c|c|c|c|c|c|c|}
\hline DAY & $O C T$ & $\mathrm{~N}() \mathrm{V}$ & DEC & JAN & FEB & MAR & APR & MAY & JUN & JUL & $A \cup G$ & SEP \\
\hline 1 & $\cdots$ & $\cdots$ & $\cdots$ & $\cdots$ & $\cdots$ & $\cdots$ & $\cdots$ & $\cdots$ & .10 & .00 & .00 & .00 \\
\hline 2 & $\cdots$ & $\cdots$ & $\cdots$ & $\cdots$ & $\cdots$ & $\cdots$ & $\cdots$ & $\cdots$ & .06 & .00 & .00 & .00 \\
\hline 3 & $\cdots$ & $\cdots$ & $\cdots$ & $\cdots$ & $\cdots$ & $\cdots$ & $\cdots$ & $\cdots$ & .07 & .00 & .00 & .00 \\
\hline 4 & $\cdots$ & $\cdots$ & $\cdots$ & $\cdots$ & $\cdots$ & $\cdots$ & $\cdots$ & $\cdots$ & .11 & .00 & .00 & .00 \\
\hline 5 & $\cdots$ & $\cdots$ & $\cdots$ & $\cdots$ & $\cdots$ & $\cdots$ & $\cdots$ & $\cdots$ & .06 & .00 & .00 & .00 \\
\hline 6 & $\cdots$ & $\cdots$ & $\cdots$ & $\cdots$ & $\cdots$ & $\cdots$ & $\cdots$ & $\cdots$ & .03 & .00 & .00 & .00 \\
\hline 7 & $\cdots$ & $\cdots$ & $\cdots$ & $\cdots$ & $\cdots$ & $\cdots$ & $\cdots$ & $\cdots$ & .02 & .00 & .00 & .00 \\
\hline 8 & $\cdots$ & $\cdots$ & $\cdots$ & $\cdots$ & $\cdots$ & $\cdots$ & $\cdots$ & $\cdots$ & .01 & .00 & .00 & .0 \\
\hline 9 & $\cdots$ & $\cdots$ & $\cdots$ & $\cdots$ & $\cdots$ & $\cdots$ & $\cdots$ & $\cdots$ & .01 & .00 & .00 & .00 \\
\hline 10 & $\cdots$ & $\cdots$ & $\cdots$ & $\cdots$ & $\cdots$ & $\cdots$ & $\cdots$ & $\cdots$ & .01 & .00 & .00 & .00 \\
\hline 11 & $\cdots$ & $\cdots$ & $\cdots$ & $\cdots$ & $\cdots$ & $\cdots$ & $\cdots$ & .35 & .01 & .00 & .00 & .00 \\
\hline 12 & $\cdots$ & $\cdots$ & $\cdots$ & $\cdots$ & $\cdots$ & $\cdots$ & $\cdots$ & .49 & .02 & .00 & .00 & .00 \\
\hline 13 & $\cdots$ & $\cdots$ & $\cdots$ & $\cdots$ & $\cdots$ & $\cdots$ & $\cdots$ & .57 & .01 & .00 & .00 & .00 \\
\hline 14 & $\cdots$ & $\cdots$ & $\cdots$ & $\cdots$ & $\cdots$ & $\cdots$ & $\cdots$ & .75 & .00 & .00 & .00 & .00 \\
\hline 15 & $\cdots$ & $\cdots$ & $\cdots$ & $\cdots$ & $\cdots$ & $\cdots$ & $\cdots$ & .44 & .00 & .00 & .00 & .00 \\
\hline 16 & $\cdots$ & $\cdots$ & $\cdots$ & $\cdots$ & $\cdots$ & $\cdots$ & $\cdots$ & .32 & .00 & .00 & .00 & .00 \\
\hline 17 & $\cdots$ & $\cdots$ & $\cdots$ & $\cdots$ & $\cdots$ & $\cdots$ & $\cdots$ & .25 & .00 & .00 & .00 & .00 \\
\hline 18 & $\cdots$ & $\cdots$ & $\cdots$ & $\cdots$ & $\cdots$ & $\cdots$ & $\cdots$ & .23 & .00 & .00 & .00 & .00 \\
\hline 19 & $\cdots$ & $\cdots$ & $\cdots$ & $\cdots$ & $\cdots$ & $\cdots$ & $\cdots$ & .18 & .00 & .00 & .00 & .00 \\
\hline 20 & $\cdots$ & $\cdots$ & $\cdots$ & $\cdots$ & $\cdots$ & $\cdots$ & $\cdots$ & .15 & .00 & .00 & .00 & .00 \\
\hline 21 & $\cdots$ & $\cdots$ & $\cdots$ & $\cdots$ & $\cdots$ & $\cdots$ & $\cdots$ & .15 & .01 & .00 & .00 & .00 \\
\hline 22 & $\cdots$ & $\cdots$ & $\cdots$ & $\cdots$ & $\cdots$ & $\cdots$ & $\cdots$ & .18 & .03 & .00 & .00 & .00 \\
\hline 23 & $\cdots$ & $\cdots$ & $\cdots$ & $\cdots$ & $\cdots$ & $\cdots$ & $\cdots$ & .16 & .01 & .00 & .00 & .00 \\
\hline 24 & $\cdots$ & $\cdots$ & $\cdots$ & $\cdots$ & $\cdots$ & $\cdots$ & $\cdots$ & .13 & .00 & .00 & .00 & .00 \\
\hline 25 & $\cdots$ & $\cdots$ & $\cdots$ & $\cdots$ & $\cdots$ & $\cdots$ & $\cdots$ & .17 & .00 & .00 & .00 & .00 \\
\hline 26 & $\cdots$ & $\cdots$ & $\cdots$ & $\cdots$ & $\cdots$ & $\cdots$ & $\cdots$ & .18 & .00 & .00 & .00 & .00 \\
\hline 27 & $\cdots$ & $\cdots$ & $\cdots$ & $\cdots$ & $\cdots$ & $\cdots$ & $\cdots$ & .13 & .00 & .00 & .00 & .00 \\
\hline 28 & $\cdots$ & $\cdots$ & $\cdots$ & $\cdots$ & $\cdots$ & $\cdots$ & $\cdots$ & .13 & .00 & .00 & .00 & .00 \\
\hline 29 & $\cdots$ & $\cdots$ & $\cdots$ & $\cdots$ & $\cdots$ & $\cdots$ & $\cdots$ & .14 & .00 & .00 & .00 & .00 \\
\hline 30 & $\cdots$ & $\cdots$ & $\cdots$ & $\cdots$ & $\cdots$ & $\cdots$ & $\cdots$ & .07 & .00 & .00 & .00 & .00 \\
\hline 31 & $\cdots$ & $\cdots$ & $\cdots$ & $\cdots$ & $\cdots$ & $\cdots$ & $\cdots$ & .05 & $\cdots$ & .00 & .00 & $\cdots$ \\
\hline TOTAL & $\cdots$ & $\cdots$ & $\cdots$ & $\cdots$ & $\cdots$ & $\cdots$ & $\cdots$ & $\cdots$ & 0.57 & 0.00 & 0.00 & 0.00 \\
\hline MEAN & $\cdots$ & $\cdots$ & $\cdots$ & $\cdots$ & $\cdots$ & $\cdots$ & $\cdots$ & $\cdots$ & .029 & .000 & .000 & .000 \\
\hline MAX & $\cdots$ & $\cdots$ & $\cdots$ & $\cdots$ & $\cdots$ & $\cdots$ & $\cdots$ & $\cdots$ & .11 & . on & .00 & .00 \\
\hline MIN & $\cdots$ & $\cdots$ & $\cdots$ & $\cdots$ & $\cdots$ & $\cdots$ & $\cdots$ & $\cdots$ & .00 & .00 & .00 & .00 \\
\hline$A C \cdot F^{\prime} T$ & $\cdots$ & $\cdots$ & $\cdots$ & $\cdots$ & $\cdots$ & $\cdots$ & $\cdots$ & $\cdots$ & 1.1 & .00 & .00 & .00 \\
\hline
\end{tabular}


SITE NUMBER. - - SW029

STATION IDENTIFICATICN. - - 395310105113300

LOCATION. - - Lat $39^{\circ} 53^{\prime} 10^{\prime \prime}$, long $105^{\circ} 11^{\prime} 33^{\prime \prime}$, in $\mathrm{SW}^{1} / 4 \mathrm{SE}^{1} / 4 \mathrm{sec} .11, \mathrm{~T} .2 \mathrm{~S} ., \mathrm{R} .70$ w., Jefferson County, Hydrologic Unit 10190003 , Rocky Flats Environmental Technology Site, pond outfall to Woman Creek.

DRAINAGE AREA. $-1.26 \mathrm{mi}^{2}$.

PERIOD OF RECORD. - MaY 1994 to current year.

GAGE. - Water-stage recorder and V-notch weir, with broad-crested weir and culvert for high flows. Elevation of gage is $5,830 \mathrm{ft}$ above sea level.

REMARKS.--Records good for discharges less than $1.8 \mathrm{ft}^{3} / \mathrm{s}$. Records poor for discharges larger than $1.8 \mathrm{ft}^{3} / \mathrm{s}$, and for estimated daily discharges.

Discharge, CUbic FEET PER SECOND, WATER yeAR OCTUBER 1994 TO SEPTEMBER 1995

\begin{tabular}{|c|c|c|c|c|c|c|c|c|c|c|c|c|}
\hline DAY & OCT & NOV & DEC & JAN & FEB & MAR & APR & MAY & JUN & JUL & AUG & SEP \\
\hline 1 & .00 & .04 & .31 & .14 & .86 & .28 & .58 & 4.6 & 3.4 & .89 & 3.4 & .00 \\
\hline 2 & .00 & .04 & .33 & .10 & .66 & .23 & .33 & 4.0 & 3.5 & .50 & .21 & .00 \\
\hline 3 & .00 & .08 & .27 & .07 & .59 & .39 & .30 & 6.4 & 11 & .31 & .08 & .00 \\
\hline 4 & .00 & .14 & .27 & .04 & .47 & .47 & .24 & 5.7 & 6.5 & .25 & .05 & .00 \\
\hline 5 & .00 & .23 & .25 & .02 & .39 & .39 & .24 & 3.2 & 4.0 & .19 & .05 & .00 \\
\hline 6 & .00 & .24 & .25 & .05 & .35 & $.4 \mathrm{~s}$ & .19 & 3.1 & 1.1 & .10 & .03 & .00 \\
\hline 7 & .00 & .11 & .28 & .09 & .29 & .39 & .14 & 3.1 & .69 & .09 & .02 & .00 \\
\hline$B$ & .00 & .13 & .28 & .20 & .23 & .82 & .12 & 3.1 & 12 & .15 & .01 & .00 \\
\hline 9 & .00 & .24 & .17 & .35 & .22 & 1.9 & .17 & 2.0 & 39 & .15 & .00 & .00 \\
\hline 10 & .00 & .27 & .10 & .49 & .22 & .83 & .32 & .71 & 7.1 & .19 & .00 & .00 \\
\hline 11 & .00 & .14 & .10 & .41 & .18 & .33 & .54 & 1.4 & 4.6 & .14 & .00 & .00 \\
\hline 12 & .00 & .11 & .11 & .37 & .21 & .24 & 3.4 & .88 & 1.5 & .07 & .00 & .00 \\
\hline 13 & .00 & .11 & .11 & .30 & .16 & .14 & 1.6 & .72 & 1.1 & .06 & .00 & .00 \\
\hline 14 & .00 & .23 & .13 & .28 & .30 & .10 & .32 & 1.3 & .74 & .17 & .00 & .00 \\
\hline 15 & .00 & .27 & .10 & .35 & .87 & .13 & .06 & .95 & .59 & .20 & .00 & .00 \\
\hline 16 & .00 & .36 & .07 & .32 & .43 & .15 & .05 & .97 & .75 & .13 & .00 & .00 \\
\hline 17 & e. 50 & .57 & .12 & .18 & .65 & .23 & 1.2 & 93 & 1.2 & .11 & .00 & .00 \\
\hline 18 & el.0 & .57 & .18 & .08 & 1.6 & .18 & 2.5 & 1.4 & 5.8 & .10 & .00 & .00 \\
\hline 19 & .04 & .57 & .26 & .06 & 1.2 & .21 & 7.5 & 3.9 & 1.7 & .15 & .00 & .00 \\
\hline 20 & .03 & .51 & .28 & .13 & .91 & .29 & 6.9 & 2.3 & .86 & .22 & .00 & .00 \\
\hline 21 & .03 & .34 & .33 & .16 & .73 & .28 & 7.0 & 1.4 & .55 & .11 & .00 & .00 \\
\hline 22 & e. 03 & .22 & .36 & .09 & .50 & .19 & 9.7 & .93 & .39 & .06 & .00 & .00 \\
\hline 23 & e. 03 & .12 & .34 & .06 & .29 & .13 & 8.9 & 11 & .33 & .04 & .00 & .08 \\
\hline 24 & e. 03 & .18 & .36 & .06 & .26 & .07 & 11 & 20 & .81 & .04 & .00 & .09 \\
\hline 25 & .04 & .20 & .38 & .10 & .23 & .04 & 5.4 & B. 1 & .55 & .02 & .00 & .10 \\
\hline 26 & .04 & .19 & .39 & .19 & .20 & .08 & 15 & 6.0 & .31 & .01 & .00 & .09 \\
\hline 27 & .03 & .15 & .33 & .29 & .18 & .14 & 22 & 33 & .17 & .00 & .00 & .08 \\
\hline 28 & .02 & .09 & .30 & .24 & .27 & .19 & 7.5 & 19 & 1.3 & .00 & .00 & .07 \\
\hline 29 & .02 & .06 & .29 & .26 & $\cdots$ & .24 & 19 & 20 & 8.2 & 3.6 & .00 & .09 \\
\hline 30 & .03 & .11 & .29 & .22 & $\cdots$ & .35 & 17 & 12 & 1.7 & 7.8 & .00 & .19 \\
\hline 31 & .04 & & .23 & .44 & $\cdots$ & .72 & & 5.4 & & 6.6 & .00 & \\
\hline TOTAL, & 1.91 & 6.62 & 7.57 & 6.14 & 13.45 & 10.58 & 149.20 & 279.56 & 121.44 & 22.45 & 3.85 & 0.79 \\
\hline MEAN & .062 & .22 & .24 & .20 & .48 & .34 & 4.97 & 9.02 & 4.05 & .72 & .12 & .026 \\
\hline MAX & 1.0 & .57 & .39 & .49 & 1.6 & 1.9 & 22 & 93 & 39 & 7.8 & 3.4 & .19 \\
\hline MIN & .00 & .04 & .07 & .02 & .16 & .04 & .05 & .71 & .17 & .00 & .00 & .00 \\
\hline$A C \cdot F T$ & 3.8 & 13 & 15 & 12 & 27 & 21 & 296 & 555 & 241 & 45 & 7.6 & 1.6 \\
\hline
\end{tabular}

STATISTICS OF MUNTHLY MEAN DATA FOR WATRR YEARS 1994 - 1995, BY WATER YEAR (WY)

\begin{tabular}{|c|c|c|c|c|c|c|c|c|c|c|c|c|}
\hline MEAN & .062 & .22 & .24 & .20 & .48 & .34 & 4.91 & 9.02 & 2.03 & .36 & .062 & .013 \\
\hline MAX & .062 & .22 & .24 & .20 & .48 & .34 & 4.97 & 9.02 & 4.05 & .72 & .12 & .026 \\
\hline (WY) & 1995 & 1995 & 1995 & 1995 & 1995 & 1995 & 1995 & 1995 & 1995 & 1995 & 1995 & 1995 \\
\hline MIN & .062 & .22 & .24 & .20 & .48 & .34 & 4.97 & 9.02 & .019 & .000 & .000 & .000 \\
\hline (WY) & 1995 & 1995 & 1995 & 1995 & 1996 & 1995 & 1995 & 1995 & 1994 & 1994 & 1994 & 1994 \\
\hline
\end{tabular}

SUMMARY STATISTICS

FOR 199 S WA'TER YEAR

WATER YEARS 1994 - 1995

ANNUAL TOTAL

ANNUAL MEAN

HIGHEST ANNUAL MEAN

LOWEST ANNUAL MEAN

HIGHEST DAILY MEAN

LOWEST DAILY MEAN

ANNUAL SEVEN-DAY MINIMUM

ANNUAL RUNOFE (AC-FT)

10 PERCENT EXCEEDS

50 PERCENT EXCEEDS

90 PERCENT EXCEEOS

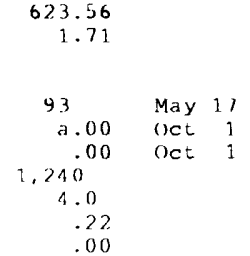

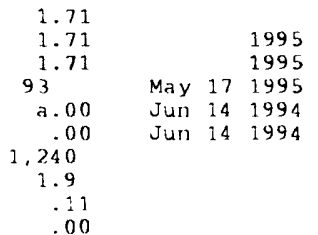

a No flow many days

e Estimated 
SITE NUMBER. - - SW093

STATION IDENTIFICATION. - - 395349105114900

LOCATION. - Lat $39^{\circ} 53^{\prime} 49^{\prime \prime}$, long $105^{\circ} 11^{\prime} 49^{\prime \prime}$, in $\mathrm{NE}^{1} / 4 \mathrm{NW}^{1} / 4 \mathrm{sec} .11, \mathrm{~T} .2$ s., R.70 W., Jefferson

County, Hydrologic Unit 10190003, Rocky Flats Environmental Technology Site, 1,000 feet above the A-1 Bypass, and 15 feet below a 60 -inch corrugated metal culvert.

DRAINAGE AREA. - - $0.36 \mathrm{mi}^{2}$.

PERIOD OF RECORD. - March 1994 to current year.

GAGE.- -Water-stage recorder and Parshall flume with weir plate. Elevation of gage is $5,895 \mathrm{ft}$ above sea level.

REMARKS. - - Records good.

DISCHARGE, CUBIC FEET PER SECOND, WATER YEAR OCTOBER 1993 TO SEPTEMBER 1994

\begin{tabular}{|c|c|c|c|c|c|c|c|c|c|c|c|c|}
\hline DAY & $O C T$ & NOV & DEC & JAN & $\mathrm{FEB}$ & MAR & APR & MAY & JUN & JUL & AUG & SEP \\
\hline 1 & $\cdots$ & $\cdots$ & $\cdots$ & $\ldots$ & $\cdots$ & $\cdots$ & .25 & .46 & .14 & .05 & .07 & .07 \\
\hline 2 & $\ldots$ & $\ldots$ & $\ldots$ & $\ldots$ & $\ldots$ & $\cdots$ & .25 & .44 & .09 & .05 & .07 & .03 \\
\hline 3 & $\ldots$ & $\ldots$ & $\ldots$ & $\cdots$ & $\cdots$ & $\cdots$ & .21 & .33 & .39 & .05 & .07 & .03 \\
\hline 4 & $\cdots$ & $\cdots$ & $\cdots$ & $\cdots$ & $\cdots$ & $\cdots$ & .20 & .26 & .14 & .05 & .07 & .03 \\
\hline 5 & $\cdots$ & $\cdots$ & $\cdots$ & $\cdots$ & $\cdots$ & $\cdots$ & .41 & .23 & .09 & .05 & .08 & .03 \\
\hline 6 & $\cdots$ & $\cdots$ & $\cdots$ & $\cdots$ & $\cdots$ & $\ldots$ & .31 & .20 & .08 & .05 & .07 & .02 \\
\hline 7 & $\cdots$ & $\cdots$ & $\cdots$ & $\cdots$ & $\cdots$ & $\cdots$ & .22 & .19 & .07 & .06 & .08 & .02 \\
\hline 8 & $\ldots$ & $\ldots$ & $\cdots$ & $\ldots$ & $\cdots$ & $\cdots$ & S & .17 & .07 & .06 & .08 & .02 \\
\hline 9 & $\ldots$ & $\ldots$ & $\ldots$ & $\ldots$ & $\cdots$ & $\cdots$ & .57 & $.2 b$ & .01 & .05 & .08 & .02 \\
\hline 10 & $\cdots$ & $\cdots$ & $\cdots$ & $\cdots$ & $\cdots$ & $\cdots$ & 2.8 & .18 & .07 & .05 & .51 & .02 \\
\hline 11 & $\cdots$ & $\ldots$ & $\ldots$ & $\cdots$ & $\cdots$ & $\cdots$ & .87 & .18 & .06 & .05 & .45 & .02 \\
\hline 12 & $\ldots$ & $\cdots$ & $\cdots$ & $\cdots$ & $\cdots$ & $\cdots$ & 1.1 & .17 & .06 & .06 & .06 & .03 \\
\hline 13 & $\cdots$ & $\cdots$ & $\cdots$ & $\cdots$ & $\cdots$ & $\cdots$ & .50 & .72 & .05 & .05 & .06 & .03 \\
\hline 14 & $\ldots$ & $\cdots$ & $\cdots$ & $\cdots$ & $\cdots$ & $\ldots$ & .36 & .28 & .05 & .06 & .05 & .03 \\
\hline 15 & $\cdots$ & $\cdots$ & $\cdots$ & $\cdots$ & $\cdots$ & $\cdots$ & .30 & .18 & .06 & .05 & .03 & .03 \\
\hline 16 & $\ldots$ & $\cdots$ & $\ldots$ & $\cdots$ & $\cdots$ & $\cdots$ & .26 & .19 & .05 & .05 & .01 & .03 \\
\hline 17 & $\cdots$ & $\cdots$ & $\cdots$ & $\cdots$ & $\cdots$ & $\ldots$ & .23 & .14 & .06 & .06 & .02 & .03 \\
\hline 18 & $\cdots$ & $\cdots$ & $\cdots$ & $\cdots$ & $\cdots$ & $\cdots$ & .21 & .11 & .07 & .05 & .02 & .03 \\
\hline 19 & $\ldots$ & $\cdots$ & $\cdots$ & $\ldots$ & $\cdots$ & $\ldots$ & .19 & .10 & .08 & .05 & .05 & .03 \\
\hline 20 & $\cdots$ & $\cdots$ & $\cdots$ & $\cdots$ & $\cdots$ & $\cdots$ & .18 & .10 & .08 & .05 & .03 & .04 \\
\hline 21 & $\cdots$ & $\cdots$ & $\cdots$ & $\cdots$ & $\cdots$ & .08 & .17 & .08 & .28 & .05 & .03 & .24 \\
\hline 22 & $\cdots$ & $\cdots$ & $\cdots$ & $\cdots$ & $\cdots$ & .08 & .17 & .10 & .17 & .05 & .02 & .11 \\
\hline 23 & $\cdots$ & $\cdots$ & $\cdots$ & $\cdots$ & $\cdots$ & .07 & .16 & .11 & .07 & .05 & .02 & .04 \\
\hline 24 & $\cdots$ & $\cdots$ & $\cdots$ & $\cdots$ & $\cdots$ & .07 & .14 & .08 & .06 & .06 & .02 & .03 \\
\hline 25 & $\cdots$ & $\cdots$ & $\cdots$ & $\cdots$ & $\cdots$ & .08 & 1.3 & .11 & .05 & .05 & .03 & .03 \\
\hline 26 & $\cdots$ & $\cdots$ & $\cdots$ & $\cdots$ & $\cdots$ & .10 & .40 & .10 & .05 & .05 & .03 & .03 \\
\hline 27 & $\cdots$ & $\cdots$ & $\cdots$ & $\cdots$ & $\cdots$ & .30 & .48 & .09 & .05 & .07 & .03 & .03 \\
\hline 28 & $\cdots$ & $\ldots$ & $\cdots$ & $\cdots$ & $\cdots$ & .23 & .41 & .31 & .05 & .06 & .06 & .03 \\
\hline 29 & $\cdots$ & $\cdots$ & $\cdots$ & $\cdots$ & $\cdots$ & .89 & 1.6 & .10 & .05 & .06 & .06 & .03 \\
\hline 30 & $\cdots$ & $\cdots$ & $\cdots$ & $\cdots$ & $\cdots$ & .57 & .86 & .08 & .05 & .06 & .03 & .03 \\
\hline 31 & $\cdots$ & $\cdots$ & $\cdots$ & $\cdots$ & $\cdots$ & .32 & $\cdots$ & .33 & $\cdots$ & .06 & .08 & $\cdots$ \\
\hline TOTAL & $\cdots$ & $\cdots$ & $\cdots$ & $\cdots$ & $\cdots$ & $\cdots$ & 14.26 & 6.37 & 2.71 & 1.67 & 2.37 & 1.19 \\
\hline MEAN & $\cdots$ & $\cdots$ & $\cdots$ & $\cdots$ & $\cdots$ & $\cdots$ & .48 & .21 & .090 & .054 & .076 & .040 \\
\hline $\operatorname{MAX}$ & $\cdots$ & $\cdots$ & $\cdots$ & $\cdots$ & $\cdots$ & $\cdots$ & 1.8 & .72 & .39 & .07 & .51 & .24 \\
\hline MIN & $\cdots$ & $\cdots$ & $\cdots$ & $\cdots$ & $\cdots$ & $\cdots$ & .14 & .08 & .05 & .05 & .01 & .02 \\
\hline$A C-F T$ & $\cdots$ & $\cdots$ & $\cdots$ & $\cdots$ & $\cdots$ & $\cdots$ & 28 & 13 & 5.4 & 3.3 & 4.7 & 2. \\
\hline
\end{tabular}


WALNUT CREEK BELOW PORTAL 3

SITE NUMBER. - SW093

STATION IDENTIFICATION. - 395349105114900

LOCATION. - Lat $39^{\circ} 53^{\prime} 49^{\prime \prime}$, Iong $105^{\circ} 11^{\prime} 49^{\prime \prime}$, in $\mathrm{NE}^{1} / 4 \mathrm{NW}^{1} / 4$ sec.11, T.2 S., R.70 W. , Jefferson

County, Hydrologic Unit 10190003, Rociky Flats Environmental Technology site, 1,000 feet above the A-1 Bypass, and 15 feet below a 60-inch corrugated metal culvert.

DRAINAGE AREA. - $0.36 \mathrm{mi}^{2}$.

PERIOD OF RECORD. - March 1994 to current year.

GAGE. - Water-stage recorder and parshall flume with weir plate. Elevation of gage is 5,895 ft above sea level.

REMARKS.--Records good except for estimated daily discharges, which are poor.

DISCHARGE, CUBIC FEET PER SECOND, WATER YEAR OCTOBER 1994 TO SEPTEMBER 1995 DA ILY MEAN VALUES

\begin{tabular}{|c|c|c|c|c|c|c|c|c|c|c|c|c|}
\hline DAY & OCT & NOV & DEC & JAN & - $\mathrm{FEB}$ & MAR & A PR & MAY & $J U N$ & JUL & AUG & SEP \\
\hline 1 & .08 & .07 & .08 & e. 00 & .12 & .03 & .12 & .71 & .63 & .19 & .05 & .06 \\
\hline 2 & .14 & .06 & .07 & e.00 & .07 & .00 & .11 & .71 & e1. 4 & .13 & .04 & .06 \\
\hline 3 & .05 & .11 & .04 & e. 00 & .08 & .00 & .09 & .97 & e1.8 & .10 & .04 & .05 \\
\hline 4 & .04 & .36 & e. 04 & e.00 & .06 & .00 & .08 & .60 & 1.4 & .09 & .05 & .06 \\
\hline 5 & .04 & .23 & e. 04 & e. 00 & .06 & .00 & .08 & .43 & .60 & .11 & .04 & .06 \\
\hline 6 & .05 & .12 & e. 08 & e. 00 & .05 & .00 & .07 & .40 & .48 & .05 & .03 & .07 \\
\hline 7 & .05 & .10 & e.07 & e. 00 & .05 & .12 & .07 & .36 & .37 & .03 & .03 & .08 \\
\hline 8 & .04 & .72 & e. 09 & e.00 & .05 & .19 & .07 & .33 & 2.9 & .03 & .03 & .08 \\
\hline 9 & .04 & .38 & e. 08 & e.00 & .06 & e. 15 & .12 & .30 & 4.7 & .02 & .03 & .16 \\
\hline 10 & .04 & .09 & e. 08 & e. 00 & e. 05 & .10 & .50 & .29 & .75 & .02 & .04 & .08 \\
\hline 11 & .03 & .04 & e. 06 & e.00 & e. 01 & .08 & .63 & .29 & .55 & .02 & .04 & .08 \\
\hline 12 & .04 & .03 & e. 06 & e. 00 & e. 00 & .08 & .75 & .27 & .41 & .01 & .05 & .08 \\
\hline 13 & .05 & .02 & e. 06 & e. 03 & e. 00 & .07 & .33 & .26 & .35 & .00 & .04 & .07 \\
\hline 14 & .03 & .32 & e. 06 & e. 04 & e. 60 & .06 & .19 & .23 & .32 & .09 & .05 & .08 \\
\hline 15 & .06 & .08 & e. 04 & e. 04 & .19 & .06 & .12 & .19 & .29 & .06 & .04 & .08 \\
\hline 16 & .06 & .09 & e. 07 & e.03 & .09 & .08 & .39 & 1.0 & .23 & .04 & .04 & .08 \\
\hline 17 & .53 & .08 & e. 07 & e. 04 & .23 & .08 & 1.5 & el1 & .54 & .03 & .05 & .08 \\
\hline 18 & .06 & .05 & e. 07 & e.03 & .17 & .06 & 1.7 & i. 6 & 30 & .24 & .28 & .30 \\
\hline 19 & .05 & .05 & e. 09 & e. 03 & .12 & .06 & 1.9 & .75 & .20 & .12 & .14 & .28 \\
\hline 20 & .05 & .07 & e. 09 & e. 03 & .10 & .05 & .85 & .55 & .18 & .06 & .09 & .51 \\
\hline 21 & .05 & .08 & .03 & e. 04 & .11 & .06 & 2.2 & .44 & .17 & .05 & .05 & .76 \\
\hline 22 & .05 & .08 & e. 01 & e. 04 & .15 & .06 & 1.3 & .43 & .16 & .04 & .05 & .30 \\
\hline 23 & .05 & .09 & e. 00 & e.04 & .14 & .05 & 1.1 & 3.3 & .27 & .04 & .05 & .14 \\
\hline 24 & .05 & .08 & e. 00 & e. 04 & .10 & .05 & .99 & 2.9 & .25 & .04 & .05 & .15 \\
\hline 25 & .05 & .08 & e. 00 & e.0b & .05 & .05 & 1.6 & 1.3 & .17 & .04 & .05 & .08 \\
\hline 26 & .05 & .08 & e. 00 & .05 & .05 & .18 & 3.3 & e2.1 & .13 & .04 & .05 & .06 \\
\hline 27 & .06 & .08 & e. 00 & .05 & .05 & .09 & 1.7 & e4.1 & .09 & .03 & .05 & .05 \\
\hline 28 & .08 & .08 & e. 00 & .05 & .05 & .28 & 3.4 & e4.3 & .74 & .03 & .05 & .05 \\
\hline 29 & .07 & .06 & e. 00 & .10 & $\cdots$ & .21 & 2.3 & 2.0 & 2.3 & .04 & .05 & .21 \\
\hline 30 & .06 & .07 & e. 00 & .08 & $\ldots$ & .20 & 2.3 & 1.5 & .29 & .04 & .05 & .43 \\
\hline 31 & .07 & $\cdots$ & e. 00 & .12 & $\cdots$ & .17 & $\cdots$ & 1.3 & $\cdots$ & .05 & .05 & $\cdots$ \\
\hline TOTAL & 2.17 & 3.85 & 1.38 & 0.93 & 2.86 & 2.67 & 27.86 & 44.91 & 22.97 & 1.88 & 1.75 & 4.63 \\
\hline MEAN & .070 & .13 & .045 & .030 & .10 & .086 & .93 & 1.45 & .77 & .061 & .056 & .15 \\
\hline $\operatorname{MAX}$ & .53 & .72 & .09 & .12 & .60 & .28 & 3.3 & 11 & 4.7 & .24 & .28 & .76 \\
\hline MIN & .03 & .02 & .90 & .00 & .00 & .00 & .07 & .19 & .09 & .00 & .03 & .05 \\
\hline$A C-F T$ & 4.3 & 7.6 & 2.7 & 1.8 & 5.1 & 5.3 & 55 & 89 & 46 & 3.7 & 3.5 & 9.2 \\
\hline
\end{tabular}

STATISTICS OF MONTHLY MEAN DATA FOR WATER YEARS 1994 - 1995, BY WATER YEAR (WY)

$\begin{array}{lrrrr}\text { MEAN } & .070 & .13 & .045 & .030 \\ \text { MAX } & .070 & .13 & .045 & .030 \\ \text { (WY) } & 1995 & 1995 & 1995 & 1995 \\ \text { MIN } & .070 & .13 & .045 & .030 \\ \text { (WY) } & 1995 & 1995 & 1995 & 1995\end{array}$

SUMMARY STATISTICS

ANNUAL, TOTAL,

ANNUAL, MEAN

HIGHEST ANNUAL MEAN

LOWEST ANNUAL MEAN

HIGHEST DAILY MEAN

LOWEST DAILY MEAN

ANNUAL SEVEN-DAY MINIMUM

ANNUAL RUNOFF (AC-FT)

10 PERCENT EXCEEDS

50 PERCENT EXCEEDS

0 PERCENT EXCEEDS

$\begin{array}{rr}.10 & .086 \\ .10 & .086 \\ 1995 & 1995 \\ .10 & .086 \\ 1995 & 1995\end{array}$

.70
.93
1995
.48
1994

.83
$\square .45$
$\square 995$
.21
$\square 994$

FOR 1995 WATER YEAR

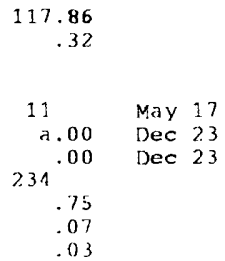

.43
.77
1995
.090
1994

.05
.061
1995
.054
1994

WATER YEARS 1994 - 1995

a No flow many days

b No flow at times most years

e Estimated 
GRAVEL PIT AT ROCKY FLATS

SITE NUMBER. - - SW134

STATION IDENIIFICATION. - 395331105134400

LOCATION. - Lat $39^{\circ} 53^{\prime} 31^{\prime \prime}$, long $105^{\circ} 13^{\prime} 44^{\prime \prime}$, in $\mathrm{NE}^{1} / 4 \mathrm{SW}^{1} / 4$ sec.9, T. 2 S., R. 70 W., Jefierson County, Hydrologic Unit 10190003, Rocky Flats Environmental Technology site, at discharge point for surface water ponded in gravel pits situated in upper Rock Creek Basin.

DRAINAGE AREA.--Unknown; pumped direct discharge from gravel pits.

PERIOD OF RECORD. - MaY 1994 to current year.

GAGE.- Water-stage recorder and Parshall flume. Elevation of gage is $6,150 \mathrm{ft}$ above sea level.

REMARKS.--Records fair except for estimated daily discharges, which are poor. Discharge, CUBIC FEET PER SECOND, WATER YEAR ICTOBER 1993 TO SEPTEMBER 1994

\begin{tabular}{|c|c|c|c|c|c|c|c|c|c|c|c|c|}
\hline DAY & XCT & NOV & DEC & JAN & FEB & MAR & APR & MAY & JUN & JUL & AUG & SEP \\
\hline 1 & $\cdots$ & $\cdots$ & .. & $\cdots$ & $\cdots$ & $\cdots$ & $\ldots$ & $\ldots$ & .00 & .00 & .00 & .00 \\
\hline 2 & $\cdots$ & $\cdots$ & $\cdots$ & $\cdots$ & $\cdots$ & $\cdots$ & $\ldots$ & $\cdots$ & .03 & .00 & .00 & .00 \\
\hline 3 & $\ldots$ & $\ldots$ & $\ldots$ & $\cdots$ & $\ldots$ & $\cdots$ & $\cdots$ & $\ldots$ & .00 & .00 & .00 & .00 \\
\hline 4 & $\cdots$ & $\cdots$ & $\cdots$ & $\cdots$ & $\cdots$ & $\cdots$ & $\cdots$ & .00 & .00 & .00 & .00 & .00 \\
\hline 5 & $\cdots$ & $\cdots$ & $\cdots$ & $\cdots$ & $\cdots$ & $\cdots$ & $\cdots$ & .00 & .00 & .00 & .00 & .00 \\
\hline 6 & $\cdots$ & $\cdots$ & $\cdots$ & $\cdots$ & $\cdots$ & $\cdots$ & $\cdots$ & .00 & .00 & .00 & .03 & .00 \\
\hline 7 & $\cdots$ & $\cdots$ & $\cdots$ & $\cdots$ & $\cdots$ & $\cdots$ & $\cdots$ & .00 & .00 & .00 & .00 & .00 \\
\hline 8 & $\cdots$ & $\cdots$ & $\cdots$ & $\cdots$ & $\cdots$ & $\ldots$ & $\ldots$ & .00 & .00 & .00 & .00 & .00 \\
\hline 9 & $\ldots$ & $\ldots$ & $\cdots$ & $\ldots$ & $\ldots$ & $\ldots$ & $\cdots$ & .07 & .00 & .00 & .00 & .00 \\
\hline 10 & $\cdots$ & $\cdots$ & $\cdots$ & $\cdots$ & $\cdots$ & $\cdots$ & $\cdots$ & .00 & .03 & .00 & .00 & .07 \\
\hline 11 & $\cdots$ & $\cdots$ & $\cdots$ & $\cdots$ & $\cdots$ & $\cdots$ & $\cdots$ & .00 & .00 & .00 & .02 & .00 \\
\hline 12 & $\cdots$ & $\cdots$ & $\cdots$ & $\cdots$ & $\cdots$ & $\cdots$ & $\ldots$ & .00 & .00 & .00 & .00 & .00 \\
\hline 13 & $\cdots$ & $\cdots$ & $\cdots$ & $\cdots$ & $\cdots$ & $\cdots$ & $\cdots$ & .00 & .00 & .00 & .00 & .00 \\
\hline 14 & $\cdots$ & $\cdots$ & $\cdots$ & $\cdots$ & $\cdots$ & $\cdots$ & $\cdots$ & .00 & .00 & .00 & .00 & .00 \\
\hline 15 & $\cdots$ & $\cdots$ & $\cdots$ & $\cdots$ & $\cdots$ & $\cdots$ & $\cdots$ & .00 & .00 & .00 & .00 & .00 \\
\hline 16 & $\ldots$ & $\cdots$ & $\ldots$ & $\cdots$ & $\ldots$ & $\ldots$ & $\cdots$ & .00 & e. 00 & .00 & .00 & .00 \\
\hline 17 & $\cdots$ & $\cdots$ & $\ldots$ & $\ldots$ & $\cdots$ & $\cdots$ & $\cdots$ & .00 & .00 & .00 & .00 & .00 \\
\hline 18 & $\ldots$ & $\cdots$ & $\cdots$ & $\cdots$ & $\ldots$ & $\cdots$ & $\ldots$ & e. 00 & .00 & .00 & .00 & .00 \\
\hline 19 & $\cdots$ & $\cdots$ & $\cdots$ & $\cdots$ & $\cdots$ & $\cdots$ & $\cdots$ & .00 & .04 & .00 & .00 & .00 \\
\hline 20 & $\cdots$ & $\cdots$ & $\cdots$ & $\cdots$ & $\cdots$ & $\cdots$ & $\ldots$ & .00 & .00 & .00 & .00 & .00 \\
\hline 21 & $\cdots$ & $\cdots$ & $\cdots$ & $\ldots$ & $\cdots$ & $\cdots$ & $\cdots$ & .00 & .00 & .00 & .00 & .00 \\
\hline 22 & $\cdots$ & $\ldots$ & $\cdots$ & $\cdots$ & $\cdots$ & $\cdots$ & $\cdots$ & .00 & .00 & .06 & .00 & .00 \\
\hline 23 & $\ldots$ & $\cdots$ & $\cdots$ & $\cdots$ & $\ldots$ & $\cdots$ & $\ldots$ & .00 & .00 & .00 & .00 & .00 \\
\hline 24 & $\cdots$ & $\ldots$ & - . & $\ldots$ & $\ldots$ & $\cdots$ & $\cdots$ & .00 & .00 & .00 & .00 & .00 \\
\hline 25 & $\cdots$ & $\ldots$ & $\cdots$ & $\cdots$ & $\cdots$ & $\cdots$ & $\cdots$ & .00 & .00 & .00 & .00 & .00 \\
\hline 26 & $\cdots$ & $\cdots$ & $\cdots$ & $\ldots$ & $\cdots$ & $\ldots$ & $\cdots$ & .00 & .00 & .00 & .00 & .00 \\
\hline 27 & $\ldots$ & $\ldots$ & $\ldots$ & $\ldots$ & $\ldots$ & $\ldots$ & $\ldots$ & .00 & .04 & .00 & .00 & .00 \\
\hline 28 & $\cdots$ & $\ldots$ & $\cdots$ & $\cdots$ & $\cdots$ & $\ldots$ & $\cdots$ & .00 & .00 & .00 & .00 & .00 \\
\hline 29 & $\cdots$ & $\cdots$ & $\cdots$ & $\cdots$ & $\ldots$ & $\cdots$ & $\cdots$ & .00 & .00 & .00 & .00 & .00 \\
\hline 30 & $\cdots$ & $\cdots$ & $\ldots$ & $\ldots$ & $\ldots$ & $\cdots$ & $\cdots$ & .00 & .00 & .00 & .00 & .00 \\
\hline 31 & $\cdots$ & $\cdots$ & $\cdots$ & $\cdots$ & $\cdots$ & $\cdots$ & $\cdots$ & .00 & $\cdots$ & .00 & .00 & $\ldots$ \\
\hline TOI'AL & $\cdots$ & $\cdots$ & $\cdots$ & $\cdots$ & $\ldots$ & $\cdots$ & $\cdots$ & $\ldots$ & 0.14 & 0.06 & 0.05 & 0.07 \\
\hline MEAN & $\cdots$ & $\ldots$ & $\ldots$ & $\cdots$ & $\ldots$ & $\cdots$ & $\ldots$ & $\cdots$ & .005 & .002 & .002 & .002 \\
\hline $\operatorname{MAX}$ & $\cdots$ & $\ldots$ & $\cdots$ & $\cdots$ & $\cdots$ & $\cdots$ & $\cdots$ & $\cdots$ & .04 & .06 & .03 & .07 \\
\hline MIN & $\ldots$ & $\cdots$ & $\cdots$ & $\cdots$ & $\cdots$ & $\cdots$ & $\cdots$ & $\cdots$ & .00 & .00 & .00 & .00 \\
\hline$A C-F T$ & $\ldots$ & $\cdots$ & $\ldots$ & $\ldots$ & $\ldots$ & $\ldots$ & $\ldots$ & $\ldots$ & .3 & .1 & .1 & .1 \\
\hline
\end{tabular}

e Estimated 
SITE NUMBER. - - SW134

STATION IDENTIFICATION. - - 395331105134400

LOCATION. - Lat $39^{\circ} 53^{\prime} 31^{\prime \prime}$, long $105^{\circ} 13^{\prime} 44^{\prime \prime}$, in $\mathrm{NE}^{1} / 4 \mathrm{SW}^{1} / 4$ sec.9, T.2 S., R. 70 W., Jefferson County, Hydrologic Unit 10190003 , Rocky Flats Environmental Technology site, at discharge point for surface water ponded in gravel pits situated in upper Rock Creek Basin.

DRAINAGE AREA.--Unknown; pumped direct discharqe from qravel pits.

PERIOD OF RECORD. - -MaY 1994 to current year.

GAGE. - Water-stage recorder and Parshall flume. Elevation of gage is $6.150 \mathrm{ft}$ above sea level.

REMARKS. - Records fair except for estimated daily discharges, which are poor.

DISCHARGE, CUBIC FEET PER SECOND, WATER YEAR OCTOBER 1994 TO SEPTEMBER 1995

\begin{tabular}{|c|c|c|c|c|c|c|c|c|c|c|c|c|}
\hline DAY & $O C T$ & NOV & $D E C$ & JAN & FEB & $M A R$ & $A P R$ & MAY & JUN & JUL, & $A \cup G$ & SEP \\
\hline 1 & .00 & .00 & .00 & e. 00 & .00 & e. 00 & .00 & .13 & .10 & .00 & .00 & .00 \\
\hline 2 & .00 & .00 & .00 & e. 00 & .00 & e. 00 & .00 & .00 & .28 & .00 & .00 & .00 \\
\hline 3 & .00 & .00 & .00 & e. 00 & .00 & .00 & .00 & .00 & .00 & .00 & .00 & .00 \\
\hline 4 & .00 & .00 & .00 & e. 00 & .00 & .00 & .00 & .05 & .00 & .00 & .00 & .00 \\
\hline 5 & .00 & .00 & .00 & e. 00 & .00 & .00 & .00 & .26 & .11 & .00 & .06 & .04 \\
\hline 6 & .00 & .00 & .00 & .00 & .00 & .00 & .00 & .00 & .36 & .05 & .00 & .00 \\
\hline 7 & .00 & .00 & .00 & .00 & .00 & .23 & .00 & .00 & .00 & .00 & .00 & .00 \\
\hline 8 & .04 & .00 & e. 00 & .00 & .00 & e. 00 & .00 & .00 & .01 & .00 & .00 & .02 \\
\hline 9 & .00 & .00 & e.00 & .00 & .00 & .00 & .00 & .21 & .49 & .00 & .00 & .00 \\
\hline 10 & .00 & .00 & e. 00 & .00 & e. 00 & .00 & .00 & .00 & .00 & .00 & .00 & .00 \\
\hline 11 & .00 & .00 & e. 00 & .00 & .00 & .00 & .00 & .05 & .00 & .00 & .00 & .00 \\
\hline 12 & .00 & .00 & e. 00 & .00 & .00 & .00 & .14 & .00 & .12 & .00 & .00 & .00 \\
\hline 13 & .00 & .00 & e. 00 & .00 & .00 & .00 & .00 & .00 & .00 & .00 & .00 & .00 \\
\hline 14 & .00 & .00 & e. 00 & .00 & e. 00 & .00 & .00 & .00 & .00 & .02 & .00 & .00 \\
\hline 15 & .00 & .06 & e. 00 & .00 & e. 00 & .00 & .00 & .00 & .04 & .00 & .00 & .05 \\
\hline 16 & .00 & .08 & e. 00 & .00 & .00 & .00 & .00 & .00 & .00 & .00 & .00 & .00 \\
\hline 17 & .00 & e. 00 & .00 & .00 & .00 & .00 & .00 & .79 & .00 & .03 & .00 & .00 \\
\hline 18 & .00 & e. 00 & .00 & e. 00 & .00 & .00 & .00 & .43 & .00 & .00 & .10 & .00 \\
\hline 19 & .00 & e. 00 & .00 & .00 & .00 & .00 & .00 & .48 & .00 & .00 & .00 & .00 \\
\hline 20 & .00 & e. 00 & .00 & .13 & .00 & .00 & .02 & .26 & .05 & .00 & .00 & .00 \\
\hline 21 & .00 & e. 00 & .00 & e. 00 & .00 & .00 & .08 & .00 & .00 & .00 & .00 & .00 \\
\hline 22 & .00 & e. 00 & .00 & e. 00 & .16 & .00 & .14 & .28 & .00 & .00 & .00 & .00 \\
\hline 23 & .00 & e. 00 & .00 & e. 00 & .00 & .07 & .00 & .10 & .05 & .00 & .06 & .00 \\
\hline 24 & .00 & .00 & .00 & e. 00 & .00 & .00 & .08 & .20 & .07 & .00 & .00 & .00 \\
\hline 25 & .03 & .00 & .00 & e. 00 & .00 & .00 & .47 & .04 & .00 & .00 & .01 & .00 \\
\hline 26 & .00 & .00 & .00 & .00 & .00 & .00 & .38 & .31 & .08 & .00 & .00 & .18 \\
\hline 27 & .00 & e.00 & .00 & .00 & .00 & .00 & .11 & .01 & .01 & .00 & .00 & .05 \\
\hline 28 & .00 & e. 00 & .00 & .00 & e. 00 & .00 & .42 & .01 & .00 & .09 & .00 & .00 \\
\hline 29 & .00 & e. 00 & .00 & .00 & $\ldots$ & e. 00 & .00 & .17 & .06 & .00 & .00 & .00 \\
\hline 30 & .00 & .00 & .00 & .00 & $\cdots$ & e. 00 & .00 & .41 & .13 & .00 & .04 & .00 \\
\hline 31 & .00 & $\cdots$ & .00 & .00 & $\cdots$ & e. 00 & $\cdots$ & .10 & $\ldots$ & .00 & .00 & $\cdots$ \\
\hline TOTAL & 0.07 & 0.14 & 0.00 & 0.13 & 0.16 & 0.30 & 1.84 & 4.29 & 1.96 & 0.19 & 0.27 & 0.34 \\
\hline MEAN & .002 & .005 & .000 & .004 & .006 & .010 & .061 & .14 & .065 & .006 & .009 & .011 \\
\hline $\operatorname{MAX}$ & .04 & .08 & .00 & .13 & .16 & .23 & .47 & .79 & .49 & .09 & .10 & .18 \\
\hline MIN & .00 & .00 & .00 & .00 & .00 & .00 & .00 & .00 & .00 & .00 & .00 & .00 \\
\hline$A C-E T$ & .1 & .3 & .00 & .3 & .3 & .6 & 3.6 & 8.5 & 3.9 & .4 & .5 & .7 \\
\hline
\end{tabular}

STATISTICS OF MONTHLY MEAN DATA FOR WATER YEARS 1994 - 1995, BY WATER YEAR (WY)

\begin{tabular}{|c|c|c|c|c|c|c|c|c|c|c|c|c|}
\hline MEAN & .002 & .005 & .000 & .004 & .006 & .010 & .061 & .14 & .035 & .004 & .005 & .007 \\
\hline $\operatorname{MAX}$ & .002 & .005 & .000 & .004 & .006 & .010 & .061 & .14 & .065 & .006 & .009 & .011 \\
\hline (WY) & 1995 & 1995 & 1995 & 1995 & 1995 & 1995 & 1995 & 1995 & 1995 & 1995 & 1995 & 1995 \\
\hline MIN & .002 & .005 & .000 & .004 & .006 & .010 & .061 & .14 & .005 & .002 & .002 & .002 \\
\hline (WY) & 1995 & 1995 & 1995 & 1995 & 1995 & 1995 & 1995 & 1995 & 1994 & 1994 & 1994 & 199 \\
\hline
\end{tabular}

SUMMARY STATISTICS

FOR 1995 WATER YEAR

WATER YEARS $1994 \cdot 1995$

ANNUAL TOTAL

ANNUAL MEAN

HIGHEST ANNUAL MEAN

LOWEST ANNUAL MEAN

HIGHEST DAILY MEAN

ANNUAL SEVEN-DAY MINIMUM

ANNUAL RUNOFF (AC-ET)

10 PERCENT EXCEEDS

SO PERCENT EXCEEDS

90 PERCENT EXCEEDS

a No flow many days

e Estimater?
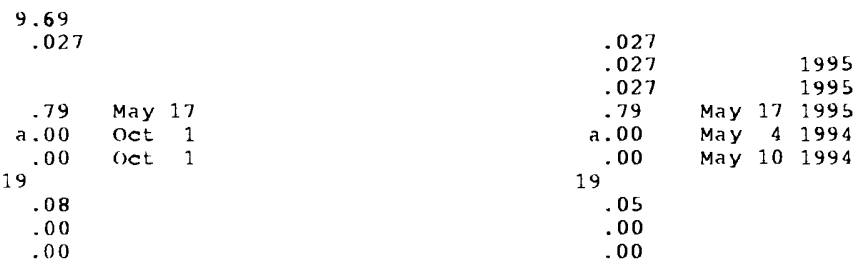
SITE NUMBER. - - SW998

STATION IDENTIFICATION. - 395332105124600

LOCATION. - Lat $39^{\circ} 53^{\prime} 32^{\prime \prime}$, long $105^{\circ} 12^{\prime} 46^{\prime \prime}$, in $\mathrm{SW}^{1} / 4 \mathrm{SW}^{1} / 4$ sec. 10 , T.2 S., R.70 W., Jefferson County, Hydrologic Unit 10190003, Rocky Flats Environmental Technology Site, on West Diversion Ditch downstream from the 130 -building complex.

DRAINAGE AREA. - $0.33 \mathrm{mi}^{2}$.

PERIOD OF RECORD. - MaY 1994 to current year.

GAGE. - Water-stage recorder and Parshall flume with weir plate. Elevation of gage is 6,047 ft above sea level.

REMARKS. - Records poor.

DISCHARGE, CUBIC FEET PER SECOND, WATER YEAR OCTOBER 1993 TO SEPTEMBER 1994

\begin{tabular}{|c|c|c|c|c|c|c|c|c|c|c|c|c|}
\hline DAY & WT & Nov & DEC & JAN & FEB & MAR & APR & MAY & JUN & JUL & AUG & SEP \\
\hline 1 & $\cdots$ & $\cdots$ & $\cdots$ & $\cdots$ & $\cdots$ & $\cdots$ & $\cdots$ & $\cdots$ & .01 & .00 & .00 & .03 \\
\hline 2 & $\cdots$ & $\cdots$ & $\cdots$ & $\cdots$ & $\cdots$ & $\cdots$ & $\cdots$ & $\cdots$ & .00 & .00 & .00 & .00 \\
\hline 3 & $\cdots$ & $\cdots$ & $\cdots$ & $\cdots$ & $\cdots$ & $\cdots$ & $\cdots$ & $\cdots$ & .20 & .00 & .00 & .00 \\
\hline 4 & $\cdots$ & $\cdots$ & $\cdots$ & $\cdots$ & $\cdots$ & $\cdots$ & $\cdots$ & $\cdots$ & .01 & .00 & .00 & .00 \\
\hline$b$ & $\cdots$ & $\cdots$ & $\cdots$ & $\cdots$ & $\cdots$ & $\cdots$ & $\cdots$ & $\cdots$ & .00 & .00 & .00 & .00 \\
\hline 6 & $\cdots$ & $\cdots$ & $\cdots$ & $\cdots$ & $\cdots$ & $\cdots$ & $\cdots$ & $\cdots$ & .00 & .00 & .00 & .00 \\
\hline 7 & $\cdots$ & $\cdots$ & $\cdots$ & $\cdots$ & $\cdots$ & $\cdots$ & $\cdots$ & $\cdots$ & .00 & .00 & .00 & .00 \\
\hline 8 & $\cdots$ & $\cdots$ & $\cdots$ & $\cdots$ & $\cdots$ & $\cdots$ & $\cdots$ & $\cdots$ & .00 & .00 & .00 & .00 \\
\hline 9 & $\cdots$ & $\cdots$ & $\cdots$ & $\cdots$ & $\cdots$ & $\cdots$ & $\cdots$ & $\cdots$ & .00 & .00 & .00 & .00 \\
\hline 10 & $\cdots$ & $\cdots$ & $\cdots$ & $\cdots$ & $\cdots$ & $\cdots$ & $\cdots$ & $\cdots$ & .00 & .00 & .13 & .00 \\
\hline 11 & $\cdots$ & $\cdots$ & $\cdots$ & $\cdots$ & $\cdots$ & $\cdots$ & $\cdots$ & $\cdots$ & .00 & .00 & .04 & .00 \\
\hline 12 & $\cdots$ & $\cdots$ & $\cdots$ & $\cdots$ & $\cdots$ & $\cdots$ & $\cdots$ & $\cdots$ & .00 & .00 & .01 & .00 \\
\hline 13 & $\cdots$ & $\cdots$ & $\cdots$ & $\cdots$ & $\cdots$ & $\cdots$ & $\cdots$ & $\cdots$ & .00 & .00 & .00 & .00 \\
\hline 14 & $\cdots$ & $\cdots$ & $\cdots$ & $\cdots$ & $\cdots$ & $\cdots$ & $\cdots$ & $\cdots$ & .00 & .00 & .00 & .00 \\
\hline 15 & $\cdots$ & $\cdots$ & $\cdots$ & $\cdots$ & $\cdots$ & $\cdots$ & $\cdots$ & $\cdots$ & .00 & .00 & .00 & .00 \\
\hline 16 & $\cdots$ & $\cdots$ & $\cdots$ & $\cdots$ & $\cdots$ & $\cdots$ & $\cdots$ & $\cdots$ & .06 & .00 & .00 & .00 \\
\hline 17 & $\cdots$ & $\cdots$ & $\cdots$ & $\cdots$ & $\cdots$ & $\cdots$ & $\cdots$ & - & .03 & .00 & .00 & .00 \\
\hline 18 & $\cdots$ & $\cdots$ & $\cdots$ & $\cdots$ & $\cdots$ & $\cdots$ & $\cdots$ & e. 20 & .01 & .00 & .00 & .00 \\
\hline 19 & $\cdots$ & $\cdots$ & $\cdots$ & $\cdots$ & $\cdots$ & $\cdots$ & $\cdots$ & .27 & .01 & .00 & .03 & .00 \\
\hline 20 & $\cdots$ & $\cdots$ & $\cdots$ & $\cdots$ & $\cdots$ & $\cdots$ & $\cdots$ & .12 & .03 & .00 & .00 & .00 \\
\hline 21 & $\cdots$ & $\cdots$ & $\cdots$ & $\cdots$ & $\cdots$ & $\cdots$ & $\cdots$ & .00 & .02 & .00 & .00 & .18 \\
\hline 22 & $\cdots$ & $\cdots$ & $\cdots$ & $\cdots$ & $\cdots$ & $\cdots$ & $\cdots$ & .00 & .11 & .00 & .01 & .04 \\
\hline 23 & $\cdots$ & $\cdots$ & $\cdots$ & $\cdots$ & $\cdots$ & $\cdots$ & $\cdots$ & .00 & .01 & .00 & .01 & .00 \\
\hline 24 & $\cdots$ & $\cdots$ & $\cdots$ & $\cdots$ & $\cdots$ & $\cdots$ & $\cdots$ & .00 & .01 & .00 & e. 01 & .00 \\
\hline 25 & $\cdots$ & $\cdots$ & $\cdots$ & $\cdots$ & $\cdots$ & $\cdots$ & $\cdots$ & .00 & .00 & .00 & e. 01 & .00 \\
\hline 26 & $\cdots$ & $\cdots$ & $\cdots$ & $\ldots$ & $\cdots$ & $\cdots$ & $\cdots$ & .00 & .01 & .00 & e. 01 & .00 \\
\hline 27 & $\cdots$ & $\cdots$ & $\cdots$ & $\cdots$ & $\cdots$ & $\cdots$ & $\cdots$ & .00 & .00 & .00 & e. 01 & .00 \\
\hline 28 & $\cdots$ & $\cdots$ & $\cdots$ & $\cdots$ & $\cdots$ & $\cdots$ & $\cdots$ & .14 & .00 & .00 & e. 01 & .00 \\
\hline 29 & $\cdots$ & $\cdots$ & $\cdots$ & $\cdots$ & $\cdots$ & $\cdots$ & $\cdots$ & .00 & .00 & .00 & e. 01 & .00 \\
\hline 30 & $\cdots$ & $\cdots$ & $\cdots$ & $\cdots$ & $\cdots$ & $\cdots$ & $\cdots$ & .00 & .00 & .00 & e. 01 & .00 \\
\hline 31 & $\cdots$ & $\cdots$ & $\cdots$ & $\cdots$ & $\cdots$ & $\cdots$ & $\cdots$ & .12 & $\cdots$ & .00 & .11 & $\cdots$ \\
\hline TOTAL, & $\cdots$ & $\cdots$ & $\cdots$ & $\cdots$ & $\cdots$ & $\cdots$ & $\cdots$ & $\cdots$ & 0.52 & 0.00 & 0.41 & 0.25 \\
\hline MEAN & $\cdots$ & $\cdots$ & $\cdots$ & $\cdots$ & $\cdots$ & $\cdots$ & $\cdots$ & $\cdots$ & .017 & .000 & .013 & .008 \\
\hline MAX & $\cdots$ & $\cdots$ & $\cdots$ & $\cdots$ & $\cdots$ & $\cdots$ & $\cdots$ & $\cdots$ & .20 & .00 & .13 & .18 \\
\hline MIN & $\cdots$ & $\cdots$ & $\cdots$ & $\cdots$ & $\cdots$ & $\cdots$ & $\cdots$ & $\cdots$ & .00 & .00 & .00 & .00 \\
\hline $\mathrm{AC}-\mathrm{FT}$ & $\cdots$ & $\cdots$ & $\cdots$ & $\cdots$ & $\cdots$ & $\cdots$ & $\cdots$ & $\cdots$ & 1.0 & .00 & .8 & .5 \\
\hline
\end{tabular}

e Eslimated 


\section{T-130 DITCH AT MCKAY BYPASS}

SITE NUMBER. - - SW998

STATION IDENTIFICATION. - 395332105124600

LOCATION. - Lat $39^{\circ} 53^{\prime} 32^{\prime \prime}$, long $105^{\circ} 12^{\prime} 46^{\prime \prime}$, in $\mathrm{SW}^{1} / 4 \mathrm{SW}^{1} / 4 \mathrm{sec}, 10, \mathrm{~T} .2 \mathrm{~S}$, R. 70 W., Jefferson

County, Hydrologic Unit 10190003, Rocky Flats Environmental Technology Site, on west Diversion

Ditch downstream from the 130-building complex.

DRAINAGE AREA. - - $0.33 \mathrm{mi}^{2}$.

PERIOD OF RECORD. - MaY 1994 to current year.

GAGE. - Water-stage recorder and Parshall flume with weir plate. Elevation of gage is $6,047 \mathrm{ft}$ above sea level.

REMARKS.--Records poor.

DISCHARGE, CUBIC FEET PER SFCOND, WATER YEAR OCTOBER 1994 TO SFPTEMBER $199 \mathrm{~b}$

\begin{tabular}{|c|c|c|c|c|c|c|c|c|c|c|c|c|}
\hline DAY & $O C T$ & NOV & DEC & JAN & FEB & MAR & $A P R$ & MAY & JUN & JUL & AUG & SEP \\
\hline 1 & .05 & .00 & e. 00 & e. 00 & e. 10 & .00 & .01 & .19 & .16 & .02 & e. 00 & e. 00 \\
\hline 2 & .00 & .00 & e. 00 & e. 00 & e. 02 & .00 & .01 & .17 & e. 66 & .01 & e. 00 & e. 00 \\
\hline 3 & .00 & .03 & e. 00 & e. 00 & .01 & .00 & .01 & .60 & el.2 & .01 & e. 00 & e. 00 \\
\hline 4 & .00 & .19 & e. 00 & e. 00 & .01 & .00 & .00 & .42 & .70 & .01 & e. 00 & e. 00 \\
\hline 5 & .00 & .06 & e. 00 & e. 00 & .01 & .00 & .00 & .20 & .22 & .00 & e. 00 & e. 00 \\
\hline 6 & .00 & .01 & e. 00 & e. 00 & .01 & .00 & .00 & .67 & .05 & .00 & e. 00 & e. 00 \\
\hline 7 & .01 & .00 & e. 00 & e. 00 & .01 & .03 & .01 & .24 & .14 & .00 & e. 00 & e.00 \\
\hline 8 & .00 & .24 & e. 00 & e. 00 & .01 & .02 & .00 & .41 & 1.3 & .00 & e. 00 & e. 00 \\
\hline 9 & .00 & .05 & e. 00 & e. 00 & .01 & .01 & .04 & .58 & 2.3 & .00 & e. 00 & e. 11 \\
\hline 10 & .00 & .02 & e. 00 & e. 00 & e. 01 & .00 & .22 & .74 & .11 & .00 & e. 00 & e. 06 \\
\hline 11 & .00 & .01 & e. 00 & e.00 & e. 01 & .00 & .18 & .61 & .06 & .00 & .00 & e. 04 \\
\hline 12. & .00 & .00 & e. 00 & e. 00 & e. 01 & .01 & .11 & .29 & .03 & .00 & .00 & e. 00 \\
\hline 13 & .00 & .00 & e. 00 & e. 00 & e. 70 & .01 & .03 & .06 & .02 & .00 & .00 & e. 00 \\
\hline 14 & .00 & .18 & e. 00 & e. 00 & .26 & .00 & .02 & .02 & .01 & .03 & .06 & e. 00 \\
\hline 15 & .02 & .08 & e. 00 & e. 00 & .03 & .01 & .01 & .01 & .01 & .01 & .01 & e. 00 \\
\hline 16 & .04 & .04 & e. 00 & e. 00 & .03 & .04 & .13 & e. 40 & .00 & .01 & .00 & e. 00 \\
\hline 17 & .45 & .03 & e. 00 & e. 00 & .10 & .02 & .50 & e10 & .17 & .00 & .00 & e. 00 \\
\hline 18 & .01 & .01 & e. 00 & e. 00 & .05 & .02 & .46 & .65 & .02 & .00 & .32 & e. 33 \\
\hline 19 & .00 & .01 & e. 00 & e. 00 & .03 & .02 & .92 & .30 & .00 & .07 & .19 & e. 31 \\
\hline 20 & .00 & .01 & e. 00 & e. 00 & .02 & .02 & .62 & .08 & .00 & .01 & .14 & e. 50 \\
\hline 21 & .00 & .01 & e. 00 & e. 00 & .01 & .02 & 1.3 & .05 & .00 & .01 & .14 & e. 55 \\
\hline 22 & .00 & .00 & e. 00 & .00 & .01 & .02 & 1.3 & .06 & .00 & .00 & .06 & e. 40 \\
\hline 23 & .00 & e. 63 & e. 00 & .00 & .00 & .02 & 1.2 & e1.6 & .04 & .00 & .00 & e. 27 \\
\hline 24 & .00 & .00 & e. 00 & .00 & .00 & .03 & .91 & 2.2 & .02 & .00 & .00 & e.16 \\
\hline 25 & .00 & .00 & e. 00 & .00 & .00 & .03 & e. 59 & .69 & e. 02 & .00 & .00 & e. 16 \\
\hline 26 & .00 & .00 & e. 00 & .00 & .00 & .15 & e2. 3 & e. 70 & e. 02 & .00 & .00 & e. 13 \\
\hline 27 & .00 & .00 & e. 00 & .00 & .00 & .05 & el. 6 & $c 2.2$ & e. 02 & .00 & .00 & e. 12 \\
\hline 28 & .00 & e. 00 & e. 00 & .00 & .01 & .19 & e. 75 & t 1.5 & e. 92 & .00 & .00 & e. 11 \\
\hline 29 & .00 & e. 00 & e. 00 & .15 & $\cdots$ & .10 & e1.8 & 1.1 & .60 & .00 & .00 & e. 30 \\
\hline 30 & .00 & e. 00 & e. 00 & .21 & $\cdots$ & .07 & e1.5 & .68 & .04 & .00 & .00 & e. 50 \\
\hline 31 & .00 & $\cdots$ & e. 00 & e. 21 & $\cdots$ & .02 & $\cdots$ & .33 & $\cdots$ & .01 & .00 & $\cdots$ \\
\hline TOTAL & 0.58 & 1.61 & 0.00 & 0.57 & 1.47 & 0.91 & 16.53 & 27.75 & 8.84 & 0.20 & 0.92 & 4.05 \\
\hline MEAN & .019 & .054 & .000 & .018 & .052 & .029 & .55 & .90 & .29 & .006 & .030 & .13 \\
\hline MAX & .45 & .63 & .00 & .21 & .70 & .19 & 2.3 & 10 & 2.3 & .07 & .32 & .55 \\
\hline MIN & .00 & .00 & .00 & .00 & .00 & .00 & .00 & .01 & .00 & .00 & .00 & .00 \\
\hline$A C-F T$ & 1.2 & 3.2 & .00 & 1.1 & 2.9 & 1.8 & 33 & 55 & 18 & .4 & 1.8 & 8.0 \\
\hline
\end{tabular}

STATISTICS OF MONTHL, MEAN DATA FOR WATER YEARS 1994 - 1995, BY WATER YEAR (WWY)

\begin{tabular}{|c|c|c|c|c|c|c|c|c|c|c|c|c|}
\hline MEAN & .019 & .054 & .000 & .018 & $.0 ๖ 2$ & .029 & .55 & .90 & .16 & .003 & .021 & .072 \\
\hline MAX & .019 & .054 & .000 & .018 & .052 & .029 & .55 & .90 & .29 & .006 & .030 & .13 \\
\hline (WY) & 1995 & 1995 & 1995 & 1995 & 1995 & 1995 & 1995 & 1995 & 1995 & 1995 & 1995 & 1995 \\
\hline MIN & .019 & .054 & .000 & .018 & .052 & .029 & .55 & .90 & .017 & .000 & .013 & .008 \\
\hline (WY) & 1995 & 1995 & 1995 & 1995 & 1995 & 1995 & 1995 & 1995 & 1994 & 1994 & 1994 & 1994 \\
\hline
\end{tabular}

SUMMARY STATISTICS

FOR 1995 WATER YEAR

WATER YEARS 1994 - 1995

ANNUAL TUTAL

HIGHEST ANNUAL MEAN

LOWEST ANNUAL MEAN

HIGHEST DAILY MEAN

LOWEST DAILY MEAN

ANNUAL SEVEN-DAY MINIMUM

ANNUAL RUNOFF (AC-FT)

10 PERCENT EXCEEDS

50 PERCENT EXCEEDS

90 PERCENT EXCEEDS

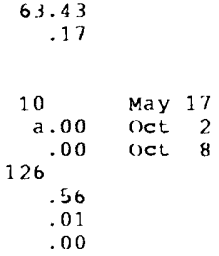

a No flow many days

e Estimated 


\section{WATER-QUALITY AND SUSPENDED-SEDIMENT DATA}

The following abbreviations are used in tables 41 to 44 .

mmddyy is month/day/year;

hhmm is hours (0 to 24$)$ and minutes ( 0 to 60$)$;

cfs is cubic feet per second; $\mathrm{ft}^{3} / \mathrm{s}$ is cubic feet per second;

$\mu \mathrm{S} / \mathrm{cm}$ at $25^{\circ} \mathrm{C}$ is microsiemens per centimeter at 25 degrees Celsius;

${ }^{\circ} \mathrm{C}$ is degrees Celsius;

$\mu \mathrm{g} / \mathrm{L}$ is micrograms per liter;

$\mathrm{mg} / \mathrm{L}$ is milligrams per liter

$\mathrm{pCi} / \mathrm{L}$ is picocuries per liter;

--- is a symbol used in place of daily mean discharge for periods of missing record or periods prior to gaging-station activation. 


\section{Table 41.-- Water-quality data, water year 1994}

(The source for teported concentrations is the Rocky Flats Environmental Database (RFEDS), which does not specify the number of significant figures associated with analytical determinations; consequentiy, all concentrations in this table are reported to three decimal places. Laboratory-determined counting errors associated with radionuclide determinations are shown as plus or minus qualifiers. For radionuclides the counting error is used to qualify the data and no other qualifiers are shown. Reported concentrations for all constituents other than ladionuclides may show qualifiers to the right of the reported concentration as foliows: B (for metals), reported value is less than method detection level but greater than instrument detection level. For consord values for constituents other than radionuclides, the censored 1 imit is normally reported as the RFED detection 1 imit. In some cases for metals determinations the RFEDS detection limit field may contaln the the contractor-required detection limit; for these cases the censoring limit is the RFEDS reported value; <, less than]

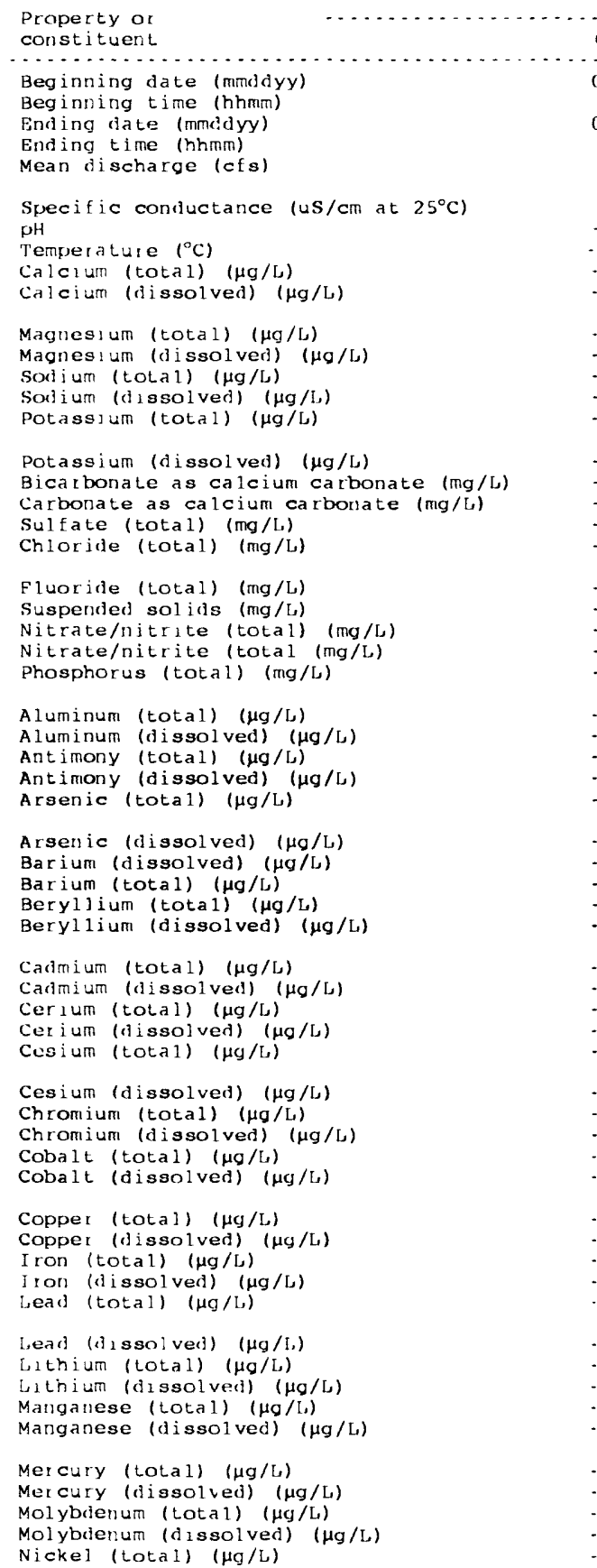

site number (fig. 1)

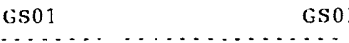

$\begin{array}{ccc}05 / 13 / 94 & 07 / 23 / 94 & \ldots \ldots \ldots \\ 0140 & 1440 & 10 / 07 / 93 \\ 05 / 14 / 94 & 07 / 28 / 94 & 1825 \\ 0622 & 2358 & 10 / 07 / 93 \\ 0.130 & 1.500 & \cdots \\ \cdots & \cdots & \cdots \\ \cdots & \cdots & \cdots\end{array}$

GS07

$\cdots$

$\cdots$

$\begin{array}{lll}\ldots & \ldots & 22,200.000 \\ & & 20,500.000\end{array}$

$\begin{array}{lll}\ldots & \ldots & 5,620.000\end{array}$

$\begin{array}{lll}\ldots & \ldots & 5,620.000 \\ & & 5,090.000\end{array}$

$13,800.000$

$\begin{array}{lll}\ldots & \ldots & 13,300.000\end{array}$

$5,340.000$

$4,740.000$

$<5.000$

$<1.000$

$<64.000$
$<\quad 23.000$

$10 / 09 / 93$

1210

$\cdots$

$\cdots$

$\cdots$

$\cdots$

$\cdots$

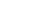

40.600

56.300

56.300

$10 / 09 / 93$

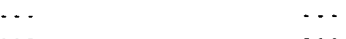

$\begin{array}{ll}\cdots & \ldots \\ \cdots & \ldots\end{array}$

...

...

$\cdots+\cdots$

$\begin{array}{ll}\cdots & \cdots\end{array}$

$\cdots-\cdots$

$3,110.000$

40.600

$<24.000$

$<23.000$

$<94.000$

$<64.000$

56.300

$\begin{array}{ll}\cdots & \cdots\end{array}$

$\begin{array}{ll}\cdots & \cdots \\ \cdots & \cdots\end{array}$

$\begin{array}{ll}\cdots & \cdots \\ \cdots & \cdots\end{array}$

89.200

$<1.000$

$<1.000$

4.000
$<5.000$

-..

8.900

4. 500

7.500

$<.000$

...

$\ldots$

$78,400.000$

$79,000.000$

$16,400.000$

$16,700.000$

$42,600.000$

$42,200.000$

$2,430.000$

2.680 .000

$<5.000$

$<1.000$

$<64.000$
$<23.000$

$\cdots$

$\cdots$

-.

$\cdots$

$\cdots$

$<8.000$

8.000
4.300

$\begin{array}{ll}\cdots & \cdots \\ \cdots & \cdots\end{array}$

$\begin{array}{ll}\cdots & \cdots \\ \cdots & \cdots\end{array}$

$\begin{array}{ll}\cdots & \cdots\end{array}$

.070 .000

60.900

$<67.000$

53.500

$\cdots$

-

75.000
$<4.400$

2.000

314.000

1.300

124.000

…

706.000

53.500

24.000

$<23.000$

$<94.000$

$<64.000$

B $\quad 124.000$

133.000

$<1.000$

$<1.000$

$<4.000$

$<5.000$

$\cdots$

7.000

$\begin{array}{ll}\cdots & \cdots\end{array}$

...

...

$<6.000$

$<9.000$

$<3.000$

$<.000$

$<.000$

...

$<8.000$

4.100

4.100
859.000

6.800
6.000

$<67.000$

$\begin{array}{ll}\cdots & \cdots \\ \cdots & \cdots\end{array}$

$+\cdots$

..

$<75.000$

12.400

11.000

136.000

$<1.000$

-..

$<6.000$

$<9.000$ 
Table 41.-- Water-quality data, water year 1994--continued

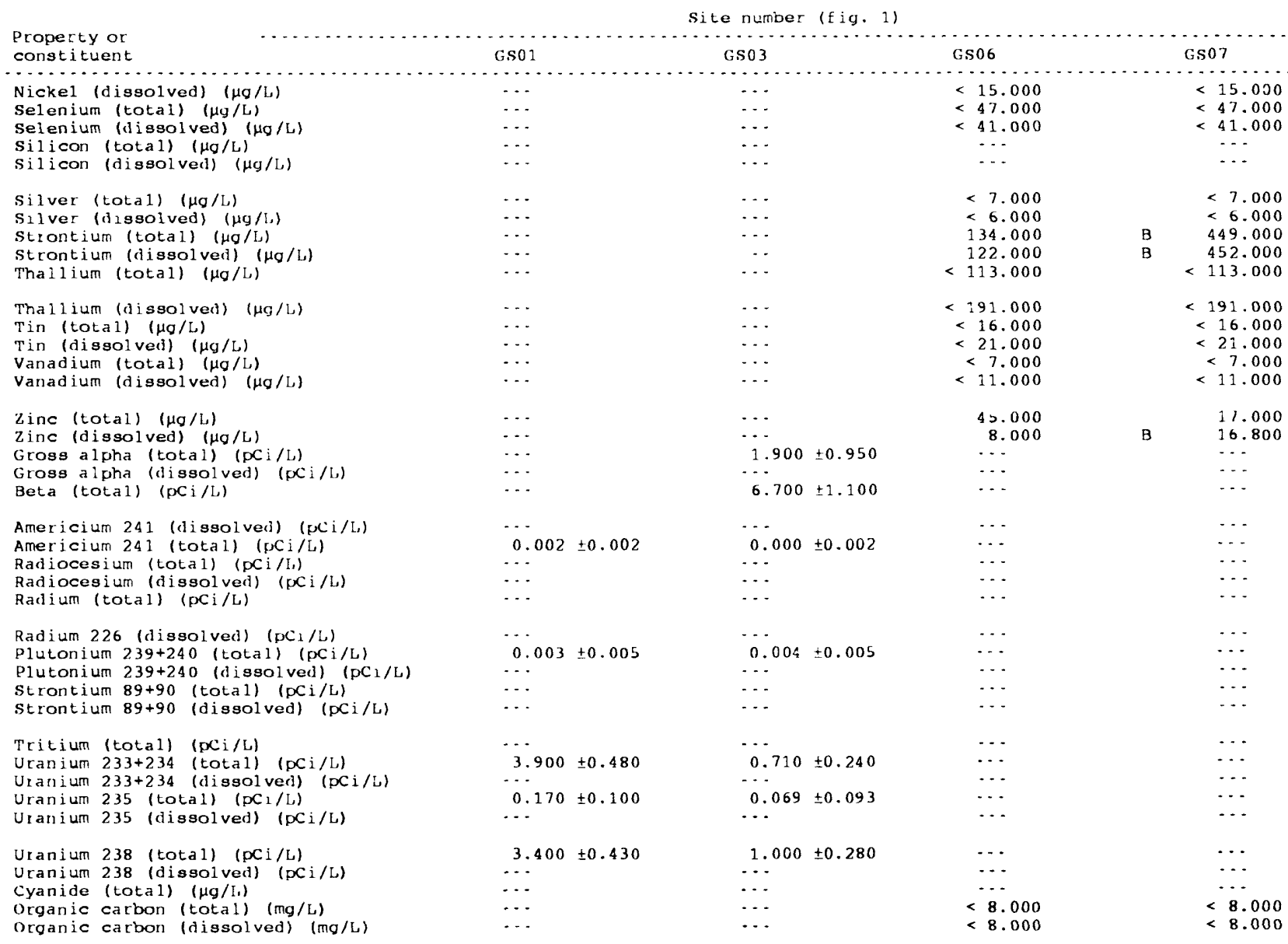


Table 41.-- Water-quality data, water year 1994--continued

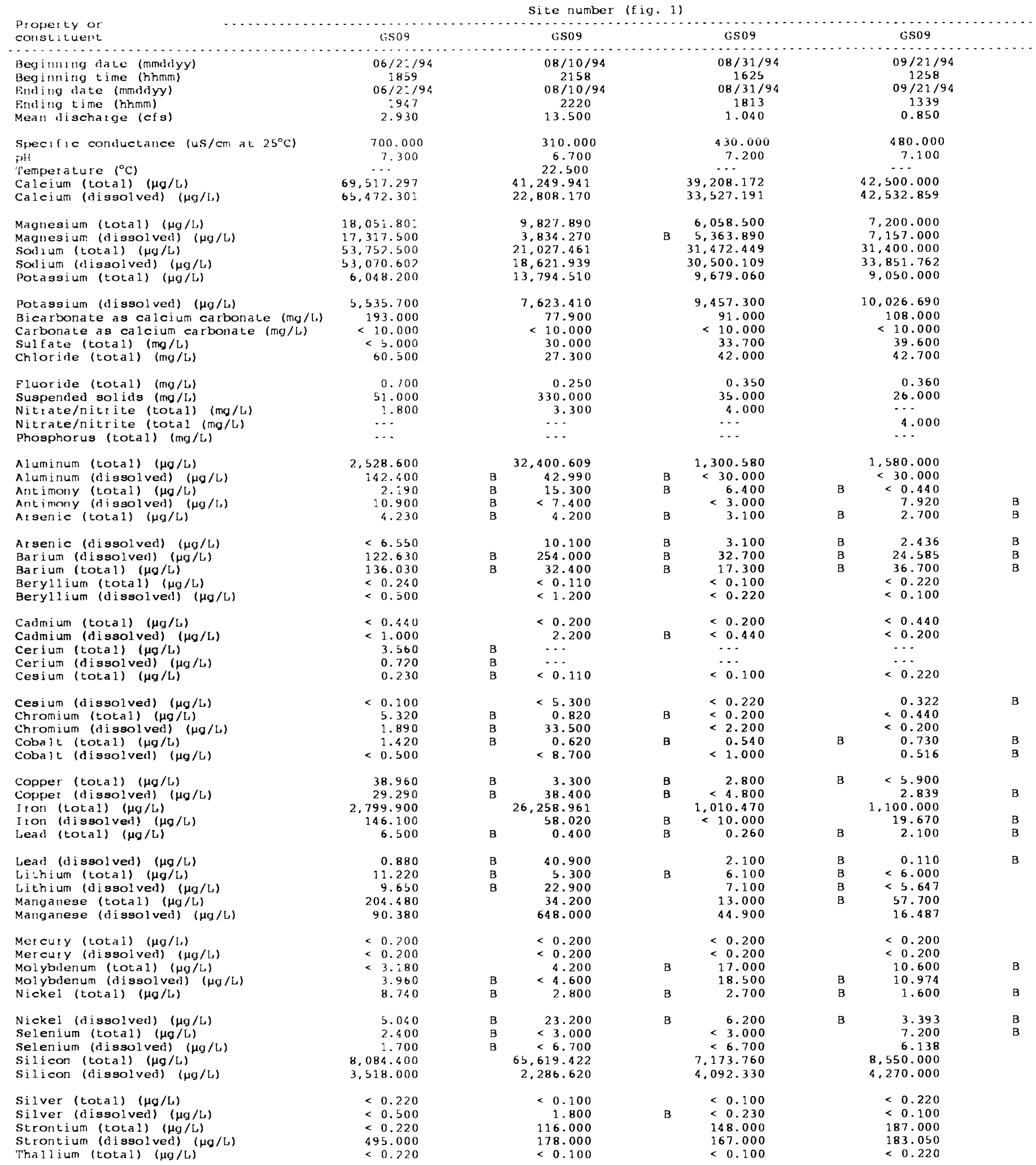


Table 41.-- Water-quality data, water year 1994--continued

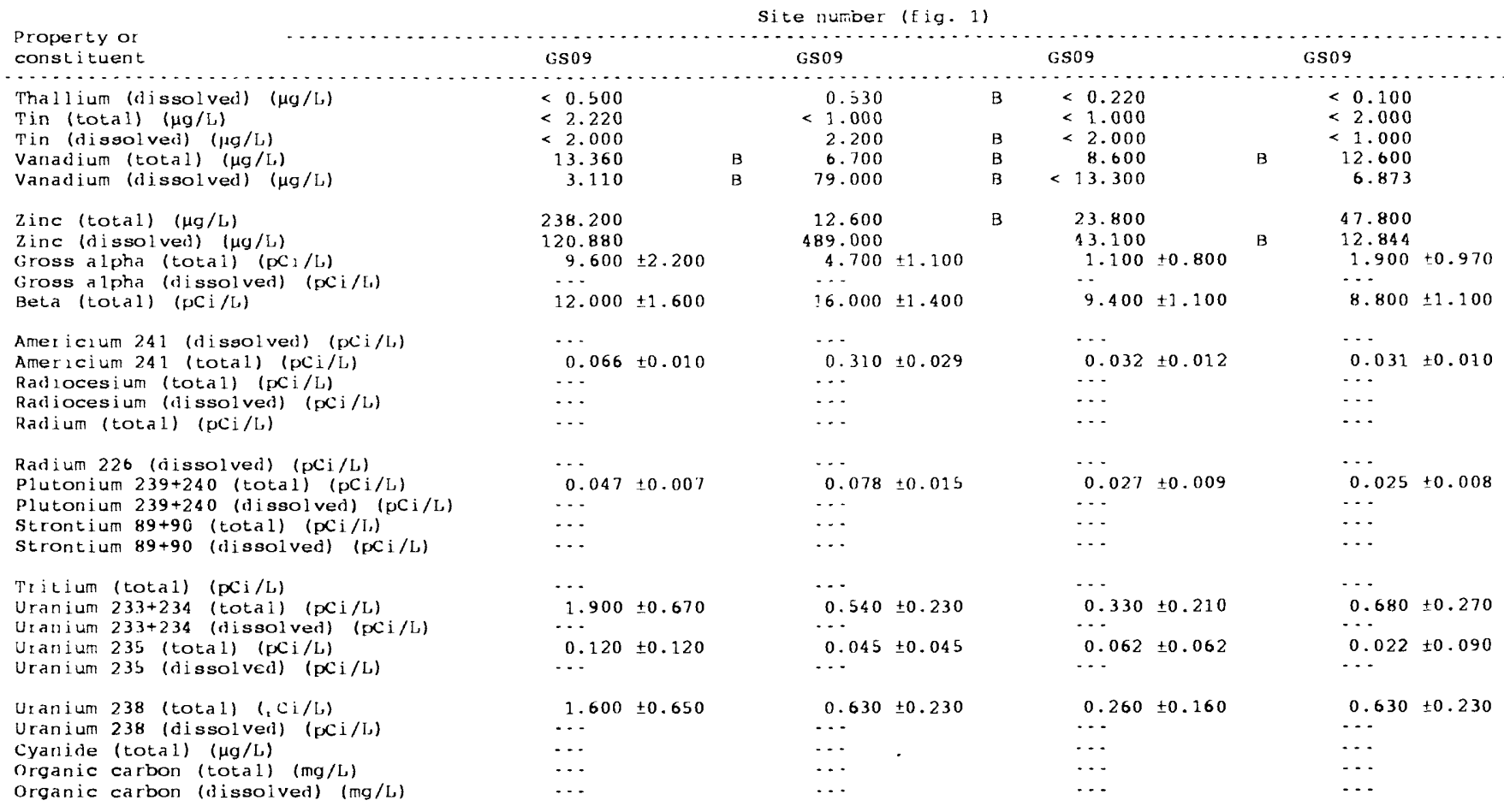


Table 41.-- Water-quality data, water year 1994--continued

\begin{tabular}{|c|c|c|c|c|c|c|c|c|}
\hline $\begin{array}{l}\text { Property or } \\
\text { constituent }\end{array}$ & $\operatorname{Gs} 10$ & \multicolumn{3}{|c|}{ Site number (fig. 1) } & GS10 & & $\operatorname{cs} 10$ & \\
\hline $\begin{array}{l}\text { Beginning date (mmidyy) } \\
\text { Beginning time (hhmm) } \\
\text { Ending date (mmddyy) } \\
\text { Ending time (hhmm) } \\
\text { Mean discharge (cfs) }\end{array}$ & $\begin{array}{l}03 / 28 / 94 \\
1219 \\
05 / 28 / 94 \\
: 236 \\
3.250\end{array}$ & & $\begin{array}{l}05 / 31 / 94 \\
1759 \\
05 / 31 / 94 \\
1811 \\
5.620\end{array}$ & & $\begin{array}{l}06 / 20 / 94 \\
2031 \\
06 / 20 / 94 \\
2228 \\
0.220\end{array}$ & & $\begin{array}{l}06 / 21 / 94 \\
1838 \\
06 / 21 / 94 \\
1856 \\
4.330\end{array}$ & \\
\hline $\begin{array}{l}\left.\text { Specific conductance (uS/cm at } 25^{\circ} \mathrm{C}\right) \\
\text { pH } \\
\text { Temperature }\left({ }^{\circ} \mathrm{C}\right) \\
\text { Calcium (total) }(\mu \mathrm{g} / \mathrm{L}) \\
\text { Calcium (dissolved) }(\mu \mathrm{g} / \mathrm{L})\end{array}$ & $\begin{array}{c}\cdots \\
\cdots \\
60,100.000 \\
33,400.000\end{array}$ & & $\begin{array}{c}\cdots \\
\cdots \\
48,800.000 \\
21,800.000\end{array}$ & & $\begin{array}{c}468.000 \\
7.300 \\
\cdots . \\
35,366.898 \\
32,248.100\end{array}$ & & $\begin{array}{r}320.000 \\
7.400 \\
17.500 \\
65.301 .199 \\
30.142 .301\end{array}$ & \\
\hline $\begin{array}{l}\text { Magriesium (total) ( } \mu \mathrm{g} / \mathrm{L}) \\
\text { Magnesium (dissolved) }(\mu \mathrm{g} / \mathrm{L}) \\
\text { Sodium (total) }(\mu \mathrm{g} / \mathrm{L}) \\
\text { Sodium (dissolved) }(\mu \mathrm{g} / \mathrm{L}) \\
\text { Potasstum (total) }(\mu \mathrm{g} / \mathrm{L})\end{array}$ & $\begin{array}{r}13,500.000 \\
6,640.000 \\
29,000.000 \\
25,900.000 \\
8,600.000\end{array}$ & & $\begin{array}{r}10.300 .000 \\
4.070 .000 \\
15,700.000 \\
14.600 .000 \\
7.190 .000\end{array}$ & B & $\begin{array}{r}7,520.700 \\
6,421.500 \\
26,927.400 \\
28,700.000 \\
8,917.400\end{array}$ & & $\begin{array}{r}16,228.100 \\
6,064.400 \\
18,435.000 \\
19,882.100 \\
12,733.300\end{array}$ & \\
\hline $\begin{array}{l}\text { Potassium (dissolved) ( } \mu \mathrm{g} / \mathrm{L}) \\
\text { Bicarbonate as calcium carbonate (mg/L) } \\
\text { carbonate as calcium carbonate (mg/L) } \\
\text { Sulfate (total) (mg/L) } \\
\text { Chloride (total) (mg/L) }\end{array}$ & $\begin{array}{r}4.080 .000 \\
\quad 93.900 \\
<\quad 10.000 \\
49.000 \\
48.300\end{array}$ & B & $\begin{array}{r}3.090 .000 \\
\quad 72.900 \\
<\quad 10.000 \\
28.300 \\
18.900\end{array}$ & B & $\begin{array}{r}8.369 .300 \\
65.300 \\
<\quad 10.000 \\
61.000 \\
36.700\end{array}$ & & $\begin{array}{r}3.848 .200 \\
126.000 \\
<\quad 10.000 \\
38.600 \\
26.500\end{array}$ & B \\
\hline $\begin{array}{l}\text { Fluoride (total) (mg/L) } \\
\text { Suspended solids (mg/L) } \\
\text { Nitrate/nitrite (total) (mg/L) } \\
\text { Nitrate/nitrite (total (mg/L) } \\
\text { Phosphorus (tota) (mg/L) }\end{array}$ & $\begin{array}{c}0.370 \\
1,200.000 \\
\cdots \\
\cdots\end{array}$ & & $\begin{array}{r}0.260 \\
1.500 .000 \\
1.000 \\
\cdots\end{array}$ & & $\begin{array}{l}0.350 \\
180.000 \\
5.700 \\
\cdots\end{array}$ & & $\begin{array}{r}0.350 \\
1.400 .000 \\
1.500 \\
\cdots\end{array}$ & \\
\hline 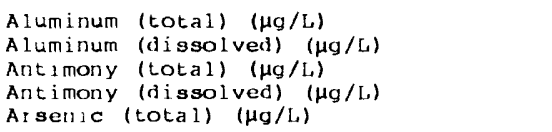 & $\begin{array}{r}15.000 .000 \\
<50.000 \\
6.100 \\
13.400 \\
17.100\end{array}$ & $\begin{array}{l}\text { B } \\
\text { B } \\
B\end{array}$ & $\begin{array}{r}13.100 .000 \\
<50.000 \\
13.100 \\
20.900 \\
26.600\end{array}$ & $\begin{array}{l}\text { B } \\
\text { B } \\
\text { B }\end{array}$ & $\begin{array}{r}8,539.200 \\
<50.000 \\
9.380 \\
19.930 \\
5.010\end{array}$ & $\begin{array}{l}\text { B } \\
\text { B } \\
B\end{array}$ & $\begin{array}{r}58,933.699 \\
<50.000 \\
20.590 \\
48.420 \\
10.110\end{array}$ & $\begin{array}{l}B \\
B \\
B\end{array}$ \\
\hline 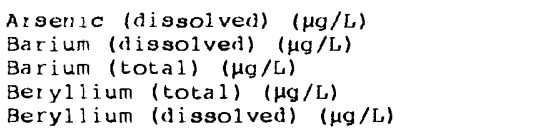 & $\begin{array}{r}2.000 \\
61.200 \\
424.000 \\
1.300 \\
<\quad 0.100\end{array}$ & $\begin{array}{l}\text { B } \\
\text { B } \\
\text { B } \\
\text { B }\end{array}$ & $\begin{array}{r}2.100 \\
39.800 \\
487.000 \\
2.300 \\
<\quad 0.100\end{array}$ & $\begin{array}{l}B \\
B \\
B \\
B\end{array}$ & $\begin{array}{r}<6.130 \\
30.590 \\
75.130 \\
<\quad 0.220 \\
<0.100\end{array}$ & $\begin{array}{l}\text { B } \\
\text { B }\end{array}$ & $\begin{array}{r}<2.970 \\
55.150 \\
459.640 \\
<0.780 \\
<0.100\end{array}$ & B \\
\hline $\begin{array}{l}\text { Cadmium (total) }(\mu \mathrm{g} / \mathrm{L}) \\
\text { Cadmium (dissolved) }(\mu \mathrm{g} / \mathrm{L}) \\
\text { Cerium (total) }(\mu \mathrm{g} / \mathrm{L}) \\
\text { Cerium (dissolved) }(\mu \mathrm{g} / \mathrm{L}) \\
\text { Cesium (total) }(\mu \mathrm{g} / \mathrm{L})\end{array}$ & $\begin{array}{r}<\quad 6.200 \\
<\quad 0.200 \\
97.200 \\
<\quad 0.100 \\
5.100\end{array}$ & B & $\begin{array}{r}<4.000 \\
<0.200 \\
124.000 \\
0.580 \\
5.900\end{array}$ & $\begin{array}{l}\text { B } \\
\text { B }\end{array}$ & $\begin{array}{r}0.480 \\
<0.200 \\
11.280 \\
0.170 \\
0.930\end{array}$ & $\begin{array}{l}B \\
B \\
B\end{array}$ & $\begin{array}{r}3.080 \\
<0.200 \\
95.440 \\
0.120 \\
6.630\end{array}$ & $\begin{array}{l}B \\
B\end{array}$ \\
\hline 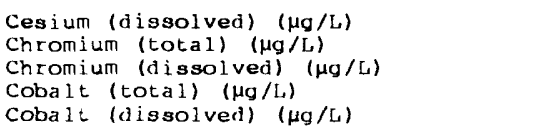 & $\begin{array}{r}<0.100 \\
47.400 \\
0.690 \\
11.200 \\
0.310\end{array}$ & $\begin{array}{l}B \\
B \\
B \\
B\end{array}$ & $\begin{array}{r}0.100 \\
59.300 \\
0.680 \\
14.700 \\
0.200\end{array}$ & $\begin{array}{l}B \\
B \\
B \\
B\end{array}$ & $\begin{array}{r}0.100 \\
6.850 \\
0.770 \\
1.970 \\
0.290\end{array}$ & $\begin{array}{l}\mathrm{B} \\
\mathrm{B} \\
\mathrm{B} \\
\mathrm{B}\end{array}$ & $\begin{array}{r}0.100 \\
31.870 \\
0.860 \\
9.380 \\
0.110\end{array}$ & $\begin{array}{l}\text { B } \\
\text { B } \\
\text { B }\end{array}$ \\
\hline $\begin{array}{l}\text { Copper (total) }(\mu \mathrm{g} / \mathrm{L}) \\
\text { Copper (dissolved) }(\mu \mathrm{g} / \mathrm{L}) \\
\text { I ron (total) ( } \mu \mathrm{g} / \mathrm{L}) \\
\text { I ron (dissolved) ( }(\mu \mathrm{g} / \mathrm{L}) \\
\text { Lear (total) }(\mu \mathrm{g} / \mathrm{L})\end{array}$ & $\begin{array}{r}76.600 \\
6.200 \\
20,000.000 \\
<20.000 \\
94.700\end{array}$ & $\begin{array}{l}\text { B } \\
\text { B }\end{array}$ & $\begin{array}{r}90.300 \\
<3.200 \\
15,500.000 \\
40.600 \\
112.000\end{array}$ & B & $\begin{array}{r}9.260 \\
2.540 \\
6,349.700 \\
<\quad 20.000 \\
8.810\end{array}$ & $\begin{array}{l}\text { B } \\
\text { B }\end{array}$ & $\begin{array}{r}38.180 \\
6.760 \\
49.953 .000 \\
<20.000 \\
70.390\end{array}$ & $\begin{array}{l}\text { B } \\
\text { B }\end{array}$ \\
\hline 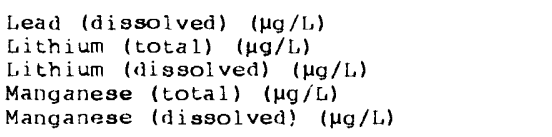 & $\begin{array}{r}<0.100 \\
<\quad 28.300 \\
3.900 \\
1.580 .000 \\
52.700\end{array}$ & B & $\begin{array}{r}0.180 \\
<30.200 \\
2.200 \\
1.690 .000 \\
31.300\end{array}$ & B & $\begin{array}{r}0.240 \\
15.080 \\
13.170 \\
115.760 \\
13.490\end{array}$ & $\begin{array}{l}B \\
B\end{array}$ & $\begin{array}{r}0.180 \\
18.490 \\
3.330 \\
753.090 \\
29.290\end{array}$ & $\begin{array}{l}\text { B } \\
\text { B } \\
\text { B }\end{array}$ \\
\hline 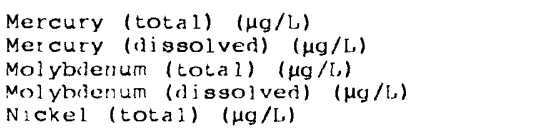 & $\begin{array}{r}<\quad 0.200 \\
<\quad 0.200 \\
4.300 \\
1.800 \\
19.400\end{array}$ & $\begin{array}{l}B \\
B \\
B\end{array}$ & $\begin{array}{r}<0.200 \\
<0.200 \\
3.600 \\
1.010 \\
28.300\end{array}$ & $\begin{array}{l}B \\
B \\
B\end{array}$ & $\begin{aligned}< & 0.200 \\
< & 0.200 \\
& 6.810 \\
& 7.460 \\
& 7.110\end{aligned}$ & $\begin{array}{l}\text { B } \\
\text { B } \\
\text { B }\end{array}$ & $\begin{array}{r}<\quad 0.200 \\
<\quad 0.200 \\
<\quad 3.050 \\
1.360 \\
24.480\end{array}$ & $\begin{array}{l}\mathrm{B} \\
\mathrm{B}\end{array}$ \\
\hline $\begin{array}{l}\text { Nickel (dissolved) }(\mu \mathrm{g} / \mathrm{L}) \\
\text { Selenium (total) }(\mu \mathrm{g} / \mathrm{L}) \\
\text { Selenium (dissolved) }(\mu \mathrm{g} / \mathrm{L}) \\
\text { Silicon (total) ( } \mu \mathrm{g} / \mathrm{L}) \\
\text { Silicon (dissolved) ( } \mu \mathrm{g} / \mathrm{L})\end{array}$ & $\begin{array}{r}2.200 \\
<22.200 \\
<3.000 \\
29.100 .000 \\
2.050 .000\end{array}$ & B & $\begin{array}{r}1.700 \\
<22.200 \\
23.000 \\
26,500.000 \\
1.490 .000\end{array}$ & B & $\begin{array}{r}1.280 \\
<2.200 \\
<1.000 \\
20,828.199 \\
3.725 .300\end{array}$ & B & $\begin{array}{r}1.440 \\
2.300 \\
\times 1.000 \\
78.641 .898 \\
1.913 .300\end{array}$ & $\begin{array}{l}B \\
B\end{array}$ \\
\hline $\begin{array}{l}\text { Silver (total) }(\mu \mathrm{g} / \mathrm{L}) \\
\text { Silver (dissolved) ( } \mu \mathrm{g} / \mathrm{L}) \\
\text { Strontium (total) }(\mu \mathrm{g} / \mathrm{L}) \\
\text { Strontium (dissolved) }(\mu \mathrm{g} / \mathrm{L}) \\
\text { Thallium (total) ( } \mu \mathrm{g} / \mathrm{L})\end{array}$ & $\begin{array}{l}<3.000 \\
<\quad 0.100 \\
343.000 \\
224.000 \\
<\quad 1.100\end{array}$ & & $\begin{array}{l}<3.000 \\
<\quad 0.100 \\
283.000 \\
132.000 \\
<\quad 1.100\end{array}$ & & $\begin{array}{l}<\quad 0.420 \\
<\quad 0.100 \\
175.580 \\
158.000 \\
<\quad 0.220\end{array}$ & & $\begin{array}{r}3.760 \\
<\quad 0.100 \\
253.220 \\
168.000 \\
<\quad 0.940\end{array}$ & B \\
\hline
\end{tabular}


Table 41.-- Water-quality data, water year 1994--continued

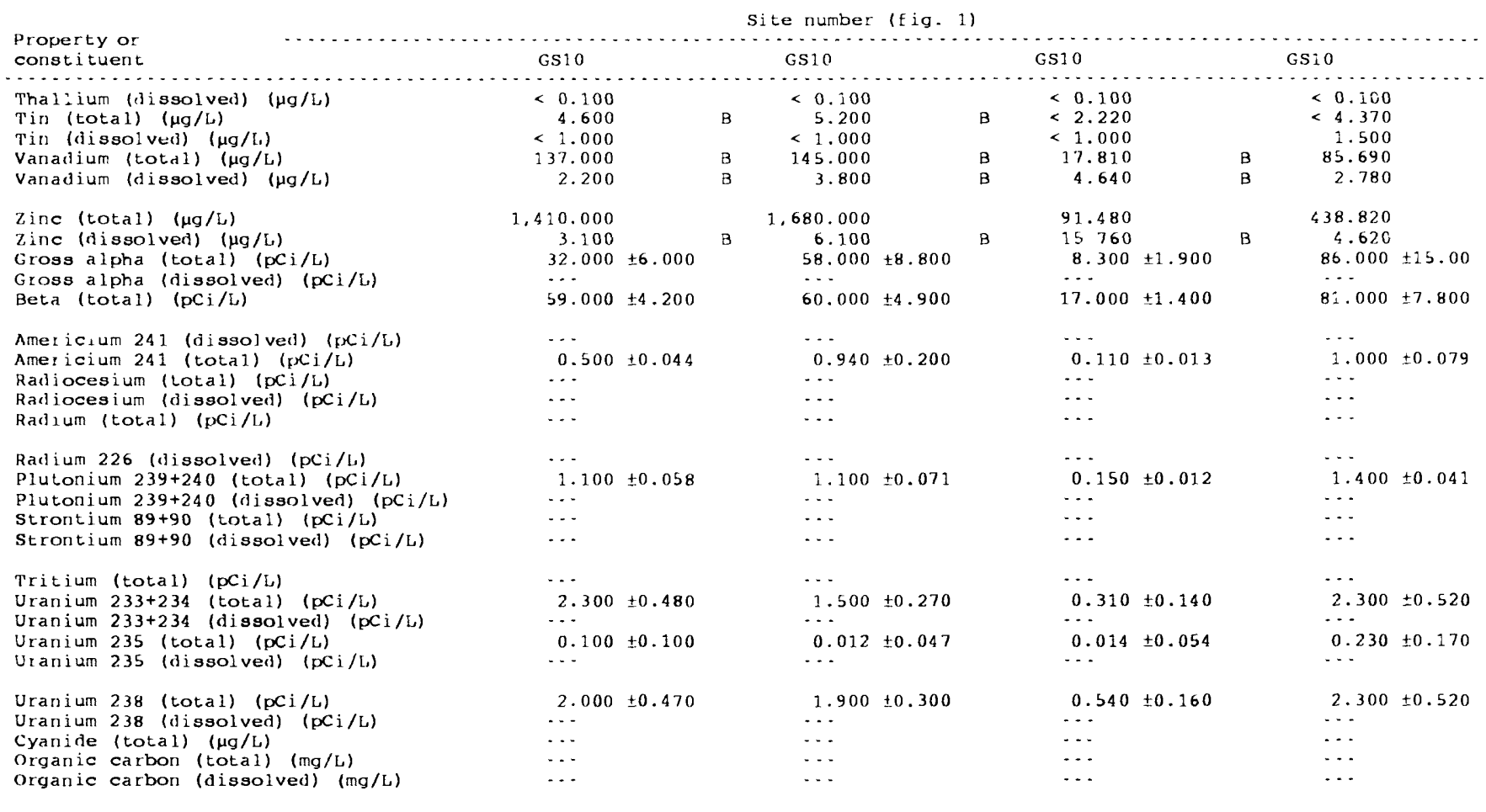


Table 41.-- Water-quality data, water year 1994--continued

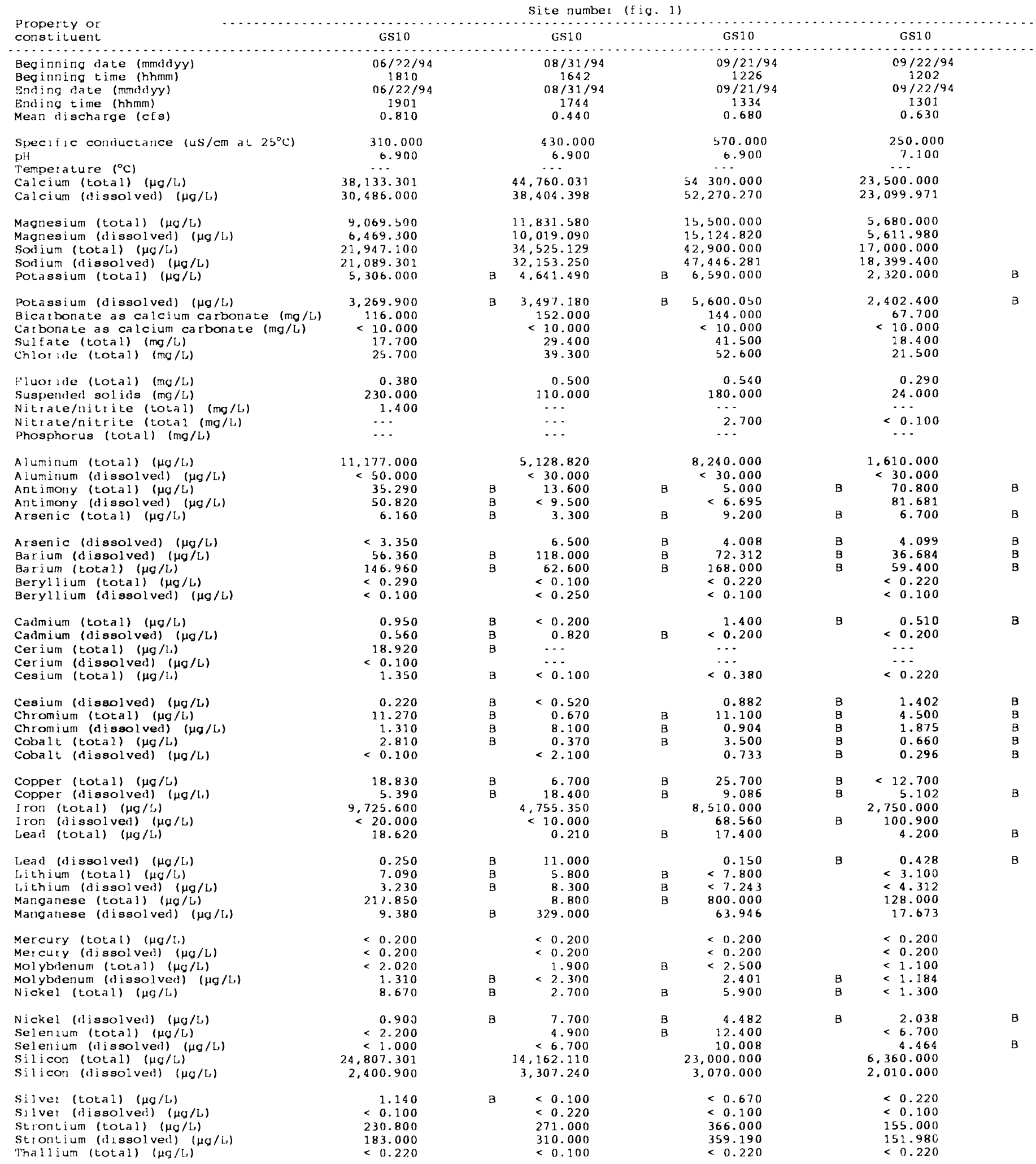


Table 41.-- Water-quality data, water year 1994--continued

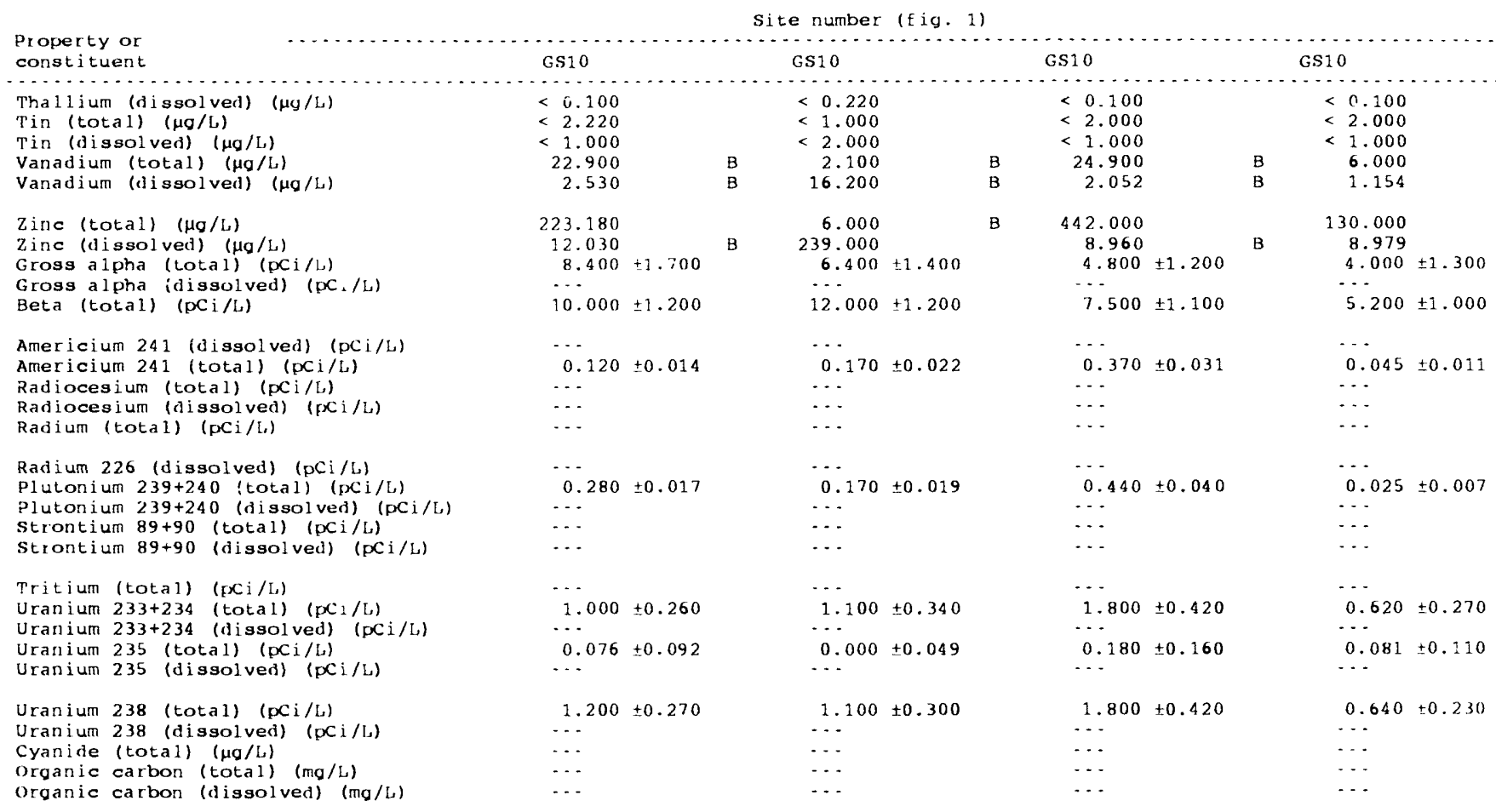


Table 41.-- Water-quality data, water year 1994--continued

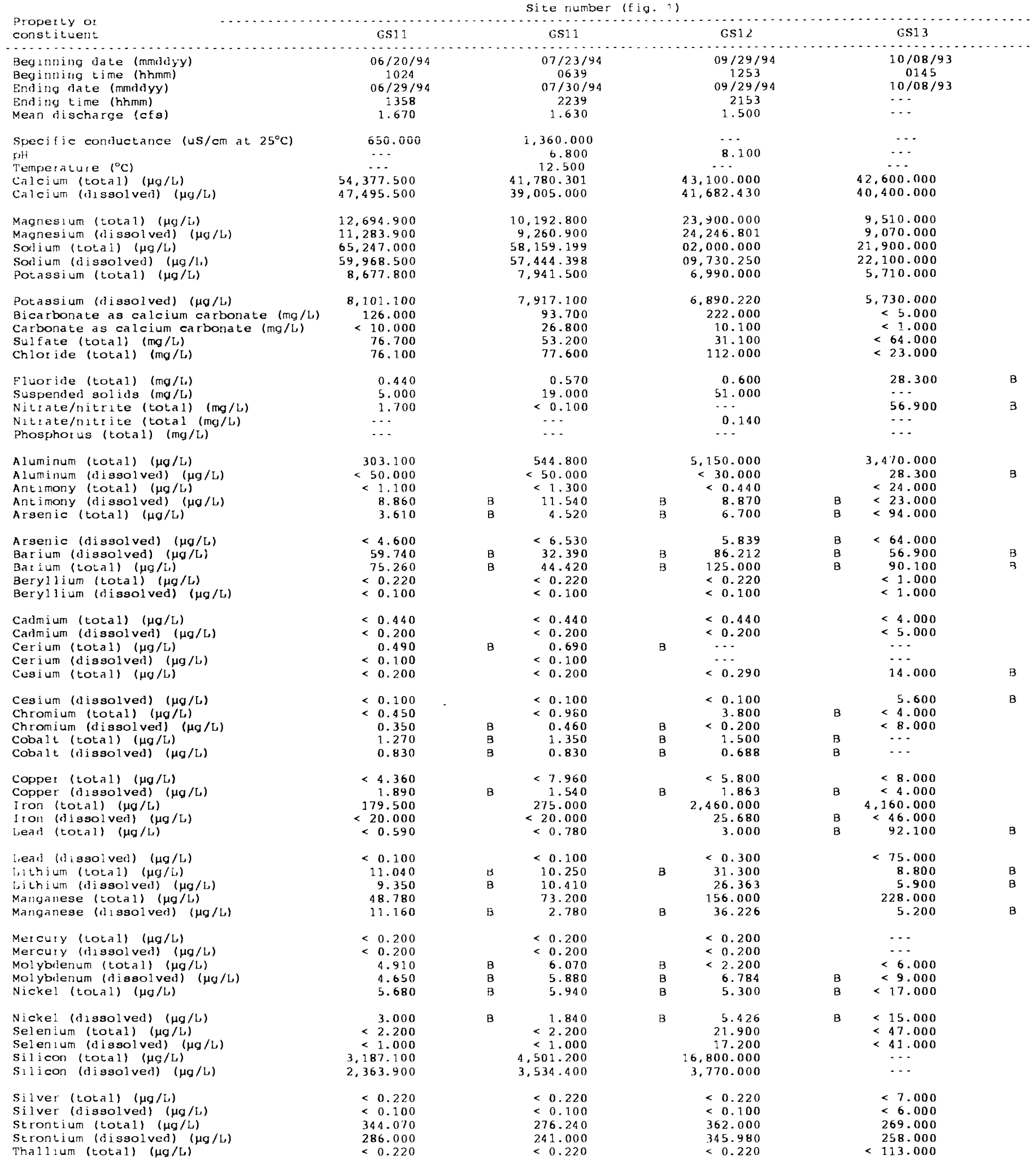


Table 41.-- Water-quality data, water year 1994--continued

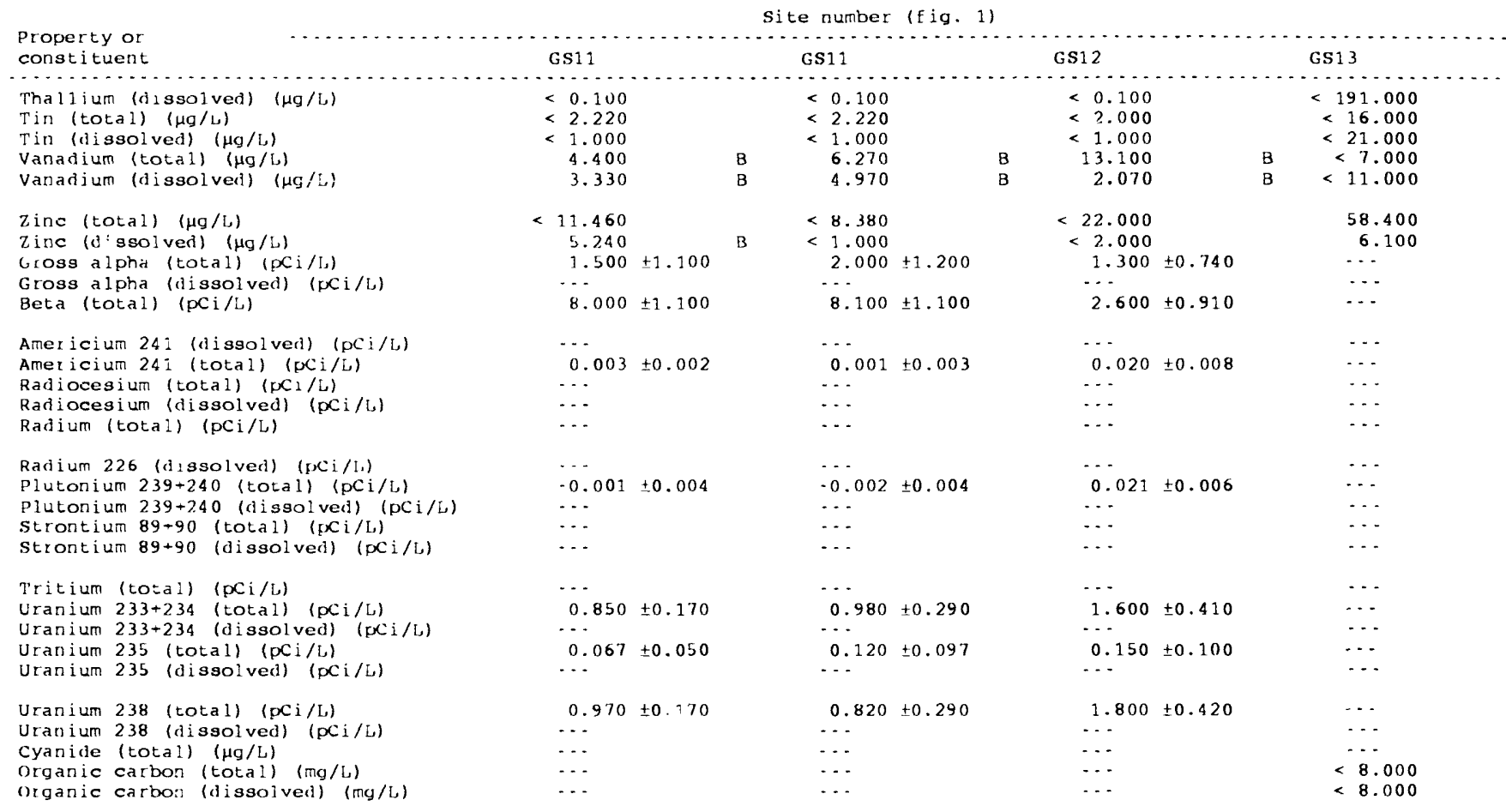


Table 41.-- Water-quality data, water year 1994--continued

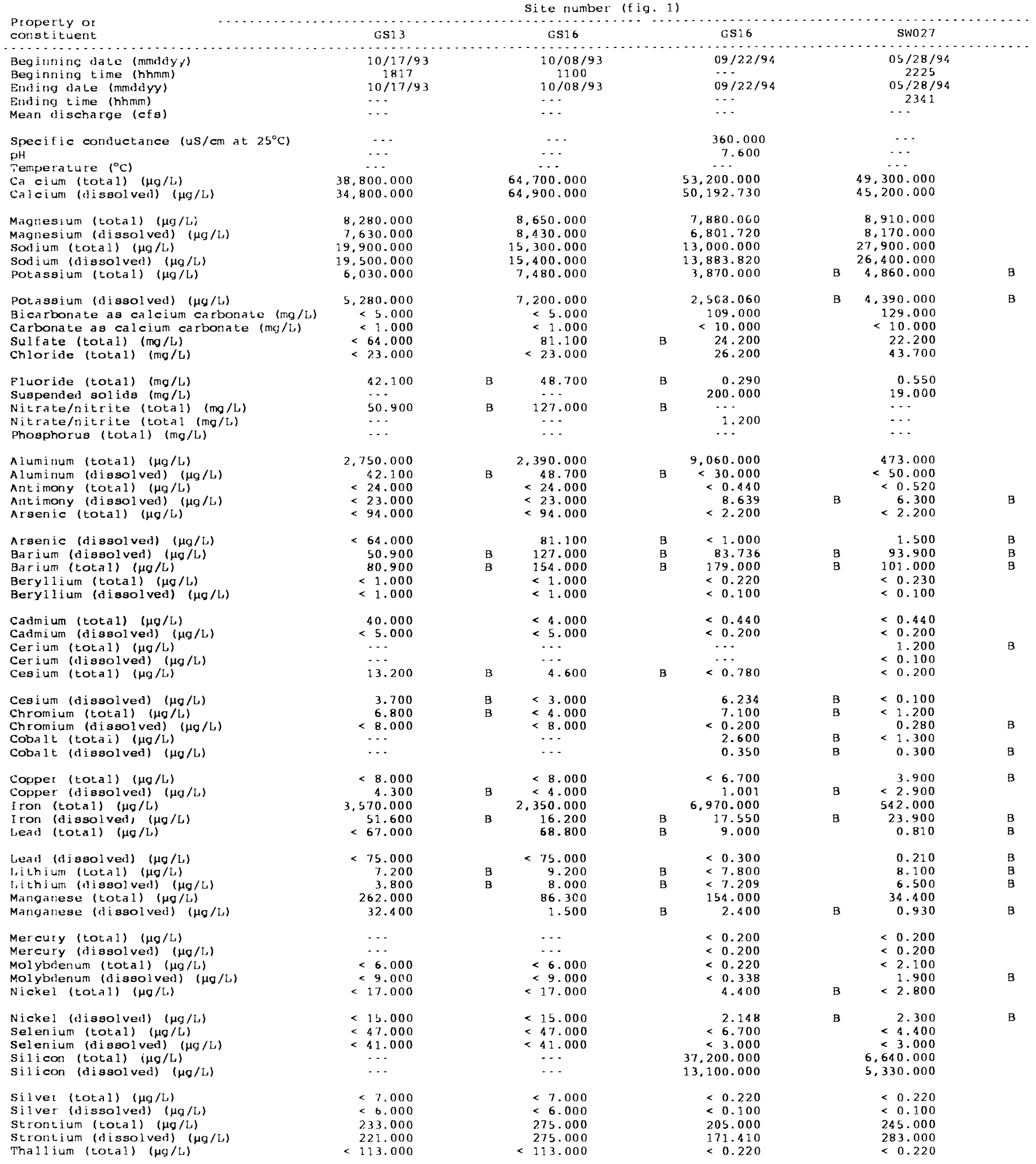


Table 41.-- Water-quality data, water year 1994--continued

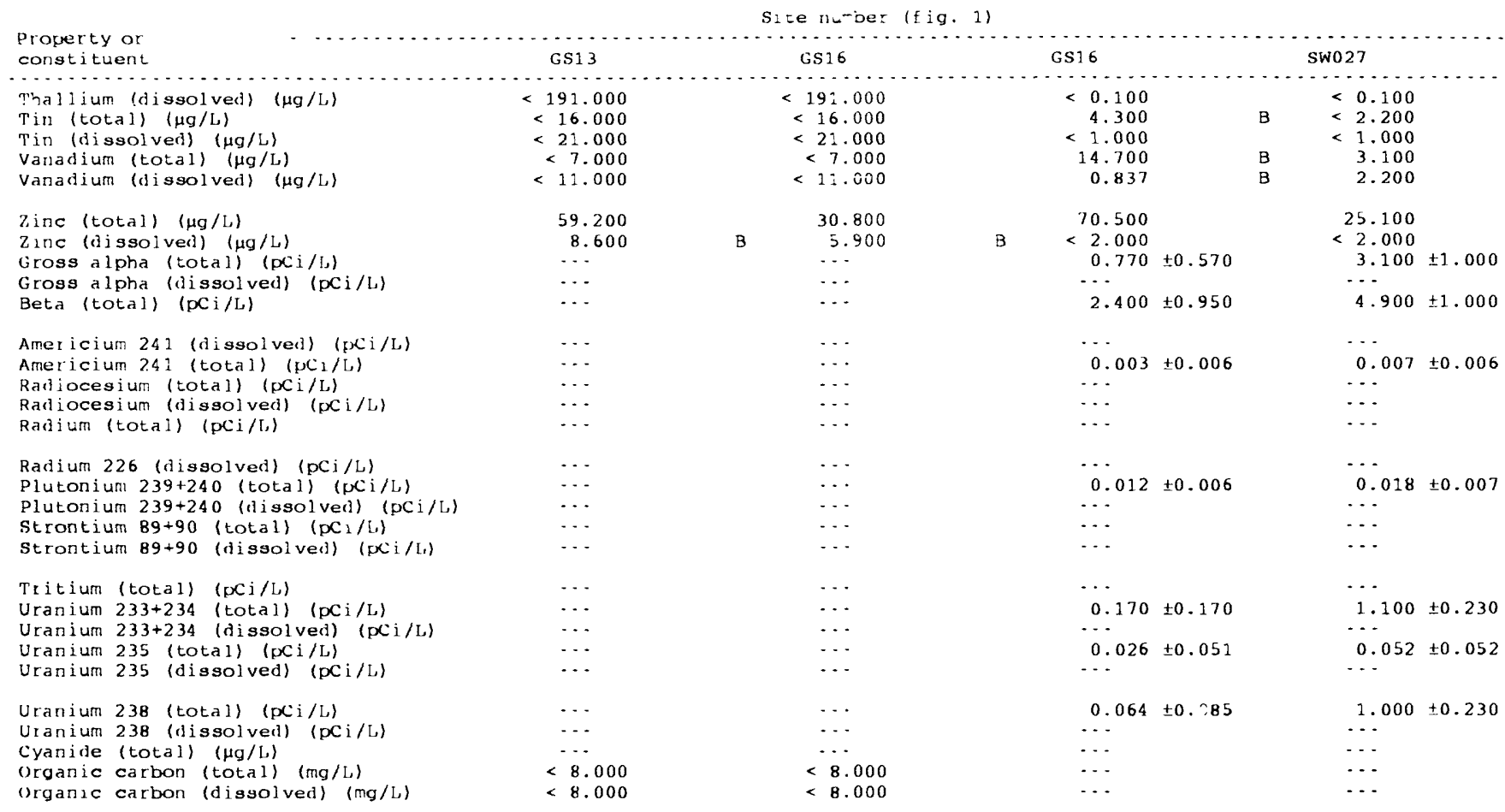


Table 41.-- Water-quality data, water year 1994--continued

\begin{tabular}{|c|c|c|c|c|c|c|c|c|c|c|c|}
\hline $\begin{array}{l}\text { Property or } \\
\text { constituent }\end{array}$ & SW027 & & & $\begin{array}{l}\text { e number } \\
\text { sw093 }\end{array}$ & ig. & & Sw093 & & & sw093 & \\
\hline $\begin{array}{l}\text { Thallium (dissolved) ( } \mu \mathrm{g} / \mathrm{L}) \\
\text { Tin (total) ( } \mathrm{g} / \mathrm{L}) \\
\text { Tin (dissolved) ( } \mu \mathrm{g} / \mathrm{L}) \\
\text { Vanadium (total) ( } \mu \mathrm{g} / \mathrm{L}) \\
\text { Vanadium (dissolved) ( } \mu \mathrm{g} / \mathrm{L})\end{array}$ & $\begin{aligned}< & 0.100 \\
< & 2.200 \\
< & 1.000 \\
& 3.800 \\
& 1.500\end{aligned}$ & & $\begin{array}{l}\mathrm{B} \\
\mathrm{B}\end{array}$ & $\begin{array}{r}<.100 \\
4.600 \\
<\quad 1.000 \\
117.000 \\
0.710\end{array}$ & & $\begin{array}{l}\mathrm{B} \\
\mathrm{B}\end{array}$ & $\begin{array}{r}<.100 \\
12.900 \\
<\quad 1.000 \\
86.900 \\
1.100\end{array}$ & & $\begin{array}{l}B \\
B\end{array}$ & $\begin{array}{l}<0.100 \\
<2.220 \\
<1.000 \\
\quad 44.200 \\
<\quad 0.200\end{array}$ & \\
\hline $\begin{array}{l}\text { Zinc (total) ( } \mu \mathrm{g} / \mathrm{L}) \\
\text { Zinc (dissolved) ( } \mu \mathrm{g} / \mathrm{L}) \\
\text { Gross a lpha (total) (pCi/L) } \\
\text { Gross a lpha (dissolved) (pCi/L) } \\
\text { Beta (total) (pCi/L) }\end{array}$ & $\begin{array}{r}26.700 \\
2.100 \\
3.200 \\
\cdots \\
4.900\end{array}$ & $\begin{array}{l} \pm 1.000 \\
\pm 0.990\end{array}$ & $\begin{array}{l}\mathrm{B} \\
\mathrm{B}\end{array}$ & $\begin{array}{r}1.230 .000 \\
4.500 \\
88.000 \\
\cdots .0 \\
97.000\end{array}$ & \pm 15.00 & $\mathrm{~B}$ & $\begin{array}{r}1.010 .000 \\
4.500 \\
56.000 \\
52.000\end{array}$ & \pm 3.500 & $B$ & $\begin{array}{r}686.270 \\
2.280 \\
110.000 \\
\quad 8.0 \\
85.000\end{array}$ & \pm 7.200 \\
\hline $\begin{array}{l}\text { Amer cium } 241 \text { (dissolved) (pci/L) } \\
\text { Americium } 241 \text { (total) (pCi/L) } \\
\text { Radiocesium (total) (pCi/L) } \\
\text { Radiocesium (dissolved) (pCi/L) } \\
\text { Radium (total) (pci/L) }\end{array}$ & $\begin{array}{l}\cdots \\
0.007 \\
\cdots \\
\cdots\end{array}$ & \pm 0.004 & & $\begin{array}{l}\cdots \\
0.640 \\
\cdots \\
\cdots\end{array}$ & \pm 0.065 & & $\begin{array}{l}\cdots \\
1.600 \\
\cdots \\
\cdots\end{array}$ & \pm 0.510 & & $\begin{array}{l}\cdots \\
1.200 \\
\cdots \\
\cdots\end{array}$ & +0.083 \\
\hline $\begin{array}{l}\text { Radium } 226 \text { (dissolved) (pCi/L) } \\
\text { Plutonium } 239+240 \text { (total) ( } \mathrm{pCi} / \mathrm{L}) \\
\text { Plutonium } 239+240 \text { (dissolved) (pCi/L) } \\
\text { Strontium } 89+90 \text { (total) (pCi/L) } \\
\text { strontium } 89+90 \text { (dissolved) ( }(\mathrm{Ni} / \mathrm{L})\end{array}$ & $\begin{array}{l}\cdots \\
0.031 \\
\cdots \\
\cdots\end{array}$ & \pm 0.009 & & $\begin{array}{l}\cdots \\
1.400 \\
\cdots \\
\cdots\end{array}$ & \pm 0.065 & & $\begin{array}{l}\cdots \\
\cdots .300 \\
\cdots \\
\cdots\end{array}$ & \pm 0.180 & & $\begin{array}{l}\cdots \\
3.500 \\
\cdots \\
\cdots\end{array}$ & \pm 0.081 \\
\hline $\begin{array}{lll}\text { Tritium } & \text { (total) } & \text { (pCi/L) } \\
\text { Uranium } 233+234 & \text { (total) (pCi/L) } \\
\text { Uranium } 233+234 & \text { (dissolved) (pCi/L) }\end{array}$ & $\cdots 100$ & \pm 0.210 & & $\begin{aligned} 110.000 \\
1.700 \\
\quad\end{aligned}$ & $\begin{array}{l} \pm 160.0 \\
\pm 0.300\end{array}$ & & $\begin{array}{r}64.000 \\
0.810 \\
-.\end{array}$ & $\begin{array}{l} \pm 150.0 \\
\pm 0.180\end{array}$ & & $\begin{array}{r}120.000 \\
1.500 \\
\cdots\end{array}$ & $\begin{array}{l} \pm 110.0 \\
\pm 0.600\end{array}$ \\
\hline $\begin{array}{ll}\text { Uranium } 235 & \text { (total) ( } \mathrm{pCi} / \mathrm{L}) \\
\text { Uranium } 235 & \text { (dissolved) (pCi/L) }\end{array}$ & $\begin{array}{l}-0.011 \\
-\cdots\end{array}$ & \pm 0.022 & & $\begin{array}{l}0.041 \\
-\cdots\end{array}$ & \pm 0.055 & & 0.320 & \pm 0.120 & & 0.130 & \pm 0.140 \\
\hline $\begin{array}{l}\text { Uranium } 238 \text { (total) (pCi/L) } \\
\text { Uranium } 238 \text { (dissolved) ( } \mathrm{pCi} / \mathrm{L}) \\
\text { Cyanide (total) ( } \mu \mathrm{g} / \mathrm{L}) \\
\text { Organic carbon (total) (mg/L) } \\
\text { Organic carbon (dissolved) (mg/L) }\end{array}$ & $\begin{array}{l}1.100 \\
\cdots \\
\cdots \\
\cdots\end{array}$ & \pm 0.210 & & $\begin{array}{l}1.700 \\
\cdots \\
\cdots \\
\cdots \\
\cdots\end{array}$ & \pm 0.300 & & $\begin{array}{l}1.000 \\
\cdots \\
\cdots \\
\cdots\end{array}$ & \pm 0.200 & & $\begin{array}{l}1.500 \\
\cdots \\
\cdots \\
\cdots\end{array}$ & \pm 0.600 \\
\hline
\end{tabular}


Table 41.-- Water-quality data, water year 1994--continued

\begin{tabular}{|c|c|c|c|c|c|c|c|c|}
\hline $\begin{array}{l}\text { Property or } \\
\text { constituent }\end{array}$ & sw093 & & $\begin{array}{c}\text { Site number } \\
\text { sw093 }\end{array}$ & & SW1 34 & & SW998 & \\
\hline $\begin{array}{l}\text { Berguning date (mmddyy) } \\
\text { Beginning time (hhmm) } \\
\text { Ending date (mmddyy) } \\
\text { Ending time (hhmm) } \\
\text { Mean discharge (cfs) }\end{array}$ & $\begin{array}{c}06 / 22 / 94 \\
1804 \\
06 / 22 / 94 \\
1818 \\
0.550\end{array}$ & & $\begin{array}{c}08 / 10 / 94 \\
2143 \\
08 / 10 / 94 \\
2156 \\
2.730\end{array}$ & & $\begin{array}{c}08 / 11 / 94 \\
1144 \\
08 / 11 / 94 \\
1158 \\
0.830\end{array}$ & & $\begin{array}{c}05 / 28 / 94 \\
1335 \\
05 / 28 / 94 \\
1350 \\
\end{array}$ & \\
\hline $\begin{array}{l}\text { Speciflc conductance }\left(\mathrm{uS} / \mathrm{cm} \text { at } 25^{\circ} \mathrm{C}\right) \\
\text { pht } \\
\left.\text { Temperature }{ }^{\circ} \mathrm{C}\right) \\
\text { Calcium (total) ( } \mu \mathrm{g} / \mathrm{L}) \\
\text { Calcium (dissolved) ( } \mu \mathrm{g} / \mathrm{L})\end{array}$ & $\begin{array}{r}340.000 \\
1.200 \\
\quad . . \\
40.437 .102 \\
36.721 .500\end{array}$ & & $\begin{array}{r}130.000 \\
7.900 \\
20.500 \\
65,435.020 \\
12.907 .600\end{array}$ & & $\begin{array}{r}230.000 \\
7.400 \\
24.600 \\
25,093.400 \\
21,741.760\end{array}$ & & $\begin{array}{c}\cdots \\
14,400.000 \\
10,400.000\end{array}$ & \\
\hline $\begin{array}{l}\text { Magnesium (total) ( } \mu \mathrm{g} / \mathrm{L}) \\
\text { Magnesium (dissolved) ( } \mu \mathrm{g} / \mathrm{L}) \\
\text { Sodium (total) ( } \mu \mathrm{g} / \mathrm{L}) \\
\text { Sodium (dissolved) ( } \mu \mathrm{g} / \mathrm{L}) \\
\text { Potassium (total) ( } \mathrm{gg} / \mathrm{L})\end{array}$ & $\begin{array}{r}8,897.600 \\
7.534 .300 \\
20.847 .699 \\
20,926.600 \\
4.283 .400\end{array}$ & $B$ & $\begin{array}{r}9,606.680 \\
1,837.930 \\
6,799.200 \\
5,565.980 \\
10,619.730\end{array}$ & $\mathbf{B}$ & $\begin{array}{r}5,915.340 \\
4.486 .140 \\
15,372.420 \\
14.039 .840 \\
4.598 .170\end{array}$ & $B$ & $\begin{array}{r}4,860.000 \\
1,800.000 \\
16,000.000 \\
13,300.000 \\
4,130.000\end{array}$ & $\begin{array}{l}3 \\
3\end{array}$ \\
\hline $\begin{array}{l}\text { Potassium (dissolved) ( } \mu \mathrm{g} / \mathrm{L}) \\
\text { Bicarbonate as calcium carbonate (mg/L) } \\
\text { Carbonate as calcium carbonate (mg/L) } \\
\text { Sulfate (total) (mg/L) } \\
\text { Chloride (total) (mg/L) }\end{array}$ & $\begin{array}{r}3.159 .500 \\
115.000 \\
<\quad 10.000 \\
<5.000 \\
27.300\end{array}$ & $B$ & $\begin{array}{r}2.494 .940 \\
78.900 \\
<\quad 10.000 \\
9.600 \\
8.000\end{array}$ & $B$ & $\begin{array}{r}1,993.820 \\
51.200 \\
<\quad 10.000 \\
36.200 \\
10.700\end{array}$ & B & $\begin{array}{r}2,110.000 \\
25.400 \\
<10.000 \\
22.000 \\
14.300\end{array}$ & 3 \\
\hline $\begin{array}{l}\text { Fluoride (total) (mg/L) } \\
\text { Suspended solids (mg/L) } \\
\text { Nitrate/nitrite (total) (mg/L) } \\
\text { Nitrate/nitrite (total (mg/L) } \\
\text { Phosphorus (total) (mg/L) }\end{array}$ & $\begin{array}{l}0.270 \\
110.000 \\
0.720 \\
\cdots\end{array}$ & & $\begin{array}{l}0.160 \\
500.000 \\
0.680 \\
\cdots \\
\cdots\end{array}$ & & $\begin{array}{l}0.640 \\
140.000 \\
1.200 \\
\cdots\end{array}$ & & $\begin{array}{l}0.140 \\
300.000 \\
\cdots \\
\cdots\end{array}$ & \\
\hline 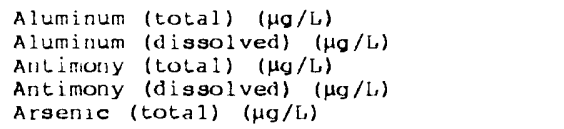 & $\begin{array}{r}6,176.500 \\
<50.000 \\
<\quad 0.630 \\
8.670 \\
2.680\end{array}$ & $\begin{array}{l}B \\
B\end{array}$ & $\begin{aligned} & 37.854 .699 \\
&< 30.000 \\
&< 4.600 \\
&< 1.600 \\
&< 1.000\end{aligned}$ & & $\begin{array}{r}12,359.970 \\
35.840 \\
6.300 \\
<0.440 \\
<1.000\end{array}$ & $\begin{array}{l}\mathrm{B} \\
\mathrm{B}\end{array}$ & $\begin{array}{r}5,760.000 \\
<\quad 50.000 \\
<\quad 1.100 \\
\quad 16.800 \\
<5.600\end{array}$ & $B$ \\
\hline 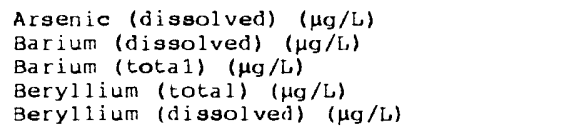 & $\begin{array}{r}<2.190 \\
59.580 \\
113.990 \\
<\quad 0.220 \\
<0.100\end{array}$ & $\begin{array}{l}\text { B } \\
\text { B }\end{array}$ & $\begin{array}{r}11.600 \\
426.000 \\
22.100 \\
<\quad 0.140 \\
<\quad 1.400\end{array}$ & $\begin{array}{l}\mathrm{B} \\
\mathrm{B} \\
\mathrm{B}\end{array}$ & $\begin{array}{r}<2.200 \\
132.000 \\
54.400 \\
<\quad 0.190 \\
<0.760\end{array}$ & $\begin{array}{l}\mathrm{B} \\
\mathrm{B}\end{array}$ & $\begin{array}{r}<1.000 \\
22.100 \\
125.000 \\
<0.600 \\
<0.100\end{array}$ & $\begin{array}{l}B \\
B\end{array}$ \\
\hline 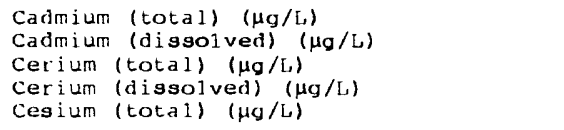 & $\begin{array}{r}<.440 \\
0.200 \\
8.540 \\
<0.100 \\
0.660\end{array}$ & $\begin{array}{l}B \\
B\end{array}$ & $\begin{array}{l}<0.200 \\
1.700 \\
\cdots \\
\cdots \\
<0.100\end{array}$ & B & $\begin{array}{l}<0.200 \\
<0.440 \\
\quad \cdots \\
< \\
<0.120\end{array}$ & & $\begin{array}{r}<1.900 \\
<0.200 \\
27.700 \\
0.140 \\
1.500\end{array}$ & $\begin{array}{l}\mathrm{B} \\
\mathrm{B} \\
\mathrm{B}\end{array}$ \\
\hline $\begin{array}{l}\text { Cesium (dissolved) }(\mu \mathrm{g} / \mathrm{L}) \\
\text { Chromium (total) }(\mu \mathrm{g} / \mathrm{L}) \\
\text { Chromium (disgolved) }(\mu \mathrm{g} / \mathrm{L}) \\
\text { Cobalt (total) ( } \mu \mathrm{g} / \mathrm{L}) \\
\text { Cobalt (dissolved) }(\mu \mathrm{g} / \mathrm{L})\end{array}$ & $\begin{array}{l}0.100 \\
9.920 \\
0.570 \\
1.750 \\
0.150\end{array}$ & $\begin{array}{l}\mathrm{B} \\
\mathrm{B} \\
\mathrm{B} \\
\mathrm{B}\end{array}$ & $\begin{array}{r}5.500 \\
0.670 \\
49.900 \\
0.360 \\
13.700\end{array}$ & $B$ & $\begin{array}{r}2.800 \\
0.510 \\
15.400 \\
0.460 \\
<\quad 1.800\end{array}$ & $\begin{array}{l}\mathrm{B} \\
\mathrm{B} \\
\mathrm{B}\end{array}$ & $\begin{array}{r}<0.100 \\
<\quad 28.300 \\
1.400 \\
<\quad 3.400 \\
0.120\end{array}$ & $B$ \\
\hline $\begin{array}{l}\text { Copper (tota } 1)(\mu \mathrm{g} / \mathrm{L}) \\
\text { Coppet (dissolved) }(\mu \mathrm{g} / \mathrm{L}) \\
\text { I on (total) }(\mu \mathrm{g} / \mathrm{L}) \\
\text { I ron (dissolved) }(\mu \mathrm{g} / \mathrm{L}) \\
\text { Lead }(\text { total) }(\mu \mathrm{g} / \mathrm{L})\end{array}$ & $\begin{array}{r}241.560 \\
4.420 \\
5,270.300 \\
<\quad 20.000 \\
52.730\end{array}$ & B & $\begin{array}{r}9.800 \\
111.000 \\
41.585 .031 \\
51.490 \\
0.240\end{array}$ & $\begin{array}{l}\text { B } \\
\text { B }\end{array}$ & $\begin{array}{r}2.400 \\
<9.200 \\
5.165 .430 \\
10.290 \\
0.120\end{array}$ & $\begin{array}{l}\mathrm{B} \\
\mathrm{B}\end{array}$ & $\begin{array}{r}12.500 \\
5.700 \\
6,940.000 \\
33.900 \\
91.600\end{array}$ & $\begin{array}{l}3 \\
B\end{array}$ \\
\hline 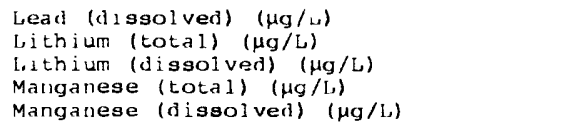 & $\begin{array}{r}<.100 \\
6.960 \\
3.630 \\
317.200 \\
45.260\end{array}$ & $\begin{array}{l}\mathrm{B} \\
\mathrm{B}\end{array}$ & $\begin{array}{r}111.000 \\
1.600 \\
23.500 \\
63.800 \\
2.130 .000\end{array}$ & B & $\begin{array}{r}8.800 \\
2.600 \\
4.100 \\
12.900 \\
39.700\end{array}$ & $\begin{array}{l}\mathrm{B} \\
\mathrm{B} \\
\mathrm{B}\end{array}$ & $\begin{array}{r}0.390 \\
<\quad 7.200 \\
<\quad 1.000 \\
300.000 \\
8.100\end{array}$ & B \\
\hline $\begin{array}{l}\text { Merculy (total) }(\mu \mathrm{g} / \mathrm{L}) \\
\text { Mercury (dissolved) ( } \mu \mathrm{g} / \mathrm{L}) \\
\text { Molybdenum (total) ( } \mathrm{g} / \mathrm{L}) \\
\text { Molybdenum (dissolved) ( } \mathrm{gg} / \mathrm{L}) \\
\text { Nickel (total) }(\mu \mathrm{g} / \mathrm{L})\end{array}$ & $\begin{array}{r}<0.200 \\
<0.200 \\
<\quad 1.200 \\
1.190 \\
6.630\end{array}$ & $\begin{array}{l}\mathrm{B} \\
\mathrm{B}\end{array}$ & $\begin{array}{r}<0.200 \\
<0.200 \\
0.510 \\
<\quad 1.800 \\
1.600\end{array}$ & B & $\begin{aligned}< & 0.200 \\
< & 0.200 \\
& 2.600 \\
< & 2.400 \\
& 1.600\end{aligned}$ & B & $\begin{array}{l}<0.200 \\
<0.200 \\
<1.400 \\
<0.260 \\
<2.900\end{array}$ & \\
\hline $\begin{array}{l}\text { Nickel (dissolved) ( } \mu \mathrm{g} / \mathrm{L}) \\
\text { Selenium (total) ( } \mathrm{gg} / \mathrm{L}) \\
\text { Selentum (dissolved) ( } \mu \mathrm{g} / \mathrm{L}) \\
\text { Silicon (total) }(\mu \mathrm{g} / \mathrm{L}) \\
\text { Silicon (dissolved) ( } \mu \mathrm{g} / L)\end{array}$ & $\begin{array}{r}<1.120 \\
<2.200 \\
<1.000 \\
15,998.700 \\
3.648 .100\end{array}$ & & $\begin{array}{r}29.100 \\
<3.000 \\
<6.700 \\
73,325.227 \\
1,017.690\end{array}$ & B & $\begin{array}{r}7.300 \\
<3.000 \\
<6.700 \\
26,702.650 \\
5.644 .470\end{array}$ & $B$ & $\begin{array}{r}1.200 \\
<\quad 11.100 \\
<\quad 3.000 \\
11,700.000 \\
739.000\end{array}$ & B \\
\hline 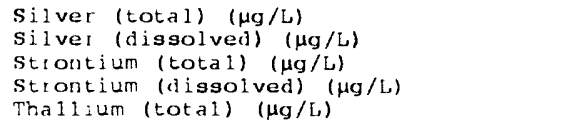 & $\begin{array}{r}<0.220 \\
<0.100 \\
250.090 \\
<205.000 \\
<0.220\end{array}$ & & $\begin{array}{r}<0.100 \\
<0.240 \\
64.500 \\
189.000 \\
<0.100\end{array}$ & & $\begin{array}{l}<0.100 \\
<0.220 \\
120.000 \\
147.000 \\
<0.100\end{array}$ & & $\begin{array}{r}<0.940 \\
<0.100 \\
93.000 \\
56.800 \\
<\quad 0.600\end{array}$ & \\
\hline
\end{tabular}


Table 41.-- Water-quality data, water year 1994--continued

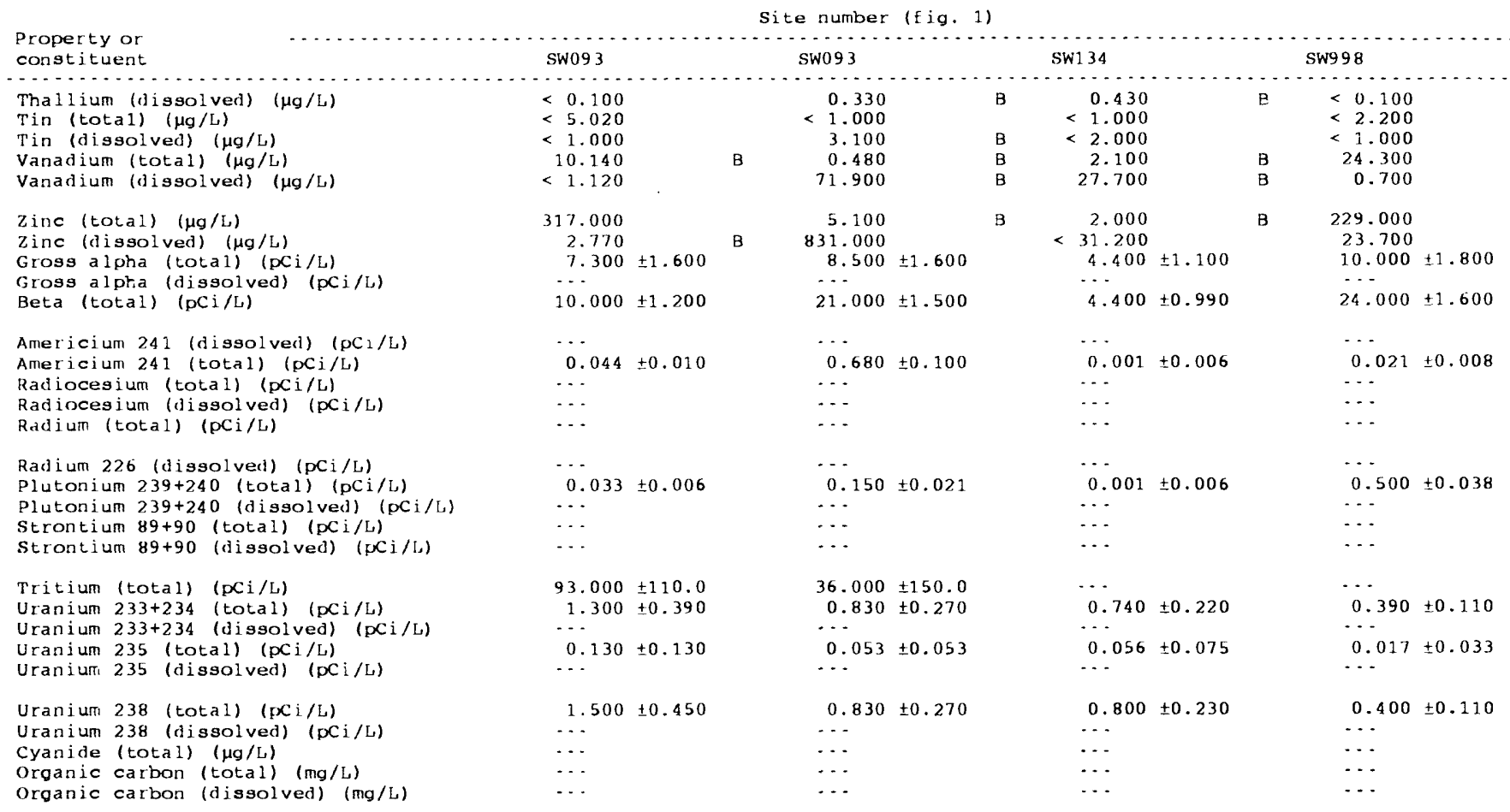


Table 41.-- Water-quality data, water year 1994--continued

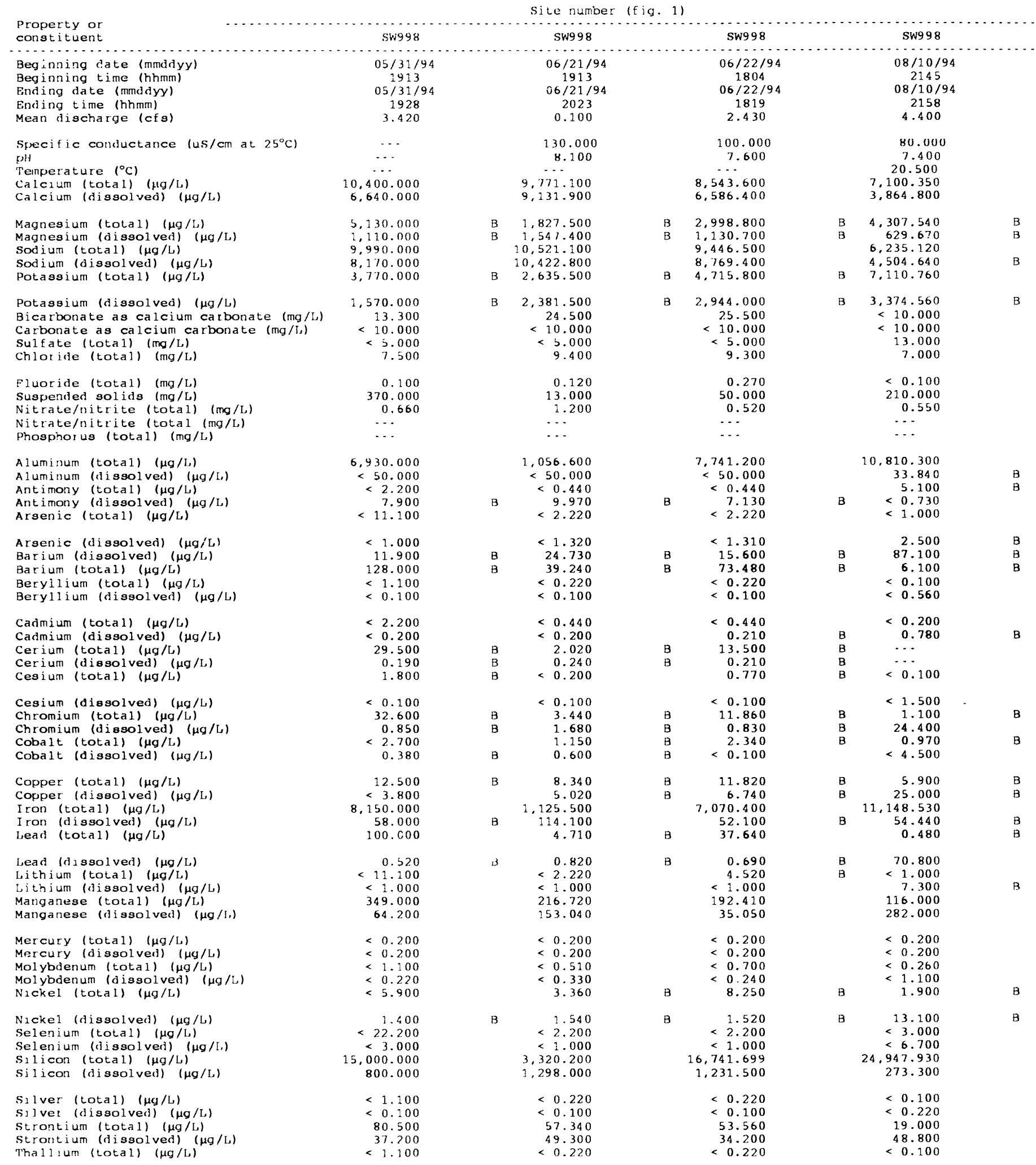


Table 41.-- Water-quality data, water year 1994--continued

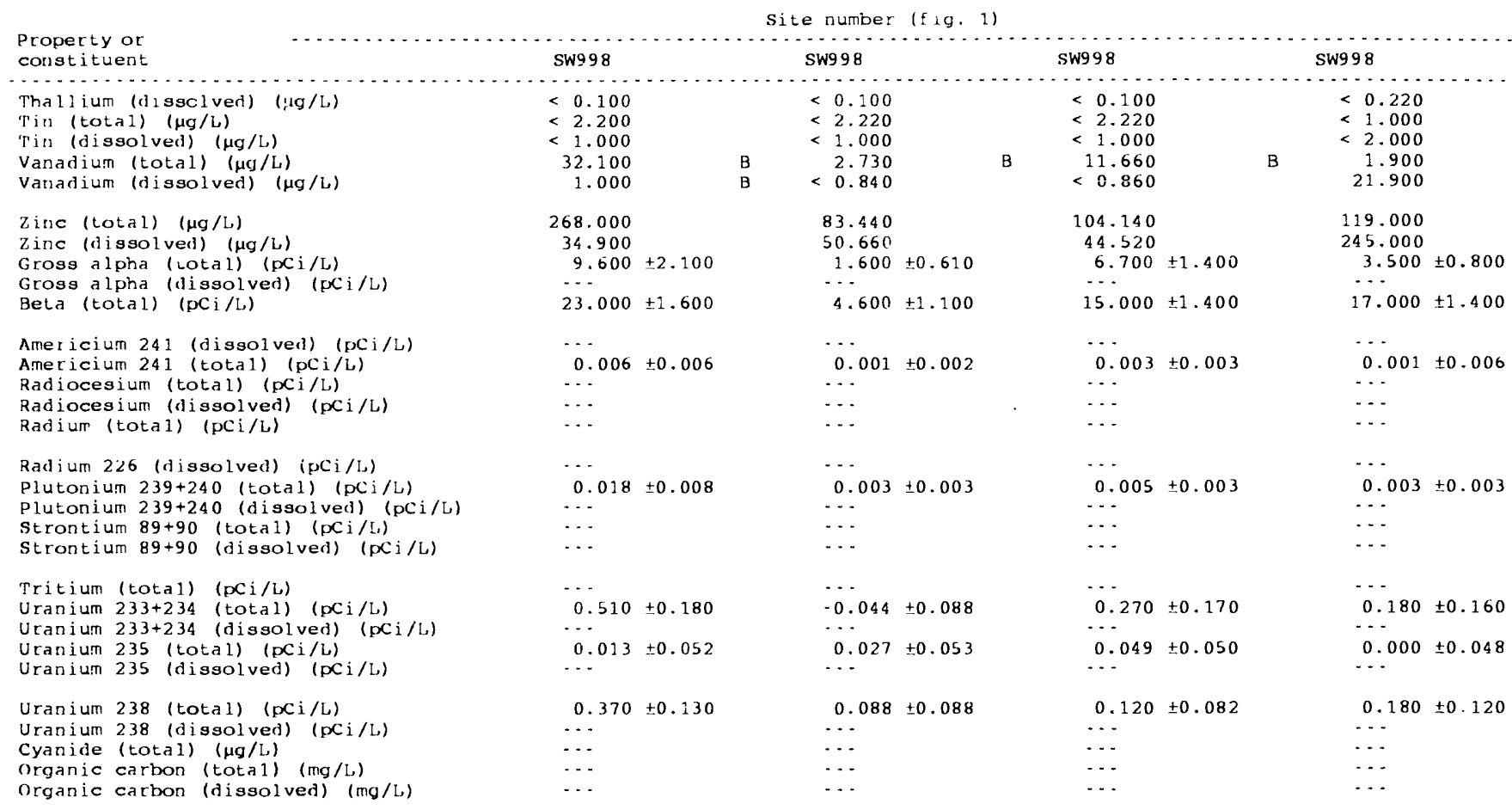




\section{Table 41.-- Water-quality data, water year 1994--continued}

Site number (fig. 1 )

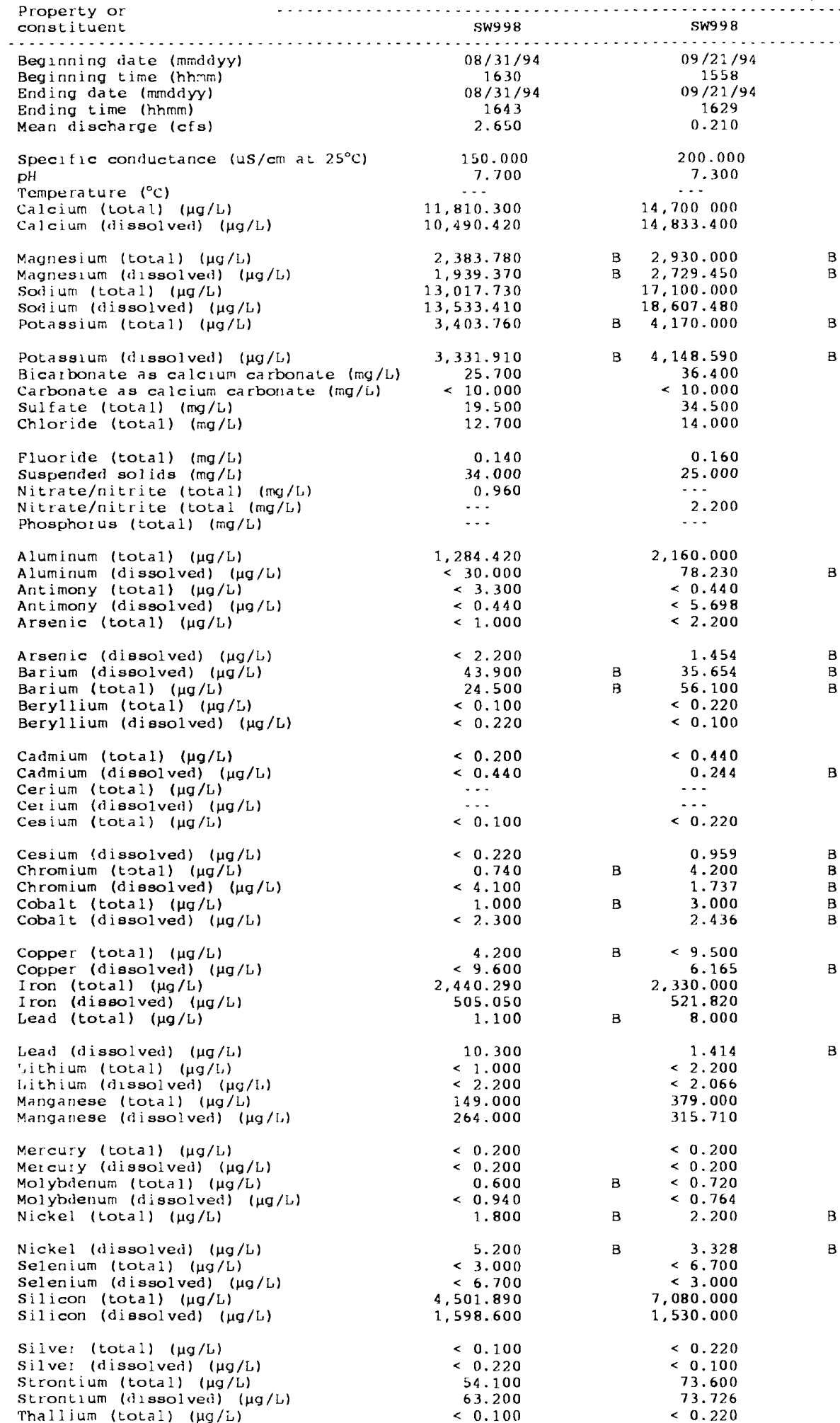


Table 41.-- Water-quality data, water year 1994--continued

Site number (Eig. 1)

\begin{tabular}{|c|c|c|c|c|c|}
\hline $\begin{array}{l}\text { Property or } \\
\text { const ituent }\end{array}$ & SW998 & & & SW998 & \\
\hline $\begin{array}{l}\text { Thallium (dissolve(?) ( } \mu \mathrm{g} / \mathrm{L}) \\
\text { Tin (tota } 1)(\mu \mathrm{g} / \mathrm{L}) \\
\text { Tin (dissolved) ( } \mu \mathrm{g} / \mathrm{L}) \\
\text { Vanadium (total) }(\mu \mathrm{g} / \mathrm{L}) \\
\text { Vanadium (dissolved) }(\mu \mathrm{g} / \mathrm{L})\end{array}$ & $\begin{array}{r}0.240 \\
<\quad 1.000 \\
<\quad 2.000 \\
\\
0.680 \\
<\quad 4.000\end{array}$ & & B & $\begin{aligned}< & 0.100 \\
< & 2.000 \\
< & 1.000 \\
< & 5.500 \\
& 1.004\end{aligned}$ & \\
\hline $\begin{array}{l}\text { Zinc (total) }(\mu \mathrm{g} / \mathrm{L}) \\
\text { Zinc (dissolved) ( } \mu \mathrm{g} / \mathrm{L}) \\
\text { Gross a lpha (total) (pCi/L) } \\
\text { Gross aipha (dissolved) (pci/L) } \\
\text { Beta (total) (pCi/L) }\end{array}$ & $\begin{array}{r}64.100 \\
93.200 \\
2.300 \\
\quad 6 . \\
6.800\end{array}$ & \pm 1.100 & & $\begin{array}{r}153.000 \\
119.500 \\
5.200 \\
12.000\end{array}$ & \pm 1.200 \\
\hline $\begin{array}{l}\text { Americium } 241 \text { (dissolved) ( } \mathrm{pi} / \mathrm{L}) \\
\text { Americium } 241 \text { (total) (pCi/L) } \\
\text { Radiocesium (total) (pci/L) } \\
\text { Radiocesium (dissolved) (pci } / \mathrm{L}) \\
\text { Radium (total) (pCi/L) }\end{array}$ & $\begin{array}{l}\cdots \\
0.002 \\
\cdots \\
\cdots\end{array}$ & \pm 0.003 & & $\begin{array}{l}\cdots \\
0.004 \\
\cdots \\
\cdots \\
0.540\end{array}$ & \pm 0.006 \\
\hline $\begin{array}{l}\text { Radium } 226 \text { (dissolved) (pCi/L) } \\
\text { Plutonium } 239+240 \text { (total) (pCi/L) } \\
\text { plutonium } 239+240 \text { (dissoived) (pCi/L) } \\
\text { Strontium } 89+90 \text { (total) (pCi } / \mathrm{L}) \\
\text { Strontium } 89+90 \text { (dissolved) (pCi/L) }\end{array}$ & $\begin{array}{l}\cdots \\
0.003 \\
\cdots \\
\cdots\end{array}$ & \pm 0.003 & & $\begin{array}{l}\cdots \\
0.008 \\
\cdots \\
\cdots\end{array}$ & \pm 0.005 \\
\hline $\begin{array}{lll}\text { Tritium } & \text { (total) } & \text { (pci } / L) \\
\text { Uranium } & 233+234 \text { (total) (pCi } / L) \\
\text { Uranium } & 233+234 \text { (dissolved) (pCi } / L) \\
\text { Uranium } 235 \text { (total) (pci } / L) \\
\text { Uranium } 235 \text { (dissolved) (pci } / L)\end{array}$ & $\begin{array}{l}\ldots \\
0.032 \\
-0.039 \\
\ldots .0\end{array}$ & $\begin{array}{l} \pm 0.130 \\
\pm 0.077\end{array}$ & & $\begin{array}{l}\ldots \\
0.068 \\
0.000 \\
\ldots\end{array}$ & $\begin{array}{l} \pm 0.092 \\
\pm 0.055\end{array}$ \\
\hline $\begin{array}{l}\text { Uranium } 238 \text { (Lotal) (pci/L) } \\
\text { Uranium } 238 \text { (dissolved) (pCi/L) } \\
\text { Cyanile (total) ( } \mu \mathrm{g} / \mathrm{L}) \\
\text { Organic carbon (total) (mg/L) } \\
\text { Organic carbon (dissolved) (mg/L) }\end{array}$ & $\begin{array}{l}0.190 \\
\cdots \\
\cdots \\
\cdots\end{array}$ & \pm 0.130 & & $\begin{array}{l}0.068 \\
\cdots \\
\cdots \\
\cdots\end{array}$ & \pm 0.092 \\
\hline
\end{tabular}


Table 42.-- Water-quality data, water year 1995--continued

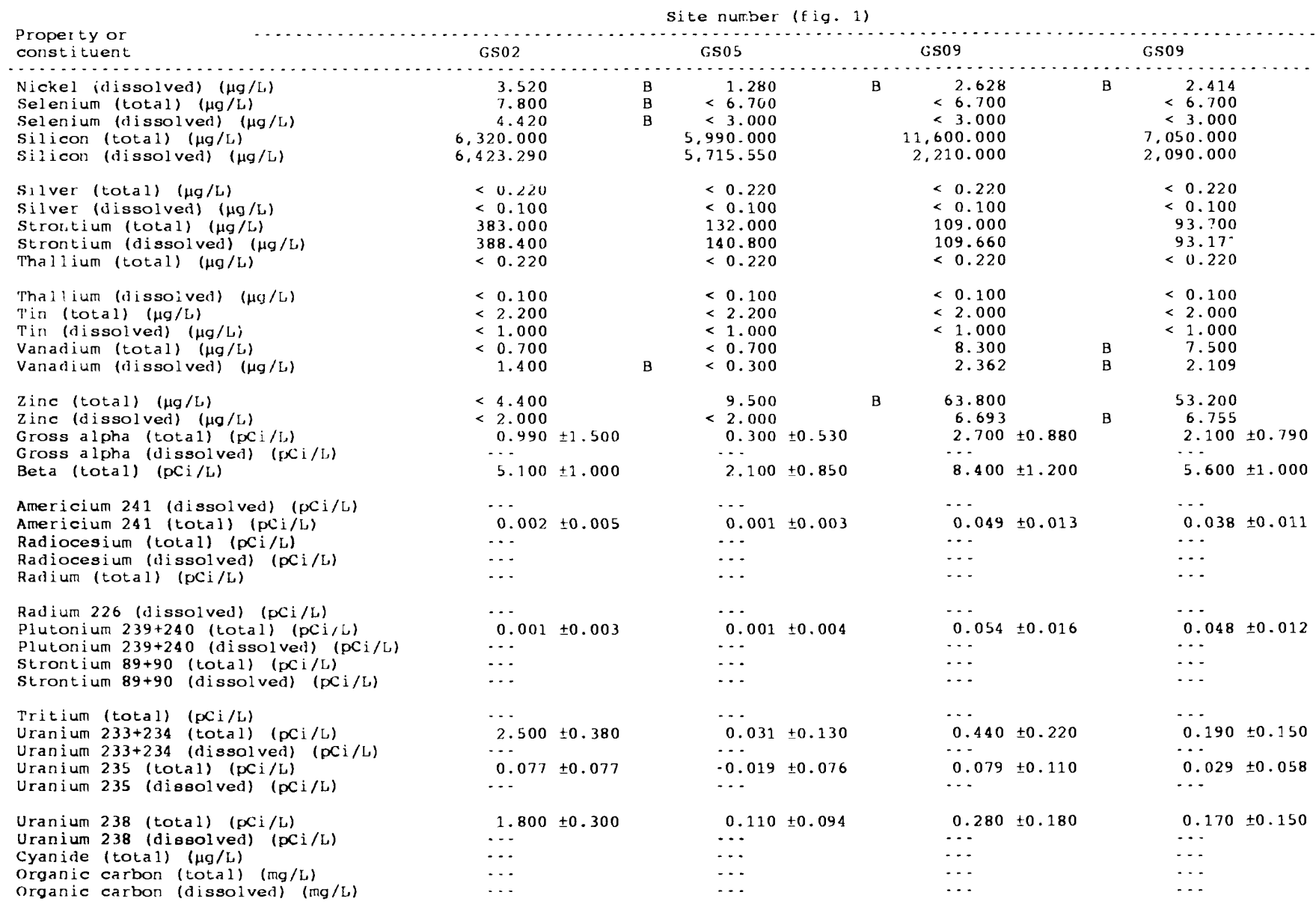


Table 42.-- Water-quality data, water year 1995--continued

\begin{tabular}{|c|c|c|c|c|c|c|c|c|}
\hline $\begin{array}{l}\text { Property or } \\
\text { constituent }\end{array}$ & Gsog & & $\begin{array}{c}\text { Site number } \\
\text { Gsio }\end{array}$ & & GS10 & & GS10 & \\
\hline $\begin{array}{l}\text { Beginning late (mmddyy) } \\
\text { Beginning time (hhmm) } \\
\text { Ending late (mmddyy) } \\
\text { Ending time (hhmm) } \\
\text { Mean discharge (cfs) }\end{array}$ & $\begin{aligned} & 1: / 98 / 94 \\
&: 649 \\
& 1:=08 / 94 \\
& 1759 \\
& 2.310\end{aligned}$ & & $\begin{array}{c}10 / 17 / 9.4 \\
0625 \\
10 / 17 / 94 \\
0638 \\
0.6: 0\end{array}$ & & $\begin{array}{l}10 / 17 / 94 \\
0951 \\
10 / 17 / 94 \\
1005 \\
2.120\end{array}$ & & $\begin{array}{l}10 / 28 / 94 \\
1330 \\
10 / 28 / 94 \\
0.120\end{array}$ & \\
\hline $\begin{array}{l}\left.\text { Specific conductance (uS/cm at } 25^{\circ} \mathrm{C}\right) \\
\text { pH } \\
\text { Temperature }\left({ }^{\circ} \mathrm{C}\right) \\
\text { Calcium (total) }(\mu \mathrm{g} / \mathrm{L}) \\
\text { Calcium (dissolved) ( } \mu \mathrm{g} / \mathrm{L})\end{array}$ & $\begin{array}{r}390.000 \\
7.400 \\
\vdots 0.000 \\
29.300 .000 \\
27.856 .740\end{array}$ & & $\begin{array}{c}530.000 \\
\cdots \\
50.900 .000 \\
53.429 .949\end{array}$ & & $\begin{array}{r}120.000 \\
7.600 \\
\cdots \\
12,700.000 \\
11.997 .540\end{array}$ & & $\begin{array}{c}10.000 \\
4.900 \\
\quad \therefore \\
532.000 \\
428.480\end{array}$ & \\
\hline $\begin{array}{l}\text { Magnesium (total) ( } \mu \mathrm{g} / \mathrm{L}) \\
\left.\text { Magnesium (dissolved) ( } \mu \mathrm{g} / \mathrm{L}_{1}\right) \\
\text { Sodium (total) ( } \mu \mathrm{g} / \mathrm{L}) \\
\left.\text { Sodium (dissolved) ( } \mathrm{gg}_{\mathrm{L}}\right) \\
\text { Potassium (total) ( } \mu \mathrm{g} / \mathrm{L})\end{array}$ & $\begin{array}{r}5.610 .000 \\
4,952.590 \\
36,500.000 \\
39.443 .801 \\
7.080 .000\end{array}$ & B & $\begin{array}{r}14,000.000 \\
15,049.320 \\
37,400.000 \\
45,199.371 \\
3,370.000\end{array}$ & B & $\begin{array}{l}2,770.000 \\
2,172.430 \\
7,100.000 \\
7,681.860 \\
3,370.000\end{array}$ & $\begin{array}{l}\mathrm{B} \\
\mathrm{B}\end{array}$ & $\begin{array}{l}<222.200 \\
<200.000 \\
<333.300 \\
<300.000 \\
<333.300\end{array}$ & \\
\hline $\begin{array}{l}\text { Polassium (dissolvei) }(\mu \mathrm{g} / \mathrm{L}) \\
\text { Bicarbonate as calcium carbonate (mg/L) } \\
\text { Carbonate as calcium carbonate (mg/L) } \\
\text { Sulfate (total) (mg/L) } \\
\text { Chloride (total) (mg/L) }\end{array}$ & $\begin{array}{r}6.843 .460 \\
58.100 \\
<\quad 10.000 \\
25.600 \\
49.100\end{array}$ & & $\begin{array}{r}3.9: 8.390 \\
161.000 \\
<\quad 10.000 \\
27.600 \\
50.300\end{array}$ & B & $\begin{array}{r}2.702 .700 \\
24.200 \\
<\quad 10.000 \\
10.300 \\
8.100\end{array}$ & $B$ & $\begin{array}{l}<300.000 \\
<\quad 10.000 \\
<10.000 \\
<5.000 \\
<2.000\end{array}$ & \\
\hline $\begin{array}{l}\text { Fluoride (total) }(\mathrm{mg} / \mathrm{L}) \\
\text { Suspended solids }(\mathrm{mg} / \mathrm{L}) \\
\text { Nitrate/nitrite (total) (mg/L) } \\
\text { Nitrate/nitrite (total (mg/L) } \\
\text { Phosphorus (total) (mg/L) }\end{array}$ & $\begin{array}{l}0.340 \\
96.000 \\
\cdots \\
2.400 \\
\cdots\end{array}$ & & $\begin{array}{l}0.610 \\
34.000 \\
\cdots \\
2.200\end{array}$ & & $\begin{array}{l}0.180 \\
59.000 \\
\ldots \\
0.630 \\
\cdots\end{array}$ & & 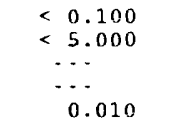 & B \\
\hline 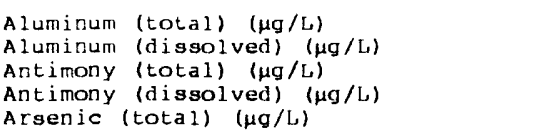 & $\begin{array}{r}4.270 .000 \\
<\quad 30.000 \\
7.800 \\
12.690 \\
3.700\end{array}$ & $\begin{array}{l}\mathrm{B} \\
\mathrm{B} \\
\mathrm{B}\end{array}$ & $\begin{array}{r}2.340 .000 \\
<\quad 30.000 \\
78.300 \\
85.217 \\
4.400\end{array}$ & B & $\begin{array}{r}5,320.000 \\
45.680 \\
39.700 \\
44.070 \\
3.000\end{array}$ & $\begin{array}{l}\mathrm{B} \\
\mathrm{B} \\
\mathrm{B} \\
\mathrm{B}\end{array}$ & $\begin{array}{l}<53.800 \\
<\quad 30.000 \\
<\quad 0.440 \\
4.040 \\
<2.200\end{array}$ & B \\
\hline $\begin{array}{l}\text { Arsenic (dissolved) ( } \mu \mathrm{g} / \mathrm{L}) \\
\text { Barium (dissolved) } \\
\text { Barium (total) ( } \mu \mathrm{gg} / \mathrm{L}) \\
\text { Berylium (total) }(\mu \mathrm{g} / \mathrm{L}) \\
\text { Beryllium (dissolved) ( } \mathrm{gg} / \mathrm{L})\end{array}$ & $\begin{array}{r}1.830 \\
26.390 \\
63.500 \\
<\quad 0.220 \\
<\quad 0.100\end{array}$ & $\begin{array}{l}\mathrm{B} \\
\mathrm{B} \\
\mathrm{B}\end{array}$ & $\begin{array}{r}3.006 \\
76.689 \\
104.000 \\
<\quad 0.220 \\
<\quad 0.100\end{array}$ & $\begin{array}{l}\mathrm{B} \\
\mathrm{B} \\
\mathrm{B}\end{array}$ & $\begin{array}{r}1.913 \\
19.749 \\
54.700 \\
<\quad 0.220 \\
<\quad 0.100\end{array}$ & $\begin{array}{l}\mathrm{B} \\
\mathrm{B} \\
\mathrm{B}\end{array}$ & $\begin{array}{l}<1.000 \\
<0.200 \\
1.900 \\
<0.220 \\
<0.100\end{array}$ & B \\
\hline $\begin{array}{l}\text { Cadmium (total) }(\mu \mathrm{g} / \mathrm{L}) \\
\text { Cadmium (dissolved) }(\mu \mathrm{g} / \mathrm{L}) \\
\text { Cerium (total) ( } \mu \mathrm{g} / \mathrm{L}) \\
\text { Cerium (dissolved) ( } \mu \mathrm{g} / \mathrm{L}) \\
\text { Cesium (total) ( } \mu \mathrm{g} / \mathrm{L})\end{array}$ & 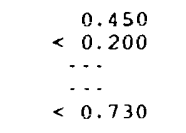 & B & 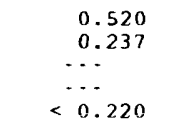 & $\begin{array}{l}\mathrm{B} \\
\mathrm{B}\end{array}$ & 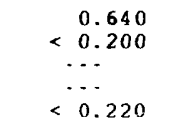 & B & $\begin{array}{l}<0.440 \\
<0.200 \\
\cdots \\
< \\
<0.220\end{array}$ & \\
\hline $\begin{array}{l}\text { Cesium (dissolved) ( } \mu \mathrm{g} / \mathrm{L}) \\
\text { Chromium (total) ( } \mu \mathrm{g} / \mathrm{L}) \\
\text { Chromium (dissolved) ( } \mu \mathrm{g} / \mathrm{L}) \\
\text { Cobalt (total) ( } \mathrm{gg} / \mathrm{L}) \\
\text { Cobalt (dissolved) ( } \mu \mathrm{g} / \mathrm{L})\end{array}$ & 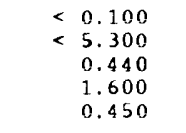 & $\begin{array}{l}\text { B } \\
\text { B } \\
\text { B }\end{array}$ & $\begin{array}{l}0.284 \\
2.500 \\
0.285 \\
1.200 \\
0.597\end{array}$ & $\begin{array}{l}\text { B } \\
\text { B } \\
B \\
B \\
B\end{array}$ & $\begin{array}{l}0.832 \\
5.300 \\
0.422 \\
1.300 \\
0.203\end{array}$ & $\begin{array}{l}\mathrm{B} \\
\mathrm{B} \\
\mathrm{B} \\
\mathrm{B} \\
\mathrm{B}\end{array}$ & $\begin{array}{l}<0.100 \\
<1.200 \\
<0.200 \\
<0.220 \\
<0.100\end{array}$ & \\
\hline 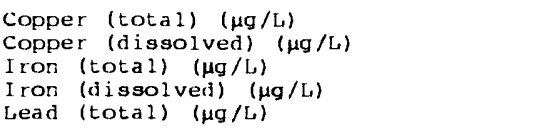 & $\begin{array}{r}8.000 \\
4.000 \\
4.040 .000 \\
33.860 \\
7.200\end{array}$ & $\begin{array}{l}\text { B } \\
\text { B }\end{array}$ & $\begin{array}{r}7.600 \\
2.659 \\
2.330 .000 \\
28.780 \\
3.400\end{array}$ & $\begin{array}{l}\mathrm{B} \\
\mathrm{B} \\
\mathrm{B}\end{array}$ & $\begin{array}{r}7.900 \\
3.363 \\
3,390.000 \\
49.130 \\
5.900\end{array}$ & $\begin{array}{l}\mathrm{B} \\
\mathrm{B} \\
\mathrm{B}\end{array}$ & $\begin{array}{r}<2.200 \\
<1.000 \\
102.000 \\
<\quad 10.000 \\
<\quad 0.220\end{array}$ & B \\
\hline $\begin{array}{l}\text { Lead (dissolved) ( } \mu \mathrm{g} / \mathrm{L}) \\
\text { Lithium (total) ( } \mathrm{gg} / \mathrm{L}) \\
\text { Lithium (dissolved) ( } \mu \mathrm{g} / \mathrm{L}) \\
\text { Manganese (total) ( } \mathrm{g} / \mathrm{L}) \\
\text { Manganese (rissolved) ( }(\mu \mathrm{g} / \mathrm{L})\end{array}$ & $\begin{array}{r}0.150 \\
4.200 \\
4.550 \\
113.000 \\
21.420\end{array}$ & $\begin{array}{l}\mathrm{B} \\
\mathrm{B} \\
\mathrm{B}\end{array}$ & $\begin{array}{r}<0.300 \\
<6.500 \\
<5.906 \\
213.000 \\
46.786\end{array}$ & & $\begin{array}{r}0.250 \\
<\quad 2.200 \\
<\quad 1.233 \\
231.000 \\
12.386\end{array}$ & B & $\begin{array}{r}<0.100 \\
<\quad 2.200 \\
<\quad 1.000 \\
29.900 \\
9.270\end{array}$ & $\begin{array}{l}\mathrm{B} \\
\mathrm{B}\end{array}$ \\
\hline $\begin{array}{l}\text { Mercury (tota } 1)(\mu g / L) \\
\text { Mercury (dissolved) ( } \mu \mathrm{g} / \mathrm{L}) \\
\text { Molybdenum (total) ( } \mathrm{gg} / \mathrm{L}) \\
\text { Molybdenum (dissolved) ( } \mu \mathrm{g} / \mathrm{L}) \\
\text { Nickel (total) ( } \mathrm{gg} / \mathrm{L})\end{array}$ & $\begin{array}{r}<0.200 \\
<0.200 \\
<\quad 7.100 \\
8.180 \\
5.500\end{array}$ & $\begin{array}{l}\mathrm{B} \\
\mathrm{B}\end{array}$ & $\begin{array}{r}<0.200 \\
<0.200 \\
<\quad 1.800 \\
1.973 \\
\\
2.000\end{array}$ & $\begin{array}{l}\mathrm{B} \\
\mathrm{B}\end{array}$ & $\begin{aligned}< & 0.200 \\
< & 0.200 \\
< & 0.530 \\
< & 0.596 \\
& 1.500\end{aligned}$ & B & $\begin{array}{l}<0.200 \\
<0.200 \\
<0.220 \\
<0.710 \\
<1.300\end{array}$ & \\
\hline $\begin{array}{l}\text { Nlckel (dissolved) }(\mu \mathrm{g} / \mathrm{L}) \\
\text { Selenium (total) ( } \mu \mathrm{g} / \mathrm{L}) \\
\text { Selenium (dissolved) ( } \mu \mathrm{g} / \mathrm{L}) \\
\text { silicon (total) } \\
\text { Silicon (dissolved) ( } \mu \mathrm{g} / \mathrm{L})\end{array}$ & $\begin{array}{r}2.810 \\
<6.700 \\
<3.000 \\
11,300.000 \\
2.597 .180\end{array}$ & B & $\begin{array}{r}3.889 \\
9.600 \\
8.567 \\
9.920 .000 \\
4.290 .000\end{array}$ & $\begin{array}{l}\mathrm{B} \\
\mathrm{B}\end{array}$ & $\begin{array}{r}1.278 \\
<\quad 6.700 \\
<3.000 \\
14,000.000 \\
1.080 .000\end{array}$ & B & $\begin{array}{r}<0.600 \\
<6.700 \\
<3.000 \\
<111.000 \\
<100.000\end{array}$ & \\
\hline $\begin{array}{l}\text { Silve: (total) ( } \mu \mathrm{g} / \mathrm{L}) \\
\text { Silver (dissolved) ( } \mu \mathrm{g} / \mathrm{L}) \\
\text { Strontium (total) ( } \mathrm{g} / \mathrm{L}) \\
\text { Strontium (dissolved) ( } \mu \mathrm{g} / \mathrm{L}) \\
\text { Thallium (total) ( } \mathrm{gg} / \mathrm{L})\end{array}$ & $\begin{array}{l}<0.220 \\
<0.100 \\
142.000 \\
143.050 \\
<0.220\end{array}$ & & $\begin{array}{l}<0.220 \\
<0.100 \\
361.000 \\
351.350 \\
<0.220\end{array}$ & & $\begin{array}{r}<0.220 \\
<\quad 0.100 \\
68.000 \\
62.009 \\
<\quad 0.220\end{array}$ & & $\begin{aligned}< & 0.220 \\
< & 0.100 \\
& 2.200 \\
& 1.450 \\
< & 0.220\end{aligned}$ & B \\
\hline
\end{tabular}


Table 42.-- Water-quality data, water year 1995--continued

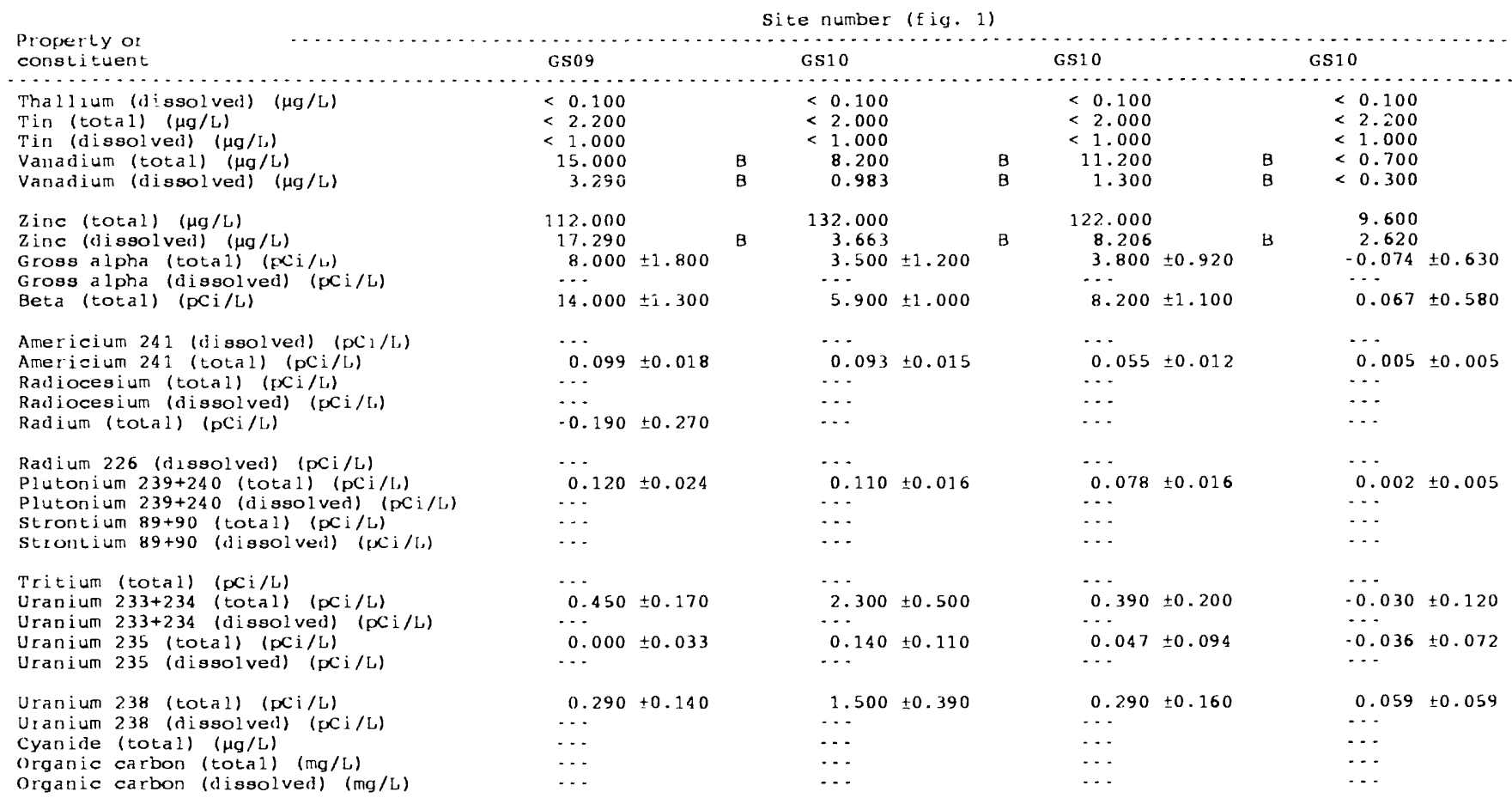


Table 42.-- Water-quality data, water year 1995--continued

\begin{tabular}{|c|c|c|c|c|c|c|}
\hline $\begin{array}{l}\text { Property or } \\
\text { constituent }\end{array}$ & $\begin{array}{c}\text { GS } 11 \\
\ldots . . .\end{array}$ & $\begin{array}{c}\text { Site number } \\
\text { GS16 }\end{array}$ & $\begin{array}{r}11 \\
\text { GS17 }\end{array}$ & & Sw0 27 & \\
\hline $\begin{array}{l}\text { Beginning date (mmddyy) } \\
\text { Beginning time (hhmm) } \\
\text { Ending date (mmddyy) } \\
\text { Ending time (hhmm) } \\
\text { Mean discharge (cfs) }\end{array}$ & $\begin{array}{l}10 / 25 / 94 \\
0858 \\
11 / 02 / 94 \\
0058 \\
1.420\end{array}$ & $\begin{array}{c}10 / 17 / 94 \\
1327 \\
10 / 17 / 94 \\
1452 \\
0.260\end{array}$ & $\begin{array}{l}11 / 05 / 94 \\
1424 \\
11 / 05 / 94 \\
1544 \\
0.410\end{array}$ & & $\begin{array}{l}10 / 17 / 94 \\
2228 \\
10 / 17 / 94 \\
2314 \\
0.490\end{array}$ & \\
\hline 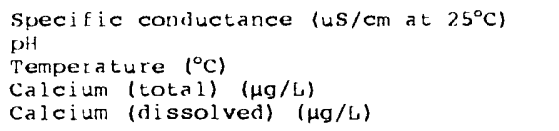 & $\begin{array}{r}550.000 \\
8.600 \\
6.600 \\
44,300.000 \\
43,117.871\end{array}$ & $\begin{array}{c}490.000 \\
7.500 \\
\cdots \\
72,800.000 \\
73,082.430\end{array}$ & $\begin{array}{r}570.000 \\
7.800 \\
5.400 \\
75,500.000 \\
74,859.563\end{array}$ & & $\begin{array}{c}490.000 \\
6.400 \\
\cdots \\
57,700.000 \\
60.042 .270\end{array}$ & \\
\hline $\begin{array}{l}\text { Magnesium (total) }\langle\mu g / L) \\
\text { Magnesium (dissolved) ( } \mu g / L) \\
\text { Sodium (total) ( } \mu g / L) \\
\text { Sodium (dissolved) ( } \mu g / L) \\
\text { Potassium (total) ( } \mu g / L\rangle\end{array}$ & $\begin{array}{r}11,200.000 \\
11,255.130 \\
51,900.000 \\
53,059.961 \\
9,210.000\end{array}$ & $\begin{array}{r}9,550.000 \\
9,191.830 \\
15,400.000 \\
18,136.650 \\
5.730 .000\end{array}$ & $\begin{array}{r}12,700.000 \\
13,214.220 \\
26,300.000 \\
28.430 .381 \\
2,790.000\end{array}$ & B & $\begin{array}{r}9,730.000 \\
10.282 .330 \\
27,200.000 \\
31.446 .131 \\
7.170 .000\end{array}$ & \\
\hline $\begin{array}{l}\text { Potassium (dissolved) }(\mu \mathrm{g} / \mathrm{L}) \\
\text { Bicarbonate as calcium carbonate (mg/L) } \\
\text { Carbonate as calcium carbonate (mg/L) } \\
\text { Sulfate (total) (mg/L) } \\
\text { Chloride (total) (mg/L) }\end{array}$ & $\begin{array}{r}9,220.230 \\
113.000 \\
<\quad 10.000 \\
49.500 \\
68.800\end{array}$ & $\begin{array}{r}5,310.870 \\
150.000 \\
<\quad 10.000 \\
32.600 \\
52.200\end{array}$ & $\begin{array}{r}3,114.390 \\
150.000 \\
<\quad 10.000 \\
84.400 \\
40.800\end{array}$ & B & $\begin{array}{r}8,797.690 \\
119.000 \\
<\quad 10.000 \\
28.200 \\
44.600\end{array}$ & \\
\hline $\begin{array}{l}\text { Fluoride (total) (mg/L) } \\
\text { Suspended solids (mg/L) } \\
\text { Nitrate/nitrite (total) (mg/L) } \\
\text { Nitrate/nitrite (total (mg/L) } \\
\text { Phosphorus (total) (mg/L) }\end{array}$ & $\begin{array}{l}0.500 \\
16.000 \\
\cdots \\
\cdots\end{array}$ & $\begin{array}{l}0.370 \\
220.000 \\
\cdots \\
0.250 \\
\cdots\end{array}$ & $\begin{array}{l}0.430 \\
15.000 \\
\cdots \\
\cdots\end{array}$ & & $\begin{array}{l}0.490 \\
6.000 \\
\cdots \\
2.200 \\
\cdots\end{array}$ & \\
\hline $\begin{array}{l}\text { Aluminum (total) }(\mu g / L) \\
\text { Aluminum (dissolved) }(\mu g / L) \\
\text { Antimony (total) ( } \mu g / L) \\
\text { Antimony (dissolved) }(\mu g / L) \\
\text { Arsenic (total) }(\mu g / L)\end{array}$ & $\begin{array}{r}334.000 \\
<\quad 30.000 \\
<\quad 0.900 \\
3.910 \\
9.100\end{array}$ & $\begin{array}{r}8.560 .000 \\
<\quad 30.000 \\
<0.440 \\
<\quad 1.817 \\
2.300\end{array}$ & $\begin{array}{r}198.000 \\
<\quad 30.000 \\
<\quad 0.440 \\
3.630 \\
<\quad 2.200\end{array}$ & B & $\begin{array}{r}400.000 \\
<\quad 30.000 \\
<\quad 0.440 \\
<4.294 \\
<\quad 2.200\end{array}$ & \\
\hline 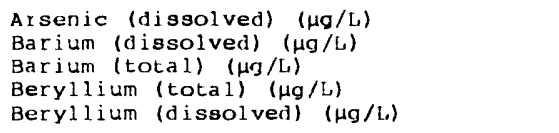 & $\begin{array}{r}8.240 \\
37.660 \\
47.000 \\
<\quad 0.220 \\
<\quad 0.100\end{array}$ & $\begin{array}{l}<1.000 \\
118.130 \\
199.000 \\
<0.220 \\
<0.100\end{array}$ & $\begin{array}{r}1.040 \\
109.660 \\
114.000 \\
<\quad 0.220 \\
<0.100\end{array}$ & $\begin{array}{l}\mathrm{B} \\
\mathrm{B} \\
\mathrm{B}\end{array}$ & $\begin{array}{r}1.683 \\
106.160 \\
111.000 \\
<0.220 \\
<0.100\end{array}$ & $\begin{array}{l}\mathrm{B} \\
\mathrm{B} \\
\mathrm{B}\end{array}$ \\
\hline 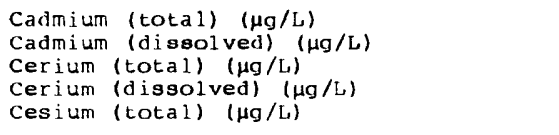 & $\begin{array}{l}<0.440 \\
<0.200 \\
\cdots \\
\quad \cdots \\
<0.220\end{array}$ & $\begin{array}{l}<0.440 \\
<0.200 \\
\cdots \\
< \\
<0.220\end{array}$ & $\begin{array}{l}<0.440 \\
<0.200 \\
\cdots \\
< \\
<0.220\end{array}$ & & $\begin{array}{l}<0.440 \\
<0.200 \\
\cdots \\
- \\
<0.220\end{array}$ & \\
\hline 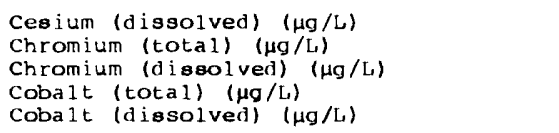 & $\begin{aligned}< & 0.100 \\
< & 0.600 \\
< & 0.200 \\
& 1.900 \\
& 1.760\end{aligned}$ & $\begin{array}{r}0.100 \\
5.700 \\
< \\
0.200 \\
2.600 \\
0.533\end{array}$ & $\begin{aligned}< & 0.100 \\
< & 0.440 \\
< & 0.200 \\
& 0.820 \\
& 0.620\end{aligned}$ & $\begin{array}{l}\mathrm{B} \\
\mathrm{B}\end{array}$ & $\begin{aligned} & 0.374 \\
< & 0.440 \\
< & 0.200 \\
& 0.530 \\
& 0.630\end{aligned}$ & $\begin{array}{l}\mathrm{B} \\
\mathrm{B}\end{array}$ \\
\hline 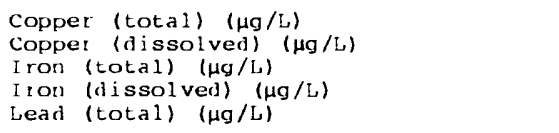 & $\begin{array}{r}2.300 \\
1.860 \\
260.000 \\
<\quad 10.000 \\
0.520\end{array}$ & $\begin{array}{r}<6.500 \\
<1.000 \\
6,670.000 \\
25.670 \\
9.100\end{array}$ & $\begin{array}{r}3.000 \\
<\quad 1.000 \\
279.000 \\
23.480 \\
0.350\end{array}$ & $\begin{array}{l}\mathrm{B} \\
\mathrm{B}\end{array}$ & $\begin{array}{r}4.200 \\
2.574 \\
246.000 \\
13.000 \\
15.800\end{array}$ & B \\
\hline 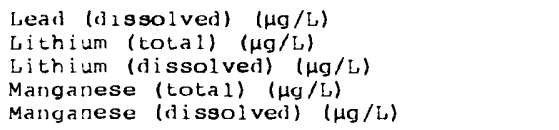 & $\begin{array}{r}<.100 \\
8.800 \\
10.990 \\
112.000 \\
2.130\end{array}$ & $\begin{array}{r}<0.300 \\
<7.300 \\
<3.240 \\
217.000 \\
4.888\end{array}$ & $\begin{array}{r}0.100 \\
5.000 \\
6.510 \\
19.400 \\
2.870\end{array}$ & $\begin{array}{l}\mathrm{B} \\
\mathrm{B} \\
\mathrm{B} \\
\mathrm{B}\end{array}$ & $\begin{array}{r}0.100 \\
<\quad 6.700 \\
<\quad 6.896 \\
22.700 \\
9.857\end{array}$ & $\begin{array}{l}\text { B } \\
\text { B }\end{array}$ \\
\hline 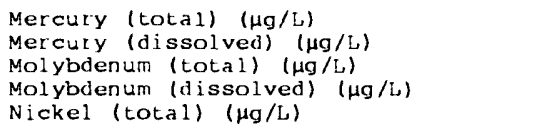 & $<\begin{array}{r}0.200 \\
<\quad 0.200 \\
8.800 \\
9.850 \\
5.300\end{array}$ & 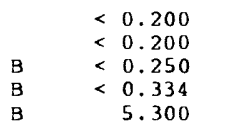 & $\begin{aligned}< & 0.200 \\
< & 0.200 \\
< & 0.270 \\
< & 1.250 \\
& 4.000\end{aligned}$ & B & $\begin{aligned}< & 0.200 \\
< & 0.200 \\
< & 1.700 \\
& 1.954 \\
& 6.200\end{aligned}$ & $\begin{array}{l}\text { B } \\
\text { B }\end{array}$ \\
\hline 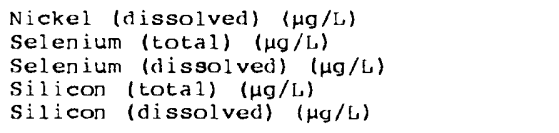 & $\begin{array}{r}4.820 \\
17.000 \\
7.260 \\
5.040 .000 \\
4.425 .720\end{array}$ & $\begin{array}{r}3.345 \\
<6.700 \\
<3.000 \\
35,700.000 \\
12.900 .000\end{array}$ & $\begin{array}{r}3.380 \\
6.700 \\
4.060 \\
10.500 .000 \\
10.310 .960\end{array}$ & B & $\begin{array}{r}4.310 \\
<6.700 \\
3.581 \\
5.850 .000 \\
5,050.000\end{array}$ & B \\
\hline 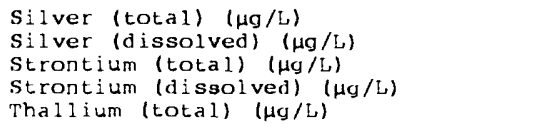 & $\begin{array}{l}<0.220 \\
<0.100 \\
253.000 \\
259.260 \\
<0.220\end{array}$ & $\begin{array}{l}<0.220 \\
<0.100 \\
258.000 \\
240.140 \\
<0.220\end{array}$ & $\begin{array}{l}<0.220 \\
<0.100 \\
336.000 \\
345.250 \\
<\quad 0.220\end{array}$ & & $\begin{array}{l}<0.220 \\
<0.100 \\
277.000 \\
285.930 \\
<0.220\end{array}$ & \\
\hline
\end{tabular}


Table 42.- Water-quality data, water year 1995--continued

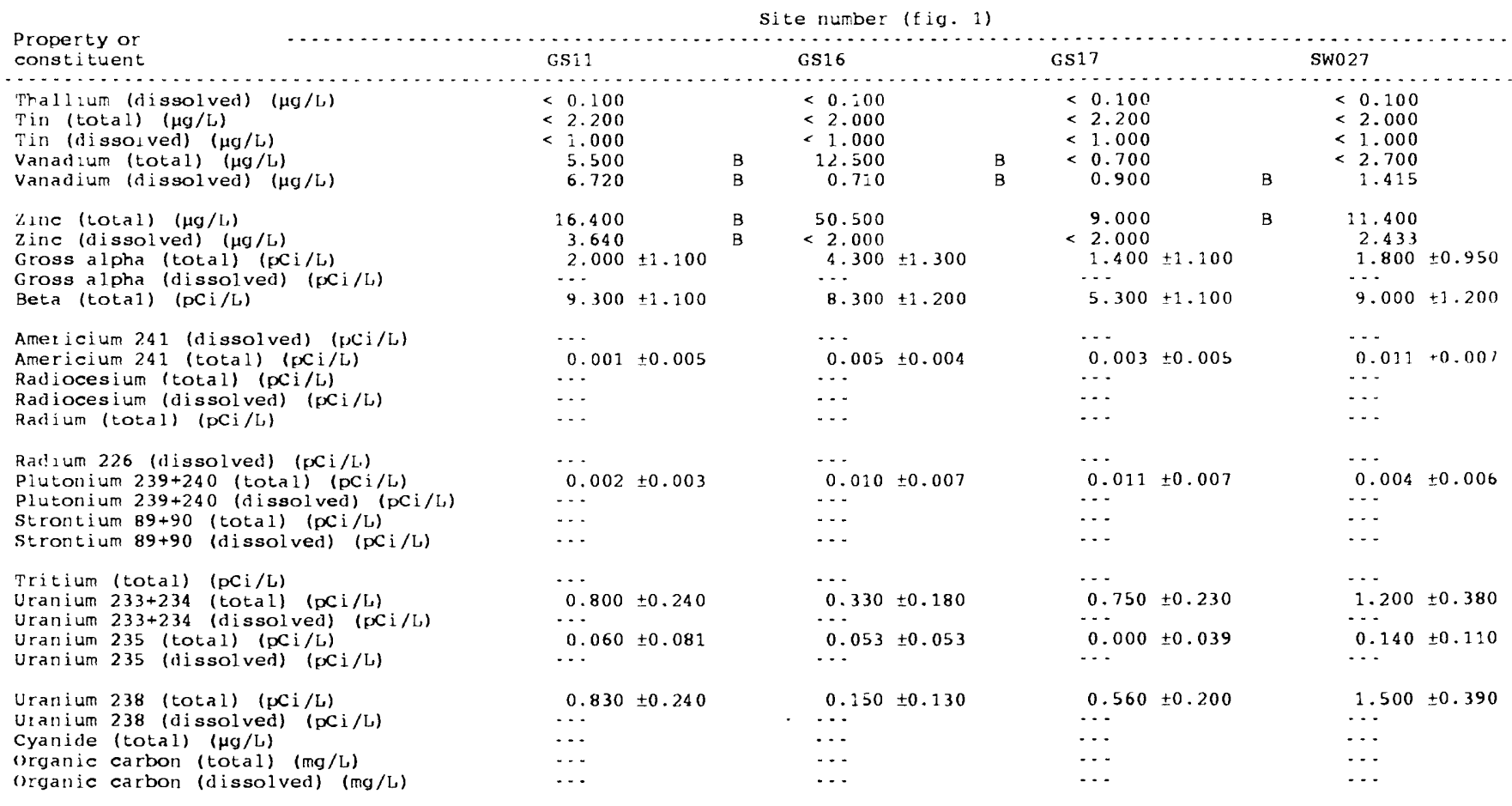


Table 42.-- Water-quality data, water year 1995--continued

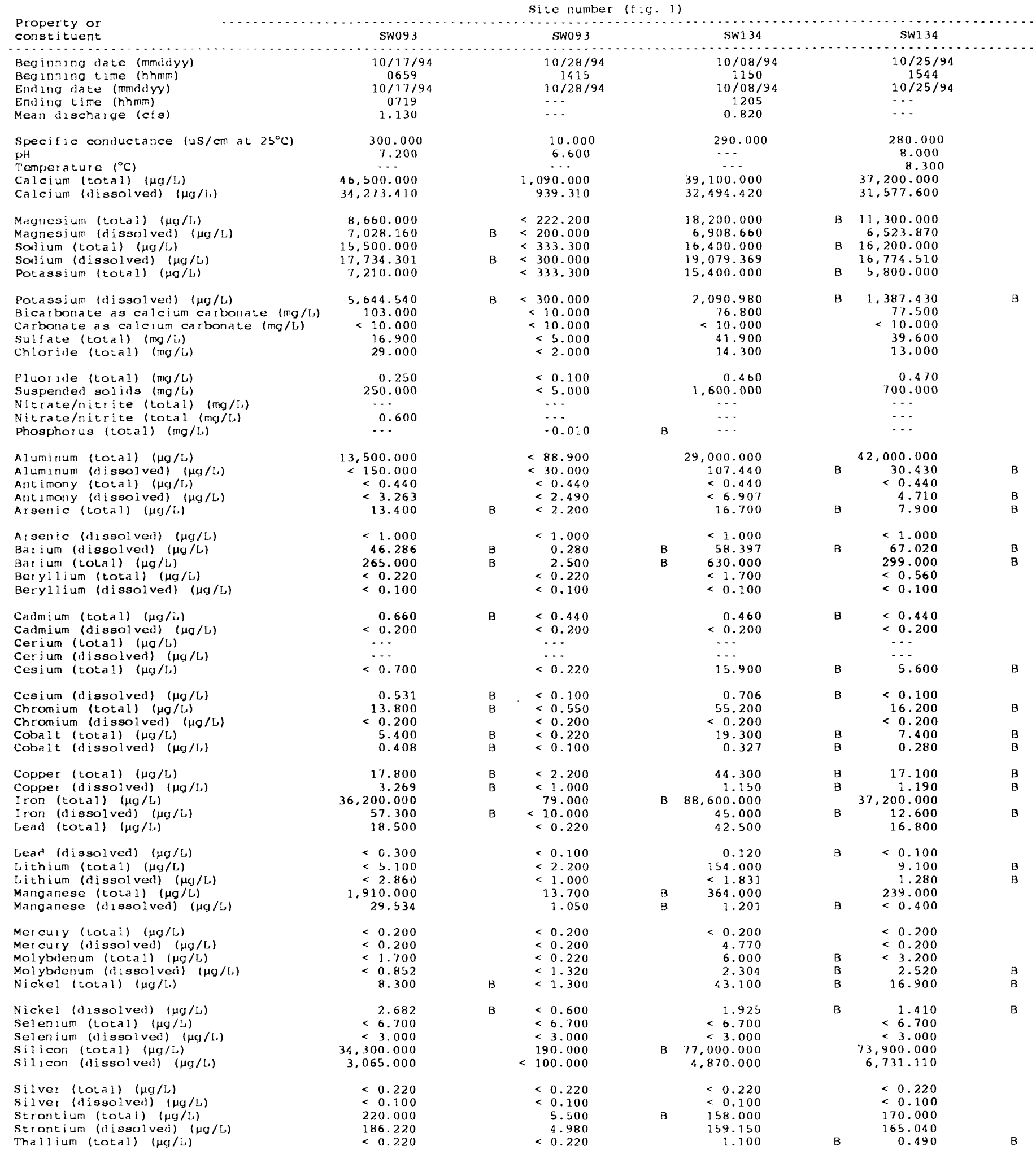


Table 42.-- Water-quality data, water year 1995--continued

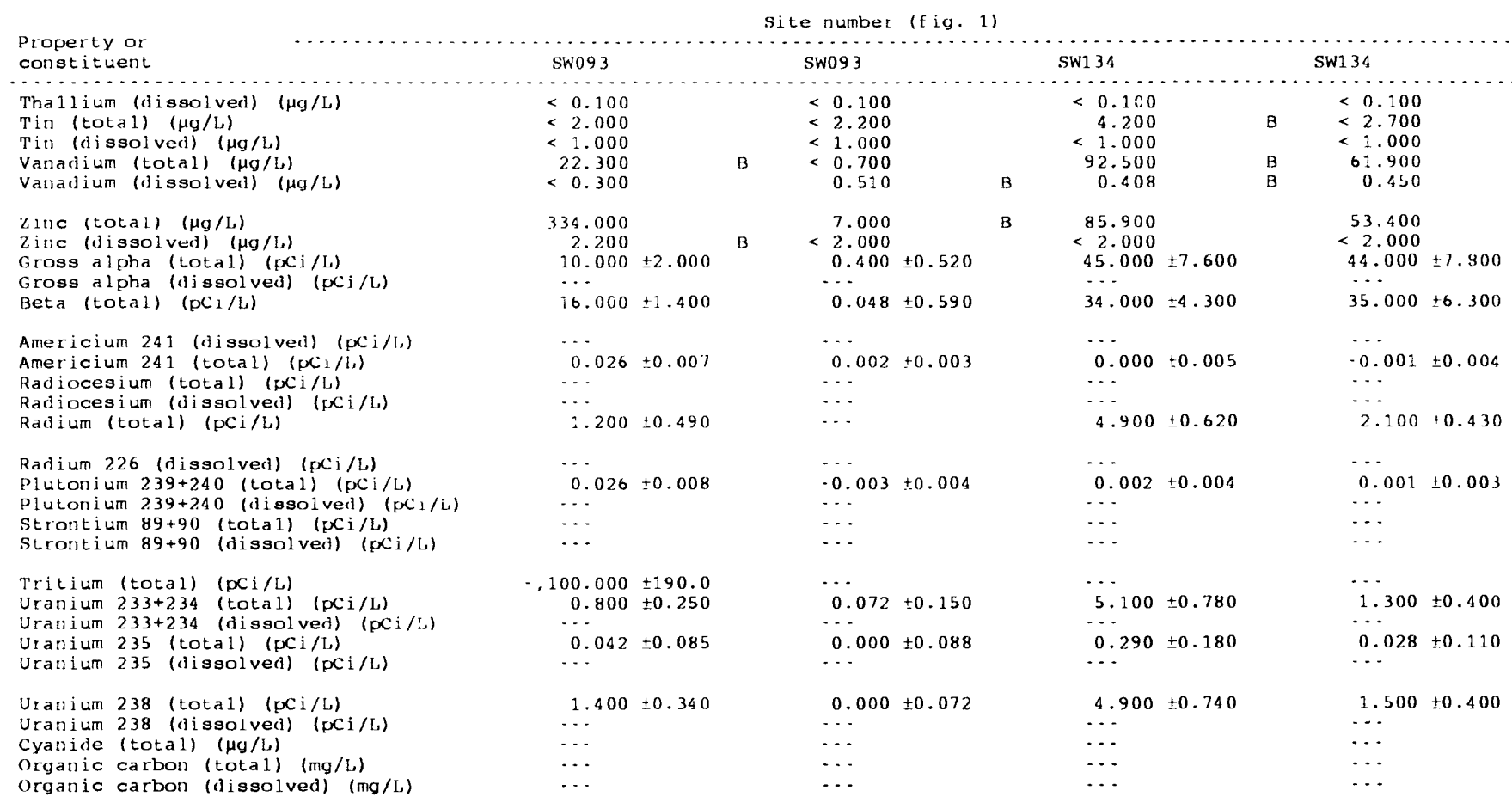


Table 42.-- Water-quality data, water year 1995--continued

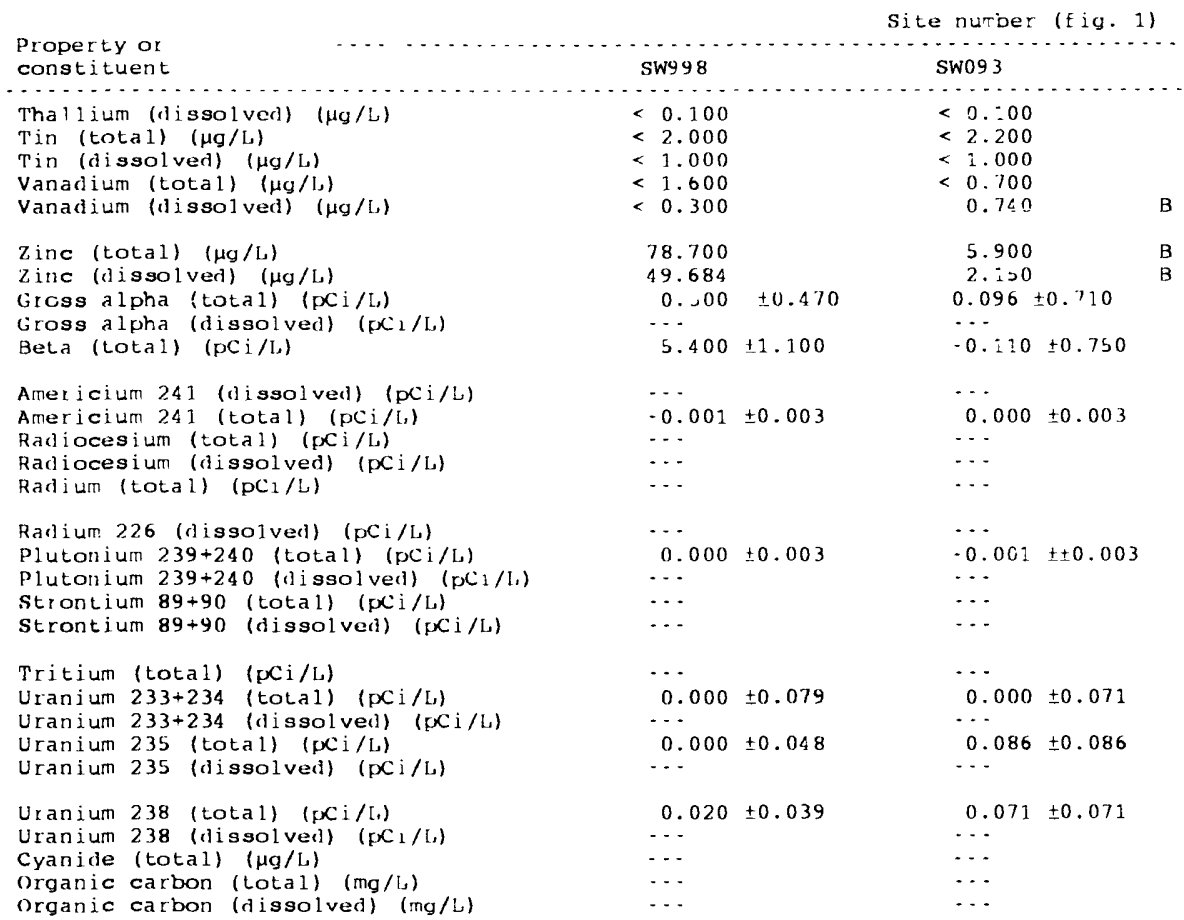


Table 43. Suspended-sediment data, water year 1994

[Time noted is start time for automatic (composite) samples, and instantaneous time for manual samples; discharge noted is mean discharge for automatic (composite) samples, and instantaneous discharge for manual samples.]

\begin{tabular}{|c|c|c|c|c|c|c|c|c|}
\hline $\begin{array}{l}\text { USGS station } \\
\text { identification }\end{array}$ & $\begin{array}{c}\text { Site } \\
\text { number }\end{array}$ & $\begin{array}{c}\text { Date } \\
\text { (mmddyy) }\end{array}$ & $\begin{array}{c}\text { Time } \\
\text { (hhmm) }\end{array}$ & $\begin{array}{c}\text { Discharge } \\
\left(\mathrm{ft}^{3} / \mathrm{s}\right)\end{array}$ & $\begin{array}{c}\text { Concen- } \\
\text { tration } \\
\text { (mg/L) }\end{array}$ & $\begin{array}{l}\text { Silt } \\
\text { clay } \\
\text { (per- } \\
\text { cent) }\end{array}$ & $\begin{array}{l}\text { Sand } \\
\text { (per- } \\
\text { cent) }\end{array}$ & $\begin{array}{l}\text { Sampling } \\
\text { method }\end{array}$ \\
\hline \multirow[t]{3}{*}{395342105110800} & GSO9 & $09 / 21 / 94$ & 1248 & 0.90 & 68 & 86 & 14 & Manual \\
\hline & & $09 / 21 / 94$ & 1258 & 0.85 & 28 & 96 & 4 & Automatic \\
\hline & & $09 / 21 / 94$ & 1300 & 0.90 & 24 & 81 & 19 & Manual \\
\hline \multirow[t]{11}{*}{395335105112700} & GS10 & $05 / 28 / 94$ & 1219 & 3.25 & 1,250 & 98 & 2 & Automatic \\
\hline & & $05 / 31 / 94$ & 1759 & 5.62 & 1,650 & 95 & 5 & Automatic \\
\hline & & $06 / 20 / 94$ & 2031 & 0.22 & 199 & 97 & 3 & Automatic \\
\hline & & $06 / 21 / 94$ & 1838 & 4.33 & 1,610 & 96 & 4 & Automatic \\
\hline & & $06 / 22 / 94$ & 1810 & 0.81 & 268 & 97 & 3 & Automatic \\
\hline & & $08 / 31 / 94$ & 1642 & 0.44 & 101 & 93 & 7 & Automatic \\
\hline & & $09 / 21 / 94$ & 1226 & 0.68 & 189 & 99 & 1 & Automatic \\
\hline & & $09 / 21 / 94$ & 1233 & 1.25 & 522 & 98 & 2 & Manual \\
\hline & & $09 / 21 / 94$ & 1235 & 1.18 & 5,238 & 100 & 0 & Manual \\
\hline & & $09 / 21 / 94$ & 1237 & 1.15 & 324 & 99 & 1 & Manual \\
\hline & & $09 / 22 / 94$ & 1202 & 0.63 & 30 & 100 & 0 & Automatic \\
\hline \multirow[t]{2}{*}{395403105104700} & GSII & $06 / 20 / 94$ & 1024 & 1.67 & 7 & 77 & 23 & Automatic \\
\hline & & $07 / 23 / 94$ & 0639 & 1.63 & 8 & 43 & 57 & Automatic \\
\hline 395358105110500 & GSI2 & $09 / 29 / 94$ & 1254 & 1.50 & 58 & 100 & 0 & Automatic \\
\hline 395301105120800 & GSI6 & $09 / 22 / 94$ & 1240 & 0.12 & 267 & 83 & 17 & Automatic \\
\hline \multirow[t]{4}{*}{395349105114900} & SW093 & $05 / 28 / 94$ & 1324 & 1.29 & 1,510 & 96 & 4 & Automatic \\
\hline & & $05 / 31 / 94$ & 1913 & 1.81 & 1,090 & 95 & 5 & Automatic \\
\hline & & $06 / 21 / 94$ & 1841 & 3.23 & 1,140 & 92 & 8 & Automatic \\
\hline & & $06 / 22 / 94$ & 1804 & 0.55 & 126 & 96 & 4 & Automatic \\
\hline \multirow[t]{8}{*}{395332105124600} & SW998 & $05 / 28 / 94$ & 1335 & -- & 333 & 96 & 4 & Automatic \\
\hline & & $05 / 31 / 94$ & 1913 & 3.42 & 392 & 97 & 3 & Automatic \\
\hline & & $06 / 21 / 94$ & 1913 & 0.10 & 23 & 72 & 28 & Automatic \\
\hline & & $06 / 22 / 94$ & 1804 & 2.43 & 180 & 95 & 5 & Automatic \\
\hline & & $08 / 10 / 94$ & 2145 & 4.40 & 292 & 96 & 4 & Automatic \\
\hline & & $08 / 31 / 94$ & 1630 & 2.65 & 35 & 90 & 10 & Automatic \\
\hline & & $09 / 21 / 94$ & 1243 & 0.11 & 37 & 100 & 0 & Automatic \\
\hline & & $09 / 21 / 94$ & 1558 & 0.21 & 36 & 100 & 0 & Automatic \\
\hline
\end{tabular}


Table 44. Suspended-sediment data, water year 1995

[Time noted is start time for automatic (composite) samples. and instantaneous time for manual samples; discharge noted is mean discharge for automatic (composite) samples. and instantaneous discharge for manual samples.]

\begin{tabular}{|c|c|c|c|c|c|c|c|c|}
\hline $\begin{array}{l}\text { USGS station } \\
\text { identification }\end{array}$ & $\begin{array}{c}\text { Site } \\
\text { number }\end{array}$ & $\begin{array}{c}\text { Date } \\
\text { (mmddyy) }\end{array}$ & $\underset{\text { (hhmm) }}{\text { Time }}$ & $\begin{array}{c}\text { Discharge } \\
\left(\mathrm{ft}^{3} / \mathrm{s}\right)\end{array}$ & $\begin{array}{c}\text { Concen- } \\
\text { tration } \\
\text { (mg/L) }\end{array}$ & $\begin{array}{c}\text { Silt } \\
\text { clay } \\
\text { (percent) }\end{array}$ & $\begin{array}{c}\text { Sand } \\
\text { (percent) }\end{array}$ & $\begin{array}{l}\text { Sampling } \\
\text { method }\end{array}$ \\
\hline \multirow[t]{2}{*}{395240105095500} & GS01 & $04 / 27 / 95$ & 1325 & 3.60 & 21 & --- & $\cdots$ & Manual \\
\hline & & $04 / 27 / 95$ & 1402 & 3.65 & 38 & --- & --- & Manual \\
\hline 395253105095500 & GS02 & $11 / 15 / 94$ & 1210 & 0.01 & 3 & 50 & 50 & Automatic \\
\hline 395407 IDS095900 & GS03 & $04 / 27 / 95$ & 1430 & 5.18 & 73 & $\cdots$ & --- & Manual \\
\hline 395306105131700 & GS05 & $11 / 14 / 94$ & 1540 & 0.28 & 7 & 93 & 7 & Automatic \\
\hline \multirow[t]{3}{*}{395342105110800} & GS09 & $10 / 17 / 94$ & 1005 & 1.90 & 54 & 97 & 3 & Automatic \\
\hline & & $10 / 17 / 94$ & 1346 & 1.77 & 35 & 97 & 3 & Automatic \\
\hline & & $11 / 08 / 94$ & 1649 & 2.31 & 114 & 98 & 2 & Automatic \\
\hline \multirow[t]{3}{*}{395335105112700} & GS10 & $10 / 17 / 94$ & 0625 & 0.61 & 47 & 97 & 3 & Automatic \\
\hline & & $10 / 17 / 94$ & 0951 & 2.12 & 67 & 98 & 2 & Automatic \\
\hline & & $04 / 28 / 95$ & 1305 & 0.46 & 40 & -- & --- & Manual \\
\hline 395403105104700 & GSII & $10 / 25 / 94$ & 0858 & 1.42 & 17 & 99 & 1 & Automatic \\
\hline 395301105120800 & GS16 & $10 / 17 / 94$ & 1327 & 0.26 & 235 & 85 & 15 & Automatic \\
\hline 395309105114100 & GS17 & $11 / 05 / 94$ & 1424 & 0.41 & 10 & 98 & 2 & Automatic \\
\hline \multirow[t]{2}{*}{395313105110500} & SW027 & $10 / 17 / 94$ & 2228 & 0.48 & 7 & 90 & 10 & Automatic \\
\hline & & $04 / 28 / 95$ & 1305 & 0.69 & 12 & --- & --- & Manual \\
\hline \multirow[t]{2}{*}{395349105114900} & SW093 & $10 / 17 / 94$ & 0659 & 1.13 & 269 & 99 & 1 & Automatic \\
\hline & & $04 / 28 / 95$ & 1315 & 0.90 & 34 & $\cdots$ & --- & Manual \\
\hline \multirow[t]{2}{*}{395331105134400} & SW 134 & $10 / 08 / 94$ & 1150 & 0.82 & 1,760 & 100 & 0 & Automatic \\
\hline & & $10 / 25 / 94$ & 1544 & --- & 818 & 100 & 0 & Automatic \\
\hline \multirow[t]{2}{*}{395332105124600} & SW998 & $10 / 15 / 94$ & 1049 & 0.17 & 7 & 91 & 9 & Automatic \\
\hline & & $04 / 28 / 95$ & 1340 & 0.39 & 8 & 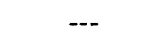 & -.. & Manual \\
\hline
\end{tabular}


APPENDIXES 


\section{Appendix 1: Discharge Data Collection and Computation}

The data obtained at a continuous surface-water gaging station on a stream or conveyance, such as an irrigation ditch, consist of a continuous record of stage, individual measurements of discharge throughout a range of stages, and notations regarding factors that might affect the relation of stage to discharge. These data, together with supplemental information such as climatologic records, are used to compute daily mean discharges.

Continuous records of stage are obtained with electronic recorders that store stage values at selected time intervals or with satellite data-collection platforms that transmit near real-time data at selected time intervals to office computers. Mcasurements of discharge are made with current meters, using methods adapted by the USGS as a result of experience accumulated since 1880 , or with flumes or weirs that are calibrated to provide a relation of observed stage to discharge. These methods are described by Carter and Davidian (1968) and by Rantz and others (1982).

In computing discharge records, results of individual measurements are plotted against the corresponding stages, and stage-discharge relation curves are constructed. From these curves, rating tables indicating the computed discharge for any stage within the range of the measurements are prepared. If it is necessary to define extremes of discharge outside the range of the current-meter measurements, the curves are extended using: (1) Logarithmic plotting; (2) velocity-area studies; (3) results of indirect measurements of peak discharge, such as slope-area or contracted-opening measurements, and computations of flow over dams or weirs; or (4) step-backwater techniques.

Daily mean discharges are computed by applying the daily mean stages (gage heights) to the stage-discharge curves or tables. If the stage-discharge relation is subject to change because of frequent or continual change in the physical features that form the control, the daily mean discharge is determined by the shifting-control method, in which correction factors based on the individual discharge measurements and notes of the personnel making the measurements are applied to the gage heights before the discharges are determined from the curves or tables. This shifting-control method also is used if the stage-discharge relation is changed temporarily because of aquatic vegetation growth or debris on the control. For some gaging stations, formation of ice in the winter can obscure the stage-discharge relations so that daily mean discharges need to be estimated from other information such as temperature and precipitation records, notes of observations, and records for other gaging stations in the same or nearby basins for comparable periods.

For most gaging stations there might be periods when no gage-height record is obtained or the recorded gage height is so faulty that it cannot be used to compute daily mean discharge or contents. This record loss occurs when recording instruments malfunction or otherwise fail to operate properly, intakes are plugged, the float is frozen in the stilling well, or for various other reasons. For such periods, the daily discharges are estimated from the recorded range in stage, previous or following record, discharge measurements, climatologic records, and comparison with other gaging-station records from the same or nearby basins. Information explaining how estimated daily-discharge values are identified in gaging-station records is provided in the "Identifying Estimated Daily Discharge" section of this Appendix.

\section{Data presentation}

The daily mean discharge tables published for each continuous-record surface-water gaging station consist of four parts: the station description; the table of daily mean discharge values for the water year with summary data; a tabular statistical summary of monthly mean discharge data for the water year; and a summary statistics table that includes statistical data of annual, daily, and instantaneous discharge, and summaries of 7-day low-flow minimums, annual runoff, and flow duration. 


\section{Station description}

The station description provides, under various headings, descriptive information including gaging-station location, drainage area, period of record, gage information, historical extremes outside the period of record, record accuracy, and other remarks pertinent to gaging-station operation and regulation. The following information is provided with each continuous record of daily mean discharge:

SITE NUMBER.--This entry identifies the unique site identification number.

STATION IDENTIFICATION.--This entry identifics the unique, 15-digit number assigned to a gaging station by the USGS for use in ADAPS. This number generally is the latitude and longitude of the gage with a sequence number $(00)$ at the end.

LOCATION.--This entry identifies the gaging-station latitude and longitude (given in degrees, minutes, and seconds); a landline location designation; the hydrologic unit number; county; and geographic location. Gagingstation latitudes, longitudes, and geographic locations were provided by EG\&G Rocky Flats, Incorporated (1993b).

DRAINAGE AREA.--This entry identifies the drainage area (in square miles) of the gaged basin. If, because of unusual natural conditions or anthropogenic controls, some part of the basin does not contribute flow to the total flow measured at the gage, the noncontributing drainage area also is identified. Drainage area usually is measured using digital techniques and the most accurate maps available. Because the type of map available might vary from one drainagc basin to another, the accuracy of digitized drainage areas also can vary. Drainage areas are updated as better maps become available. Some of the gaging stations included in this report measure stage and discharge in channels that convey water to or from reservoirs or other features; these channels might have little or no contributing drainage area. Drainage arcas in this report were provided by EG\&G Rocky Flats, Incorporated (1993b).

PERIOD OF RECORD.--This entry identifies the period for which there are published records at the gage. This entry includes the month and year of the start of publication of hydrologic records by the USGS and the words "to current year" if the records are to be continued into the following year. Periods for which hydrologic records are available, but not published by the USGS, might be noted.

GAGE.--This entry identifies: the type of gage currently in use; the datum of the current gage referred to sea level; and a condensed history of the types, locations, and datums of previous gages. Gage datum for each gaging station was provided by EG\&G Rocky Flats, Incorporated (1993b).

REMARKS.--This entry is used to provide information concerning the accuracy of the records, any special methods of computation, and conditions that affect natural flow at the gaging station. Other information might include average discharge data for the periud of record, extremes in data for the period of record and the current year, and any other unusual or noteworthy conditions.

\section{Daily Mean Discharge Values}

The daily mean discharge values computed for each gaging station during a water year are listed in the body of the data table. In the monthly summary part of the table, the line headed "TOTAL" lists the sum of the daily figures for each month; the line headed "MEAN" lists the average discharge, in cubic feet per second during the month; and the lines headed "MAX" and "MIN" list the maximum and minimum daily mean discharges for each month. Discharge for the month also is expressed in acre-feet (line headed "AC-FT"). Discharge, in cubic fect per second per square mile, and runoff, in inches or in acre-feet is omitted if there is extensive regulation or diversion or if the drainage area includes large noncontributing areas. In the yearly summary below the monthly summary, the appropriate discharges are listed for the calendar and water years. Missing records or periods prior to start of data collection are indicated by three dashes in place of the discharge. 


\section{Summary Statistics}

A section of the table titled SUMMARY STATISTICS follows the STATISTICS OF MONTHLY MEAN DATA section. This section consists of four columns, with the first column containing the statistics being reported. The section provides a statistical summary of annual and daily discharges, not only for the current water year but also for the previous calendar year and for a designated period, as appropriate. The designated period selected, WATER YEARS , consists of all of the gaging-station records in the specified water years, inclusive, including complete months of record for partial water years, if any, and usually coincides with the period of record for the gaging station. The water years for which the statistics are computed are consecutive, unless a break in the station record is indicated in the station description. All of the calculations for the statistical characteristics designated ANNUAL (these line headings are identified subsequently in this section), except for the ANNUAL SEVEN-DAY MINIMUM statistic, are for the designated period using complete water years.

The date or water year, as appropriate, of the first occurrence of each extreme discharge statistic is provided adjacent to the statistic. If the same extreme discharge occurs again, dates of repeated occurrence are noted in the REMARKS paragraph of the station description or in footnotes. Selected flow-duration-curve statistics and runoff data also are listed when a sufficient period of record is available. Runoff data are omitted if there is extensive regulation or diversion of flow in the drainage basin.

The following summary statistics data, as appropriate, are provided with each table of daily mean discharge. Summary statistics are not computed if no discharges occur during the water year. The following comments clarify information presented under the various line headings of the SUMMARY STATISTICS section of the table.

ANNUAL TOTAL.--The total of all daily mean discharges for a given water year.

ANNUAL MEAN.--The annual mean discharge for a given water year.

HIGHEST ANNUAL MEAN.--The maximum annual mean discharge occurring for the designated period. LOWEST ANNUAL MEAN.--The minimum annual mean discharge occurring for the designated period.

HIGHEST DAILY MEAN.--The maximum daily mean discharge for the year or for the designated period. LOWEST DAILY MEAN.--The minimum daily mean discharge for the year or for the designated period.

ANNUAL SEVEN-DAY MINIMUM.--The lowest mean discharge for 7 consecutive days for a calendar year or a water year. Most low-flow frequency analyses of annual 7-day minimum flows use a climatic year (April 1-March 31). The date shown in the "Summary Statistics" section of the table is the initial date of the 7-day period. (The 7-day minimum flow statistic is different than the 7-day 10-year low-flow statistic.)

ANNUAL RUNOFF (AC-FT).--Indicates the total quantity of water in runoff for a drainage area for the year, in acre-feet. One acre-foot is the quantity of water needed to cover I acre to a depth of $1 \mathrm{ft}$ and is equal to $43,560 \mathrm{ft}^{3}$ or about $326,000 \mathrm{gal}$.

10 PERCENT EXCEEDS.--The discharge that is exceeded by 10 percent of the flow for the designated period.

50 PERCENT EXCEEDS.--The discharge that is exceeded by 50 percent of the flow for the designated period.

90 PERCENT EXCEEDS.--The discharge that is exceeded by 90 percent of the flow for the designated period.

\section{Identifying Estimated Daily Discharge}

Estimated daily discharges published in the water-discharge tables of annual USGS water-data reports are identified by flagging individual daily values with the letter "e" and printing a table footnote, "e Estimated." 


\section{Accuracy of the Records}

The accuracy of discharge records depends primarily on: (1) The stability of the stage-discharge relation or, if the control is unstable, the frequency of discharge mcasurements; and (2) the accuracy of measurements of stage, measurements of discharge, and interpretation of records.

The accuracy attributed to the records is indicated under REMARKS. Excellent means that about 95 percent of the daily discharges are within 5 percent of their true value; good, within 10 percent; and fair, within 15 percent. Records that do not meet the criteria mentioned are rated poor. Different accuracies might be attributed to different parts of a given record.

Daily mean discharges in this report are listed to the nearest hundredth of a cubic foot per second for daily values less than $1 \mathrm{ft}^{3} / \mathrm{s}$ (a discharge of $0.01 \mathrm{ft}^{3} / \mathrm{s}$ is the smallest quantificd discharge); to the nearest tenth between 1.0 and $10 \mathrm{ft}^{3} / \mathrm{s}$; to whole numbers between 10 and $1,000 \mathrm{ft}^{3} / \mathrm{s}$; and to three significant figures for more than 1,000 $\mathrm{ft}^{3} / \mathrm{s}$.

\section{Other Records Available}

Information used in the preparation of the records in this report, such as discharge-measurement notes, gageheight records, temperature measurements, and rating tables, are on file with the USGS. Information on the availability of the unpublished information or on the published statistical analyses is available from USGS personnel involved with data collection at the Site. 


\section{Appendix 2: Glossary}

Terms related to surface-water flow, water-quality, and other hydrologic data are defined below. Except where noted, definitions are from Ugland and others (1995).

Acre-foot (AC-FT, acre-ft) is the quantity of water required to cover 1 acre to a depth of $1 \mathrm{ft}$ and is equal to $43,560 \mathrm{ft}^{3}$ or about 326,000 gal.

Cfs-day is the volume of water represented by flow of $1 \mathrm{ft}^{3} / \mathrm{s}$ for 24 hours. It is equivalent to $86,400 \mathrm{ft}^{3}$, about 1.9835 acre-ft, or about 646,000 gal.

Control designates a feature downstream from the gaging station that determines the stage-discharge relation at that gaging station. This feature might be a natural constriction of the channel, an artificial structure such as a weir, or a uniform cross section over a long reach of the channel.

Control structure is a structure on a stream or canal that is used to regulate the flow or stage of the stream.

Cubic foot per second $\left(\mathrm{ft}^{3} / \mathrm{s}\right)$ is the rate of discharge representing a volume of $1 \mathrm{ft}^{3}$ passing a given point during 1 second and is equivalent to $7.48 \mathrm{gal} / \mathrm{s}$ or $448.8 \mathrm{gal} / \mathrm{min}$.

Discharge is the volume of water (or more broadly, volume of water plus suspended sediment) that passes a given point within a given period of time.

Instantaneous discharge is the discharge at a particular instant of time.

Mean discharge (MEAN) is the arithmetic mean of individual daily mean discharges during a specific time. Dissolved refers to that material in a representative water sample that passes through a $0.45-\mu \mathrm{m}$ membrane filter. This definition is a convenient operational definition used by Federal agencies that collect water data. Determinations of dissolved constituents are made on subsamples of the filtrate.

Dissolved-solids concentration of water is determined either analytically by the residue-on-evaporation method or mathematically by totaling the concentrations of individual constituents reported in a comprehensive chemical analysis. During the analytical determination of dissolved solids, the bicarbonate (generally a major dissolved component of water) is converted to carbonate. Therefore, in the mathematical calculation of dissolved-solids concentration, the bicarbonate value, in milligrams per liter, is multiplied by 0.492 to indicate the change.

Drainage area of a stream at a specified location is that area, measured in a horizontal plane, enclosed by a topographic divide from which direct surface runoff from precipitation normally drains by gravity into the stream upstream from the specified point. Drainage areas in this report include all closed basins, or noncontributing areas, in the area unless otherwise noted.

Drainage basin is a part of the surface of the Earth that is occupied by a drainage system, which consists of a surface stream or body of impounded surface water and all tributary surface streams and bodies of impounded surface water.

Gage height is the water-surface elevation referred to some arbitrary gage datum. Gage height often is used interchangeably with the more general term "stage," although gage height is more appropriate when used with a reading on a gage.

Gaging station is a particular site on a stream, canal, lake, or reservoir where systematic observations of hydrologic data are obtained.

Hydrologic unit is a geographic area representing part or all of a surface drainage basin or distinct hydrologic feature as delineated by hydrologic unit maps of the State of Colorado (U.S. Geological Survey, 1976); each hydrologic unit is identified by an eight-digit number.

Micrograms per liter $(\mu \mathrm{g} / \mathrm{L})$ is a unit expressing the concentration of chemical constituents in solution as mass (micrograms) of solute per unit volume (liter) of water. One-thousand micrograms per liter is equivalent to $1 \mathrm{mg} / \mathrm{L}$.

Milligrams per liter $(\mathrm{MG} / \mathrm{L}, \mathrm{mg} / \mathrm{L})$ is a unit for expressing the concentration of chemical constituents in solution. Milligrams per liter represents the mass of solute per unit volume (liter) of water. Concentration of suspended sediment also is expressed in milligrams per liter and is based on the mass of dry sediment per liter of watersediment mixture. 
Partial-record station is a particular site where limited flow and/or water-quality data are collected systematically over a period of years for use in hydrologic analyses.

Scdiment is solid matcrial that originates mostly from disintegrated rocks and is transported by, suspended in, or deposited from water; it includes chemical and biochemical precipitates and decomposed organic material such as humus. The quantity, characteristics, and cause of sediment in streams are affected by environmental factors. Some major factors are degree of slope, length of slope, soii characteristics, land usage, and quantity and intensity of precipitation.

Bedload (tons) is the sediment that is transported in a stream by rolling, sliding, or skipping along the bed and very close to it.

Bedload discharge (tons/day) is the quantity of bedload measured by dry weight that moves past a section as bedload in a given time.

Suspended sediment is the sediment that at any given time is maintained in suspension by the upward components of turbulent currents or that exists in suspension as a colloid.

Suspended-sediment concentration is the velocity-weighted concentration of suspended sediment in the sampled zone (from the water surface to a point about $0.3 \mathrm{ft}$ above the bed) expressed as milligrams of dry sediment per liter of water-sediment mixture (milligrams per liter).

Mean concentration is the time-weighted concentration of suspended sediment passing a stream section during 24 hours.

Suspended-sediment discharge (tons/d) is the rate at which dry mass of sediment passes a section of a stream or is the quantity of sediment, as measured by dry mass or volume, that passes a section in a given time. It is calculated in units of tons per day as follows: concentration (milligrams per liter) times discharge (cubic feet per second) times 0.0027 .

Suspended-sediment load (tons) is a general term that refers to material in suspension. It is not synonymous with discharge or concentration.

Suspended-sediment sand break is the percent by weight of a suspended-sediment sample that is greater than or equal to $0.0625 \mathrm{~mm}$ in diameter (sand). The percent of the sample remaining is less than $0.0625 \mathrm{~mm}$ in diameter (silt/clay). (Daniel J. Gooding, U.S. Geological Survey, Cascades Volcano Observatory Sediment Laboratory, written commun., 1995)

Total-sediment discharge (tons/d) is the sum of the suspended-sediment discharge and the bedload discharge. It is the total quantity of sediment, as measured by dry mass or volume, that passes a section during a given time.

Total-sediment load, or total load, is a term that refers to the total mass of sediment (bedload plus suspended-sediment load) in transport. It is not synonymous with total-sediment discharge.

7-day 10-year low flow (7 Q10) is the discharge at the 10-year recurrence interval taken from a frequency curve of annual values of the lowest mean discharge for 7 consecutive days (the 7-day low flow).

Solute is any substance that is dissolved in water.

Specific conductance is a measure of the ability of a water to conduct an electrical current. It is expressed in microsiemens per centimeter at $25^{\circ} \mathrm{C}$. Specific conductance is related to the type and concentration of ions in solution and can be used for approximating the dissolved-solids content of the water. Empirical analysis of the relation between dissolved-solids concentration (in milligrams per liter) and specific conductance (in microsiemens per centimeter at $25^{\circ} \mathrm{C}$ ) indicates that the magnitude of dissolved-solids concentration commonly is between 55 and 75 percent of the magnitude of specific conductance (Hem, 1985, p. 67). This relation is not constant from stream to stream, and it can vary in the same source with changes in the composition of the water.

Stage is a general term for the height of the water surface of a stream or lake above an established datum plane.

Stage often is used interchangeably with the term "gage height" (Rantz and others, 1982).

Stage-discharge relation is the relation between gage height (stage) and the volume of water, per unit of time, flowing in a channel.

Streamflow is the discharge that occurs in a natural channel. Although the term "discharge" can be applied to the flow of a canal, the word "streamflow" uniquely describes the discharge in a surface stream course. The term 
"streamflow" is more gencral than "runoff" because streamflow might be applied to discharge whether or not it is affected by diversion or regulation.

Suspended (as used in tables of chemical analyses) refers to the amount (concentration) of undissolved material in a water/sediment mixture. It is associated with the material retained on a $0.45-\mu \mathrm{m}$ filter.

Tons per acre-foot indicates the dry mass of dissolved solids in 1 acre-ft of water. It is computed by multiplying the concentration of the constituent, in milligrams per liter, by 0.00136 .

Tons per day (T/DAY, tons/d) is the quantity of a substance in solution or suspension that passes a stream section during 24 hours.

Total is the total amount of a given constituent in a representative water/suspended-sediment sample, regardless of the physical or chemical form of the constituent. This term is used only when the analytical procedure assures measurement of at least 95 percent of the constituent present in the dissolved and in suspended phases of the sample. A knowledge of the expected form of the constituent in the sample, and of the analytical method used, is needed to judge when the results can be reported as total. (The word "total" does double duty here, indicating that the sample consists of a water/suspended-sediment mixture and that the analytical method determined all of the constituent in the sample.)

Total discharge is the total quantity of any individual constituent, as measured by dry mass or volume, that passes through a stream cross section per unit of time. This term needs to be qualified, such as total sediment discharge, total chloride discharge, and so on.

Weighted average indicates the discharge-weighted average. It is computed by multiplying the discharge for a sampling period by the concentrations of individual constituents for the corresponding period and dividing the sum of the products by the sum of the discharges. A discharge-weighted average approximates the composition of water that would be in a reservoir containing all the water passing a given location during the water year after thorough mixing in the reservoir. 